\title{
Myelin and networks
}

Citation for published version (APA):

Drenthen, G. S. (2020). Myelin and networks: Magnetic resonance imaging in epilepsy. [Doctoral Thesis, Maastricht University]. Gildeprint en Universitaire Pers Maastricht. https://doi.org/10.26481/dis.20200207gd

Document status and date:

Published: 01/01/2020

DOI:

10.26481/dis.20200207gd

Document Version:

Publisher's PDF, also known as Version of record

\section{Please check the document version of this publication:}

- A submitted manuscript is the version of the article upon submission and before peer-review. There can be important differences between the submitted version and the official published version of record.

People interested in the research are advised to contact the author for the final version of the publication, or visit the DOI to the publisher's website.

- The final author version and the galley proof are versions of the publication after peer review.

- The final published version features the final layout of the paper including the volume, issue and page numbers.

Link to publication

\footnotetext{
General rights rights.

- You may freely distribute the URL identifying the publication in the public portal. please follow below link for the End User Agreement:

www.umlib.nl/taverne-license

Take down policy

If you believe that this document breaches copyright please contact us at:

repository@maastrichtuniversity.nl

providing details and we will investigate your claim.
}

Copyright and moral rights for the publications made accessible in the public portal are retained by the authors and/or other copyright owners and it is a condition of accessing publications that users recognise and abide by the legal requirements associated with these

- Users may download and print one copy of any publication from the public portal for the purpose of private study or research.

- You may not further distribute the material or use it for any profit-making activity or commercial gain

If the publication is distributed under the terms of Article $25 \mathrm{fa}$ of the Dutch Copyright Act, indicated by the "Taverne" license above, 


\section{Myelin and Networks}

Magnetic Resonance Imaging in Epilepsy 
(C) 2020 Gerhard Sebastiaan Drenthen - All rights reserved

No part of this publication may be reproduced, stored in a retrieval system or transmitted in any form or by any means, electronic, mechanical, photocopying, recording or otherwise, without the prior permission of the author.

Printed by Gildeprint, the Netherlands

Layout by Gerhard Drenthen

Cover design by Gerhard Drenthen

Cover background image from https://pixabay.com/users/geralt-9301/

ISBN: 978-94-6380-691-6

Printing of this thesis was financially supported by Maastricht University 


\title{
Myelin and Networks
}

\section{Magnetic Resonance Imaging in Epilepsy}

\author{
PROEFSCHRIFT
}

ter verkrijging van de graad van doctor aan de Universiteit Maastricht, op gezag van de Rector Magnificus, Prof dr. Rianne M. Letschert volgens het besluit van het College van Decanen, in het openbaar te verdedigen

op vrijdag 7 februari 2020 om 14.00 uur

door

Gerhard Sebastiaan Drenthen

Geboren 27 januari 1989 te Almelo 


\section{Promotores}

Prof. dr. ir. W.H. Backes

Prof. dr. A.P. Aldenkamp

\section{Copromotor}

Dr. J.F.A. Jansen

\section{Beoordelingscommissie}

Prof. dr. F.M. Mottaghy (voorzitter)

Prof. dr. K.P.J. Braun (Universitair Medisch Centrum Utrecht)

Prof. dr. D.E.J. Linden

Prof. dr. ir. P.H.N. de With (Technische Universiteit Eindhoven) 


\section{Table of Contents}

Chapter 1: General introduction

Chapter 2: On the merits of non-invasive myelin imaging in epilepsy, a literature review.

Chapter 3: A new analysis approach for T2 relaxometry myelin-water quantification: orthogonal matching pursuit.

Chapter 4: Applicability and reproducibility of 2D multi-slice GRASE myelinwater fraction with varying acquisition acceleration.

Chapter 5: Lower myelin-water content of the frontal lobe in childhood absence epilepsy

Chapter 6: Constructing an axonal-specific myelin developmental atlas and its application to childhood absence epilepsy

Chapter 7: Functional brain integrity is related to epilepsy severity in childhood absence epilepsy.....

Chapter 8: Structural covariance networks relate to the severity of epilepsy with focal-onset seizures.

Chapter 9: General discussion

Summary 165

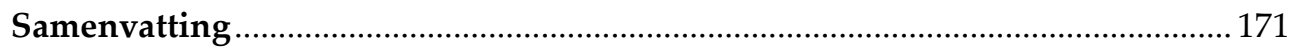

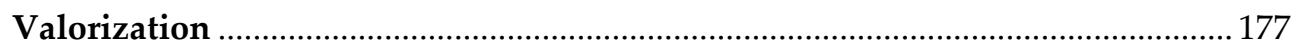

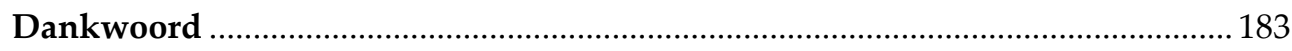

Curriculum Vitae ……………………………………………………………. 187

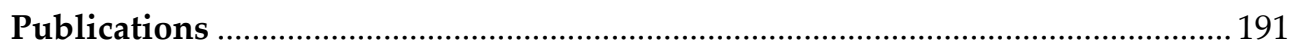





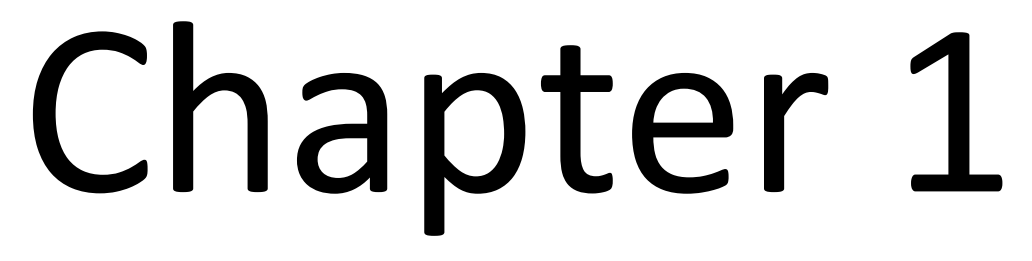

General Introduction 


\section{Epilepsy as a network disorder}

Epilepsy is one of the most common serious neurological disorders, with a world-wide prevalence of about 6 to 9 per 1000 persons [1]. The hallmark of epilepsy is recurrent epileptic seizures, which are defined as a temporary occurrence of signs and/or symptoms due to abnormal, excessive or synchronous neuronal activity in the brain. Epileptic seizures can be classified in three types, those with a focal onset, those with a (primarily) generalized onset, and those with an unknown onset. Besides the epileptic seizures, epilepsy is often accompanied by neurocognitive comorbidities [2].

Since the neuronal cell bodies that discharge synchronously during an epileptic seizure reside mainly in the gray matter (e.g. the cortex) of the brain, epilepsy was traditionally thought to be a disorder of the gray matter [3]. However, recent research has shown that the pathology of epilepsy is not limited to the gray matter, but rather affect the complex interactions of different brain regions connected through axon bundles in the white matter [4], [5]. This is one of the reasons that epilepsy is currently regarded as a disorder of the brain network as a whole.

The clinical diagnosis of epilepsy requires at least one unprovoked seizure and/or a risk $(>60 \%)$ of recurrent seizures or diagnosis of an epilepsy syndrome [6]. An epilepsy syndrome is characterized by a group of symptoms that share a common prognosis and response to treatment. Common examples of epilepsy syndromes are the West and Dravet syndromes and juvenile and childhood absence epilepsy. Childhood absence epilepsy (CAE) is characterized by frequent absence seizures resulting in brief losses of awareness in school-aged children. Absence seizures can occur multpile times a day, and show approximately $3 \mathrm{~Hz}$ generalized spike-and-wave discharges (GSWD) on an electroencephalogram (EEG). Neuroimaging of the brain is normally omitted from the standard clinical work-up as specific structural brain abnormalities are not regarded to be part of this generalized epilepsy syndrome. Nevertheless, cognitive [7] and neuroimaging [8]-[12] studies in children with absence epilepsy have raised concerns about this allegedly benign syndrome by investigating its true neurocognitive impact. A higher incidence of academic difficulties and neurocognitive deficits, mainly attentional deficits, have been previously observed in CAE [13]-[21].

\section{Myelin}

An important structural component for healthy neuronal maturation is the myelin sheath (Figure 1.1). The formation of myelin starts in the third gestational week and is a process that continues well into adulthood. The myelin sheath is wrapped around the axons by oligodendrocytes cells, which can myelinate dozens of axons simultaneously. The myelin comprises of $40 \%$ water, and the remaining $60 \%$ dry mass consists of $70-85 \%$ lipids and 15 $30 \%$ proteins [22]. The most important lipid is cholesterol, and the most important proteins 
are the myelin basic protein (MBP) and proteolipid protein (PLP). The myelin acts as an electrical insulator, allowing a more efficient and much faster conduction of electrical signals along the axons. There are gaps in the myelin sheath called nodes of Ranvier where an influx of sodium ions can occur to facilitate the propagation of electrical signals (i.e. action potential). The conduction velocity of the action potential moving along the unmyelinated gaps rapidly decreases due to the absence of the insulating myelin, therefore, the action potential appears to be jumping along the axon. This is referred to as saltatory conduction from the Latin saltare, which translates to 'to hop' or 'to leap'.

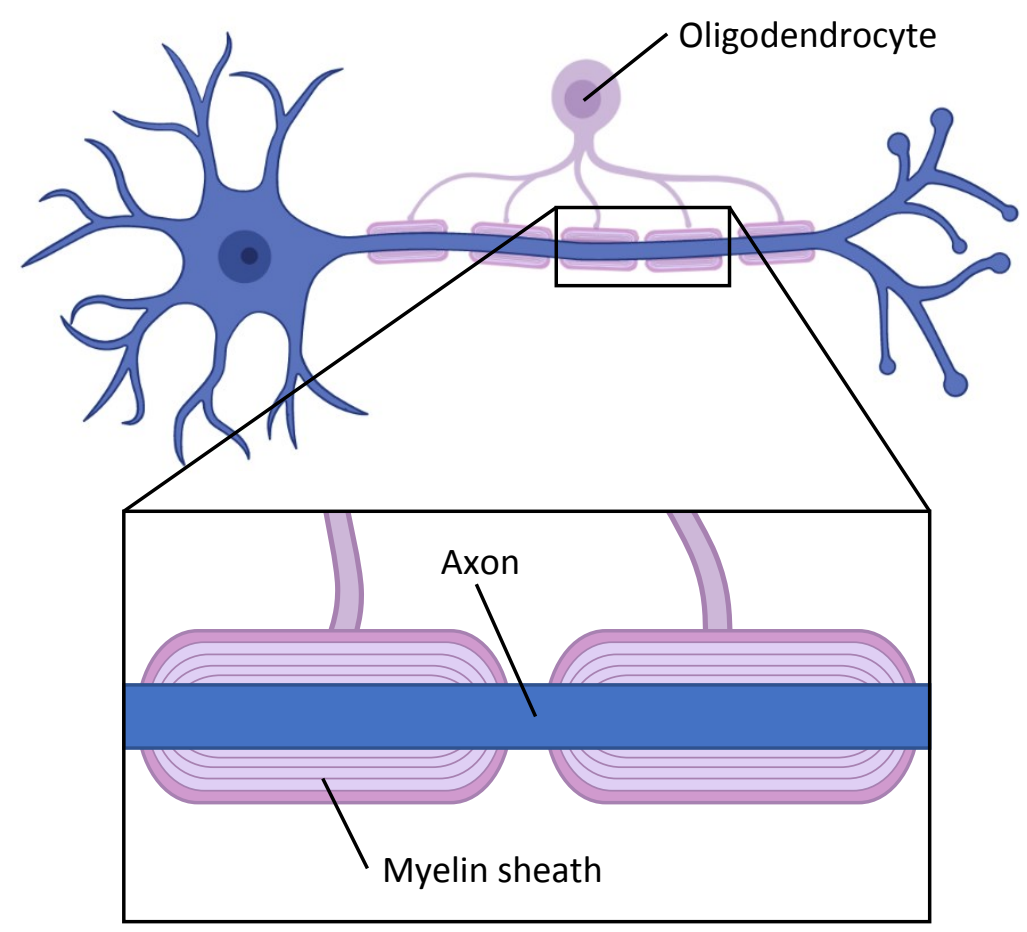

Figure 1.1: An illustrative view of a myelinated axon.

Myelin is a vital element of normal brain development. Abnormal myelination or demyelination is related to several neurological and neuropsychiatric disorders such as, Alzheimer's disease [23], multiple sclerosis (MS) [24], Parkinson's disease [25] and schizophrenia [26]. Moreover, abnormal myelin content has been recently reported for children with epilepsy [27], [28]. In vivo quantification of the myelin content using neuroimaging can provide useful insights into these disorders. Moreover, myelin imaging can be especially useful to study whether the epilepsy interferes with the normal development of myelin during the vulnerable years of childhood. 


\section{Intermezzo: Magnetic resonance imaging}

Magnetic resonance imaging (MRI) is a non-invasive imaging technique that can be used to create anatomical images of the body. When placing the human body in the strong magnetic field of an MRI scanner, the randomly oriented magnetic moments of the hydrogen protons in the body will spin out of phase, but with a specific frequency (i.e. Larmor frequency) and align either parallel or anti-parallel to the directions of the magnetic field. A slight surplus of protons will favour the parallel alignment, which is the lower energy state. By using specific radiofrequency pulses with the resonance frequency of hydrogen protons (i.e. Larmor frequency), the protons in the low energy state will be excited to a higher energy state, and furthermore, will start spinning in phase. After turning off the radiofrequency pulse, the excited protons will return to their equilibrium state through two separate relaxation processes, T1 and T2 relaxation. The T1 relaxation (i.e. longitudinal relaxation) relates to the time it takes for the excited protons to return to their equilibrium state in alignment with the external magnetic field. T2 relaxation (i.e. transverse relaxation) is a measure of time which relates to the loss of phase coherence. The relaxation times of specific hydrogen protons is related to their chemical environment, therefore, by applying the radiofrequency pulses in combination with local magnetic field gradients for spatial encoding, different types of tissue can be visualized.

\section{Myelin imaging}

Qualitative T2-weighted imaging provides excellent contrast to identify white matter abnormalities, however it does not provide quantitative measures of one of the most characteristic components of the white matter, the myelin content. Therefore, more specific techniques for myelin quantification have been proposed. For example, myelin-water imaging by means of T2-relaxometry is often used to provide a measure of the myelin content [29]. T2-relaxometry techniques are sensitive to differences in the mobility of water particles. More specific, the rotation of hydrogen protons causes small-scale local magnetic fields that interacts with other protons causing dephasing. For water particles with a more or less random mobility these effects tend to average out over space, resulting in a relatively long T2 relaxation time. However, when motion is restricted, the effect is averaged out less, resulting in shorter T2 relaxation times for bound water particles (e.g. water bound between the myelin sheath). Due to these differences in T2 relaxation time, MR imaging can provide surrogate markers of myelin content via the myelin-water, whereas the $\mathrm{T} 2$ relaxation profile of a single voxel is a superposition of several water components based on their mobility (e.g. myelin-water, intra-extra cellular water, and free water in the cerebrospinal fluid). The signal attributed to each of these components $(y)$ can then be determined using multi- 
exponential analysis by separating the measured signal into a spectrum of its constituting relaxation components $(s)$ using a predefined set of relaxation curves $(A)$ (Figure 1.2). From the resulting relaxation components, the fraction of myelin-water signal to the total water signal, the myelin-water fraction (MWF), can be determined.

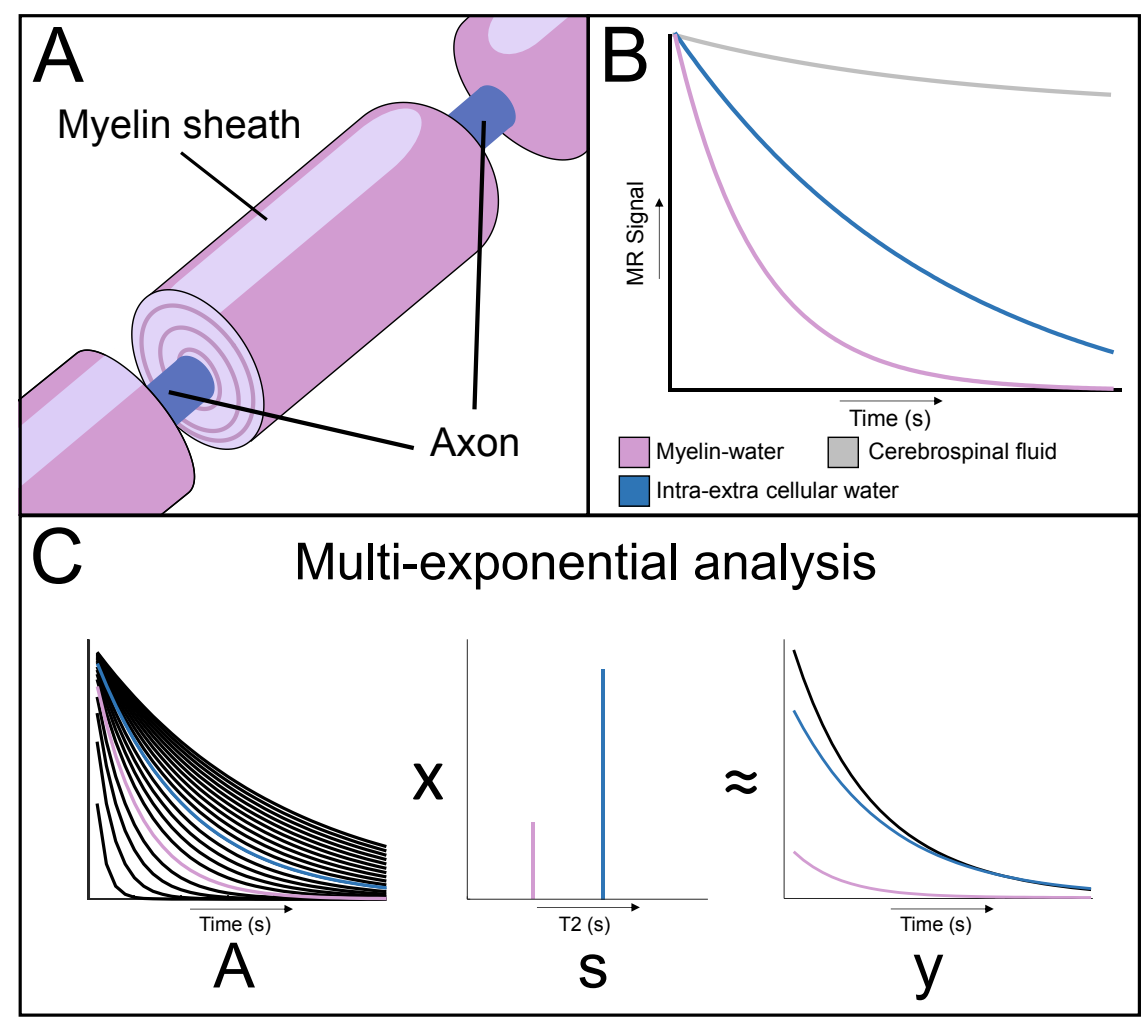

Figure 1.2: A) the myelin sheath spirals around the axon, and between the sheath layers water particles are trapped. B) Sample T2 relaxation profiles of the signal arising from the three water components are visualized. C) Graphical representation of the multi-exponential analysis. Using a predefined dictionary $\mathrm{A}$ the measured signal $\mathrm{y}$ is characterized by a distribution (s) of amplitudes. Blue represents the intra-extra cellular water compartment and purple the myelin-water compartment.

\section{Network imaging}

Besides quantifying local changes in the myelin content, the complex organisation of the white matter can also be assessed using MRI acquisitions based on the diffusion of water molecules. By estimating the directions of water diffusion in the white matter, bundles of white matter axons can be reconstructed by using tractography analysis. This enables the characterization of the structural brain network in terms of white matter tracts that 
interconnect distributed gray matter regions throughout the entire brain. While the diffusion of water molecules is related to the myelin content, it does not provide specific information on the myelin content and cannot discriminate between axonal injury, alterations in myelin content or topographic deviations at the microscopic scale [30]. Therefore, diffusion MRI is a complementary technique to myelin-water imaging.

Anatomical MRI images have been extensively applied to study cerebral abnormalities in patients with epilepsy, potentially aiding in diagnosis or eventual surgery preparation [31]. Furthermore, the epileptic brain often has other, more subtle abnormalities which are not visible at radiological inspection. To identify and understand these abnormalities, previous studies have focused on alterations in the thickness of the cortical gray matter of patients with epilepsy [32]-[34]. More recently, such morphological measurements at various brain regions have been shown to correlate between subjects. These correlations are thought to be part of an underlying anatomical network, the so-called structural covariance network ( $\mathrm{SCN})$. The SCN relies on the assumption that axonally connected regions have, to some extent, similar inter-subject variations and patterns of morphology [35].

Besides the macroscopic and microstructural components, brain areas are also known to show distinct temporal activity patterns in rest and during specific tasks using functional MRI (fMRI). When neuronal activity increases, oxygen is extracted from the haemoglobin proteins in the blood. This causes a local change in the ratio of oxygenated and deoxygenated haemoglobin. Oxygenated and deoxygenated haemoglobin have different magnetic properties, and a change in their ratio causes local field inhomogeneities which can be detected using magnetic susceptibility sensitive, i.e. T2*-weighted, imaging. Consequently, the $\mathrm{T}^{*}$-weighted signal is related to the blood responses to neuronal activity, also referred to as the blood-oxygen level dependent (BOLD) signal.

The topological organization of the complex structural and functional interactions throughout the distributed regions of the brain can be described as a network, where the brain regions represent nodes and their connections are the edges. Graph theoretical analysis can then be used to calculate certain network measures that characterize the brain network. Note that in case of fMRI, these connections do not describe the physical structural connections, but rather their temporal correlations (e.g. functional connectivity). Two of the most robust and widely applied global graph metrics are the characteristic path length and the clustering coefficient [36]. The path length gives insight into how well information can spread throughout the entire network (i.e. integration), while the clustering coefficient provides a measure of local information processing (i.e. segregation). 


\section{Aim and outline of this thesis}

The overall aim of this thesis is (i) to gain more insights into the underlying neurobiological substrate of epilepsy including its adverse cognitive side effect, with on the one hand an interest in the influence of myelin and on the other hand an interest in cerebral networks by (ii) using and advancing dedicated quantitative MR image techniques, focussed on image acquisition and analysis for the myelin content and cerebral networks.

To obtain a broad overview of the epileptic brain, advanced neuroimaging methods were employed, including various types of anatomical, microstructural, and functional imaging techniques and quantitative image analysis methods. More specifically, it was aimed to identify abnormalities in the brain that are related to epilepsy and its cognitive/behavioural comorbidities. To realize the tailoring and further development of novel MRI techniques to this disease, several cohorts of patients with epilepsy and healthy controls were used. New analysis and acquisition techniques were validated in healthy controls in chapters 3 and 4 . In chapters 5 through 7, children with CAE are included, while in chapter 8 adult patients with a focal seizure onset were studied.

The outline of the thesis is as follows; Chapter 2 provides an overview of the current evidence from literature on the involvement of myelin content in epilepsy. Evidence from preclinical and histological studies as well as neuroimaging studies are addressed.

Subsequently, in chapter 3, a new analysis method for myelin-water content estimation from the T2 relaxation decay curves is proposed: orthogonal matching pursuit (OMP). The OMP has a lower computational complexity compared to the commonly applied method non-negative least squares (NNLS), allowing for a denser set of relaxation functions for the multi-exponential analysis. The new analysis method is compared to the NNLS using simulations, phantom and in vivo data.

The reproducibility and scan time of an MRI technique have a direct implication on its clinical suitability. Therefore, in chapter 4, the applicability and reproducibility of an acquisition of myelin-water imaging data is presented. Moreover, the effect of a faster acquisition, through parallel imaging techniques, on the reproducibility is investigated.

In chapter 5 the myelin content in a sample of children with CAE and healthy age-sex matched controls is estimated using the new acquisition method. It is hypothesised that children with CAE have an abnormal myelin content, especially in the frontal areas.

In chapter 6 the development of axonal-specific myelin content is assessed in healthy controls by means of diffusion MRI and myelin-water imaging. Subsequently, the applicability of the resulting myelin developmental atlas is established in children with CAE.

Besides these structural metrics, in chapter 7 the functional network of children with CAE is determined and characterized. The network is quantified using graph theoretical analysis and subsequently related to clinical characteristics of children with CAE. 
In chapter 8 individual SCNs of adult patients with focal seizure onset are calculated. This chapter mainly focusses on the relation of individual SCNs and epilepsy severity and its cognitive comorbidities in adulthood.

Last, in chapter 9 the findings of this thesis are discussed and integrated in a broader context and provide directions for future research. 


\section{References}

[1] K. M. Fiest et al., "Prevalence and incidence of epilepsy A systematic review and metaanalysis of international studies," Neurology, vol. 88, pp. 296-303, 2017.

[2] J. J. Lin, M. Mula, and B. P. Hermann, "Uncovering the Lifespan Neurobehavioral Comorbidities of Epilepsy," Lancet, vol. 380, no. 9848, pp. 1180-1192, 2013.

[3] E. H. Reynolds, "Jackson, Todd, and the Concept of 'Discharge ' in Epilepsy," vol. 48, no. 11, pp. 2016-2022, 2016.

[4] F. Deleo, M. Thom, L. Concha, A. Bernasconi, B. C. Bernhardt, and N. Bernasconi, "Histological and MRI markers of white matter damage in focal epilepsy," Epilepsy Res., vol. 140, pp. 29-38, 2018.

[5] W. M. Otte, P. Van Eijsden, J. W. Sander, J. S. Duncan, R. M. Dijkhuizen, and K. P. J. Braun, "A meta-analysis of white matter changes in temporal lobe epilepsy as studied with diffusion tensor imaging," Epilepsia, vol. 53, no. 4, pp. 659-667, 2012.

[6] R. S. Fisher et al., "A practical clinical definition of epilepsy," Epilepsia, vol. 55, no. 4, pp. 475482, 2014.

[7] A. Verrotti, S. Matricardi, V. E. Rinaldi, G. Prezioso, and G. Coppola, "Neuropsychological impairment in childhood absence epilepsy: Review of the literature," J. Neurol. Sci., vol. 359, no. 1-2, pp. 59-66, 2015.

[8] R. Caplan et al., "Frontal and temporal volumes in Childhood Absence Epilepsy," Epilepsia, vol. 50, no. 11, pp. 2466-2472, 2009.

[9] G. Di Wang et al., "Grey matter anomalies in drug-naïve childhood absence epilepsy: A voxelbased morphometry study with MRI at 3.0 T," Epilepsy Res., vol. 124, pp. 63-66, 2016.

[10] J. S. Liang et al., "Microstructural Changes in Absence Seizure Children: A Diffusion Tensor Magnetic Resonance Imaging Study," Pediatr. Neonatol., vol. 57, no. 4, pp. 318-325, 2016.

[11] K. Xue et al., "Diffusion tensor tractography reveals disrupted structural connectivity in childhood absence epilepsy," Epilepsy Res., vol. 108, no. 1, pp. 125-138, Jan. 2014.

[12] W. Qiu et al., "Structural Abnormalities in Childhood Absence Epilepsy: Voxel-Based Analysis Using Diffusion Tensor Imaging," Front. Hum. Neurosci., vol. 10, p. 483, 2016.

[13] D. Cheng, X. Yan, Z. Gao, K. Xu, X. Zhou, and Q. Chen, "Common and Distinctive Patterns of Cognitive Dysfunction in Children With Benign Epilepsy Syndromes," Pediatr. Neurol., vol. 72, pp. 36-41, Jul. 2017.

[14] D. Masur et al., "Pretreatment cognitive deficits and treatment effects on attention in childhood absence epilepsy," Neurology, vol. 81, no. 18, pp. 1572-1580, 2013.

[15] C. L. Kernan et al., "Neurocognitive profiles in children with epilepsy," Epilepsia, vol. 53, no. 12, pp. 2156-2163, 2012.

[16] C. Cerminara et al., "Attention impairment in childhood absence epilepsy: An impulsivity problem?," Epilepsy Behav., vol. 27, no. 2, pp. 337-341, 2013.

[17] E. D'Agati, C. Cerminara, L. Casarelli, M. Pitzianti, and P. Curatolo, "Attention and executive functions profile in childhood absence epilepsy," Brain Dev., vol. 34, no. 10, pp. 812-817, 2012.

[18] M. Levav, A. F. Mirsky, J. Herault, L. Xiong, N. Amir, and E. Andermann, "Familial Association of Neuropsychological Traits in Patients With Generalized and Partial Seizure Disorders," J. Clin. Exp. Neuropsychol., vol. 24, no. 3, pp. 311-326, May 2002.

[19] A. T. Berg, S. R. Levy, F. M. Testa, and H. Blumenfeld, "Long-term seizure remission in childhood absence epilepsy: Might initial treatment matter?," Epilepsia, vol. 55, no. 4, pp. 551557, 2014.

[20] K. J. Oostrom, A. Smeets-Schouten, C. L. J. J. Kruitwagen, A. C. B. Peters, and A. JennekensSchinkel, "Not Only a Matter of Epilepsy: Early Problems of Cognition and Behavior in Children With 'Epilepsy Only'--A Prospective, Longitudinal, Controlled Study Starting at Diagnosis," Pediatrics, vol. 112, no. 6, pp. 1338-1344, 2003. 
[21] A. Covanis, K. Skiadas, N. Loli, C. Lada, and V. Theodorou, "Absence epilepsy: early prognostic signs," Seizure Eur. J. Epilepsy, vol. 1, no. 4, pp. 281-289, 1992.

[22] P. Morell and R. Quarles, "Characteristic Composition of Myelin," in Basic Neurochemistry: Molecular, Cellular and Medical Aspects, 6th ed., G. Siegel, B. Agranoff, R. Albers, and P. Molinoff, Eds. Philadelphia, 1999.

[23] E. Kavroulakis et al., "Myelin content changes in probable Alzheimer's disease and mild cognitive impairment: Associations with age and severity of neuropsychiatric impairment," J. Magn. Reson. Imaging, vol. 47, no. 5, pp. 1359-1372, 2018.

[24] S. Kolind et al., "Clinical Brain and cord myelin water imaging: a progressive multiple sclerosis biomarker," NeuroImage Clin., vol. 9, pp. 574-580, 2015.

[25] D. C. Dean III et al., "Alterations of Myelin Content in Parkinson's Disease : A Cross-Sectional Neuroimaging Study," PLoS One, vol. 11, no. 10, p. e0163774, 2016.

[26] D. J. M. Lang et al., "Clinical 48 echo T2 myelin imaging of white matter in first-episode schizophrenia: Evidence for aberrant myelination," NeuroImage Clin., vol. 6, pp. 408-414, 2014.

[27] H. S. Spader et al., "Advances in myelin imaging with potential clinical application to pediatric imaging," Neurosurg. Focus, vol. 34, no. 4, p. E9, Apr. 2013.

[28] K. Moldovan et al., "Myelin water fraction changes in febrile seizures," Clin. Neurol. Neurosurg., vol. 175, pp. 61-67, 2018.

[29] E. Alonso-Ortiz, I. R. Levesque, and G. B. Pike, "MRI-based myelin water imaging: A technical review," Magn. Reson. Med., vol. 73, no. 1, pp. 70-81, Jan. 2015.

[30] H. Chahboune, A. M. Mishra, M. N. Desalvo, L. H. Staib, M. Purcaro, and D. Scheinost, "DTI abnormalities in anterior corpus callosum of rats with spike - wave epilepsy," Neuroimage, vol. 47, pp. 459-466, 2009.

[31] F. Cendes, W. H. Theodore, B. H. Brinkmann, V. Sulc, and G. D. Cascino, "Neuroimaging of epilepsy," Handb Clin Neurol., vol. 136, pp. 985-1014, 2016.

[32] E. Widjaja, S. Z. Mahmoodabadi, O. C. Snead, A. Almehdar, and M. Lou Smith, “Widespread cortical thinning in children with frontal lobe epilepsy," Epilepsia, vol. 52, no. 9, pp. 16851691, 2011.

[33] G. M. Overvliet et al., "Early onset of cortical thinning in children with rolandic epilepsy," NeuroImage Clin., vol. 2, pp. 434-439, 2013.

[34] R. M. H. Besseling et al., "Delayed convergence between brain network structure and function in rolandic epilepsy," Front. Hum. Neurosci., vol. 8, p. 704, 2014.

[35] B. C. Bernhardt, Z. Chen, Y. He, A. C. Evans, and N. Bernasconi, "Graph-Theoretical Analysis Reveals Disrupted Small-World Organization of Cortical Thickness Correlation Networks in Temporal Lobe Epilepsy," Cereb. Cortex, vol. 21, no. 9, pp. 2147-2157, Sep. 2011.

[36] D. J. Watts and S. H. Strogatz, "Collective dynamics of 'small-world' networks," Nature, vol. 393, no. 6684, pp. 440-442, Jun. 1998. 



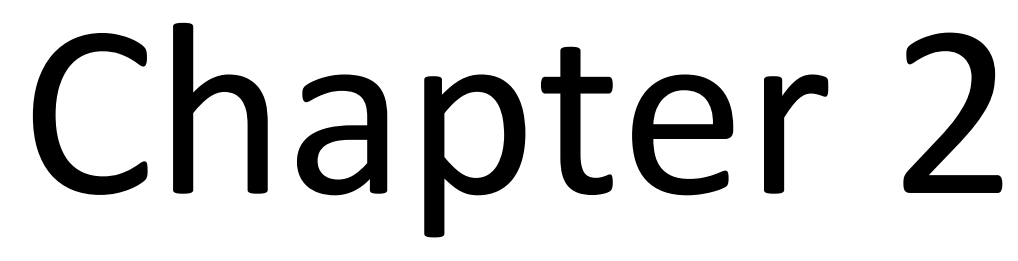

\section{On the merits of non-invasive myelin imaging in epilepsy, a literature review}

Gerhard S. Drenthen, Walter H. Backes, Albert P. Aldenkamp, R. Jeroen Vermeulen, Sylvia Klinkenberg, Jacobus F.A. Jansen 


\section{Abstract}

Myelin is a vital element of normal brain development and structure. Myelination is most prominent during first two years of life and proceeds until the age of 30. Abnormal myelination is related to several neurological and neuropsychiatric disorders such as Alzheimer's disease and multiple sclerosis. Recently, abnormal myelin content has been reported in children with epilepsy. Furthermore, more and more literature hints at a link between abnormal myelination and epilepsy, hence it is worthwhile to evaluate the benefits of non-invasive myelin imaging.

In this literature review, we provide an overview of the current evidence of myelin abnormalities in epilepsy from imaging and histological studies. After preselection, 21 histological and 21 in vivo imaging studies were identified. Primarily, epilepsy is found to be associated with a reduced myelin content. However, more studies that apply myelin-specific imaging techniques are needed to determine whether the myelin abnormalities are an underlying cause of epilepsy, or a consequence of the excessive activity in epilepsy. 


\section{Introduction}

A structural component of brain tissue that is of crucial importance for healthy neuronal maturation is the myelin sheath. The formation of myelin starts in the third gestational week and is a process that continues well into adulthood [1]. The myelin sheath is wrapped around the axons by oligodendrocytes cells, which can myelinate dozens of axons simultaneously. In Figure 2.1 an electron microscopy image (3000x magnified) of the myelin wrapped around the axons is shown. The myelin comprises of water for $40 \%$, and the remaining $60 \%$ dry mass consists for $70-85 \%$ of lipids and $15-30 \%$ of proteins [2]. The most important lipid is cholesterol and the most important proteins are myelin basic protein (MBP) and proteolipid protein (PLP).

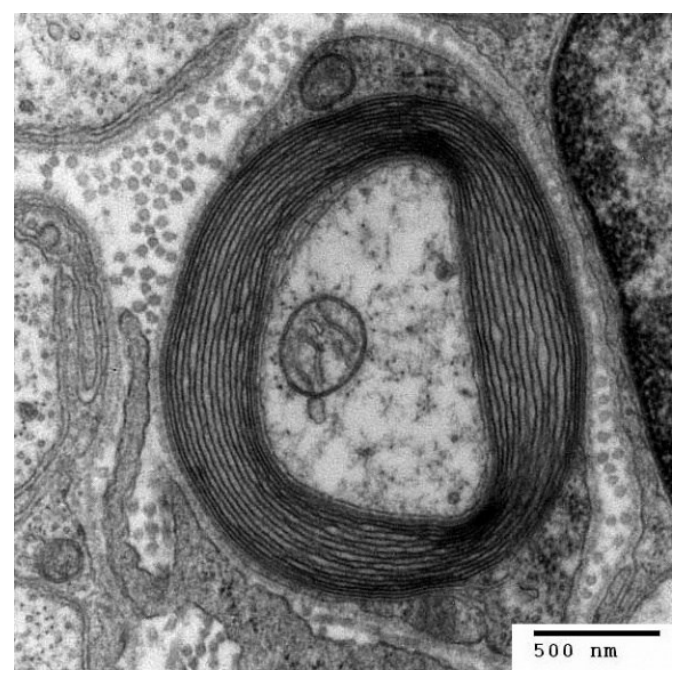

Figure 2.1: Transmission electron micrograph of a myelinated axon. The myelin layer (concentric) surrounds the axon of a neuron, showing cytoplasmatic organs inside. Generated and deposited into the public domain by the Electron Microscopy Facility at Trinity College.

Myelin acts as an electrical insulator, allowing a more efficient and much faster conduction of electrical signals along the axons. There are gaps in the myelin sheath called nodes of Ranvier where the influx of sodium ions occurs to facilitate the propagation of electrical signals (i.e. action potential). The conduction velocity of the action potential moving along the gaps rapidly decreases due to the absence of the insulating myelin. Therefore, the action potential appears to be jumping along the axon, which is referred to as saltatory conduction from the Latin saltare and translates to 'to hop' or 'to leap'. Myelin is a vital element of normal brain development. Abnormal myelination is related to several neurological and neuropsychiatric disorders such as, Alzheimer's disease [3], multiple sclerosis (MS) [4], Parkinson's disease [5] 
and schizophrenia [6]. Moreover, abnormal myelin content has been recently reported for children with epilepsy [7], [8].

Epilepsy is one of the most common neurological disorders, with a prevalence of about 6 to 9 per 1000 persons [9]. The hallmark of epilepsy is recurrent epileptic seizures, which are defined as a temporary occurrence of signs and/or symptoms due to abnormal, excessive or synchronous neuronal activity in the brain. Epilepsy is clinically diagnosed after at least one unprovoked seizure and a risk (> 60\%) of recurrent seizures or after diagnosis of an epilepsy syndrome [10]. Well-known examples of epilepsy syndromes are the West syndrome (i.e. infantile spasms), childhood absence epilepsy (CAE) and Dravet syndrome. Epileptic seizures are classified into three types, those with a focal onset, those with a (primarily) generalized onset and those with an unknown onset.

Since the neuronal cell bodies that discharge synchronously during an epileptic seizure reside mainly in the gray matter of the brain, epilepsy was traditionally thought to be a disorder of the gray matter [11]. However, recent research has shown that the disruptions of brain tissue in epilepsy are not limited to the gray matter, but rather affect the complex interactions of different brain regions connected by via the fiber bundles of the white matter. Therefore, epilepsy is currently regarded as a brain network disorder, for which the white matter also plays an important role [12].

In this literature review, we first provide an overview of the current evidence of myelin abnormalities in epilepsy from preclinical investigations and histological studies on human tissue samples. Second, we outline the studies that have investigated myelin content in patients with epilepsy using non-invasive imaging methods. Third and last, we discuss future promising methods and research opportunities in the context of epilepsy and myelin.

\section{Myelin in epilepsy}

The underlying mechanisms of epilepsy and its characteristic seizures are not yet completely understood. While the etiology can be identified in quite a number of patients with epilepsy, with for instance traumatic brain injury-related developmental anomalies of the brain and genetic causes such as mutations in sodium channels, there remain many patients for whom the cause of the epilepsy remains unknown [13]. It is assumed that during the development of epilepsy, e.g. epileptogenesis, the normal brain transitions into one that can suffer from hyperexcitability leading to an epileptic seizure. Possible underlying factors of a hyperexcitable state of the brain are numerous, including increases in excitatory or decreases in inhibitory synaptic neurotransmission, alterations in voltage gated channels or intra- extra cellular ion concentrations, or as a result of several synchronous subthreshold excitatory stimuli allowing their temporal summation in the post synaptic neurons [14]. This summation could be a direct consequence of axons with poorly distributed conduction velocities that result in the synchronous firing of action potentials. The conduction velocity of an axon is mainly related to its diameter and the myelin sheath. Therefore, a direct relation might exist 
between epileptic seizure susceptibility and an abnormal myelin content. Conversely, previous studies indicated that neurological disorders associated with an abnormal myelin content are accompanied with a higher susceptibility to epileptic seizures [15]-[18]. For example, MS patients are three to six times more likely to develop epileptic seizures [19]. This further indicates that myelin might be related to the epileptogenesis and epileptic seizures. Therefore, advanced non-invasive neuroimaging methods specifically targeted to visualize the myelin sheath may provide new insights into the underlying mechanisms of epileptic seizures. While the white matter microstructure in patients with epilepsy has already been studied to some extent in terms of mobility and anisotropy of diffusing water molecules [12], [20], only few studies have focussed specifically on the myelin content [7], [8].

\section{Search criteria}

In this study, we investigated the presence of myelin dysfunction in epilepsy through a literature review, by focusing on the subject of myelin integrity measurements in epilepsy. We searched PubMed for articles which had the following terms in either the title, abstract or as a keyword: "epilepsy" or "seizure", and "myelin" or "myelination" or "demyelination", excluding non-English articles and reviews. We cross-referenced the selected articles and other reviews to include as many relevant studies as possible. The PubMed search resulted in 536 hits. After reading the title and abstract of each article, 21 histological and 21 imaging studies remained, of which 1 study overlapped between histological and imaging.

\section{Search results}

The selected histological studies are shown in Table 2.1, and the selected imaging studies are shown in Table 2.2. 


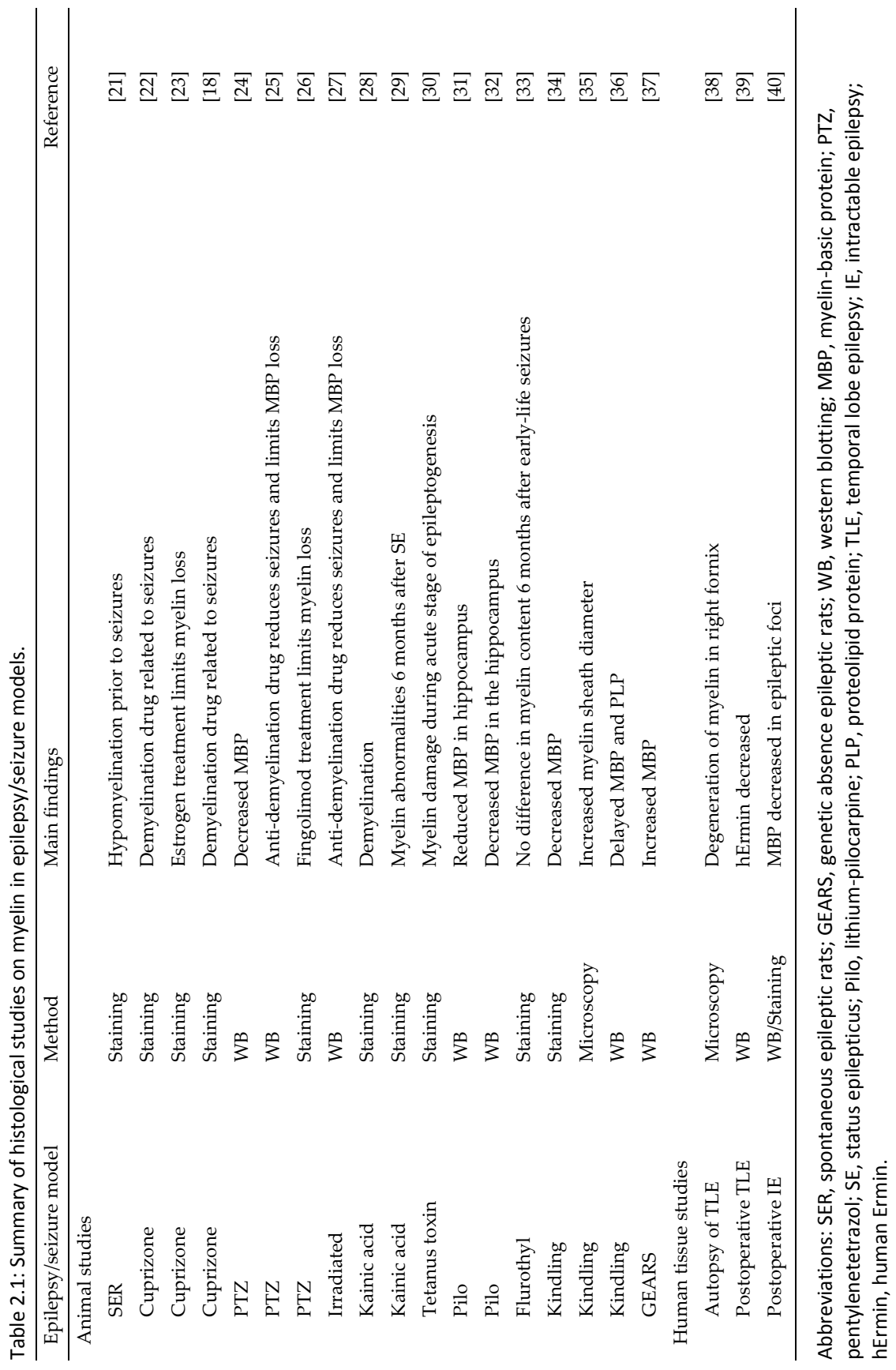




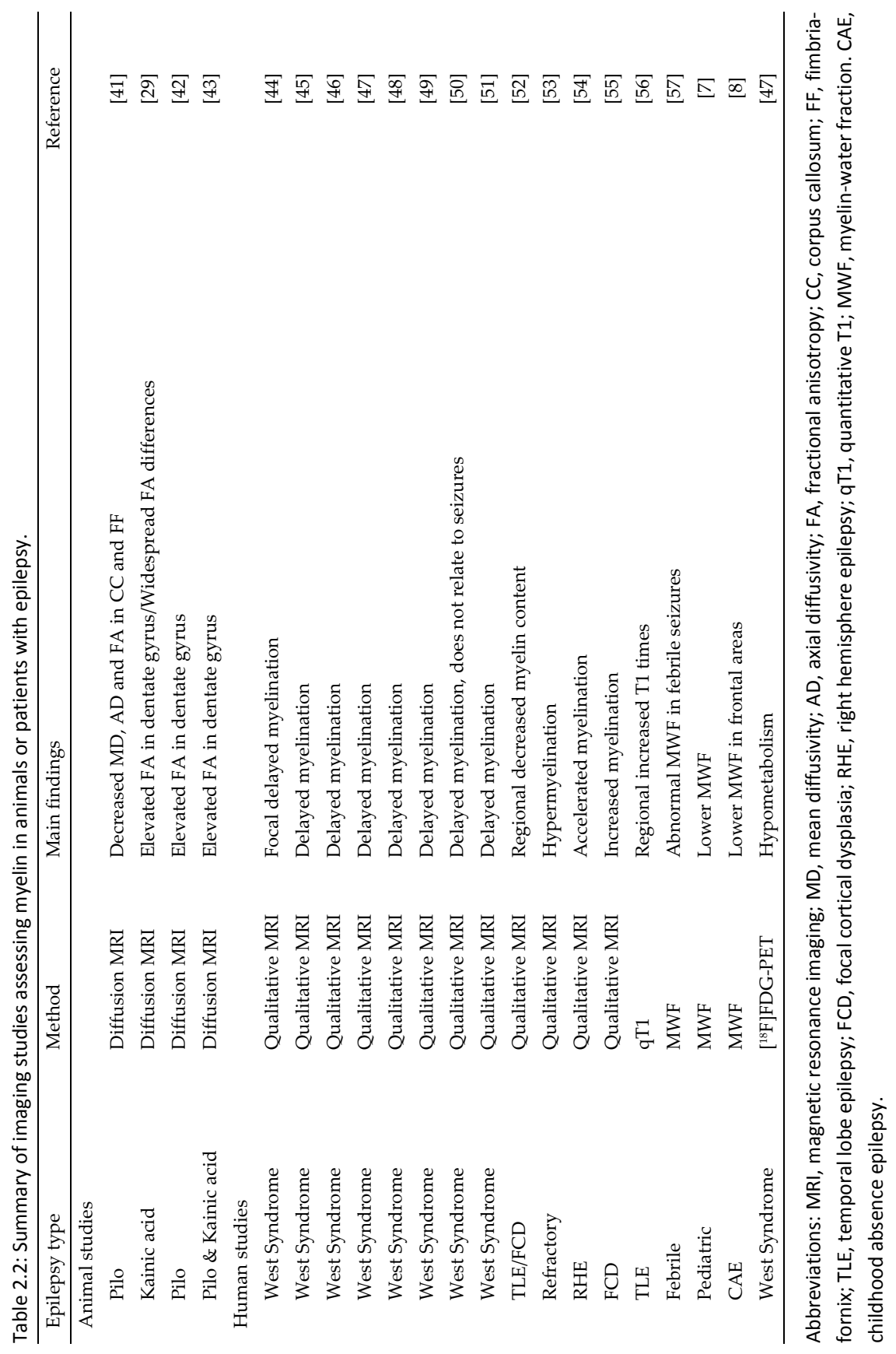




\section{Evidence from histological studies}

The myelin content has previously been assessed in preclinical and post-mortem studies using histological assessment. There are several methods to study myelin. For example, specific myelin-related proteins can be detected in tissue using Western Blotting and immunohistochemistry, while the proteins and lipids of the myelin sheath can also be stained, or the sheath can be visually inspected directly using electron microscopy.

\section{Animal studies}

Already in 1990, Inui et al. demonstrated hypomyelination in a mutant of the "zitter" and "tremor" rats using Luxol fast blue and Sudan black B staining [21]. This mutant was dubbed the spontaneous epileptic rat (SER), since these rats exhibit frequent and spontaneous absence-like and tonic convulsive seizures. The SER was also considered a useful model to study epilepsy since administration of antiepileptic drugs (AED) could inhibit its seizures [58], [59]. Furthermore, using electron microscopy, vacuoles were found in the tracts. These vacuoles were closely related to the hypomyelination and the reduction of myelin occurred prior to the onset of seizures. This suggest that vacuolation and/or hypomyelination are prerequisites for the seizure-inducing pathological condition.

Another method to study the effects of reduced myelin content and seizures is the administration of demyelinating toxins. For instance, previously, studies have investigated the effect of administrating cuprizone in rats. The cuprizone model is a model of toxic demyelination where copper chelator cuprizone (bis-cyclo-hexanone-oxaldihydrazone) is fed to the animals which subsequently leads to consistent demyelination. This model is especially useful for studying factors, such as drugs that could prevent the demyelination [60]. Using immunohistochemistry, it was shown that long-term administration of cuprizone does indeed reduces the myelin content (PLP and MBP) and relates to the occurrence of spontaneous seizures [18], [22]. Taylor et al. have shown that the cuprizone-induced demyelination can partially be prevented by the administration of estrogen (17 $\beta$-estradiol) [23]. Although the cuprizone rat model is better suited as comparison with MS rather than epilepsy in humans, these studies do provide insights in the relation between demyelination and susceptibility to seizures.

In a similar way, the effect of anti-demyelination drugs was previously studied in rats with pentylenetetrazol (PTZ)-induced seizures [25], [26] and in utero irradiated rats with malformations of cortical development [27]. In these studies, the administration of either fingolimod, quetiapine or glatiramer acetate related to myelin sheath protection and fewer seizures, which further establishes a foundation for either demyelination-induced seizures or a seizure-induced demyelination. PTZ-induced epilepsy in rats is a representative model for human epilepsy, and has previously been associated with damage to the myelin sheath in an early stage after administration [24]. 
Furthermore, systematic administration of kainic acid, a naturally occurring acid in seaweed and an analogue of excitotoxic glutamate, is widely applied as a model of epileptic seizures and neuronal degeneration [61]. Hopkins et al. studied the temporal progression $(2,4,8,24$, $48 \mathrm{~h}, 2$ weeks and 2 months) of myelin changes after a single injection of kainic acid in adult rats [28]. Within an hour of injection, the rats exhibited seizures lasting for up to 3 hours, after which the seizures did not reoccur. After 2 days, the myelin content changed to a more fragmented appearance. By 2 months, some remyelination occurred. Furthermore, Sierra et al. showed that 6 months after kainic-induced status epilepticus, myelin abnormalities could still be observed, showing less dense myelinated fibre packing in the entorhinal cortex while the fibres in the thalamus were more densely packed [29]. This indicated that after the seizure induction the damage to the myelin sheath did not worsen over time, while the myelin damage could not be completely restored.

Using a local administration of tetanus toxin directly on the cortex through an incision on the skull, an accurate model of chronic focal neocortical epilepsy can be induced in rats [62]. Using immunohistochemistry the PLP was shown to be decreased in the hippocampus after 10 weeks of seizure induction [30]. Besides the myelin loss in the hippocampus, this brain structure also showed structural alterations that resembled hippocampal sclerosis, which is a distinctive feature of temporal lobe epilepsy. The lithium-pilocarpine (pilo) model is often used as a model for temporal lobe epilepsy. Previously, reductions in MBP, myelin volume and thickness in the hippocampus were associated with the pilo model [31], [32]. The corresponding damage to the myelin sheath was shown to occur during the acute phase of the epileptogenesis, and the damage increased throughout the trajectory of epilepsy [32]. Furthermore, accompanying the myelin damage, the number of oligodendrocyte cells was found to be decreased, suggesting that the myelin decrease partly happens due to oligodendrocyte cell death. This indicated that the reduced myelin content or oligodendrocyte cell loss in the hippocampus is possibly related to the duration of epilepsy.

Barry et al. investigated the effect of early-life seizures on cognitive performance and myelination. The early-life seizures were induced in rat pups by letting them inhale flurothyl [33]. Six months after the induced early-life seizures the flurothyl treated rats showed cognitive deficits compared to control rats. However, no myelin abnormalities were found as assessed by staining. This indicates that the apparent cognitive deficits are most likely not related to the myelin content, and that there is no long-term effect of early-life seizures on myelination.

Another model for the development of seizures and epilepsy is kindling. Seizures are induced, often via direct electrical activity using electrodes implanted in the brain (e.g. amygdala), which increases the likelihood (i.e. lowers the threshold) for more subsequent seizures. Previously, in an electrical kindling rat model, a decreased MBP was reported using immunohistochemistry [34]. Moreover, the epileptic rats presented with learning and memory problems, which were likely associated to the demyelination. Furthermore, in a genetic seizure prone (e.g. fast kindling, dubbed FAST strain) rat model, reduced MBP and 
PLP were reported compared to seizure resistant rats (e.g. slow kindling, dubbed SLOW strain) [36]. Interestingly, the reductions of MBP and PLP were observed in the FAST strain even without inducing seizures by electrical kindling, indicating that seizure activity did not cause the reduction in myelin content. In a similar model (FAST and SLOW), an increased myelin sheath thickness was observed in the seizure prone (FAST) rats [35]. This increase was suggested to cause increased strain on the nodes of Ranvier, affecting the overall strength of the axonal integrity [35]. Previously, in genetic absence epileptic rats (GEARS), a similar finding of myelin sheath swelling and MBP upregulation was reported in the thalamus [37]. Swelling of the myelin sheath was suggested to affect the overall efficiency of action potential propagation, while the upregulation of MBP was suggested to lead to hyperpolarization in the thalamus and the generation and maintenance of spike-and-wave activity [63].

\section{Post-mortem evidence}

Extending upon the preclinical histology studies, the myelin content has also been studied in post-operative brain tissue from patients with intractable epilepsy. Hu et al. reported that the MBP was reduced in epileptic tissue compared to normal brain tissue [40]. Furthermore, visual inspection of the myelin fibers also revealed differences, being more loose and surrounded by vacuoles compared to the normal tissue [40]. In contrast, post-operative brain tissue from patients with intractable temporal lobe epilepsy did not show a reduction of PLP [39]. However, a specific oligodendrocyte protein, hErmin, did show a significant reduction in the epileptic temporal lobe compared to normal brain tissue. This finding suggests that myelin and/or oligodendrocytes cells are involved in the epileptogenic process.

Focal cortical dysplasia (FCD) presents as a malformation of cortical development, which is a common cause of intractable epilepsy. Post-operative brain tissue from FCD patients was previously shown to exhibit reductions of oligodendroglia cells [64], myelin content [65], as well as a relation between the myelin reduction and the duration of epilepsy [66]. Previously, Deleo et al. provided a review of the literature on the involvement of the myelin content in FCD [67].

Furthermore, autopsies in patients with right temporal lobe epilepsy showed degeneration of myelin in the right fornix compared to non-epileptic autopsies [38]. The number of myelinated fibres did not differ between the groups, whereas the number of nonmyelinated fibres was significantly reduced in fornix of the autopsies in patients with temporal lobe epilepsy. 


\section{Evidence from imaging studies}

\section{Qualitative MRI}

While histological and animal studies are invaluable to the ongoing research of myelin abnormalities and epilepsy, it remains a challenge to relate the findings directly to the in vivo tissue of humans. To this end, non-invasive methods to measure the myelin content in vivo are relevant to further elucidate the relation between myelin and epilepsy. For instance, the white matter is relatively bright on T1-weighted images which is related to myelin-bound cholesterol, while the T2-weighted contrast of white matter is low due to motion-restricted protons in the myelin-water. Structural T1- and T2-weighted magnetic resonance imaging (MRI) has already been extensively applied to study myelination changes in West syndrome. West syndrome is a relatively rare type of epilepsy, with a broad etiology ranging from genetic causes to severe neonatal brain injury with an incidence of $2-5$ per 10.000 , and it is characterized by epileptic spasms in infants [68]. It is reported that these infants exhibit delayed myelination patterns [44]-[46], [48]-[51], [69], [70], although, the delayed myelination did not relate to the seizure outcome [50]. More recently is was observed that blurring of the gray-white matter boundaries and regional decreases in myelination occurred in temporal lobe epilepsy [52].

While most reports on myelin and epilepsy or seizures concern a decreased myelin content, an elevated myelin content has also been reported in some extreme cases of epilepsy. For example, hypermyelination was observed in a case report of a woman with mental retardation and severe refractory epilepsy [53]. Additionally, a case report described an infant with hemimegalencephaly and severe right-sided epilepsy, accompanied with accelerated myelination in the same hemisphere [54]. This suggests that abnormal myelination might be related to the epileptic activity. Furthermore, in four infant cases of focal cortical dysplasia, the T1- and T2-weighted MRI signals were consistent with increased myelination [55].

\section{Quantitative MRI}

In contrast to standard structural MRI, diffusion weighted imaging (DWI) can provide information on the microstructure of the white matter tracts [71], which may be indirectly related to abnormalities in the myelin content. There has been extensive research on white matter abnormalities in epilepsy assessed with DWI, for example, from animal studies. Six months after inducing a status epilepticus in rats using pilocarpine [42] or kainic acid [29], ex vivo diffusion tensor imaging (DTI) of the hippocampus showed distinct structural changes. For example, the dentate gyrus showed an elevated fractional anisotropy due to an increase of mossy fibres connecting the dentate gyrus to the hippocampal subfield CA3. This sprouting of mossy fibres was previously linked to epileptogenesis [72]. Moreover, a similar finding of elevated fractional anisotropy was reported using in vivo DTI in a combination of kainic and 
pilo induced status epilepticus in rats [43]. These results were substantiated by a myelin increase observed through staining in the dentate gyrus. Furthermore, decreased in fractional anisotropy, mean and axial diffusivity were reported in the fimbriae fornix and corpus callosum of rats with pilo induced status epilepticus [41]. Similarly, to the previous studies, these results were also in line with a reduction of myelin observed with staining. Besides these animal studies, there is extensive literature available that shows a disrupted structural microstructure in patients with focal and generalized epilepsies, for reviews see Otte et al. [12] and Slinger et al. [20]. While the existing literature emphasizes the relevance of investigating the white matter microstructure in epilepsy, it fails to provide specific markers for the myelin content [73]. Correlations between diffusion MRI and histopathology (staining) did not find a consistent relation of myelin-content and MRI measures. In addition, resected brain tissue from intractable temporal lobe epilepsy patients showed an inverse relation between the thickness of the myelin sheath and fractional anisotropy (FA) [74]. Contrarily, it was shown that the MBP did neither relate to diffusion MRI measures nor to the signal intensity of FLAIR images [75]. This indicates that while the myelin content does influence the FA, the FA is not highly specific as a marker for myelin content, but relates also to the microscopic (geometric) water diffusion boundaries.

Reeves et al. showed that the MBP, estimated using immunohistochemistry, of ex vivo post-operative brain tissue related to the $\mathrm{T} 1$ and $\mathrm{T} 2$ relaxation times estimated using high field MRI (9.4T) [76]. They suggested that the $\mathrm{T} 1$ and $\mathrm{T} 2$ relaxation times might provide a more direct measure of the myelin sheath compared to diffusion MRI measures of water molecules. For example, the T1 relaxation time is known to be inversely related to the relatively high concentration of cholesterol in myelin [77]. A study using quantitative T1 relaxation time mapping in temporal lobe epilepsy revealed increased T1 relaxation times in temporopolar, parahippocampal and orbitofrontal cortices [56], possibly reflecting a decreased myelin content. Furthermore, the T2 relaxation time of white matter tissue is closely related to motion-restricted water protons in the myelin(-water). The water trapped between the layers of the myelin sheath has a different T2 decay compared to the more freely moving cellular water. Using complex computational multi-exponential analysis, the myelin-water can be distinguished from the more freely moving cellular water to obtain the so-called myelin-water fraction (MWF). As such, MR imaging can provide a surrogate marker of the myelin content via the myelin-water [78]. For a comprehensive technical review on MRI-based myelin-water imaging (MWI) see Alonso-Ortiz et al. [78]. In Figure 2.2 an axial slice of a T1-weighted image and MWF map is shown for an adult male subject.

Selected pediatric studies have already investigated the myelin-content using MWI in relation to epilepsy and seizures. Moldovan et al. examined the MWF in children with febrile seizures and found distinct changes in myelin-water content [57]. Children with simple febrile seizures showed an increased MWF compared to controls but did not develop epilepsy afterwards. However, the children with complex febrile seizures showed a decreased MWF and later developed epilepsy. This indicates that abnormal (either elevated or decreased) 
myelin-content can contribute to the development of seizures, while a lower myelin-content relates to a pathological condition that could be associated with epilepsy. Furthermore, reduced MWFs were also reported in three cases of pediatric epilepsy [7]. While these three cases presented very different clinical presentations, they all showed a decreased myelinwater content indicating that the involvement of myelin in epilepsy is robust. Recently, Drenthen et al. showed that the MWF in the frontal lobe of children with childhood absence epilepsy is reduced [8]. This further establishes the importance of myelin content in pediatric epilepsy.

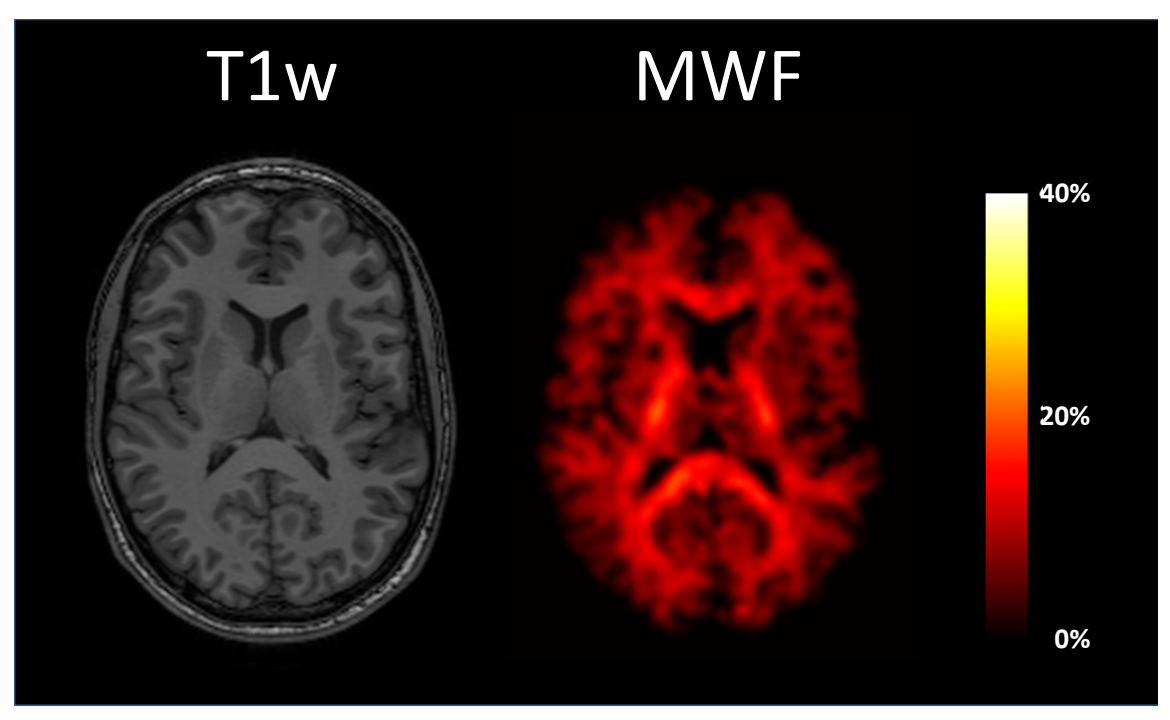

Figure 2.2: T1-weighted image and MWF map for an adult male subject (30 y). (Data from the authors' clinic)

\section{Discussion}

\section{Current findings}

To date, there are numerous preclinical and human studies that provide strong evidence that the myelin content might be related to epilepsy, epileptic seizures, and epileptogenesis. For example, in a wide range of preclinical epilepsy models abnormal myelination was reported. In almost all the models, epilepsy was associated with a reduced myelin content. From the current literature it cannot be confirmed whether the myelin abnormalities are an underlying cause of epilepsy, or a consequence of the excessive activity in epilepsy. However, administration of demyelination drugs was associated with epileptic seizures, possibly indicative of a relation between a reduced myelin content and seizures. Moreover, these 
provoked seizures were shown to be inhibited by certain anti-demyelination drugs. However, as of yet there is no evidence that administration of these drugs in human patients could inhibit seizures. Most of the imaging studies of myelin abnormalities in epilepsy have focused on qualitative MRI rather than quantitative measures of myelin content. While qualitative studies provide useful markers on white matter abnormalities, they fail to be specific the myelin content only. Therefore, quantitative and myelin-specific studies are important to further study white matter and myelin abnormalities in epilepsy. Three prior paediatric studies using myelin-specific quantitative measures have already showed that epilepsy is associated with myelin abnormalities [7], [8], [57]. However, additional myelin-specific imaging studies are warranted to further investigate the relation of the myelin content with epilepsy.

\section{Promising future methods}

\section{Magnetic resonance imaging}

The involvement of the myelin in epilepsy patients can be assessed in vivo using various methods of quantitative MRI. For example, magnetization transfer imaging can be used to detect abnormalities in the myelin content, via the magnetization transfer ratio (MTR) [79]. While the MTR is sensitive to the macromolecules in the myelin sheath, it is not specific enough to distinguish between myelin abnormalities and, for example, inflammatory processes [80]. The myelin sheath itself can also be imaged directly using ultra-short echo time (UTE) imaging [81]. While the transverse magnetization of the MR signal of the myelin sheath decays too quickly ( $10 \mu \mathrm{s}<\mathrm{T} 2<1 \mathrm{~ms}$ ) to be visible on conventional T2-weighted images, they can be visualized using UTE sequences. Although UTE time measurements are promising in the field of myelin quantification, they remain challenging on clinical MR systems due to hardware limitations [82]. These aforementioned techniques are yet to be applied in studies with epilepsy patients.

\section{PET}

Positron-emission tomography (PET) is a non-invasive nuclear imaging technique that can observe metabolic processes in vivo. After administration of a positron emitting radiotracer, pairs of gamma ray photons are detected by the scanner. The tracer is coupled to a chemical ligand that will be taken up or bind to the tissue of interest. PET can complement structural imaging (e.g. MRI) by providing specific biochemical information. Moreover, PET can detect biochemical changes before any macroscopic effects occur, enabling accurate assessment of demyelination. Using FDG as a (glucose uptake mimicking) radiotracer it has already been shown qualitatively that hypo(energy)metabolism was associated to delayed myelination 
[47]. Besides MRI, positron emission tomography (PET) has also been used to assess alterations in West syndrome. Using fluoro-deoxy-glucose (FDG), hypo(energy)metabolism was qualitatively assessed in cortical areas [47], [83], [84], which was found to be related to delayed myelination [47]. However, FDG is not be a specific tracer for myelin. To enable a more quantitative assessment, several more myelin-specific PET radiotracers have previously been developed.

For example, [11C]PIB, which is an 11-carbon tracer that was originally developed for amyloid imaging in Alzheimer's disease, has also been shown to be sensitive to myelin changes in preclinical models and humans [85], [86]. The [11C]PIB parametric maps were found to be reliable and reproducible [87]. Another promising tracer is [11C] N-methyl-4,4'diaminostilbene ([11C]MeDAS), which was shown to correlate well with myelin on histochemistry [88]. Alternatively, by targeting axonal potassium channels that become exposed upon demyelination, Brugarolas et al. used a radiolabelled derivative of a multiple sclerosis drug, 4-aminopyridine (4-AP), that binds to potassium channels as a quantification of demyelination [89]. They detected high levels of binding in demyelinated brain areas which decreased after remyelination.

While these methods provide promising perspectives for in vivo quantification of demyelination and remyelination, their added value in disorders with more subtle changes in myelin content compared to multiple sclerosis, such as epilepsy still need to be investigated. Moreover, although PET is non-invasive imaging technique, it does expose the patient to some degrees of ionizing radiation, complicating large clinical trials, especially for studies on children and longitudinal studies with repeated measurements. Furthermore, compared to MRI the spatial resolution of PET imaging is much lower.

\section{Conclusion}

In conclusion, this review shows that the currently available literature does not provide a complete view into the nature of myelin abnormalities in epilepsy. However, the reported literature is indicative of a relation between the pathophysiology of epilepsy and the myelin content. Therefore, future myelin-specific imaging studies might provide new avenues for understanding the underling mechanisms involved in epilepsy, epileptic seizures and the interaction with the vulnerable and maturing brain during childhood. 


\section{References}

[1] D. Kwon, A. Pfefferbaum, E. V Sullivan, and K. M. Pohl, "Regional growth trajectories of cortical myelination in adolescents and young adults: longitudinal validation and functional correlates," Brain Imaging Behav., 2018.

[2] P. Morell and R. Quarles, "Characteristic Composition of Myelin," in Basic Neurochemistry: Molecular, Cellular and Medical Aspects, 6th ed., G. Siegel, B. Agranoff, R. Albers, and P. Molinoff, Eds. Philadelphia, 1999.

[3] E. Kavroulakis et al., "Myelin content changes in probable Alzheimer's disease and mild cognitive impairment: Associations with age and severity of neuropsychiatric impairment," $J$. Magn. Reson. Imaging, vol. 47, no. 5, pp. 1359-1372, 2018.

[4] S. Kolind et al., "Clinical Brain and cord myelin water imaging: a progressive multiple sclerosis biomarker," NeuroImage Clin., vol. 9, pp. 574-580, 2015.

[5] D. C. Dean III et al., "Alterations of Myelin Content in Parkinson's Disease : A Cross-Sectional Neuroimaging Study," PLoS One, vol. 11, no. 10, p. e0163774, 2016.

[6] D. J. M. Lang et al., "Clinical 48 echo T2 myelin imaging of white matter in first-episode schizophrenia: Evidence for aberrant myelination," NeuroImage Clin., vol. 6, pp. 408-414, 2014.

[7] H. S. Spader et al., "Advances in myelin imaging with potential clinical application to pediatric imaging," Neurosurg. Focus, vol. 34, no. 4, p. E9, Apr. 2013.

[8] G. S. Drenthen et al., "Lower myelin-water content of the frontal lobe in childhood absence epilepsy," Epilepsia, vol. 60, no. 8, pp. 1689-1696, 2019.

[9] K. M. Fiest et al., "Prevalence and incidence of epilepsy A systematic review and meta-analysis of international studies," Neurology, vol. 88, pp. 296-303, 2017.

[10] R. S. Fisher et al., "A practical clinical definition of epilepsy," Epilepsia, vol. 55, no. 4, pp. 475-482, 2014.

[11] J. H. Jackson, "The Lumleian Lectures on Convulsive Seizures," BMJ, vol. 1, no. 1528, pp. 821827, Apr. 1890.

[12] W. M. Otte, P. Van Eijsden, J. W. Sander, J. S. Duncan, R. M. Dijkhuizen, and K. P. J. Braun, “A meta-analysis of white matter changes in temporal lobe epilepsy as studied with diffusion tensor imaging," Epilepsia, vol. 53, no. 4, pp. 659-667, 2012.

[13] I. E. Scheffer et al., "ILAE classification of the epilepsies: Position paper of the ILAE Commission for Classification and Terminology," Epilepsia, vol. 58, no. 4, pp. 512-521, 2017.

[14] E. B. Bromfield, J. E. Cavazos, and J. I. Sirven, Eds., An Introduction to Epilepsy. West Hartford (CT): American Epilepsy Society, 2006.

[15] T. M. Hyde and D. R. Weinberger, "Seizures and Schizophrenia," Schizophr. Bull., vol. 23, no. 4, pp. 611-622, 1997.

[16] K. Gruntz et al., "Parkinson Disease and the Risk of Epileptic Seizures," Ann. Neurol., vol. 83, pp. 363-374, 2018.

[17] D. Pandis and N. Scarmeas, "Seizures in Alzheimer Disease: Clinical and Epidemiological Data," Epilepsy Curr., vol. 12, no. 5, pp. 184-187, 2012.

[18] A. S. Lapato, J. I. Szu, J. P. C. Hasselmann, A. J. Khalaj, D. K. Binder, and S. K. Tiwari-Woodruff, "Chronic demyelination-induced seizures," Neuroscience, vol. 346, pp. 409-422, 2017.

[19] C. M. Poser and V. V. Brinar, "Epilepsy and multiple sclerosis," Epilepsy Behav., vol. 4, no. 1, pp. 6-12, 2003.

[20] G. Slinger, M. R. T. Sinke, K. P. J. Braun, and W. M. Otte, "White matter abnormalities at a regional and voxel level in focal and generalized epilepsy: A systematic review and metaanalysis," NeuroImage Clin., vol. 12, pp. 902-909, 2016.

[21] T. Inui et al., "The spontaneously epileptic rat (SER), a zitter*tremor double mutant rat: histopathological findings in the central nervous system," Brain Res., vol. 517, no. 1-2, pp. 123$133,1990$. 
[22] K. Hoffmann, M. Lindner, I. Gröticke, M. Stangel, and W. Löscher, "Epileptic seizures and hippocampal damage after cuprizone-induced demyelination in C57BL / 6 mice," Exp. Neurol., vol. 210, pp. 308-321, 2008.

[23] L. C. Taylor, K. Puranam, W. Gilmore, J. P. Ting, and G. K. Matsushima, “Neurobiology of Disease $17 \beta$-estradiol protects male mice from cuprizone-induced demyelination and oligodendrocyte loss," Neurobiol. Dis., vol. 39, no. 2, pp. 127-137, 2010.

[24] Y. You et al., "Myelin damage of hippocampus and cerebral cortex in rat pentylenetetrazol model," Brain Res., vol. 1381, pp. 208-216, 2011.

[25] Y. You et al., "Glatiramer acetate, an anti-demyelination drug, reduced rats' epileptic seizures induced by pentylenetetrazol via protection of myelin sheath," Eur. J. Pharm. Sci., vol. 49, no. 3, pp. 366-370, 2013.

[26] M. Gol, D. Ghorbanian, S. Hassanzadeh, and M. Javan, “Fingolimod enhances myelin repair of hippocampus in pentylenetetrazol-induced kindling model," Eur. J. Pharm. Sci., vol. 96, pp. 72 83, 2017.

[27] L. Ma et al., "Quetiapine attenuates cognitive impairment and decreases seizure susceptibility possibly through promoting myelin development in a rat model of malformations of cortical development," Brain Res., vol. 1622, pp. 443-451, 2015.

[28] K. J. Hopkins, G. Wang, and L. C. Schmued, "Temporal progression of kainic acid induced neuronal and myelin degeneration in the rat forebrain," Brain Res., vol. 864, pp. 69-80, 2000.

[29] A. Sierra, T. Laitinen, K. Lehtimaki, L. Rieppo, A. Pitkanen, and O. Grohn, "Diffusion tensor MRI with tract-based spatial statistics and histology reveals undiscovered lesioned areas in kainate model of epilepsy in rat," Brain Struct Funct, vol. 216, pp. 123-135, 2011.

[30] W. M. Otte, P. Bielefeld, R. M. Dijkhuizen, and K. P. J. Braun, “Focal neocortical epilepsy affects hippocampal volume, shape, and structural integrity: A longitudinal MRI and immunohistochemistry study in a rat model," Epilepsia, vol. 53, no. 7, pp. 1264-1273, 2012.

[31] Y. Ye et al., "Altered hippocampal myelinated fiber integrity in a lithium-pilocarpine model of temporal lobe epilepsy: A histopathological and stereological investigation," Brain Res., vol. 1522, pp. 76-87, 2013.

[32] Y. Luo et al., "Alterations in hippocampal myelin and oligodendrocyte precursor cells during epileptogenesis," Brain Res., vol. 1627, pp. 154-164, 2015.

[33] J. M. Barry, C. Tian, A. Spinella, M. Page, and G. L. Holmes, "Epilepsy \& Behavior Spatial cognition following early-life seizures in rats: Performance deficits are dependent on task demands," Epilepsy Behav., vol. 60, pp. 1-6, 2016.

[34] A. M. Sherafat et al., "Kindling-induced learning deficiency and possible cellular and molecular involved mechanisms," Neurol. Sci., no. 34, pp. 883-890, 2013.

[35] P. Sharma et al., "Differences in white matter structure between seizure prone (FAST) and seizure resistant (SLOW) rat strains," Neurobiol. Dis., vol. 104, pp. 33-40, 2017.

[36] P. Sharma, K. L. Powell, M. E. Wlodek, T. J. O. Brien, and K. L. Gilby, “Delayed myelination and neurodevelopment in male seizure-prone versus seizure-resistant rats," no. January, pp. 753764, 2018.

[37] Ö. Daniş et al., "Changes in intracellular protein expression in cortex, thalamus and hippocampus in a genetic rat model of absence epilepsy," Brain Res. Bull., vol. 84, no. 6, pp. 381388, 2011.

[38] O. Ozdogmus, S. Cavdar, Y. Ersoy, F. Ercan, and U. Ibrahim, "A preliminary study, using electron and light-microscopic methods, of axon numbers in the fornix in autopsies of patients with temporal lobe epilepsy," Anat Sci Int, vol. 84, pp. 2-6, 2009.

[39] T. Wang et al., "Human Ermin (hErmin), a new oligodendrocyte-specific cytoskeletal protein related to epileptic seizure," Brain Res., vol. 1367, pp. 77-84, 2010.

[40] X. Hu et al., "The relationship between the occurrence of intractable epilepsy with glial cells and myelin sheath - an experimental study.," Eur. Rev. Med. Pharmacol. Sci., vol. 20, no. 21, pp. 45164524, 2016. 
[41] P. Van Eijsden et al., "In vivo diffusion tensor imaging and ex vivo histologic characterization of white matter pathology in a post-status epilepticus model of temporal lobe epilepsy," Epilepsia, vol. 52, no. 4, pp. 841-845, 2011.

[42] A. Sierra and T. Laitinen, "Diffusion tensor imaging of hippocampal network plasticity," Brain Struct Funct, vol. 220, pp. 781-801, 2015.

[43] R. A. Salo, T. Miettinen, T. Laitinen, O. Gröhn, and A. Sierra, "Diffusion tensor MRI shows progressive changes in the hippocampus and dentate gyrus after status epilepticus in rat histological validation with Fourier-based analysis," Neuroimage, vol. 152, pp. 221-236, 2017.

[44] K. Watanabe, T. Haga, T. Negoro, K. Aso, and N. Maeda, "Focal Spasms in Clusters, Focal Delayed Myelination, and Hypsarrhythmia: Unusual Variant of West Syndrome," Pediatr. Drugs, vol. 11, pp. 47-49, 1994.

[45] C. Schropp et al., "Delayed Myelination in Children with West Syndrome: An MRI-Study," Neuropediatrics, vol. 25, no. 3, pp. 116-120, 1994.

[46] M. Staudt et al., "MRI assessment of myelination: an age standardization," Pediatr Radiol, vol. 24, pp. 122-127, 1994.

[47] J. Natsume et al., "Cortical Hypometabolism and Delayed Myelination in West Syndrome," Epilepsia, vol. 37, no. 12, pp. 1180-1184, 1996.

[48] J. Muroi et al., "An MRI study of the myelination pattern in West syndrome," Brain Dev., vol. 7604, no. 81, pp. 179-184, 1996.

[49] N. Aydinli, M. Caliskan, M. Ozmen, and E. Tonguc, "Neuroradiologic Aspects of West Syndrome," Pediatr. Neurol., vol. 8994, no. 98, pp. 211-216, 1998.

[50] T. Takano, A. Hayashi, T. Sokoda, and C. Sawai, "Delayed Myelination at the Onset of Cryptogenic West Syndrome," Pediatr. Neurol., vol. 37, no. 6, pp. 417-420, 2007.

[51] J. Tohyama, N. Akasaka, H. Osaka, and Y. Maegaki, "Early onset West syndrome with cerebral hypomyelination and reduced cerebral white matter," Brain Dev., vol. 30, pp. 349-355, 2008.

[52] L. Bartolini et al., "Temporal lobe epilepsy and focal cortical dysplasia in children: A tip to find the abnormality," Epilepsia, vol. 58, no. 1, pp. 113-122, 2017.

[53] F. Deodato, M. Sabatelli, E. Ricci, E. Mercuri, F. Muntoni, and C. Sewry, "Hypermyelinating neuropathy, mental retardation and epilepsy in a case of merosin deficiency," Neuromuscul. Disord., vol. 12, pp. 392-398, 2002.

[54] G. Goldsberry, D. Mitra, D. Macdonald, and Z. Patay, "Accelerated myelination with motor system involvement in a neonate with immediate postnatal onset of seizures and hemimegalencephaly," Epilepsy Behav., vol. 22, no. 2, pp. 391-394, 2011.

[55] C. M. Eltze, W. K. Chong, S. Bhate, B. Harding, B. G. R. Neville, and J. H. Cross, “Taylor-type Focal Cortical Dysplasia in Infants: Some MRI Lesions Almost Disappear with Maturation of Myelination," Epilepsia, vol. 46, no. 12, pp. 1988-1992, 2005.

[56] B. C. Bernhardt et al., "Preferential susceptibility of limbic cortices to microstructural damage in temporal lobe epilepsy: A quantitative T1 mapping study," Neuroimage, vol. 182, pp. 294-303, 2018.

[57] K. Moldovan et al., "Myelin water fraction changes in febrile seizures," Clin. Neurol. Neurosurg., vol. 175, pp. 61-67, 2018.

[58] C. Ji-qun, K. Ishihara, T. Nagayama, T. Serikawa, and M. Sasa, "Long-lasting Antiepileptic Effects of Levetiracetam against Epileptic Seizures in the Spontaneously Epileptic Rat (SER): Differentiation of Levetiracetam from Conventional Antiepileptic Drugs," Epilepsia, vol. 46, no. 9, pp. 1362-1370, 2005.

[59] H. Yan, C. Ji-qun, K. Ishihara, T. Nagayama, T. Serikawa, and M. Sasa, "Separation of Antiepileptogenic and Antiseizure Effects of Levetiracetam in the Spontaneously Epileptic Rat (SER)," Epilepsia, vol. 46, no. 8, pp. 1170-1177, 2005.

[60] Ø. Torkildsen, B. La, M. K-m, and B. L. The, “The cuprizone model for demyelination," Acta Neurol Scand, vol. 117, pp. 72-76, 2008. 
[61] M. Virgili, P. Migani, A. Contestabile, and O. Barnabei, "Protection from kainic acid neuropathological syndrome by NMDA receptor antagonists: effect of MK-801 and CGP 39551 on neurotransmitter and glial markers.," Neuropharmacology, vol. 31, no. 5, pp. 469-474, May 1992.

[62] J. G. Jefferys, C. Borck, and J. Mellanby, “Chronic focal epilepsy induced by intracerebral tetanus toxin," Ital. J. Neurol. Sci., vol. 16, pp. 27-32, 1995.

[63] D. McCormic and D. Contreras, "On the cellular and network bases of epileptic seizures," Аnnu. Rev. Physiol., vol. 6, no. 1, pp. 298-307, 2001.

[64] C. Shepherd et al., "A quantitative study of white matter hypomyelination and oligodendroglial maturation in focal cortical dysplasia type II," Epilepsia, vol. 54, no. 5, pp. 898-908, 2013.

[65] D. Taylor, M. Falconer, C. Bruton, and J. Corsellis, "Focal dysplasia of the cerebral cortex in epilepsy," J Neurol Neurosurg Psychiatry, vol. 34, pp. 369-387, 1971.

[66] T. Scholl et al., "Impaired oligodendroglial turnover is associated with myelin pathology in focal cortical dysplasia and tuberous sclerosis complex," Brain Pathol., vol. 27, no. 6, pp. 770-780, 2017.

[67] F. Deleo, M. Thom, L. Concha, A. Bernasconi, B. C. Bernhardt, and N. Bernasconi, "Histological and MRI markers of white matter damage in focal epilepsy," Epilepsy Res., vol. 140, pp. 29-38, 2018.

[68] P. Pavone, P. Striano, R. Falsaperla, L. Pavone, and M. Ruggieri, "Infantile spasms syndrome, West syndrome and related phenotypes: What we know in 2013," Brain Dev., vol. 36, no. 9, pp. 739-751, 2014.

[69] H. Ozawa, T. Hashimoto, T. Endo, and T. Kato, "West Syndrome With Periventricular Leukomalacia: A Morphometric MRI Study," Pediatr. Neurol., vol. 19, no. 5, pp. 358-363, 1998.

[70] R. Sankar, J. G. Curran, J. W. Kevill, P. J. Rintahaka, D. A. Shewmon, and H. V Vinters, "Microscopic Cortical Dysplasia in Infantile Spasms: Evolution of White Matter Abnormalities," Am J Neuroradiol, vol. 16, pp. 1265-1272, 1995.

[71] J. L. Holtrop, T. M. Loucks, J. J. Sosnoff, and B. P. Sutton, "Investigating Age-related changes in fine motor control across different effectors and the impact of white matter integrity," Neuroimage, vol. 96, pp. 81-87, Aug. 2014.

[72] P. S. Buckmaster, "Mossy Fiber Sprouting in the Dentate Gyrus," in Jasper's Basic Machanisms of the Elipepsies, 4th ed., J. L. Noebels, M. Avoli, M. A. Rogawski, R. W. Olson, and A. V. DelgadoEscueta, Eds. Bethesda (MD): National Center for Biotechnology Information (US), 2012.

[73] C. Laule et al., "Magnetic resonance imaging of myelin," Neurotherapeutics, vol. 4, no. 3, pp. 460484, Jul. 2007.

[74] L. Concha, D. J. Livy, C. Beaulieu, B. M. Wheatley, and D. W. Gross, "In Vivo Diffusion Tensor Imaging and Histopathology of the Fimbria-Fornix in Temporal Lobe Epilepsy," Neurobiol. Dis., vol. 30, no. 3, pp. 996-1002, 2010.

[75] G. Lockwood-estrin et al., "Correlating 3T MRI and histopathology in patients undergoing epilepsy surgery," J. Neurosci. Methods, vol. 205, no. 1, pp. 182-189, 2012.

[76] C. Reeves et al., "Combined Ex Vivo 9.4T MRI and Quantitative Histopathological Study in Normal and Pathological Neocortical Resections in Focal Epilepsy," Brain Pathol., vol. 26, pp. 319-333, 2015.

[77] S. H. Koenig, R. D. Brown III, M. Spiller, and N. Lundbom, "Relaxometry of brain: Why white matter appears bright in MRI," Magn. Reson. Med., vol. 14, no. 3, pp. 482-495, 1990.

[78] E. Alonso-Ortiz, I. R. Levesque, and G. B. Pike, "MRI-based myelin water imaging: A technical review," Magn. Reson. Med., vol. 73, no. 1, pp. 70-81, Jan. 2015.

[79] M. A. van Buchem et al., "Global estimation of myelination in the developing brain on the basis of magnetization transfer imaging: a preliminary study," Am. J. Neuroradiol., vol. 22, no. 4, pp. 762-6, Apr. 2001.

[80] I. M. Vavasour, C. Laule, D. K. B. Li, A. L. Traboulsee, and A. L. Mackay, "Is the Magnetization Transfer Ratio a Marker for Myelin in Multiple Sclerosis?," J. Magn. Reson. Imaging, vol. 33, pp. 713-718, 2011. 
[81] V. Sheth et al., "Magnetic resonance imaging of myelin using ultrashort Echo time (UTE) pulse sequences: Phantom, specimen, volunteer and multiple sclerosis patient studies," Neuroimage, vol. 136, pp. 37-44, Aug. 2016.

[82] A. C. Seifert, C. Li, M. J. Wilhelm, S. L. Wehrli, and F. W. Wehrli, “Towards quantification of myelin by solid-state MRI of the lipid matrix protons," Neuroimage, vol. 163, pp. 358-367, Dec. 2017.

[83] N. Maeda et al., "Evolutional changes of cortical hypometabolism in West's syndrome," Lancet, vol. 343, pp. 1620-1623, 1994.

[84] N. Maeda et al., "Transient Focal Cortical Hypometabolism in Idiopathic West Syndrome," Pediatr. Neurol., vol. 9, no. 6, pp. 430-434, 1993.

[85] E. Grecchi, M. Veronese, B. Bodini, M. Battaglini, B. Stankoff, and F. E. Turkheimer, "Multimodal partial volume correction: Application to [11C] PIB PET/MRI myelin imaging in multiple sclerosis," J. Cereb. Blood Flow Metab., vol. 37, no. 12, pp. 3803-3817, 2017.

[86] B. Stankoff et al., "Myelin by Positron Emission Tomography in Multiple Sclerosis Using [Methyl-11C]-2-(40-Methylaminophenyl)-6-Hydroxybenzothiazole," Ann. Neurol., vol. 69, no. 4, pp. 673-680, 2011.

[87] M. Veronese, B. Bodini, D. García-lorenzo, M. Battaglini, S. Bongarzone, and C. Comtat, "Quantification of [11C] PIB PET for imaging myelin in the human brain: a test-retest reproducibility study in high-resolution research tomography," J. Cereb. Blood Flow Metab., vol. 35, pp. 1771-1782, 2015.

[88] D. de Paula Faria, E. F. J. De Vries, J. W. A. Sijbesma, R. A. J. O. Dierckx, C. A. Buchpiguel, and S. Copray, "PET imaging of demyelination and remyelination in the cuprizone mouse model for multiple sclerosis: A comparison between [11C] CIC and [11C] MeDAS," Neuroimage, vol. 87, pp. 395-402, 2014.

[89] P. Brugarolas et al., "Development of a PET radioligand for potassium channels to image CNS demyelination," Sci. Rep., vol. 8, no. 1, p. 607, 2018. 



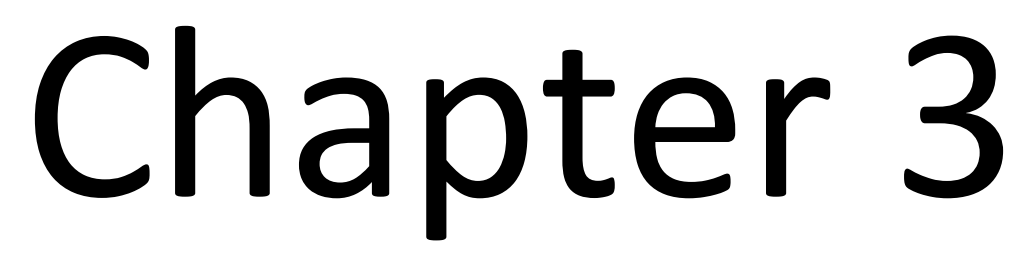

\section{A new analysis approach for $\mathrm{T} 2$ relaxometry myelin-water quantification: Orthogonal Matching Pursuit}

Gerhard S. Drenthen, Walter H. Backes, Albert P. Aldenkamp, Giel J. Op 't Veld, Jacobus F.A. Jansen

Magn. Reson. Med. 2019; 81(5):3292-3303. DOI: 10.1002/mrm.27600 
CHAPTER 3 


\section{Abstract}

In vivo myelin quantification can provide valuable non-invasive information on neuronal maturation and development, as well as insights into neurological disorders. Multiexponential analysis of multi-echo T2 relaxation is a powerful and widely applied method for the quantification of the myelin-water fraction (MWF). In recent literature, the MWF is most commonly estimated using a regularized non-negative least squares (NNLS) algorithm.

The Orthogonal Matching Pursuit (OMP) algorithm is proposed as an alternative method for the estimation of the MWF. The OMP is a greedy sparse reconstruction algorithm with a low computation complexity. For validation, both methods are compared to a ground truth using numerical simulations and a phantom model using comparable computation times. The numerical simulations were used to measure the theoretical errors, as well as the effects of varying the signal-to-noise ratio, strength of the regularization and resolution of the basis set. Additionally, a phantom model was used to estimate the performance of the two methods while including errors occurring due to the MR measurement. Lastly, four healthy subjects were scanned to evaluate the in vivo performance.

The results in simulations and phantoms demonstrate that the MWFs determined with the OMP are 1.7 times more accurate as compared to the NNLS, with a comparable precision. The remaining bias of the MWF is shown to be related to the regularization of the NNLS algorithm and the Rician noise present in magnitude MR images. The OMP algorithm provides a more accurate alternative for $\mathrm{T} 2$ relaxometry myelin-water quantification. 


\section{Introduction}

MRI is a helpful technique to detect abnormalities in the cerebral white matter. For instance, T2-weighted imaging techniques grant excellent contrast to identify white matter abnormalities, and diffusion weighted MRI (DWI) provides information on the integrity of the white matter tracts [1]. While these methods are invaluable in the ongoing research and clinical diagnosis of white matter disorders, they fail to provide more specific information on one of the most characteristic components of the white matter, the myelin.

Myelin is a layered, fatty substance wrapped around the axons that is comprised of lipids and proteins. The myelin acts as an electrical insulator, accelerating the transport of electrical signals along the axons. In addition to the dry mass the myelin volume is roughly made up of $40 \%$ water, which is trapped between the lipid bilayers of the myelin sheath [2]. Myelin is vital to healthy neuronal development, and can therefore provide valuable information regarding neuronal maturation and development, as well as insights into disintegration as part of several neurological disorders [3]. Therefore, MRI techniques that yield specific information on the myelin content can provide information on white matter that complements structural T2-weighted and DWI techniques.

White matter is relatively bright on T1-weighted images which is related to myelinbound cholesterol, while the T2-weighted contrast of white matter is low due to motionrestricted protons in the myelin-water. Previously, both the T1- and T2-weighted images were combined to enhance myelin contrast by calculating the ratio of T1- and T2-weighted image intensities [4]. Despite the enhanced myelin contrast, the T1/T2 ratio fails to provide information that is specific to the myelin. Besides conventional imaging methods, DWI is often used to study the white matter. However, while DWI measures can provide information on changes in myelin, they are not suitable for absolute quantification of myelin content [5]. Another technique which is used to detect abnormalities in myelin content is magnetization transfer imaging, via the magnetization transfer ratio [6]. While the magnetization transfer ratio is sensitive to the interaction of water molecules with the macromolecules in the myelin sheath, it is not specific enough to distinguish between myelin abnormities and, for example, inflammatory processes [7]. Contrary to these indirect measures of myelin, ultra-short echo time imaging can be used to image the myelin sheath directly [8]. Though the MR signal of the myelin sheath dephases too quickly $(10 \mu \mathrm{s}<\mathrm{T} 2<1 \mathrm{~ms})$ to be visible on conventional T2weighted images, it can be visualized using ultra-short echo time sequences. Although ultrashort echo time measurements are promising in the field of myelin quantification, they remain challenging on clinical MR systems due to hardware limitations [9].

Since water is a significant part of the myelin volume, many studies have focused on the quantification of myelin-water as an indirect measure of myelin content. Myelin-water can be quantified using either the multicomponent driven equilibrium single pulse observation of T1 and T2 (mcDESPOT) [10] or the analysis of T2 relaxometry. In the current study, T2 relaxometry is used to quantify the myelin-water. The rationale for using $\mathrm{T} 2$ relaxometry to 
quantify myelin content is that trapped and motion-restricted water particles between the lipid layers of the myelin sheath have a faster T2-weighted signal decay $(10 \mathrm{~ms}<\mathrm{T} 2<40 \mathrm{~ms})$ compared to more freely moving water in the intra- and extracellular spaces $(80 \mathrm{~ms}<\mathrm{T} 2<100$ $\mathrm{ms}$ ) and CSF (T2 > $2 \mathrm{~s}$ ) [11]. Due to these differences in T2 relaxation time, MR imaging can provide surrogate markers of myelin content via the myelin-water, whereas the T2 relaxation profile of a single voxel is a superposition of myelin-water, intra-extra cellular water, and CSF components. The signal attributed to each of these components can then be determined by separating the measured signal into its constituting relaxation components. From the resulting relaxation components, the fraction of myelin-water signal to the total water signal, the myelin-water fraction (MWF), can be determined. Bi-exponential fitting methods are typically unsuitable for the extraction of the constituting components, as they are unstable and, furthermore, assume that only two components are present, which is not guaranteed. Alternatively, the constituting components can be extracted using multi-exponential analysis. In literature, the multi-exponential analysis is most commonly performed with the nonnegative Least Squares (NNLS) algorithm [12]-[16]. Opposed to bi-exponential fitting methods, the NNLS algorithm makes no assumptions on the number of underlying components and does not require an estimation of the solution to start the optimization algorithm. Instead, the NNLS reconstructs the signal from a predefined overcomplete basis set of $\mathrm{T} 2$ relaxation decay curves, the so-called dictionary.

In the current study, we propose the orthogonal matching pursuit (OMP) algorithm [17] as an alternative to estimate the MWF using T2 relaxation MRI. Common applications of the $\mathrm{OMP}$ are wavelet decomposition, denoising of images, and reconstruction of images or signals (e.g. NMR spectroscopy spectra) [18]-[21]. Similar to the NNLS, the OMP uses an overcomplete dictionary to estimate the corresponding weights of each element from the dictionary. However, in contrast to the NNLS, the OMP is a greedy algorithm which aims to model as much of the current residuals in each step, rather than working towards the global optimum [22].

In the following we compare the performance of the OMP and NNLS algorithms on estimating the MWF using T2 relaxometry. To investigate the theoretical errors for varying SNR of both methods, numerical simulations are used. A phantom model is employed to assess errors related to the MR acquisition, and lastly, the two methods are compared in healthy volunteers. 


\section{Theory}

\section{Non-negative least squares}

Introduced by Lawson and Hanson [23], and extensively investigated and applied to T2 relaxation data by Whittall and MacKay [16] for the first time, the NNLS uses a predefined basis set $\mathbf{A}$ with $M$ elements of discrete $T_{2 j}$ relaxation decay curves to characterize the measured signal $y_{i}$ as

$$
y_{i}=\sum_{j=1}^{M} s_{j} \exp \left(-t_{i} / T_{2 j}\right)=\sum_{j=1}^{M} \mathbf{A}_{i j} s_{j}, \quad i=1,2, \ldots, N
$$

where $s_{j}$ is the amplitude corresponding to the $T_{2 j}$ relaxation time, $t_{i}$ is the measurement time of data point $i$ and $N$ represents the total number of data points. Now, the NNLS problem can be written as,

$$
\chi_{\min }^{2}=\min _{s \geq 0}\left(\sum_{i=1}^{N}\left|\sum_{j=1}^{M} \mathbf{A}_{i j} s_{j}-y_{i}\right|^{2}\right)
$$

where $\chi_{\min }^{2}$ represents the misfit that is minimized by the NNLS algorithm. Typically, in T2 relaxation analysis the number of data points is smaller compared to the elements in the basis set $(\mathrm{N}<\mathrm{M})$ making the NNLS an ill-posed problem. To provide a more stable solution, a regularized version of the NNLS is used, adding a smoothing constraint,

$$
\chi_{\mathrm{reg}}^{2}=\min _{s \geq 0}\left(\sum_{i=1}^{N}\left|\sum_{j=1}^{M} \mathbf{A}_{i j} s_{j}-y_{i}\right|^{2}+\mu \sum_{j=1}^{M}\left|s_{j+2}-2 s_{j+1}+s_{j}\right|^{2}\right),
$$

where $\mu$ is the regularization parameter and $\chi_{\mathrm{reg}}^{2}$ is the regularized misfit. As the regularization term represents a numerical derivative, a larger $\mu$ results in a smoother amplitude distribution $(s)$ at the cost of a larger misfit $\left(\chi_{\text {reg }}^{2}\right)$. The NNLS is a convex algorithm with a unique solution. However, the regularized NNLS has a high computational complexity, limiting the number of elements in the basis set. Typically, 120 logarithmically spaced elements ranging from 15 to $2000 \mathrm{~ms}$ are used [24]-[27]. 


\section{Orthogonal matching pursuit}

The OMP algorithm, introduced by Davis, Mallat and Zhang [17], builds a sparse representation to estimate the measured signal $y$ by iteratively selecting that element from a predefined basis set $\mathbf{A}$ which correlates most with the current residual (i.e. greedy optimization) [20]. To initialize the algorithm, the measured signal serves as the first (artificial) residual. To ensure positive weights, Bruckstein et al. proposed a non-negative implementation of the OMP by solving an unconstrained non-negative least squares problem for a subset of $\mathbf{A}$ with the current selection of $K$ elements, $\widehat{\mathbf{A}}_{K}$, in each iteration [28],

$$
\chi_{\min }^{2}=\min _{s \geq 0}\left(\sum_{i=1}^{N}\left|\sum_{j=1}^{K} \widehat{\mathbf{A}}_{K i j} s_{j}-y_{i}\right|^{2}\right) .
$$

Note that the weights of previously selected elements can become zero, which means that not all of the selected elements are necessarily represented in the final signal representation. From this point on, we will refer to the aforementioned non-negative implementation of the OMP simply as OMP. The OMP iterates until one of two stopping criteria is met: (i) either a user defined number of elements $(n)$ is selected from $\mathbf{A}$, or (ii) there is no new element from the dictionary that positively correlates with the last residual. Thus, the OMP algorithm combines the low computational complexity of a greedy algorithm with the stability of a least-squares solver. The OMP algorithm is shown in pseudo-code (Algorithm 3.1) [29]. Greedy algorithms such as the OMP have a low computational complexity, thus allowing a higher resolution (e.g. 1000 or more basis set elements).

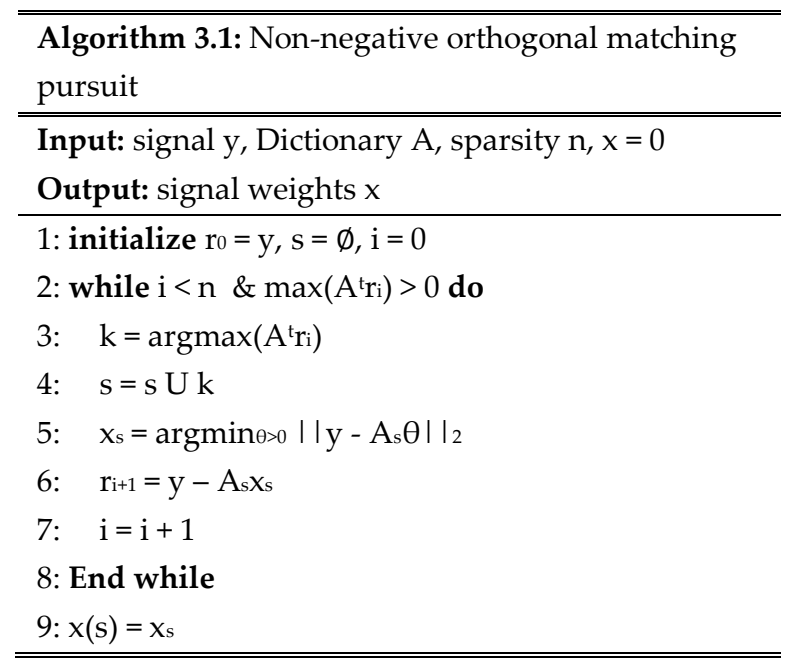




\section{Myelin-water fraction}

For both methods, the MWF is subsequently defined as the amplitude fraction of the $T_{2 j}$ relaxation elements associated with myelin-water to all $T_{2 j}$ relaxation water elements, where the myelin-water component is expected to have a T2 relaxation time in the range of $15-40 \mathrm{~ms}$ at $3 \mathrm{~T}[14]$.

\section{Methods}

\section{Numerical simulations}

To estimate the numerical errors of the NNLS and OMP algorithms, multi-exponential relaxation curves were computationally synthesized. To also include effects of stimulated echoes caused by B1 inhomogeneities, the extended phase graph (EPG) algorithm [30] was used to synthesize relaxation data with an imperfect refocusing flip angle of $150^{\circ}$ [31], [32] and 32 echo times with an echo spacing of $12 \mathrm{~ms}$ (range 12-384 ms). The relaxation rates used for the water components in the simulations were $\mathrm{T} 1=1000 \mathrm{~ms}$, T2long $=100 \mathrm{~ms}$ and $\mathrm{T} 2$ myelin $=$ $30 \mathrm{~ms}$, corresponding to healthy white matter tissue. Furthermore, additional data was synthesized simulating a white matter lesion using T1 $=600 \mathrm{~ms}$, T2 1 esion $=200 \mathrm{~ms}$ and T2 myelin $=$ $30 \mathrm{~ms}$ [33], [34]. However, the shape of the underlying in vivo T2 distribution is unknown, and might behave more like a continuous distribution instead of a discrete distribution. Therefore, to study the potential effects of the underlying distribution on the MWF estimation of both algorithms, also a continuous Gaussian distribution consisting of two pools (mean \pm SD, T2long: $100 \pm 10 \mathrm{~ms}$, T2lesion: $200 \pm 20 \mathrm{~ms}, \mathrm{~T} 2$ myelin: $30 \pm 3 \mathrm{~ms}$ ) was reconstructed [35], [36].

To estimate the effects of myelin-water content and SNR on the analysis, 1000 Rician noise realizations were calculated for varying MWF (range 0-30\%, with $1 \%$ increment) and SNR (100, 200 and 350). Additionally, the effects of a smaller echo spacing of 8 ms (range 8$256 \mathrm{~ms}$ ) and a lower T2myelin peak time of $15 \mathrm{~ms}$ were also assessed for an SNR of 200. Rician noise was simulated as the magnitude of the signal with additive complex-valued Gaussian noise, and SNR was defined as the signal at the first echo (TE = $12 \mathrm{~ms}$ ) divided by the standard deviation of the added complex-valued Gaussian noise. Rician distributed noise is nearly Gaussian for high SNR values (>3), however we still need to take the Rician distributed noise into account as the SNR decreases exponentially for increasing TE.

\section{Phantom model}

To estimate errors in MWF estimation due to the signal acquisition, two vials with manganese (II) chloride solutions were prepared with concentrations of $.07 \mathrm{mM}$ and $.25 \mathrm{mM}$, resulting in mono-exponential $\mathrm{T} 2$ relaxation curves with $\mathrm{T} 2$ long $\approx 110 \mathrm{~ms}$ and $\mathrm{T} 2$ myelin $\approx 30 \mathrm{~ms}$ and $\mathrm{T} 1$ 
relaxation values of $\mathrm{T} 1_{\text {long }} \approx 1300$ and $\mathrm{T} 1_{\text {myelin }} \approx 500$, respectively. Ideally, the $\mathrm{T} 1$ myelin should be closer to $1000 \mathrm{~ms}$, however it is still sufficiently long (T1 > T2) to have no substantial effect in the subsequent EPG analyses [37]. Multi-exponential decays were synthesized by summing two randomly chosen voxels from each vial and weighing them such that a varying MWF (range $0-30 \%$, with $1 \%$ increment) is obtained. This process was repeated 1000 times. SNR of the phantom model was estimated as the mean signal of a ROI located in the vials at the first echo ( $\mathrm{TE}=12 \mathrm{~ms}$ ) divided by the standard deviation measured in four ROIs in the air placed in each corner of the image. As the measured noise is Rician distributed, a correction is applied such that, $\frac{\sigma_{R i c e}}{\sqrt{2-\pi / 2}}=\sigma_{\mathrm{Gauss}}$ is used [38].

\section{In vivo data}

Four healthy adult volunteers (age, range 26-30 years, 3 males) were scanned, and a single transverse slice MWF map was determined for each individual using both quantification methods. Additionally, a fifth healthy adult volunteer (age 29, female) was scanned using a 3D gradient and spin-echo (GRASE) sequence. The SNR of the in vivo data was determined as the mean signal in the genu and splenium of the corpus callosum divided by the standard deviation measured in four ROIs in the air placed in each corner of the image. All volunteers gave written permission and the study was conducted with the approval of the institutional review board.

\section{MRI acquisition}

The vials and healthy volunteers were scanned on a 3 Tesla unit (Philips Achieva, Best, the Netherlands) with an 32-element head coil using a single transverse slice multi-spin-echo (MSE) sequence $(\mathrm{TR}=3000 \mathrm{~ms}, 32$ echoes with the shortest possible echo spacing for this sequence of $12 \mathrm{~ms}$, range 12-384 ms, FOV $240 \times 198 \times 4$ mm, acquisition matrix size $160 \times 132$, voxel size $1.5 \times 1.5 \times 4 \mathrm{~mm}$, and 2 signal averages) [39]. In vivo, the slice is positioned through the genu and splenium of the corpus callosum and angulated parallel to the inferior edges of the corpus callosum. A single healthy volunteer was scanned using a 3D GRASE sequence (TR = $611 \mathrm{~ms}, 32$ echoes with echo spacing of $13 \mathrm{~ms}$, range 13-416 ms, FOV $240 \times 198 \times 128$ $\mathrm{mm}$, acquisition matrix size $160 \times 132 \times 32$, voxel size $1.5 \times 1.5 \times 4 \mathrm{~mm}$, EPI factor $=3$, Turbo factor $=32$, SENSE $=2$ ). For anatomical reference, T1-weighted 3D turbo field echo images were acquired for all healthy volunteers $\left(\mathrm{TR}=8.2 \mathrm{~ms}, \mathrm{TE}=3.7 \mathrm{~ms}, \mathrm{TI}=1010 \mathrm{~ms}, \mathrm{FA}=8^{\circ}, 1 \mathrm{~mm}\right.$ thick slices). 


\section{Analysis}

\section{Modifications to the orthogonal matching pursuit algorithm}

The OMP algorithm was slightly modified to better suit the current problem of MWF estimation from MR relaxation data. Since the relaxation signal and dictionary are strictly positive, the highest correlation of the signal with the dictionary is always found for the longest $\mathrm{T} 2$ relaxation time present in the dictionary (assuming all dictionary elements have unit amplitude). Therefore, the first iteration depends on the dictionary defined by the user, and does not necessarily converge to a meaningful answer. To improve the result of the OMP algorithm, two random elements are selected from the dictionary for the initialization of the iteration process. One initialization element corresponds to a myelin-water associated relaxation time (T2<40 ms) and the other element is one of the slower components (T2 $\geq 40$ $\mathrm{ms})$. Note that this initialization makes no assumption on the final number of components. The OMP tries to explain as much of the current residual as possible in each iteration, and therefore does not necessarily converge to the global optimum. Furthermore, due to the random initialization, the output of the algorithm can vary for identical inputs. Therefore, the stability of the algorithm can be enhanced by running with multiple random initializations per voxel. Since OMP is approximately 20 times faster than NNLS, we ran the OMP algorithm 20 times for each voxel. For the averaging of these results, an exponentially decaying quadratic weighting is applied, such that those MWFs with a larger fitting residual have a lower weighing, as they are likely the result of less optimal fitting due to the greedy nature of the OMP algorithm (Figure 3.1). Furthermore, the effect of the number of random initializations is investigated using the synthetic data by also running the OMP algorithm 1, 5,10 and 50 times.

The modified algorithm stops when the residual of the current iteration is larger than the residual of the previous iteration, in other words, when the next selected element does not provide a better fit. These aforementioned modifications were added to the Matlab implementation of the OMP algorithm by Yaghoobi [29], and it is available on Github (https://github.com/GSDrenthen/Non-Negative-OMP).

\section{Data Preprocessing}

A singular value decomposition filter was used to reduce noise in the measured multi-echo data [40]. Furthermore, the EPG algorithm was used to correct for B1-inhomogeneities [30]. To this end, for each voxel the corresponding refocusing flip angle is calculated by solving the multi-exponential problem for a range of flip angles, and subsequently selecting the flip angle that corresponds to the lowest residual. A refocusing flip angle range of $100-180^{\circ}$ was used. 
For the construction of the T2 basis functions using the EPG, the T1 relaxation was assumed to be $1000 \mathrm{~ms}$ in all cases.

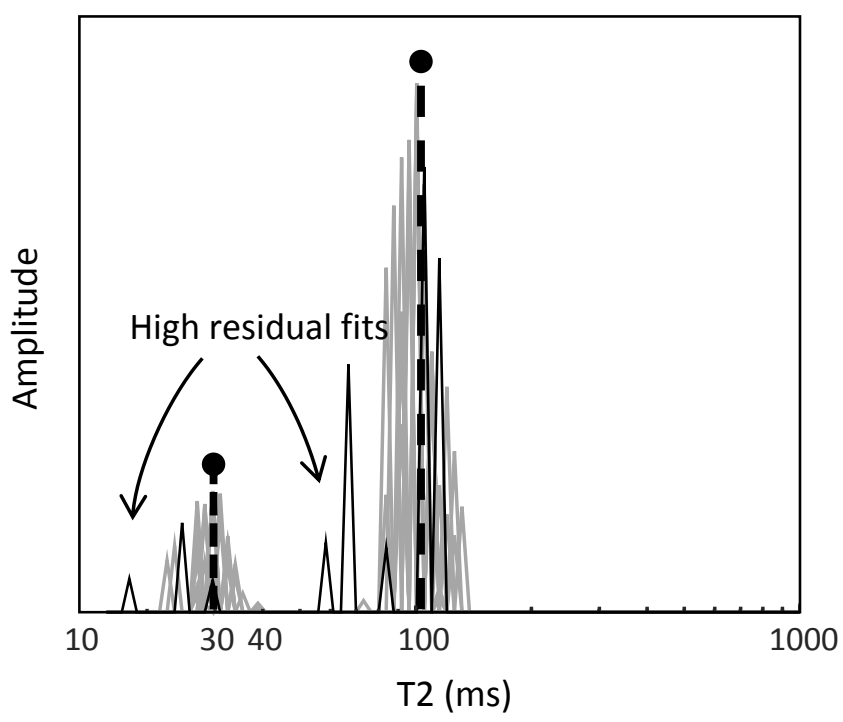

Figure 3.1: Example of a typical T2 distribution reconstructed using 20 iterations of the OMP. High residual fits (solid black lines) will contribute less to the final result in comparison to lower residual fits (gray lines). The two dashed black lines represent the original $\mathrm{T} 2$ distribution.

For the multi-exponential analysis, dictionaries of 120 and 1000 logarithmically spaced relaxation basis functions with a range of T2 relaxation times of $15-3500 \mathrm{~ms}$, was used for the NNLS and OMP methods, respectively. An upper bound of $3500 \mathrm{~ms}$ was chosen such that contribution from free water content (T2 $>2$ s) could also be fitted. Previously, it was shown that reliable fits could also be obtained using a 40 element basis set [32]. However, since we use a high resolution (1000 elements) basis set for the OMP algorithm we compare it to a relative high resolution (120 elements) NNLS. Furthermore, to investigate if the increased number of elements in the basis set has an effect on the MWF estimation, the OMP was also run with the same basis set of 120 functions as the NNLS. The curvature of the NNLS amplitude spectrum was minimized using a standard regularization, allowing a misfit of $1.020 \leq \chi_{\mathrm{reg}} / \chi_{\min } \leq 1.025$ [11]. Previously, regularization of the NNLS was associated with an underestimated MWF [41]. Therefore, to investigate the effect of the regularization strength, an additional numerical simulation with two extra smoothing constraints $(1.005 \leq$ $\chi_{\mathrm{reg}} / \chi_{\min } \leq 1.010$ and $\left.1.040 \leq \chi_{\mathrm{reg}} / \chi_{\min } \leq 1.045\right)$ was performed. 


\section{Validation}

For the numerical simulations and phantom model, the accuracy of the MWF estimation by the OMP and NNLS methods was evaluated using the absolute bias, defined as the mean absolute deviation from the ground truth MWF,

$$
\text { Absolute bias }=\frac{\sum_{1}^{N}\left|\mu-\mathrm{MWF}_{\text {in }}\right|}{N},
$$

where $\mu$ is the mean estimated MWF, MWF in is the ground truth MWF (range 0-30\%, with $1 \%$ increments) and $N$ is the number of MWF values ( $N=31$ in this case).

The precision of both methods is evaluated by the relative standard deviation (RSD),

$$
\operatorname{RSD}(\%)=100 \% \cdot \frac{\sigma}{\mu}
$$

where $\sigma$ is the standard deviation and $\mu$ is the calculated mean. The RSD is determined for a $\mathrm{MWF}_{\text {in }}$ of $15 \%$ (RSD $15 \%$ ), since a MWF of $15 \%$ is a commonly reported MWF in normal white matter [12], [14], [42].

As the in vivo data cannot be validated with a ground truth measurement, we compare the two methods with respect to each other. To this end, the white matter was segmented from the T1-weighted images and coregistered to the MSE space using the SPM12 software. Thereafter, two major white matter structures, the genu and splenium of the corpus callosum, were manually delineated, and two subcortical structures, the thalamus and the caudate, were segmented using the FreeSurfer software [43]. These regions of interest (ROIs) are important structures and have often been studied in previous MWF research [32], [44].

\section{Results}

\section{Numerical simulations}

The mean estimated MWF of the NNLS and OMP methods for the simulated data with an SNR of 200 at the first echo (TE $=12 \mathrm{~ms}$ ) is shown with respect to the supplied MWF in Figure 3.2. Additionally, the absolute bias of the MWF estimation is depicted in the same figure. Both methods systematically underestimate the ground truth MWF. However, over the whole MWF range, the MWF values estimated with the OMP are closer to the ground truth MWF compared to those estimated with the NNLS. This results in a 1.8 times smaller average absolute bias of the OMP method compared to the NNLS (NNLS: $4.4 \%$ vs. OMP: $2.5 \%$ ). The two methods have a comparable RSD $15 \%$ (NNLS: $29 \%$ vs. OMP: 32\%). 
Table 3.1: Absolute bias and $\mathrm{RSD}_{15 \%}$ of the MWF estimation of healthy white matter tissue and a white matter lesion using the NNLS and OMP algorithms with varying SNR.

\begin{tabular}{lccccccc}
\hline & & \multicolumn{3}{l}{ Healthy white matter } & \multicolumn{3}{c}{ White matter lesion } \\
\cline { 3 - 8 } & SNR & NNLS & OMP & NNLS/OMP & NNLS & OMP & NNLS/OMP \\
\hline Absolute bias & 100 & 6.4 & 3.9 & 1.6 & 2.9 & 2.1 & 1.4 \\
& 200 & 4.4 & 2.5 & 1.8 & 1.7 & 1.3 & 1.3 \\
& 350 & 3.0 & 1.6 & 1.9 & 1.0 & 0.7 & 1.4 \\
\hline RSD $15 \%$ & 100 & $57 \%$ & $49 \%$ & 1.2 & $34 \%$ & $31 \%$ & 1.1 \\
& 200 & $29 \%$ & $32 \%$ & 0.9 & $17 \%$ & $20 \%$ & 0.9 \\
& 350 & $19 \%$ & $22 \%$ & 0.9 & $10 \%$ & $14 \%$ & 0.7 \\
\hline
\end{tabular}

Abbreviations: MWF, myelin-water fraction; NNLS, non-negative least squares; OMP, orthogonal matching pursuit; SNR, signal-to-noise ratio; $\mathrm{RSD}_{15 \%}$, relative standard deviation for a $\mathrm{MWF}_{\text {in }}$ of $15 \%$.

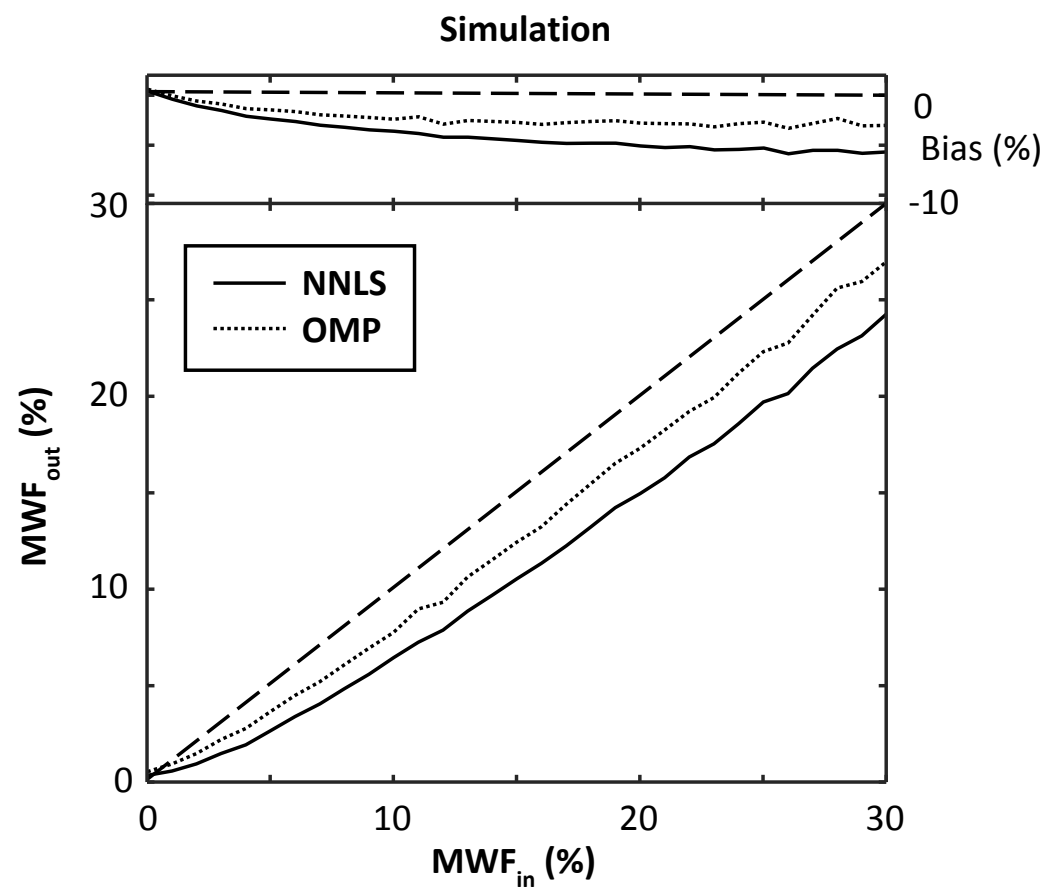

Figure 3.2: The estimated MWF from the numerical simulations using the NNLS (solid line) and the OMP (dotted line) is plotted against the supplied MWF. The dashed black line depicts the ground truth MWF, while the top of the figure shows the absolute bias of the estimated MWF.

The effect of SNR on the absolute bias and RSD $15 \%$ of the MWF estimation for both methods is shown in Table 3.1 for healthy white matter tissue and for white matter lesions. The absolute 
bias and RSD $15 \%$ increased for the simulations with lower SNR. For nearly all SNR levels, the OMP has a smaller absolute bias and similar RSD $15 \%$ compared to the NNLS.

The effect of echo spacing and T2myelin is shown in Table 3.2. A decreased T2myelin of 15 ms greatly improves the MWF estimation for both methods. The large improvement is most likely due to the two T2 peaks being further apart, and thus more easily distinguished. Reducing the echo spacing to $8 \mathrm{~ms}$ allows for a better characterization of the fast myelin decay, however also decreases the total T2 range (up to $256 \mathrm{~ms}$ ) possibly hampering the correct characterization of slower decaying signals.

Table 3.2: Absolute bias and $\mathrm{RSD}_{15 \%}$ of the MWF estimation for varying echo spacing and $\mathrm{T} 2_{\text {myelin }}$ peak using the NNLS and OMP algorithms with an SNR of 200.

\begin{tabular}{lcccc}
\hline & ESP/ T2myelin & NNLS & \multicolumn{1}{c}{ OMP } & NNLS/OMP \\
\hline Absolute bias & $8 / 30 \mathrm{~ms}$ & 5.9 & 3.0 & 2.0 \\
& $8 / 15 \mathrm{~ms}$ & 0.8 & 0.3 & 2.7 \\
& $12 / 15 \mathrm{~ms}$ & 1.1 & 0.5 & 2.0 \\
\hline RSD $15 \%$ & $8 / 30 \mathrm{~ms}$ & $27 \%$ & $33 \%$ & 0.8 \\
& $8 / 15 \mathrm{~ms}$ & $8 \%$ & $8 \%$ & 1.0 \\
& $12 / 15 \mathrm{~ms}$ & $12 \%$ & $11 \%$ & 1.1 \\
\hline
\end{tabular}

Abbreviations: MWF, myelin-water fraction; NNLS, non-negative least squares; OMP, orthogonal matching pursuit; $\mathrm{ESP}$, echo spacing; $\mathrm{RSD}_{15 \%}$, relative standard deviation for a $\mathrm{MWF}_{\text {in }}$ of $15 \%$.

Using a continuous T2 distribution instead of two distinct discrete peaks did not have a strong or meaningful effect on the results. The changes in absolute bias were $<0.1 \%$ for both methods, while the $\mathrm{RSD}_{15} \%$ did not deviate more than $1 \%$. Reducing the basis set of the OMP algorithm to 120 elements showed similar changes in absolute bias $(<0.1 \%)$ and $\mathrm{RSD}_{15 \%}(<1 \%)$. Furthermore, in Figure 3.3 the effect of computational time on the estimation of the MWF is shown for the OMP method, where the absolute bias decreases with running more instances of the algorithm.

Lastly, the effect of the NNLS smoothing constraint is shown in Figure 3.4. It is observed that a stronger regularization (i.e. higher smoothing constraint) shifts the peaks of the T2 distribution slightly to the lower T2 values. Additionally, the estimated MWF is lower for stronger smoothing (e.g. the total myelin-related amplitude decreases). 


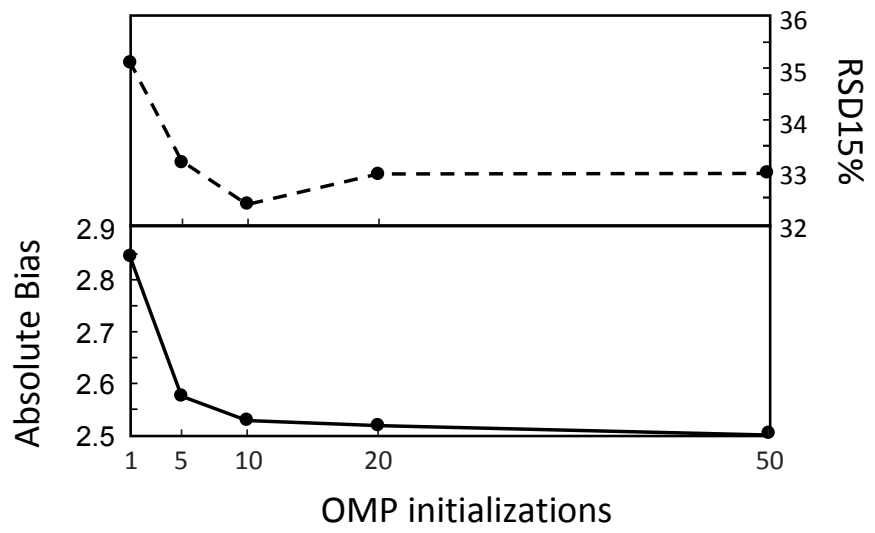

Figure 3.3: The effect that the number of random initializations has on the MWF estimation of the OMP method. Dashed line represents $\mathrm{RSD}_{15 \%}$ and the solid line depicts the absolute bias.

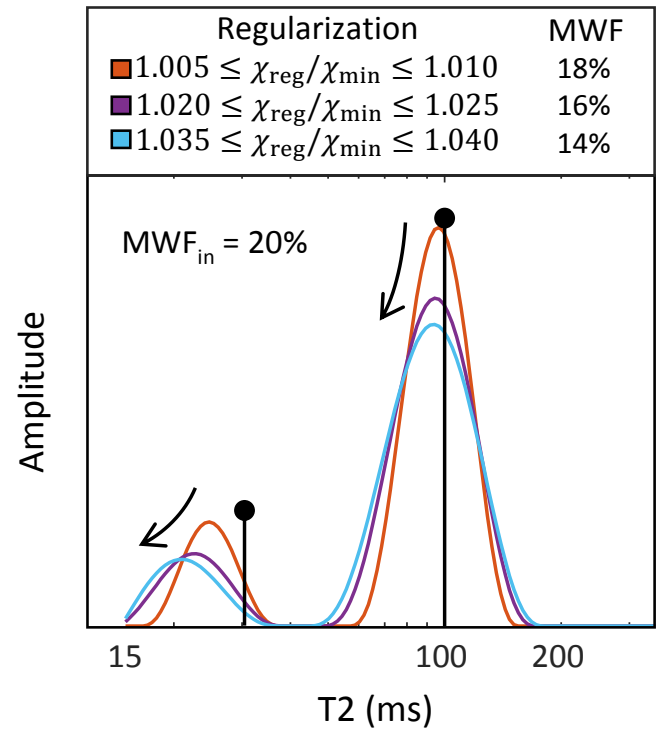

Figure 3.4: The effect of non-negative least squares regularization using a smoothing constraint. The colored lines depict the reconstructed $\mathrm{T} 2$ distribution for different regularization strengths, while the black lines represent the original $\mathrm{T} 2$ distribution.

\section{Phantom model}

The SNR of the phantom measurement at the first echo time (TE $=12 \mathrm{~ms}$ ) was $340 \pm 34$ (mean $\pm \mathrm{SD}$ ). The mean estimated MWF of the NNLS and OMP methods for the phantom measurements is shown in Figure 3.5 with respect to the defined MWF. Additionally, the absolute bias of the MWF estimation is shown for both methods in the same figure. From this, 
we observe that over almost the whole MWF range both methods systematically underestimate the ground truth MWF, and that the MWF values estimated with the OMP are closer to the ground truth MWF. The absolute bias of the OMP method is on average 1.7 times smaller compared to the NNLS (NNLS: $3.4 \%$ vs. OMP: $2.0 \%$ ), while the two methods have a comparable RSD $15 \%$ (NNLS: 25\% vs. OMP: 18\%).

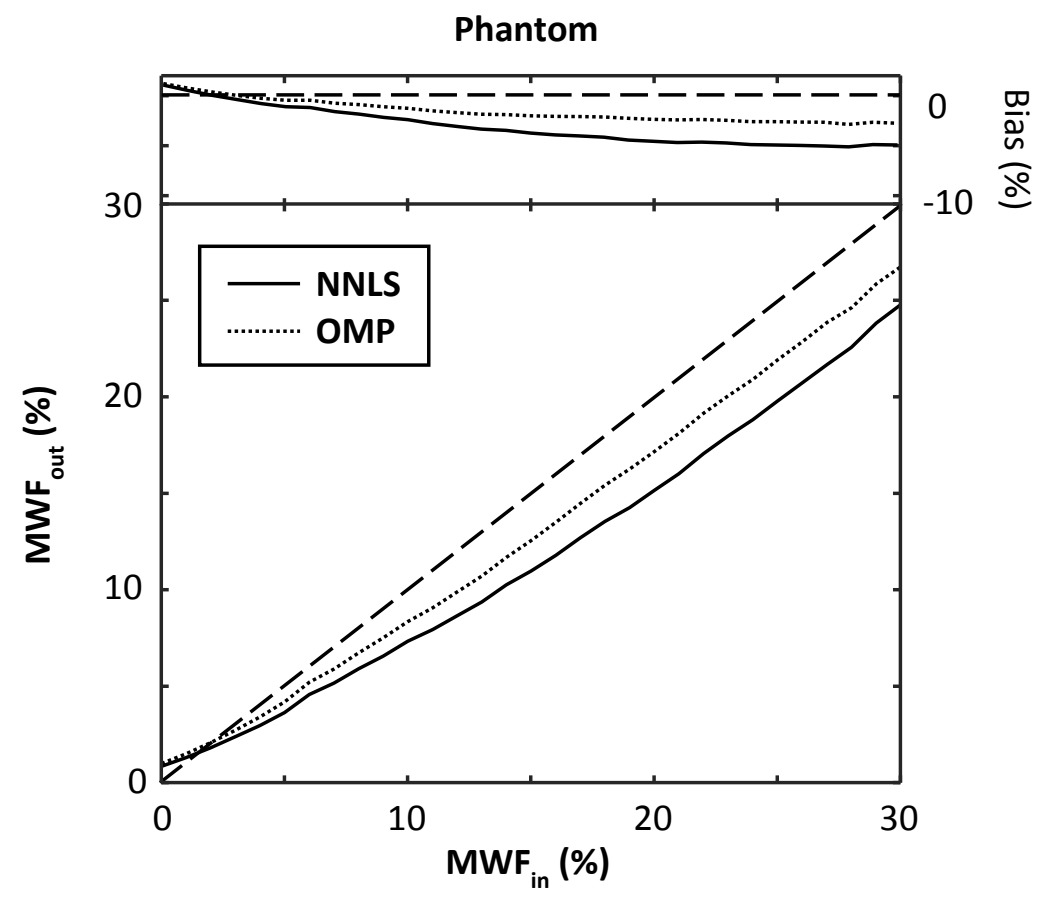

Figure 3.5: The estimated MWF from the phantom model using the NNLS (solid line) and the OMP (dotted line) is plotted against the defined MWF. The dashed black line depicts the ground truth MWF, while the top of the figure shows the absolute bias of the estimated MWF.

\section{In vivo}

The SNR of the in vivo measurements at the first echo time (TE $=12 \mathrm{~ms}$ ) was $363 \pm 193$ (mean $\pm \mathrm{SD}$ ) measured in the genu and splenium of the corpus callosum. In Figure 3.6A the MWF maps of a single representative healthy subject calculated with both the NNLS and OMP method are shown. In Figure 3.6B the T1-weighted image with the ROIs is shown, and in Figure 3.6C a scatter plot of voxel-wise MWF values in the ROIs as well as all the white matter voxels is shown. Mean MWF values obtained in the ROIs and white matter with the OMP algorithm were, on average, slightly higher by approximately $2 \%$ (actual MWF units) in comparison to the NNLS (Table 3.3). In addition, previously reported MWF values in the genu, splenium, thalamus and caudate using the MSE acquisition and NNLS with EPG correction as in the current study are also given in the table. 
Using the 3D GRASE sequence, whole brain MWF maps are calculated with the NNLS and OMP. Figure 3.7A shows the MWF map estimated using the OMP algorithm. In Figure 3.7B, a scatter plot of voxel-wise MWF values in the ROIs as well as all the white matter voxels is shown. On average, the MWF values obtained with the OMP algorithm were slightly higher by approximately 1 to $2 \%$ (actual MWF units) in comparison to NNLS.

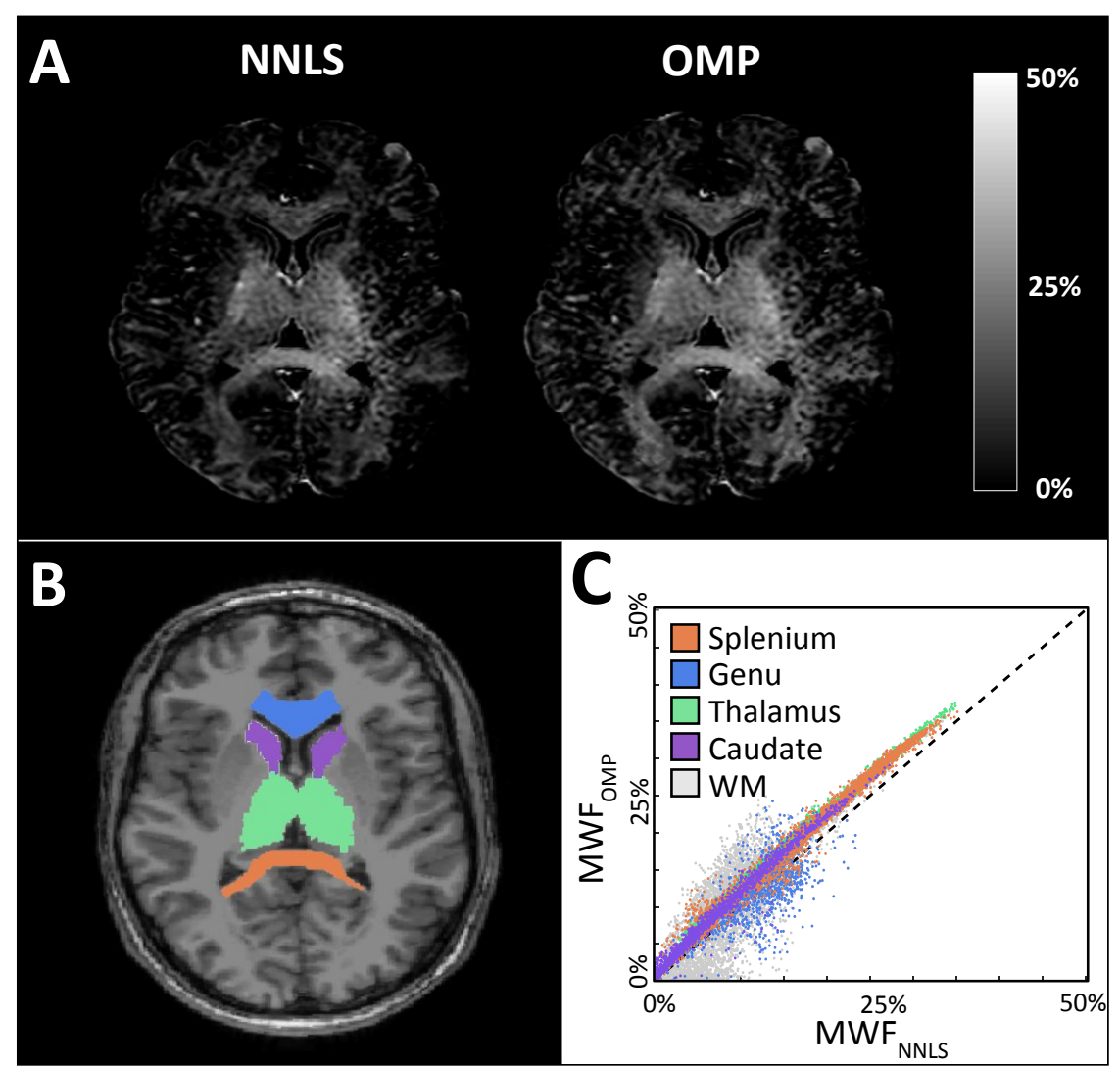

Figure 3.6: The results of the MSE sequence of a single representative subject (male, 28 year). The MWF map estimated with the NNLS and OMP methods (A), the T1-weighted image with the delineated ROIs (B) and a scatter plot of the MWF values in the ROIs and all WM (C) are shown. 
Table 3.3: The MWF estimated with the NNLS and OMP algorithms in the two ROIs (genu and splenium of the corpus callosum) as well as all WM. For reference, previously reported MWF values are added. Means and standard deviations are given.

\begin{tabular}{llllll}
\hline \multirow{5}{*}{ Average MWF (\%) } & \multicolumn{3}{l}{ Literature values } & \\
\cline { 2 - 6 } & NNLS & OMP & Prasloski [32] & Prasloski [12] & Mädler [44] \\
\hline Splenium & $18.8 \pm 5.6$ & $21.1 \pm 5.6$ & $15.2 \pm 2.2^{\mathrm{a}}$ & $14.5 \pm 2.0^{\mathrm{a}}$ & $10.8 \pm 5.6$ \\
Genu & $11.4 \pm 5.2$ & $13.2 \pm 5.3$ & $10.5 \pm 2.4^{\mathrm{a}}$ & $10.6 \pm 3.6^{\mathrm{a}}$ & $9.0 \pm 4.0$ \\
Thalamus & $14.3 \pm 3.8$ & $17.0 \pm 3.9$ & - & $3.4 \pm 2.1^{\mathrm{a}}$ & $3.1 \pm 0.7$ \\
Caudate & $5.6 \pm 1.7$ & $7.1 \pm 1.8$ & - & $1.8 \pm 1.2^{\mathrm{a}}$ & $2.4 \pm 0.1$ \\
All WM & $11.3 \pm 6.2$ & $13.0 \pm 6.7$ & - & - & $9.9 \pm 3.6$ \\
\hline
\end{tabular}

Abbreviations: MWF, myelin-water fraction; NNLS; non-negative least squares; OMP, orthogonal

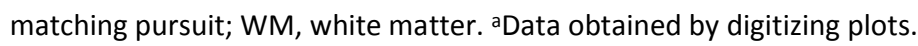

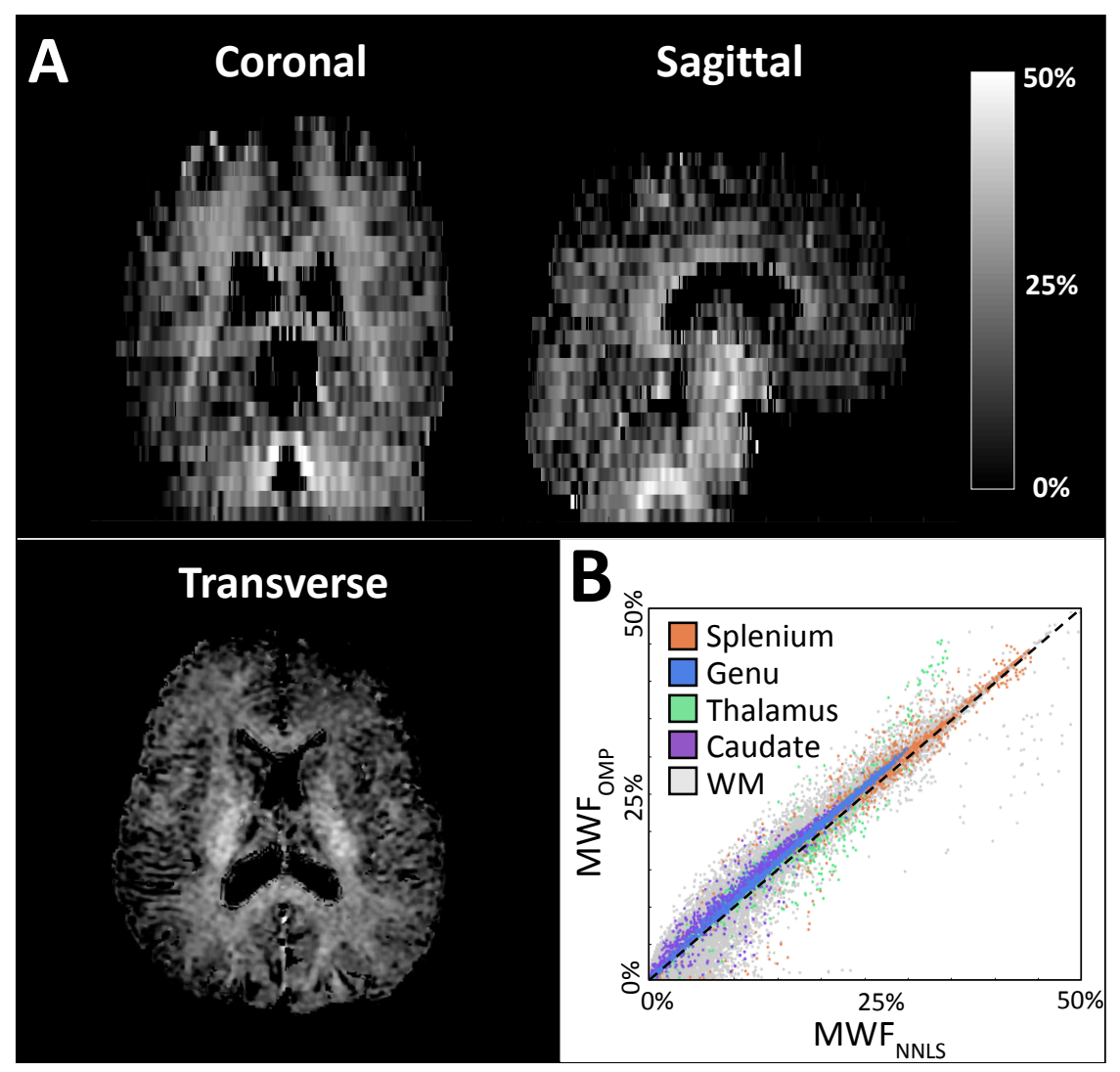

Figure 3.7: The results of the 3D GRASE sequence of a single subject (female, 29 year). A coronal, sagittal and transverse slice of the MWF map estimated with the OMP method (A) and a scatter plot of the MWF values in the ROIs and all WM (B) are shown. 


\section{Discussion}

\section{Current findings}

In the current study, the OMP algorithm is introduced as an alternative method for the determination of the MWF from multi-exponential T2 relaxation data, and it is compared with the most commonly applied method in literature, NNLS. The estimation of the MWF using both methods is validated using a ground truth from numerical simulations and an in vitro phantom model. It is shown that the OMP yields a more accurate MWF estimation compared to the NNLS, while a comparable precision (in terms of RSD ${ }_{15 \%}$ ) is obtained. Furthermore, the in vivo results are in line with the numerical simulations and the phantom model, showing, on average, that the MWF calculated with the OMP is higher compared to the NNLS.

\section{Effect of SNR}

For each of the three SNR levels studied, the OMP outperforms the NNLS algorithm in terms of a smaller absolute bias. As to be expected, a lower SNR is found to be associated with a larger overall absolute bias as well as a less precise measurement (i.e. higher RSD $15 \%$ ) of the MWF. The multi-echo sequence used in this study has an approximate SNR of 350. Therefore, we would expect that the simulations with an SNR of 350 would best represent the phantom and in vivo results. Indeed, the phantom model shows an accuracy and precision comparable to the simulations with SNR 350. The absolute bias is, however, higher in the phantom model. Most likely this is caused by the B1 inhomogeneities and subsequent error correction using the EPG algorithm, which induces additional errors which are difficult to model in the simulations.

\section{Underestimation of MWF}

The MWF is systematically underestimated by an absolute bias of approximately $1-5 \%$ in the simulations as well as the phantom model. One possible explanation for this phenomenon might be the effect of Rician noise present in magnitude images. While the Rician noise can be considered near-Gaussian for higher SNRs, it tends towards the Rayleigh distribution for images with low SNR. As the SNR in multi-echo measurements decreases exponentially for longer echo times, the Rician noise in the low SNR measurements could be misinterpreted for a slow decaying component. This artificial slow decaying component adds to the total water amplitude and therefore reduces the MWF [15].

Previously, the regularization of the NNLS algorithm was also found to cause MWF underestimation [41]. In this study, it is observed that the peak of the underlying T2 distribution shifts to a shorter T2. Since the regularization introduces a smoothing constraint, 
more elements from the basis set will be used to obtain an optimal result. To provide a result with the lowest misfit, elements that are close to the original peak will be preferred. Since the basis set is logarithmically spaced, the shorter T2 times are closer to the original peak, shifting the peak to the left in the spectrum. To compensate for this shift, the amplitude of the peak is reduced. This effect is stronger for the myelin-peak, as in this region the basis functions are more densely distributed, thus underestimating the MWF. Using NNLS with weaker regularization, or even unregularized, would suffer less from this underestimation [41], however it would decrease the stability of the algorithm, as it is well established that the illposed NNLS requires strong regularization.

The OMP is not regularized with a smoothing constraint, instead, the OMP algorithm is repeated 20 times to provide more stable results. Therefore, the OMP does not suffer from an underestimated MWF due to a smoothing constraint. Since the OMP has a (roughly 20 times) lower computational complexity, it results in a computational time comparable to the NNLS with 120 basis functions. However, the NNLS is commonly used with fewer basis functions, resulting in a lower computational time (e.g. the 40 basis functions case is roughly 1.5 times faster). Therefore, the comparison of computational times presented here should be interpreted with caution.

\section{In vivo applicability}

The in vivo results are in line with the numerical simulations and phantom measurements, as we found that the in vivo MWF estimated with the OMP yielded somewhat higher values compared to the NNLS. More specifically, since the in vivo data have an SNR of approximately 350 we would expect a similar increase of the OMP as measured in the simulations with SNR 350. Indeed, we found that the mean MWF values determined with the OMP in the genu and splenium of the corpus callosum, thalamus, caudate and all the white matter show a similar increase of MWF compared to those estimated with the NNLS. Furthermore, the MWF values reported in the genu and splenium of the corpus callosum using the NNLS method are within normal limits compared to previous studies [32], [44], whereas the MWF values reported in the thalamus and caudate are much higher. However, while the values are not specifically reported, some other studies have also observed a relative high MWF in these structures [41], [45]. Therefore, these discrepancies between the MWF values reported in our study and the values of previous literature might be explained by differences in age of the healthy volunteers and different scanning parameters.

\section{Study considerations}

In this study we used healthy volunteers without white matter abnormalities. However, our simulations have indicated that the OMP algorithm outperforms the NNLS in healthy tissue as well as in white matter lesions. This implies that the OMP algorithm can be a useful tool in 
future patient studies. The single-slice MSE sequence that was used in this study limits the comparison of the MWF estimation methods to specific brain regions. Furthermore, the MSE sequence is a relatively slow sequence ( $13 \mathrm{~min}$ for a single slice) and therefore not optimal for patient studies. However, the MSE is considered as the reference method for MWF estimation [11], and is therefore suitable for comparative studies as the current study. The performance of the OMP algorithm to determine the MWF should be further evaluated on accelerated multi-echo sequences. Recently, several studies have already focused on fast acquisition of multi-echo data for the purpose of MWF mapping. For instance, Prasloski et al. showed that whole brain MWF mapping was possible under 15 minutes using a gradient-spin echo sequence with 32 echo times [12], while Nguyen et al. showed that even faster whole brain MWF mapping was possible acquiring 6 echo times in 4 minutes using a modified T2prep sequence [46]. In this study we already showed the applicability of the OMP algorithm for multi-echo data acquired using a 3D GRASE sequences. Furthermore, the OMP algorithm could also prove valuable in other MR modalities that require multi-exponential analysis, such as intravoxel incoherent motion [47], [48].

\section{Conclusions}

We have applied the OMP for the multi-exponential T2 relaxation component analysis to estimate the MWF and compared it to the most commonly applied algorithm in literature, the NNLS. Using numerical simulations and an in vitro phantom model it was demonstrated that the OMP is a more accurate method for the estimated MWF. The bias of the MWF estimation using the OMP was roughly reduced by a factor of two, while the precision was comparable to the NNLS. In vivo results show similar findings as the simulations and phantom model, proving that OMP can be a preferred alternative to conventional methods for its accuracy and low computational complexity. 


\section{References}

[1] J. L. Holtrop, T. M. Loucks, J. J. Sosnoff, and B. P. Sutton, “Investigating Age-related changes in fine motor control across different effectors and the impact of white matter integrity," Neuroimage, vol. 96, pp. 81-87, Aug. 2014.

[2] M. Simons and K. Nave, "Oligodendrocytes: Myelination and Axonal Support," Cold Spring Harb. Perspect. Biol., vol. 8, p. a020479, Jan. 2016.

[3] K. Nave and H. B. Werner, "Myelination of the Nervous System: Mechanisms and Functions," Annu. Rev. Cell Dev. Biol., vol. 30, pp. 503-533, Oct. 2014.

[4] M. F. Glasser and D. C. Van Essen, "Mapping Human Cortical Areas In Vivo Based on Myelin Content as Revealed by T1- and T2-Weighted MRI," J. Neurosci., vol. 31, no. 32, pp. 11597-11616, Aug. 2011.

[5] C. Laule et al., "Magnetic resonance imaging of myelin," Neurotherapeutics, vol. 4, no. 3, pp. 460484, Jul. 2007.

[6] M. A. van Buchem et al., "Global estimation of myelination in the developing brain on the basis of magnetization transfer imaging: a preliminary study," Am. J. Neuroradiol., vol. 22, no. 4, pp. 762-6, Apr. 2001.

[7] I. M. Vavasour, C. Laule, D. K. B. Li, A. L. Traboulsee, and A. L. Mackay, "Is the Magnetization Transfer Ratio a Marker for Myelin in Multiple Sclerosis?," J. Magn. Reson. Imaging, vol. 33, pp. 713718, 2011.

[8] V. Sheth et al., "Magnetic resonance imaging of myelin using ultrashort Echo time (UTE) pulse sequences: Phantom, specimen, volunteer and multiple sclerosis patient studies," Neuroimage, vol. 136, pp. 37-44, Aug. 2016.

[9] A. C. Seifert, C. Li, M. J. Wilhelm, S. L. Wehrli, and F. W. Wehrli, "Towards quantification of myelin by solid-state MRI of the lipid matrix protons," Neuroimage, vol. 163, pp. 358-367, Dec. 2017.

[10] S. C. L. Deoni, B. K. Rutt, T. Arun, C. Pierpaoli, and D. K. Jones, "Gleaning multicomponent T1 and T2 information from steady-state imaging data," Magn. Reson. Med., vol. 60, no. 6, pp. 1372-1387, Dec. 2008.

[11] E. Alonso-Ortiz, I. R. Levesque, and G. B. Pike, "MRI-based myelin water imaging: A technical review," Magn. Reson. Med., vol. 73, no. 1, pp. 70-81, Jan. 2015.

[12] T. Prasloski et al., "Rapid whole cerebrum myelin water imaging using a 3D GRASE sequence," Neuroimage, vol. 63, pp. 533-539, Oct. 2012.

[13] C. Laule, P. Kozlowski, E. Leung, D. K. B. Li, A. L. Mackay, and G. R. W. Moore, “Myelin water imaging of multiple sclerosis at 7T: Correlations with histopathology," Neuroimage, vol. 40, pp. 1575-1580, 2008.

[14] S. H. Kolind, B. Mädler, S. Fischer, D. K. B. Li, and A. L. MacKay, "Myelin water imaging: Implementation and development at 3.0T and comparison to 1.5T measurements," Magn. Reson. Med., vol. 62, no. 1, pp. 106-115, 2009.

[15] T. A. Bjarnason, C. R. Mccreary, J. F. Dunn, and J. R. Mitchell, "Quantitative T 2 Analysis: The Effects of Noise, Regularization, and Multivoxel Approaches," Magn. Reson. Med., vol. 63, no. October 2009, pp. 212-217, 2010.

[16] K. P. Whittall and A. L. MacKay, "Quantitative interpretation of NMR relaxation data," J. Magn. Reson., vol. 84, no. 1, pp. 134-152, Aug. 1989.

[17] G. Davis, S. Mallat, and Z. Zhang, "Adaptive time-frequency decompositions," Opt. Eng., vol. 33, no. 7, pp. 2183-2191, 1994. 
[18] H. S. Goklani, J. N. Sarvaiya, and A. M. Fahad, "Image reconstruction using Orthogonal Matching Pursuit (OMP) algorithm," in 2nd International Conference on Emerging Technology Trends in Electronics, Communication and Networking, 2014.

[19] P. Bouboulis, G. Papageorgiou, and S. Theodoridis, "Robust image denoising in RKHS via orthogonal matching pursuit," in 4th International Workshop on Cognitive Information Processing (CIP), 2014.

[20] Y. C. Pati, R. Rezaiifar, and P. S. Krishnaprasad, "Orthogonal matching pursuit: recursive function approximation with applications to wavelet decomposition," in Proceedings of 27th Asilomar Conference on Signals, Systems and Computers, 1993, pp. 40-44.

[21] K. Kazimierczuk and P. Kasprzak, "Modified OMP Algorithm for Exponentially Decaying Signals," Sensors, vol. 15, pp. 234-247, Dec. 2014.

[22] E. Esser, Y. Lou, and J. Xin, "A Method for Finding Structured Sparse Solutions to Nonnegative Least Squares Problems with Applications," SIAM J. Imaging Sci., vol. 6, no. 4, pp. 2010-2046, Jan. 2013.

[23] C. L. Lawson and R. J. Hanson, Solving Least Squares Problems. Society for Industrial and Applied Mathematics, 1995.

[24] E. P. Minty, T. A. Bjarnason, C. Laule, and A. L. Mackay, “Myelin Water Measurement in the Spinal Cord," Magn. Reson. Med., vol. 61, no. 2, pp. 883-892, 2009.

[25] C. Lenz, M. Klarhöfer, and K. Scheffler, "Feasibility of in vivo myelin water imaging using 3D multigradient-echo pulse sequences," Magn. Reson. Med., vol. 68, pp. 523-528, 2012.

[26] E. L. Macmillan et al., "Myelin water and T2 relaxation measurements in the healthy cervical spinal cord at 3.0T: Repeatability and changes with age," Neuroimage, vol. 54, pp. 1083-1090, 2011.

[27] C. Laule, S. H. Kolind, T. A. Bjarnason, D. K. B. Li, and A. L. MacKay, "In vivo multiecho T2 relaxation measurements using variable TR to decrease scan time," Magn. Reson. Imaging, vol. 25, no. 6, pp. 834-839, 2007.

[28] A. M. Bruckstein, M. Elad, and M. Zibulevsky, "On the Uniqueness of Nonnegative Sparse Solutions to Underdetermined Systems of Equations," IEEE Trans. Inf. Theory, vol. 54, no. 11, pp. 4813-4820, Nov. 2008.

[29] M. Yaghoobi, D. Wu, and M. E. Davies, “Fast Non-Negative Orthogonal Matching Pursuit,” IEEE Signal Process. Lett., vol. 22, no. 9, pp. 1229-1233, Sep. 2015.

[30] J. Hennig, "Echoes -How to Generate, Recognize, Use or Avoid Them in MR-Imaging Sequences Part I : Fundamental and Not So Fundamental Properties of Spin Echoes," Concepts Magn. Reson., vol. 3, pp. 125-143, 1991.

[31] A. Akhondi-Asl, O. Afacan, M. Balasubramanian, R. V. Mulkern, and S. K. Warfield, "Fast myelin water fraction estimation using 2D multislice CPMG," Magn. Reson. Med., vol. 76, no. 4, pp. 13011313, Oct. 2016.

[32] T. Prasloski, B. Mädler, Q. S. Xiang, A. MacKay, and C. Jones, "Applications of stimulated echo correction to multicomponent T2 analysis," Magn. Reson. Med., vol. 67, no. 6, pp. 1803-1814, 2012.

[33] C. Laule et al., "Long T2 water in multiple sclerosis: What else can we learn from multi-echo T2 relaxation?," J. Neurol., vol. 254, no. 11, pp. 1579-1587, Nov. 2007.

[34] P. Brex et al., "Lesion heterogeneity in multiple sclerosis: a study of the relations between appearances on T1 weighted images, T1 relaxation times, and metabolite concentrations," J Neurol Neurosurg Psychiatry, vol. 68, pp. 627-632, 2000.

[35] A. MacKay, C. Laule, I. Vavasour, T. Bjarnason, S. Kolind, and B. Mädler, "Insights into brain microstructure from the T2 distribution," Magn. Reson. Imaging, vol. 24, no. 4, pp. 515-525, 2006. 
[36] S. Webb, C. A. Munro, R. Midha, and G. J. Stanisz, "Is Multicomponent T 2 a Good Measure of Myelin Content in Peripheral Nerve?," Magn. Reson. Med., vol. 49, pp. 638-645, 2003.

[37] R. M. Lebel and A. H. Wilman, "Transverse relaxometry with stimulated echo compensation," Magn. Reson. Med., vol. 64, no. 4, pp. 1005-1014, Jun. 2010.

[38] S. Aja-fernández, C. Alberola-lópez, and C. Westin, "Noise and Signal Estimation in Magnitude MRI and Rician Distributed Images: A LMMSE Approach," IEEE Trans. image Process., vol. 17, no. 8, pp. 1383-1398, 2008.

[39] A. Mackay, K. Whittall, J. Adler, D. Li, D. Paty, and D. Graeb, “In vivo visualization of myelin water in brain by magnetic resonance," Magn. Reson. Med., vol. 31, no. 6, pp. 673-677, Jun. 1994.

[40] M. Bydder and J. Du, "Noise reduction in multiple-echo data sets using singular value decomposition," Magn. Reson. Imaging, vol. 24, no. 7, pp. 849-856, Sep. 2006.

[41] J. Guo, Q. Ji, and W. E. Reddick, "Multi-slice myelin water imaging for practical clinical applications at 3.0 T," Magn. Reson. Med., vol. 70, no. 3, pp. 813-822, Sep. 2013.

[42] D. Kumar, S. Siemonsen, C. Heesen, J. Fiehler, and J. Sedlacik, “Noise robust spatially regularized myelin water fraction mapping with the intrinsic B1-error correction based on the linearized version of the extended phase graph model," J. Magn. Reson. Imaging, vol. 43, no. 4, pp. 800-817, Apr. 2016.

[43] B. Fischl, "Automatically Parcellating the Human Cerebral Cortex," Cereb. Cortex, vol. 14, no. 1, pp. 11-22, Jan. 2004.

[44] B. Mädler, S. A. Drabycz, S. H. Kolind, K. P. Whittall, and A. L. Mackay, "Is diffusion anisotropy an accurate monitor of myelination?. Correlation of multicomponent T2 relaxation and diffusion tensor anisotropy in human brain," Magn. Reson. Imaging, vol. 26, no. 7, pp. 874-888, 2008.

[45] D. Hwang, D. Kim, and Y. P. Du, "In vivo multi-slice mapping of myelin water content using T2* decay," Neuroimage, vol. 52, pp. 198-204, 2010.

[46] T. D. Nguyen et al., "Feasibility and reproducibility of whole brain myelin water mapping in 4 minutes using fast acquisition with spiral trajectory and adiabatic T2prep (FAST-T2) at 3T," Magn. Reson. Med., vol. 76, no. 2, pp. 456-465, Aug. 2016.

[47] D. Le Bihan, E. Breton, D. Lallemand, P. Grenier, E. Cabanis, and M. Laval-Jeantet, "MR imaging of intravoxel incoherent motions: application to diffusion and perfusion in neurologic disorders.," Radiology, vol. 161, no. 2, pp. 401-407, Nov. 1986.

[48] V. C. Keil et al., "Intravoxel Incoherent Motion MRI in the Brain: Impact of the Fitting Model on Perfusion Fraction and Lesion," J. Magn. Reson. Imaging, vol. 46, pp. 1187-1199, 2017. 



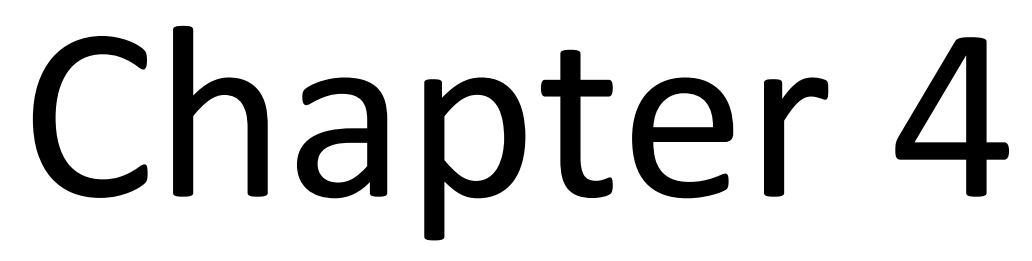

\section{Applicability and reproducibility of 2D multi-slice GRASE myelin-water fraction with varying acquisition acceleration}

Gerhard S. Drenthen, Walter H. Backes, Albert P. Aldenkamp, Jacobus F.A. Jansen.

NeuroImage 2019; 195:333-339. DOI: 10.1016/j.neuroimage.2019.04.011 


\section{Abstract}

Non-invasive quantification of the in vivo myelin content may provide valuable information regarding healthy maturation of the brain, as well as insights into demyelination of several neurological disorders. However, these scans are often long thereby limiting acquisition of large brain parts in clinically feasible acquisition times. Therefore, fast acquisition of whole brain myelin content is important. To avoid errors related to slice-selective pulses, most of the previous whole brain studies on myelin content relied on a 3D acquisition. However, multi-slice (2D) acquisition methods are often faster, and less susceptible to motion artifacts. Therefore, multi-slice approaches can be beneficial in a clinical setting.

We investigated the applicability and reproducibility of whole brain multi-slice GRASE myelin-water imaging with post-acquisition slice-profile correction in healthy volunteers (aged 25-32y). The applicability was evaluated using the agreement between the multi-slice GRASE and the reference method for myelin-water imaging, single-slice multi spin-echo (MSE) acquisition. Additionally, we assessed the effect of varying acquisition acceleration using parallel imaging on the reproducibility values.

First, the multi-slice myelin-water maps showed good agreement with the single-slice reference method, with a bias of at most $1.2 \%$ in absolute MWF values. Second, we found an average within-subject coefficient of variation $(\mathrm{CoV})$ of $5.9 \%$ and an average intra-class correlation coefficient (ICC) of .90 for myelin-water estimation using a multi-slice GRASE sequence without parallel acceleration (scan time 14:06 $\mathrm{min}$ ), while acquisition with a parallel acceleration factor of 2 resulted in a slightly worse average within-subject $\mathrm{CoV}$ of $6.4 \%$ and an average ICC of .83 at half the scan time. A multi-slice GRASE acquisition with parallel acceleration factor 2 and a scan time of 7:30 min still provides an excellent reproducibility of myelin-water values. 


\section{Introduction}

Myelin is a layered, fatty substance wrapped around the axons that is comprised of lipids and proteins. The myelin acts as an electrical insulator, accelerating the transport of electrical signals along the axons. Myelination is a vital process for a healthy neuronal maturation and development. Disruptions in the myelin content can have severe consequences and are related to several neurological disorders [1]. Non-invasive quantification of the in vivo myelin content may provide valuable information regarding healthy maturation of the brain, as well as insights into demyelination of several neurological disorders.

Magnetic resonance imaging (MRI) is a technique which is sensitive to cerebral white matter abnormalities and has therefore extensively been used to investigate the myelin content. For example, diffusion weighted imaging (DWI), T1/T2 ratio imaging, and magnetization transfer imaging have previously been used to assess the myelin content indirectly [2]-[4]. While these methods provide markers that are related to the myelin content, they are not specific to the myelin content [5], [6]. Direct quantification of the myelin sheath can be achieved by ultra-short echo time (UTE) imaging [7], however these sequences remain challenging on clinical MR systems due to hardware limitations [8]. Alternatively, myelin-water imaging using T2 relaxometry [9] or steady-state methods (mcDESPOT) [10] have been applied to quantify the water components between the bilayers of the myelin sheath. These methods provide a specific marker of myelin content as water is an important component making up $40 \%$ of the total myelin volume [11].

Using T2 relaxometry techniques, the relaxation of the MRI signal due to dephasing of water protons can be used to determine the myelin-water content. A number of different water compartments are present in brain tissue, each with a distinct T2 relaxation time. Water trapped between the bilayers of the myelin obeys a faster T2 relaxation $(15<\mathrm{T} 2<40$ $\mathrm{ms})$ compared to more freely moving water in the intra- and extracellular spaces $(80<\mathrm{T} 2<$ $100 \mathrm{~ms}$ ) and cerebrospinal fluid (CSF) (T2 > $1000 \mathrm{~ms}$ ). Multi-echo T2 signal decay measurements are composed of a superposition of the signals from all water compartments. For myelin-water imaging it is relevant to determine the shortest T2, myelin-water, component [11]. Subsequently, myelin content can be quantified by taking the fraction of myelin-water signal to the total signal, the so-called myelin-water fraction (MWF).

For MWF imaging to become more clinically feasible in a variety of populations and disorders, a rapid whole brain coverage with reproducible results is essential. Often, rapid whole brain coverage is achieved by accelerating image acquisition using parallel imaging techniques. However, these techniques introduce noise which can hamper the quality and consequently the reproducibility of the results. Currently, the impact of parallel imaging on the reproducibility is underdetermined. Moreover, whole brain MWF quantification has mainly focused on 3D sequences which do not suffer from imperfect slice profiles caused by slice-selective excitation pulses [12]. However, 3D imaging techniques do, in general, require 
longer acquisition times compared to multi-slice (2D) techniques and are more susceptible to motion artifacts. Therefore, multi-slice imaging with a correction for imperfect slice profiles has previously been introduced as a method for fast MWF quantification [13]-[17]. Guo et al. and Kumar et al. avoided the imperfect slice profile in multi-slice imaging by using a refocusing slice thickness three times the size of the excitation slice. However, this limits the number of slices (about 5) that can be acquired at once. Instead, previous studies used a post-acquisition correction by estimating the imperfect slice profile [15]-[17].

In this study, we evaluate the use of multi-slice gradient- and spin-echo (GRASE) sequences with slice profile correction to quantitatively map the in vivo MWF. First, the applicability is evaluated using the agreement of the multi-slice GRASE and the reference method for myelin-water imaging, a single-slice multi spin-echo (MSE) acquisition. Subsequently, the reproducibility of the multi-slice GRASE MWF estimation, as well as the effect of accelerated image acquisition through parallel imaging is investigated.

\section{Materials and Methods}

\section{MRI data acquisition}

Six volunteers (mean age 28y, range 25-32y, 3 males) were scanned on a 3 Tesla unit (Philips Achieva, Best, the Netherland) using a 32-element head coil. Informed consent was obtained from all volunteers prior to inclusion. First, for anatomical reference, T1-weighted 3D turbo field echo images were acquired (repetition time $(\mathrm{TR})=8.14 \mathrm{~ms}$, echo time $(\mathrm{TE})=3.73 \mathrm{~ms}$, inversion time $=1010 \mathrm{~ms}$, flip angle $=8^{\circ}$, voxel size $1 \mathrm{~mm}^{3}$ ). As a reference, single transverse slice MSE images were acquired $(\mathrm{TR}=3000 \mathrm{~ms}, 32$ echoes with the shortest possible echo spacing for this sequence of $12 \mathrm{~ms}$, range 12-384 ms, field of view $240 \times 198 \times 4 \mathrm{~mm}$, matrix $160 \times 132$, voxel size $1.5 \times 1.5 \times 4 \mathrm{~mm}$ and 2 signal averages) for comparison to the multi-slice MWF images [11], [18]. For segmentation of major white matter fiber bundles, DWI was performed $\left(\mathrm{TR}=7012 \mathrm{~ms}\right.$, TE $=74 \mathrm{~ms}$, voxel size $2 \mathrm{~mm}^{3}$, b value $1200 \mathrm{~s} / \mathrm{mm}^{2}, 66$ gradient directions and a single non-diffusion weighted $b=0$ image, acquisition time 8:03 min).

To determine the MWF reproducibility of multi-slice GRASE imaging, for every volunteer two GRASE images were acquired $(\mathrm{TR}=3000 \mathrm{~ms}, 32$ echoes with $10 \mathrm{~ms}$ echo spacing, range 10-320 ms, EPI factor $=3$, Turbo factor $=32$, 26 slices, field of view $240 \times 198 \times$ $130 \mathrm{~mm}$, matrix $160 \times 132$, voxel size $=1.5 \times 1.5 \times 4 \mathrm{~mm}$ ) with and without parallel acquisition (sensitivity encoding, SENSE $=2$ ), resulting in an acquisition time (TA) of 14:06 min and 7:30 min per GRASE scan, respectively. The relatively long TR warrants that T1 weighting is reduced substantially. To include variation related to repositioning, the volunteers went off the scan table after acquiring the first set of GRASE images (with and without parallel acquisition). After repositioning, the second GRASE set (with and without parallel acquisition) was acquired. The order in which the with/without parallel acquisition scans were acquired was switched after half of the volunteers were scanned to prevent a 
bias caused by the scanning order. For one volunteer, also multi-slice GRASE images were acquired with SENSE $=3$ (TA: 5:06 $\mathrm{min}), 4$ (TA: 4:12 $\mathrm{min}$ ) and 8 (TA: 2:44 min) with the same spatial coverage.

\section{Analysis}

\section{Preprocessing}

For each volunteer, the GRASE and T1-weighted data were registered to the first echo image of the acquired first GRASE dataset (before repositioning) using the coregistration algorithm of the statistical parametric mapping (SPM12, https://www.fil.ion.ucl.ac.uk/spm/software/ spm12/) toolkit [19]. The diffusion MRI data was first corrected for head displacement, including B-matrix rotation, and eddy current induced geometric distortions using ExploreDTI v4.8.6 [20]. Subsequently, the DWI images were also registered to the native GRASE space.

A singular value decomposition (SVD) filter [21] was used to reduce noise in the multiecho data, and a Gaussian kernel of $1.1 \mathrm{~mm}$ FWHM served to spatially smooth the GRASE and MSE images.

\section{Regions of Interest}

Whole brain fiber tractography was performed using the diffusion tensor with a uniform seed point of $2 \mathrm{~mm}^{3}$, a step size of $1 \mathrm{~mm}$ and a fractional anisotropy threshold of 0.2 . Next, the fiber bundles of interest, the major and minor forceps, were extracted by selecting only those fibers going through the splenium and genu of the corpus callosum, respectively. Thereafter, the splenium and genu were manually delineated on these fiber bundles. Furthermore, using Freesurfer (version 5.3 [22]), the corpus callosum, all white matter, cortical gray matter, caudate nuclei, thalamus and putamen were automatically segmented from the T1 weighted images. This resulted in 6 white matter regions of interest (ROIs) (major and minor forceps, the genu and splenium of the corpus callosum, the whole corpus callosum and all white matter) and 4 gray matter ROIs (cortical gray matter, caudate nuclei, thalamus and putamen).

\section{Multi-exponential T2 relaxometry analysis}

Multi-exponential analysis of the multi-echo data was performed using the non-negative least squares (NNLS) [9]. To solve the NNLS, a basis set of 120 logarithmically spaced relaxation functions (T2 range 15 to $2000 \mathrm{~ms}$ ) was used. The algorithm was regularized using an additional minimal energy constraint that allows an increased misfit between 2 and $2.5 \%\left(1.020 \leq \chi_{\mathrm{reg}} / \chi_{\min } \leq 1.025\right)$ [23]. Besides the most commonly applied NNLS, the reproducibility of a recently proposed method for myelin-water estimation, the orthogonal 
matching pursuit (OMP) [24], was also assessed. The OMP algorithm was solved by using a basis set of 1000 logarithmically spaced relaxation functions (T2 range 15 to $2000 \mathrm{~ms}$ ) [24].

Due to B1 inhomogeneities and imperfect slice profiles, the T2 relaxation does not behave as a pure exponential decay. To model the decay more accurately, the extended phase graph (EPG) was used to determine the T2 decay based on non-ideal refocusing pulses. The slice profile was estimated based on the Fourier transform of the slice-selective excitation pulse and, subsequently, the basis set was constructed by integrating the EPG decay profiles across the slice [17].

The B1 inhomogeneities were calculated by solving the multi-exponential problem for a range of possible B1 errors and subsequently selecting the B1 error that corresponds to the lowest residual of the fit. Determination of the B1 error is a noise sensitive procedure, therefore, to reduce possible errors due to noisy measurements, the multi-echo data were spatially averaged such that cubic voxels of approximately $4 \mathrm{~mm}^{3}$ were obtained. Subsequently, the resulting B1 error map was smoothed using a Gaussian kernel of $2.3 \mathrm{~mm}$ FWHM. The spatial averaging and kernel size were selected, because the B1 map is expected to have smooth transitions without strong local changes. A B1 error range of 0.5-1.0 was used, which is equivalent to a flip angle range of $90-180^{\circ}$.

The MWF was calculated as the ratio of myelin-water associated T2 components (15-40 $\mathrm{ms})$ to all T2 components (15-2000 ms). Previously, it was reported that the distribution of the MWF is skewed [25], [26]. Here we systematically observed that the MWF distribution in an ROI was (right-)skewed, and therefore we calculated the median to represent the MWF of the ROIs.

\section{Statistical analysis}

First, to investigate the applicability of multi-slice GRASE imaging, the MWF values estimated using the GRASE were plotted against the MWF values obtained with the singleslice MSE reference method. For this comparison, the MWF values estimated from the GRASE scan, obtained in the same session, were used.

Second, the reproducibility of the GRASE without parallel acquisition and the GRASE with an acceleration factor of 2 was evaluated using Bland-Altman (BA) analysis, the coefficient of variation $(\mathrm{CoV})$, the repeatability coefficient $(\mathrm{RC})$ and the intra-class correlation coefficient (ICC) [27].

In the BA analysis, for each ROI the bias between two methods as well as the $95 \%$ limits of agreement (LoA) are determined, where it is expected that these limits include $95 \%$ of the differences between two measurements of the methods. A one-sample t-test was used to test whether the bias between scan sessions was significantly different from zero. Furthermore, BA plots were created to inspect the agreement between the two methods visually. The $\mathrm{CoV}$ was determined as the within-subject standard deviation ( $\left.\mathrm{SD}_{\mathrm{ws}}\right)$ relative to the overall mean for all subjects. The RC was defined as $1.95 \times \sqrt{2} \times S D_{w s}$, and provides a bound for the difference between two measurements for $95 \%$ of the observations. An ICC 
was calculated using a 1-way random model. The ICC can be interpreted as the fraction of total variance to the variance related to biological variation. An ICC of $>.60$ is considered to be good reproducible [28].

Last, the effect of further acceleration was assessed in a single volunteer. The MWF values obtained using the scans without parallel acceleration from the first session are compared to those from the scans with acceleration factors of 2, 3, 4 and 8 from the second session, using a BA analysis.

\section{Image quality}

To estimate the level of noise in the GRASE images with varying acceleration factors, the spatial variation, the signal-to-noise ratio (SNRraw) of the raw images, and the SNR of the multi-exponential fits (SNRfit) were calculated. The spatial variation was defined as the standard deviation of MWF values in the relatively homogeneous splenium. Since a multichannel head coil was used, the calculation of the SNRraw is not straightforward. For such cases, $\mathrm{Wu}$ et al. proposed a general framework for assessing SNRraw, calculating the noise as mean of the background [29]. Therefore, the SNRraw was estimated as the mean signal obtained from a relatively homogeneous region of interest in the splenium (TE $=10 \mathrm{~ms}$ image) divided by the mean signal of the noise, measured in the noise regions of the air. The $\mathrm{SNR}_{\mathrm{fit}}$ was estimated as the mean signal of the splenium (TE $=10 \mathrm{~ms}$ image) divided by the standard deviation of the NNLS fit residuals [30].

\section{Results}

\section{Applicability}

To investigate whether the multi-slice acquisition yields similar results compared to the reference method for MWF estimation, the single-slice reference MWF maps were compared to the corresponding slice in the multi-slice GRASE MWF maps. Due to variations in slice positioning, not all ROIs are available in all of the subjects. In Figure 4.1, the agreement of the GRASE and reference MSE MWF estimation is shown. On average the MWF values reported using the GRASE are approximately higher by $0.9 \%$ absolute MWF values compared to the MSE. Only the MWF in the cortical gray matter is found to be lower using the GRASE. When comparing the MSE to the GRASE with an acceleration factor of 2, a comparable bias of $0.8 \%$ absolute MWF values is observed. 


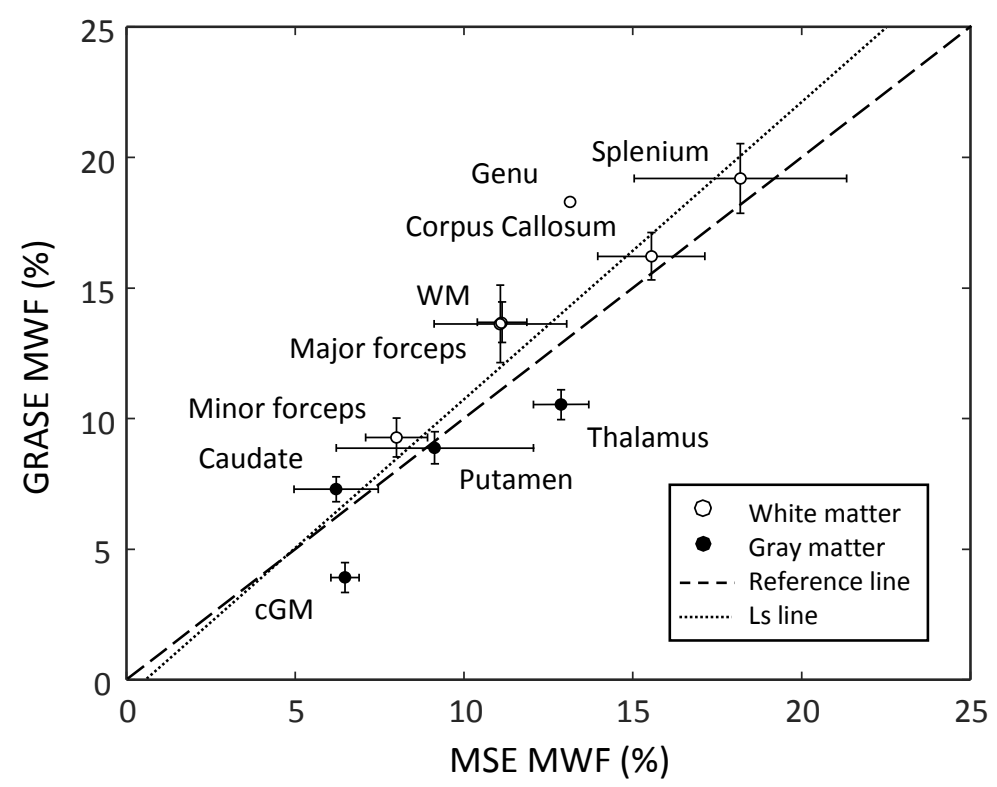

Figure 4.1: The relation of the MWF values obtained using the reference MSE sequence, and the multi-slice GRASE sequence without parallel acceleration. The dashed line represents the line of equality, and the dotted line is a least-squares fit through the data points added for visualization. Note that the Genu is only available in one subject. Error bars depict 1 standard error.

\section{Reproducibility}

The LoA and reproducibility measures of the GRASE MWF estimation, as well as the mean and between-subject standard deviation of the MWF for both measurements, are shown in Table 4.1 for both the NNLS and OMP algorithms and an acceleration factor of 1 , and in Table 4.2 for an acceleration factor of 2. The ICC of the NNLS and OMP results for both acceleration factor 1 and 2 are indicative of a good reproducibility. Only the MWF estimated with the OMP algorithm in the caudate nuclei showed a bias between the measurements that was significantly different from $0(p=.03)$. None of the other measurements had a bias significantly different from $0(p>.05)$.

Furthermore, the BA plots are shown in Figure 4.2 for the NNLS and in Figure 4.3 for the OMP algorithm. The mean LoA for both acceleration factors are added for visualization. 
Table 4.1: Mean and standard deviation of the MWF values in the ROls as well as the reproducibility measures are shown for the first and second GRASE measurement (indicated by 1 and 2) and for both the NNLS and OMP algorithms, performed with an acceleration factor of 1 .

\begin{tabular}{|c|c|c|c|c|c|c|c|c|c|c|c|c|}
\hline \multirow{3}{*}{$\begin{array}{l}\text { Acceleration factor } 1 \\
\text { ROI }\end{array}$} & \multicolumn{6}{|c|}{ NNLS } & \multicolumn{6}{|c|}{ OMP } \\
\hline & \multicolumn{2}{|c|}{ MWF (\%) } & \multirow{2}{*}{ LoA } & \multirow{2}{*}{$\mathrm{RC}$} & \multirow{2}{*}{$\begin{array}{l}\mathrm{CoV} \\
(\%)\end{array}$} & \multirow{2}{*}{ ICC } & \multicolumn{2}{|c|}{ MWF (\%) } & \multirow{2}{*}{ LoA } & \multirow{2}{*}{$\mathrm{RC}$} & \multirow{2}{*}{$\begin{array}{l}\mathrm{CoV} \\
(\%)\end{array}$} & \multirow{2}{*}{ ICC } \\
\hline & 1 & 2 & & & & & 1 & 2 & & & & \\
\hline \multicolumn{13}{|l|}{ White matter } \\
\hline Genu & $14.8 \pm 2.1$ & $14.5 \pm 2.9$ & $-3.9 ; 3.3$ & 3.2 & 6.8 & .94 & $13.5 \pm 1.9$ & $12.3 \pm 3.2$ & $-2.6 ; 4.9$ & 3.6 & 10.1 & .82 \\
\hline Splenium & $20.0 \pm 2.0$ & $19.7 \pm 3.4$ & $-3.1 ; 2.5$ & 2.1 & 3.3 & .87 & $19.3 \pm 2.3$ & $19.0 \pm 3.1$ & $-1.5 ; 2.2$ & 1.7 & 3.1 & .97 \\
\hline Major forceps & $14.0 \pm 1.9$ & $14.4 \pm 2.7$ & $-1.8 ; 2.4$ & 1.3 & 2.9 & .94 & $13.9 \pm 1.9$ & $14.0 \pm 2.5$ & $-1.9 ; 1.8$ & 1.4 & 3.6 & .96 \\
\hline Minor forceps & $10.7 \pm 1.7$ & $10.2 \pm 1.6$ & $-1.2 ; 1.5$ & 1.1 & 3.4 & .96 & $9.6 \pm 1.6$ & $9.7 \pm 1.4$ & $-1.7 ; 1.5$ & 1.4 & 5.2 & .93 \\
\hline $\mathrm{CC}$ & $16.8 \pm 1.7$ & $16.3 \pm 2.2$ & $-2.9 ; 2.0$ & 2.1 & 4.7 & .89 & $16.1 \pm 1.6$ & $15.0 \pm 2.3$ & $-1.9 ; 4.1$ & 3.0 & 7.0 & .76 \\
\hline WM & $13.7 \pm 1.5$ & $13.6 \pm 1.7$ & $-1.8 ; 1.6$ & 1.3 & 3.5 & .93 & $13.1 \pm 1.3$ & $12.8 \pm 1.5$ & $-1.4 ; 2.0$ & 1.5 & 4.1 & .90 \\
\hline \multicolumn{13}{|l|}{ Gray matter } \\
\hline cGM & $5.0 \pm 1.1$ & $5.5 \pm 1.4$ & $-1.3 ; 2.1$ & 1.9 & 10.2 & .82 & $5.4 \pm 1.0$ & $5.4 \pm 1.2$ & $-1.8 ; 1.8$ & 1.3 & 9.0 & .82 \\
\hline Caudate nuclei & $6.9 \pm 1.0$ & $6.8 \pm 1.8$ & $-2.5 ; 2.4$ & 1.9 & 9.9 & .82 & $6.9 \pm 0.9$ & $5.7 \pm 1.4$ & $-0.7 ; 3.1$ & 2.3 & 13.1 & .51 \\
\hline Thalamus & $10.5 \pm 1.5$ & $10.5 \pm 2.0$ & $-2.4 ; 2.4$ & 1.7 & 6.0 & .90 & $11.2 \pm 1.5$ & $10.3 \pm 2.0$ & $-1.8 ; 3.5$ & 2.0 & 6.7 & .80 \\
\hline Putamen & $7.5 \pm 1.1$ & $7.8 \pm 1.7$ & $-2.6 ; 3.1$ & 2.1 & 9.8 & .71 & $8.1 \pm 1.1$ & $8.2 \pm 1.8$ & $-3.0 ; 2.8$ & 1.9 & 8.5 & .72 \\
\hline Mean & & & $-2.4 ; 2.3$ & 1.9 & 6.1 & .88 & & & $-1.8 ; 2.8$ & 2.0 & 7.0 & .82 \\
\hline
\end{tabular}

Abbreviations: NNLS, non-negative least squares; OMP, orthogonal matching pursuit; CC, corpus callosum; WM, white matter; cGM, cortical gray matter; ROI, region of interest; MWF, myelin-water fraction; LoA, limits of agreement; RC, repeatability coefficient; CoV, coefficient of variation; ICC, intra-class correlations coefficient.

Table 4.2: Mean and standard deviation of the MWF values in the ROls as well as the reproducibility measures are shown for the first and second GRASE measurement (indicated by 1 and 2) and for both the NNLS and OMP algorithms, performed with an acceleration factor of 2 .

\begin{tabular}{|c|c|c|c|c|c|c|c|c|c|c|c|c|}
\hline \multirow{3}{*}{$\begin{array}{l}\text { Acceleration factor } 2 \\
\text { ROI }\end{array}$} & \multicolumn{6}{|c|}{ NNNLS } & \multicolumn{6}{|c|}{ OMP } \\
\hline & \multicolumn{2}{|c|}{ MWF (\%) } & \multirow{2}{*}{ LoA } & \multirow{2}{*}{$\mathrm{RC}$} & \multirow{2}{*}{$\begin{array}{l}\mathrm{CoV} \\
(\%)\end{array}$} & \multirow{2}{*}{ ICC } & \multicolumn{2}{|c|}{ MWF (\%) } & \multirow{2}{*}{ LoA } & \multirow{2}{*}{$\mathrm{RC}$} & \multirow{2}{*}{$\begin{array}{l}\mathrm{CoV} \\
(\%)\end{array}$} & \multirow{2}{*}{ ICC } \\
\hline & 1 & 2 & & & & & 1 & 2 & & & & \\
\hline \multicolumn{13}{|l|}{ White matter } \\
\hline Genu & $14.5 \pm 2.9$ & $15.4 \pm 1.5$ & $-3.6 ; 3.6$ & 3.0 & 6.1 & .78 & $13.9 \pm 2.2$ & $13.7 \pm 1.3$ & $-3.2 ; 3.7$ & 2.9 & 7.4 & .72 \\
\hline Splenium & $19.7 \pm 3.4$ & $18.8 \pm 2.4$ & $-2.3 ; 2.6$ & 1.7 & 2.8 & .92 & $18.0 \pm 1.8$ & $18.2 \pm 2.5$ & $-2.7 ; 2.3$ & 1.9 & 3.7 & .92 \\
\hline Major forceps & $14.4 \pm 2.7$ & $14.0 \pm 2.1$ & $-2.7 ; 3.4$ & 1.5 & 3.4 & .94 & $13.0 \pm 1.9$ & $13.8 \pm 2.2$ & $-2.2 ; 0.7$ & 1.4 & 3.9 & .94 \\
\hline Minor forceps & $10.2 \pm 1.6$ & $10.5 \pm 1.1$ & $-0.8 ; 2.2$ & 2.4 & 7.1 & .76 & $9.6 \pm 1.9$ & $10.0 \pm 1.2$ & $-3.2 ; 2.4$ & 2.2 & 8.0 & .77 \\
\hline $\mathrm{CC}$ & $16.3 \pm 2.2$ & $16.7 \pm 2.1$ & $-4.1 ; 4.0$ & 3.1 & 6.8 & .68 & $15.6 \pm 1.7$ & $15.6 \pm 2.2$ & $-3.8 ; 3.8$ & 3.0 & 6.9 & .72 \\
\hline WM & $13.4 \pm 1.4$ & $13.5 \pm 1.3$ & $-2.0 ; 2.1$ & 1.6 & 4.4 & .85 & $12.9 \pm 1.3$ & $12.9 \pm 1.2$ & $-2.1 ; 2.1$ & 1.7 & 4.8 & .82 \\
\hline \multicolumn{13}{|l|}{ Gray matter } \\
\hline cGM & $5.0 \pm 1.0$ & $5.4 \pm 0.7$ & $-2.4 ; 2.6$ & 1.1 & 7.7 & .87 & $5.2 \pm 1.0$ & $5.5 \pm 0.8$ & $-1.7 ; 1.1$ & 1.2 & 8.4 & .80 \\
\hline Caudate nuclei & $7.2 \pm 1.9$ & $7.4 \pm 2.0$ & $-0.8 ; 1.6$ & 1.9 & 9.3 & .85 & $6.7 \pm 1.4$ & $6.2 \pm 2.0$ & $-3.8 ; 4.9$ & 3.8 & 21.5 & .32 \\
\hline Thalamus & $10.0 \pm 1.7$ & $10.2 \pm 1.0$ & $-2.3 ; 2.7$ & 1.9 & 6.7 & .77 & $10.5 \pm 1.8$ & $10.1 \pm 1.3$ & $-2.3 ; 3.2$ & 2.4 & 8.4 & .77 \\
\hline Putamen & $7.3 \pm 1.4$ & $7.5 \pm 1.9$ & $-3.6 ; 3.9$ & 2.6 & 12.6 & .58 & $7.8 \pm 1.5$ & $7.9 \pm 2.0$ & $-4.3 ; 4.1$ & 2.8 & 12.7 & .53 \\
\hline Mean & & & $-2.5 ; 2.9$ & 2.1 & 6.7 & .80 & & & $-2.9 ; 2.8$ & 2.3 & 8.6 & .73 \\
\hline
\end{tabular}

Abbreviations: NNLS, non-negative least squares; OMP, orthogonal matching pursuit; CC, corpus callosum; WM, white matter; cGM, cortical gray matter; ROI, region of interest; MWF, myelin-water fraction; LoA, limits of agreement; RC, repeatability coefficient; CoV, coefficient of variation; ICC, intra-class correlations coefficient. 
A

Acceleration factor 1

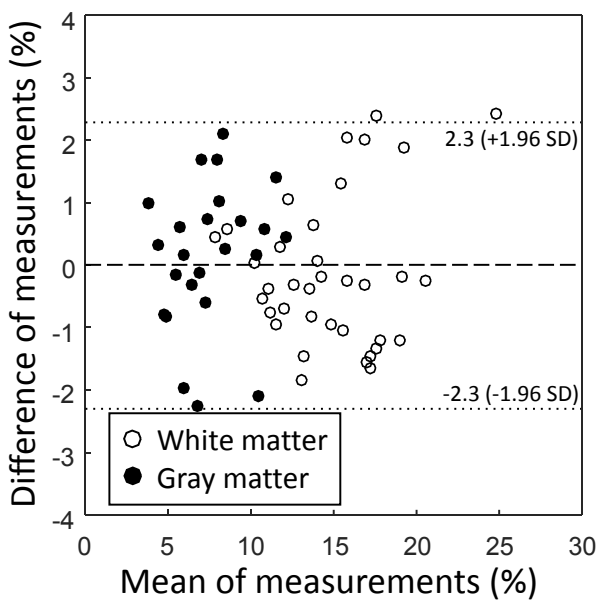

Acceleration factor 2

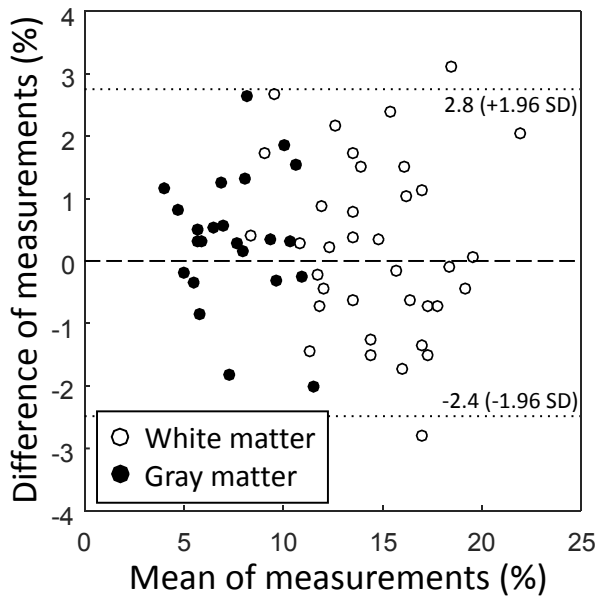

Figure 4.2: Bland-Altman plots for the MWF values estimated using the NNLS algorithm and obtained using acceleration factors 1 (A) and 2 (B). The dashed line represents the x-axis, and the dotted lines shows the mean limits of agreement between the measurements.

A

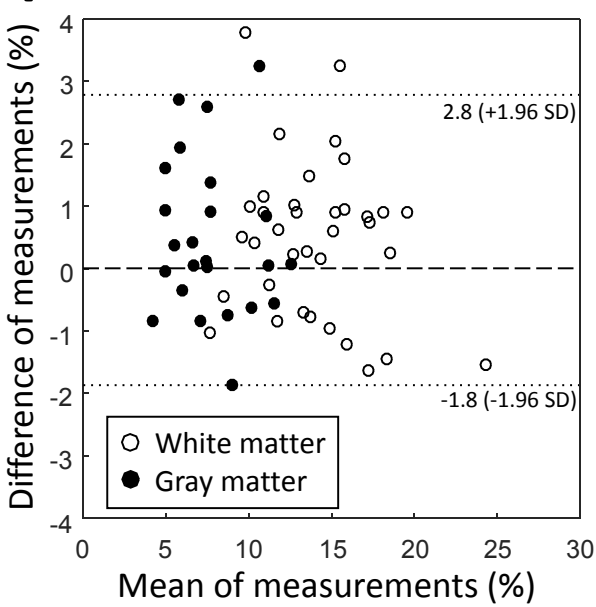

B

Acceleration factor 2

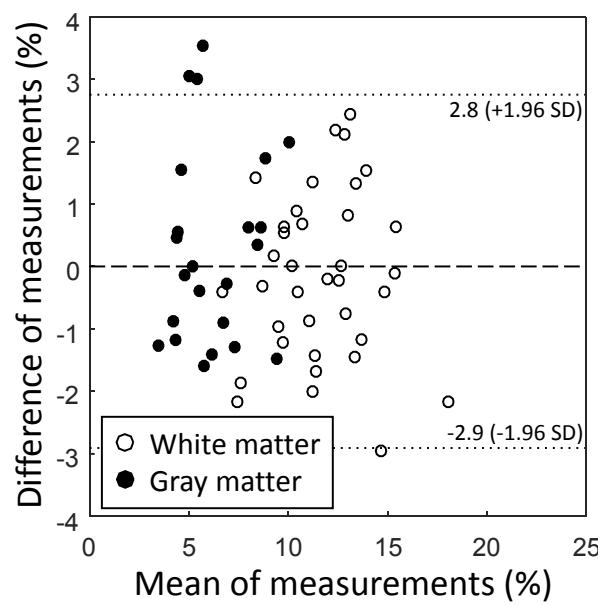

Figure 4.3: Bland-Altman plots for the MWF values estimated using the OMP algorithm and obtained using acceleration factors 1 (A) and 2 (B). The dashed line represents the $x$-axis, and the dotted lines shows the mean limits of agreement between the measurements. 


\section{Image quality}

The MWF maps of one subject are shown in Figure 4.4 for the various acceleration factors. The spatial variation of the MWF values in the splenium increases for increasing acceleration factors (Figure $4.5 \mathrm{~A}$ ). Up to factor 4 , white matter and gray matter can still be distinguished to some extent.

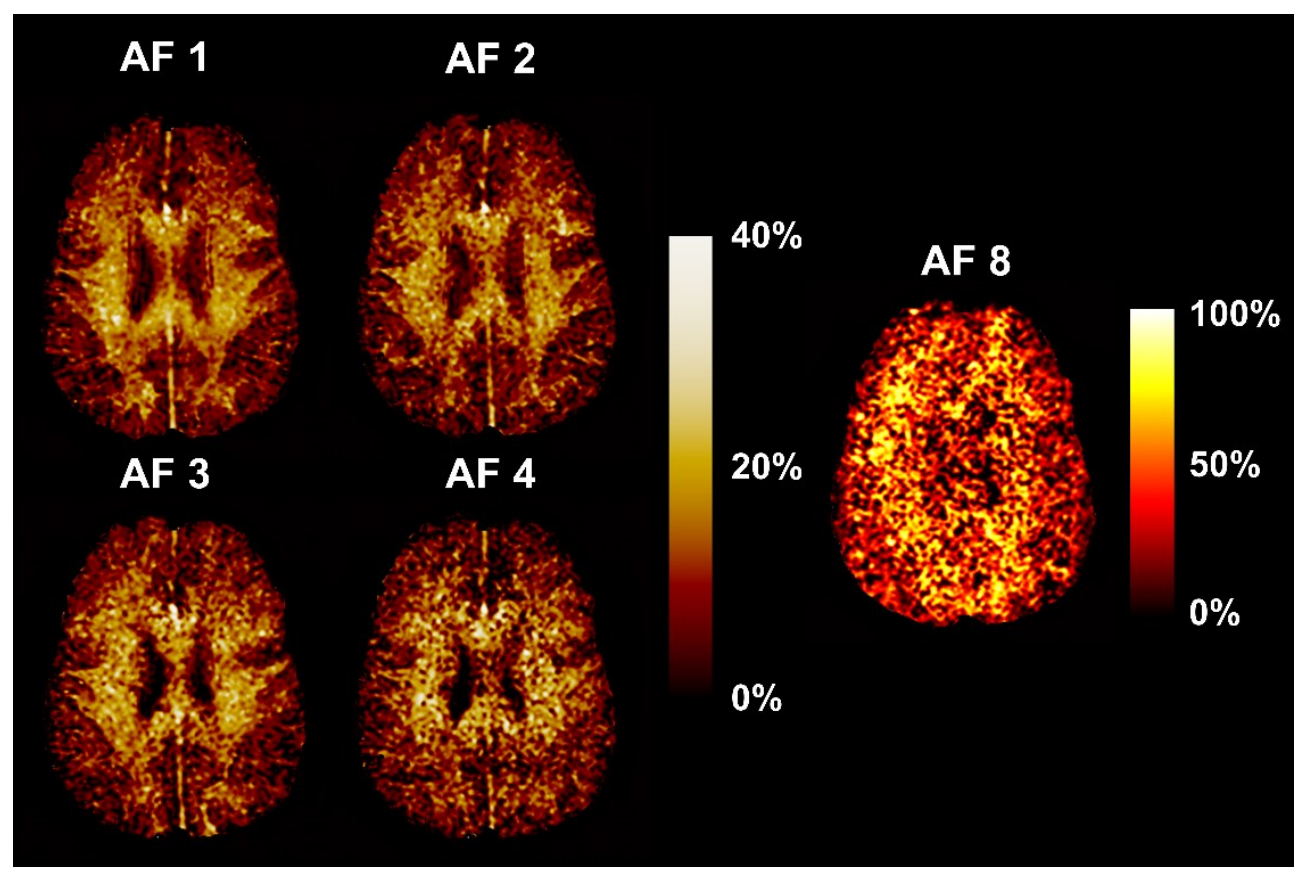

Figure 4.4: Transverse MWF maps estimated from the multi-slice GRASE sequence by the NNLS for varying acceleration factors, 1 up to 8 . Note that the MWF values for acceleration factor 8 ranges from 0 to $100 \%$.

The SNRraw decreased for an increasing acceleration factor (Figure 4.5B). Furthermore, the SNR fit shows a similar decrease for acceleration factors up to 4 (Figure $4.5 \mathrm{C}$ ). Thereafter, for an acceleration factor of 8 , a very steep drop in SNR fit is observed, indicating a steep reduction in the accuracy of the fits.

\section{Further acceleration}

The effect of an increased acceleration factor is shown in Figure 4.6, where the MWF values estimated by the NNLS from scans with varying acceleration factors are shown with respect to no parallel acceleration. The bias and LoA are also plotted in the figures. 

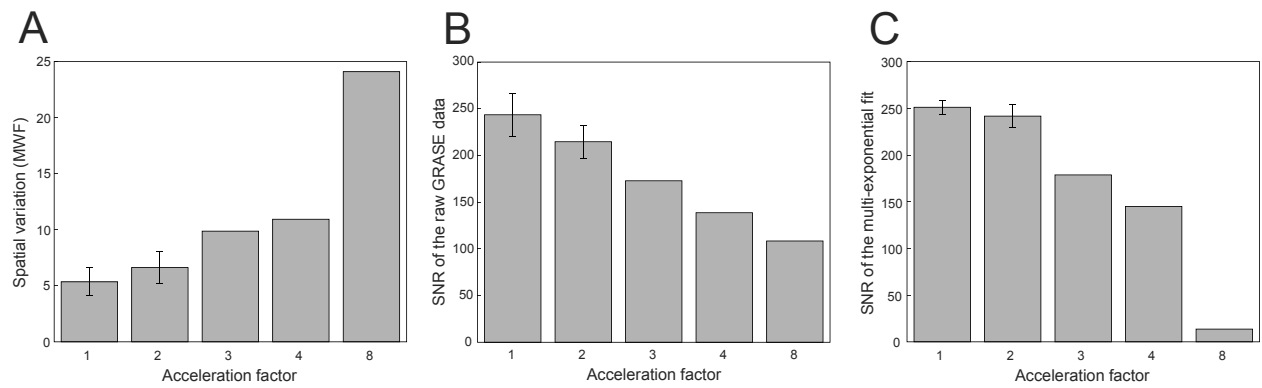

Figure 4.5: Spatial variation of the MWF values in the splenium (A), SNR of the raw GRASE data (B), and SNR of the multi-exponential fits for varying acceleration factors (C)S. Error bars depict 1 standard error. Note that the values shown for acceleration factor 3 up to 8 are obtained in a single subject.

A

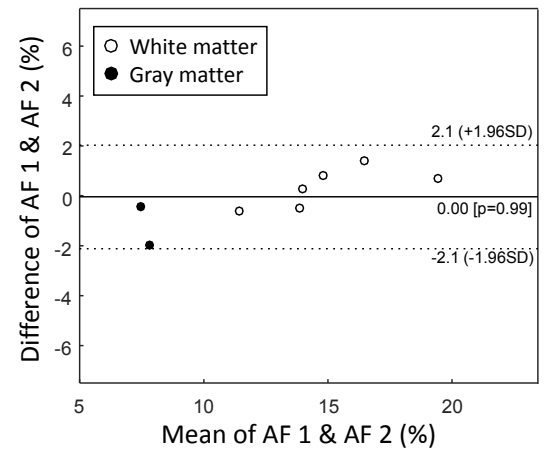

C

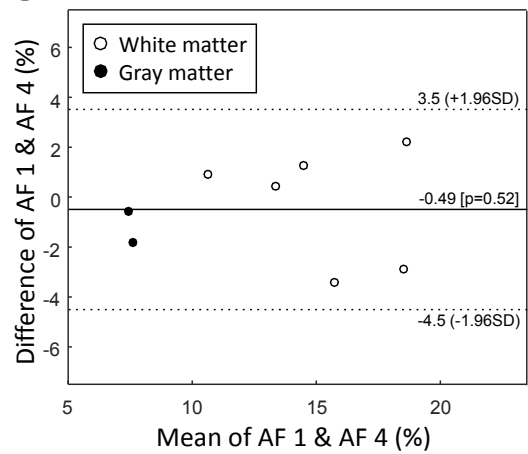

B
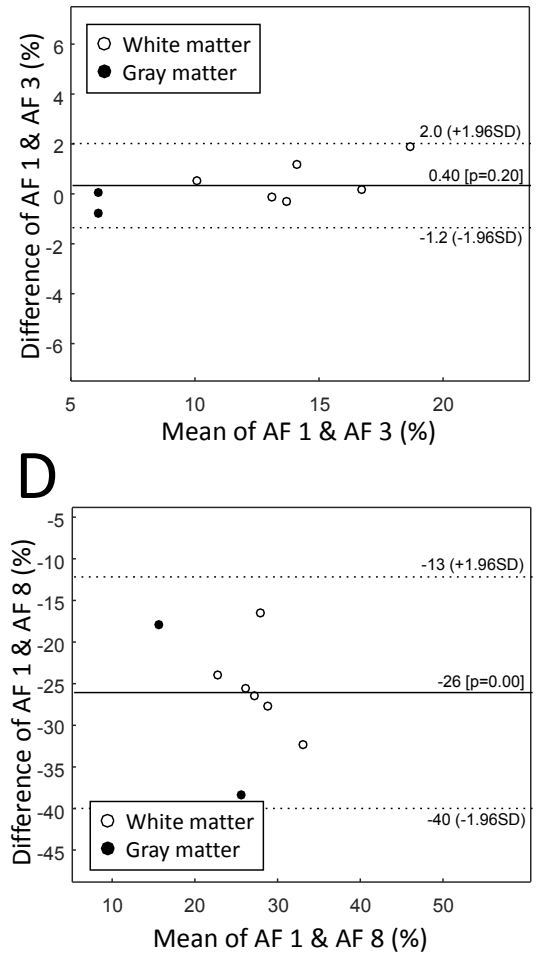

Figure 4.6: Bland-Altman plots of the MWF values obtained using acceleration factor of 2 (A), 3 (B), 4 (C) and 8 (D) plotted versus the MWF values obtained with an acceleration factor of 1 . The solid lines represent the bias between measurements, and the dotted lines shows the limits of agreement between the measurements. 


\section{Discussion}

Initial MWF research has mainly focused on slow single-slice MSE acquisitions. Recently, methods that allow a whole brain coverage in clinically applicable scan times have emerged [12], [31]. Most of these whole brain methods are performed using a 3D acquisition mode. While this prevents distortions from slice-selective excitation pulses, scanning time is often relatively long and subject motion can corrupt the acquired 3D image. In this study, we have shown the applicability and reproducibility of MWF estimation using fast whole brain multi-slice GRASE imaging with post-acquisition slice-profile correction for varying acceleration factors using parallel imaging.

When we consider not only the parallel acceleration techniques but also the image resolution and FOV, the multi-slice GRASE sequence proposed in this study is roughly twice as fast compared to the 2D CPMG sequence proposed by Akhondi-Asl et al. [15], as well as other 3D GRASE methods [12], [32]-[34]. Although, it should be noted that compared to 3D alternatives the coverage of multi-slice GRASE is reduced by $20 \%$ due to the slice gap of $1 \mathrm{~mm}$.

\section{Applicability}

The MWF values obtained with the multi-slice GRASE sequence are generally in good agreement with those estimated with the reference MSE sequence, showing a slight bias between the two methods of at most $0.9 \%$ in absolute MWF. Magnetization transfer effects introduced by the slice-selective excitation pulses likely have an influence on the determination of the MWF [15]. Slice-selective pulses cause off-resonance excitation of bound hydrogen protons (i.e. macromolecules) in neighboring slices. The magnetization of these macromolecules exchanges with the more freely moving proton pool, subsequently decreasing its visibility and the pertaining signal. This effect could have a direct consequence on the MWF, as the myelin content is determined relative to the total signal. A decrease in signal could artificially increase the apparent MWF, resulting in a (slightly) higher MWF in multi-slice GRASE sequences compared to the reference MSE sequence. However, a previous study showed that for MSE, the signal of the myelin-water pool decreases even more due to magnetization transfer effects, subsequently lowering the MWF [35]. Therefore, the exact role of the magnetization transfer effects on the reported bias of the multi-slice GRASE relative to the reference method remains unclear. Previously, two studies investigated the agreement between a 3D GRASE and 3D MSE sequence and showed excellent agreement [12], [36]. Interestingly, in this study the cortical gray matter and thalamus show a higher MWF value using the MSE sequence. This aberrant behavior could be caused by flow artifacts, since it was previously found that the MSE is affected by flow artifacts differently than the 3D GRASE sequence [12]. Further differences between the two methods could be caused by the differences in echo spacing, where the MSE sequence had 
an echo spacing of $12 \mathrm{~ms}$ (the shortest possible on our clinical system), the GRASE sequence that was used here had a shorter, and more ideal, echo spacing of $10 \mathrm{~ms}$.

\section{Reproducibility}

The reproducibility of MWF estimation has a direct implication on the applicability in clinical studies. In the current study, for the NNLS algorithm we found a within-subject CoV of $6.1 \%$ (range $2.9-10.2 \%$ ) and an ICC of .88 (range .71 - .96) for MWF estimation using a multi-slice GRASE sequence without parallel acceleration. Acquisition with an acceleration factor of 2 resulted in comparable values, a within-subject $\mathrm{CoV}$ of $6.7 \%$ (range $2.8-12.6 \%$ ) and an ICC of .80 (range .58 - .94). Previous studies that investigated the MWF reproducibility reported a within-subject $\mathrm{CoV}$ of $19 \%$ (range $7-40 \%$ ) and $12.6 \%$ (range 4.4 $25.1 \%$ ) for a single-slice MSE sequence [37], [38], while a $\mathrm{CoV}$ of $3.99 \%$ (range 1.77-8.93\%) and ICC of .76 were found for a 3D MSE sequence [39]. Furthermore, in a 3D GRASE sequence a $\mathrm{CoV}$ of $13.2 \%$ (range 2.2-55.3\%) (measured in a single subject) [40], and an ICC of .83 (range .79-.88) [41] were previously reported. Hence, the multi-slice GRASE acquisition with acceleration factor 2 still provides a reproducibility that is comparable to or even better than previous studies.

In case of the OMP algorithm slightly worse reproducibility was found, with a withinsubject $\mathrm{CoV}$ of $7.0 \%$ (range $3.1-13.1 \%$ ) and an ICC of .82 (range $.51-.97$ ) for an acceleration factor of 1 , and a within-subject $\mathrm{CoV}$ of $8.6 \%$ (range $3.7-21.5 \%$ ) and an ICC of .73 (range .32 - .94) for an acceleration factor of 2. Especially in the caudate nuclei the OMP showed poor reproducibility. Therefore, the OMP should be used with caution especially in small subcortical regions.

While the reproducibility for the acquisition without parallel acceleration is on average slightly better compared to an acceleration factor of 2, there are no large differences in reproducibility. Therefore, the increased noise due to the parallel acquisition cannot be the determining factor that influences the reproducibility in this study. Instead, other factors such as differences in slice position due to repositioning, the influence of B1 and B0 inhomogeneities, and the noise susceptible multi-exponential analysis could be important factors explaining the variation between the multi-slice scans.

\section{Further acceleration}

As expected, a higher acceleration factor leads to an increased spatial variation and decreased SNRraw and SNR fit. Previously, it was stated that the results from the multiexponential fit become inaccurate when the SNRraw drops below a certain threshold (approximately 100) [42]. The SNR raw for acceleration factors up to 4 sufficiently fulfills this requirement, indicating that there are no major inaccuracies in the multi-exponential analysis. However, it is clearly observed that the LoA with respect to no parallel acceleration 
are wider for acceleration factor 4, indicating a reduced reproducibility. Interestingly, based on the LoA, an acceleration factor of 3 seems to be comparable or even better as an acceleration factor of 2, possibly indicating that these results are still reproducible. However, since these results are obtained in a single subject this conclusion cannot be drawn formally.

\section{Clinical perspectives}

Previous clinical studies investigating white matter myelin abnormalities in multiple sclerosis typically reported a difference of approximately 2 to $5 \%$ in absolute myelin content compared to controls [37], [43], [44]. Here we found that the average RC of the multi-slice GRASE acquisition with acceleration factor up to 2 is about $2 \%$, indicating that such differences in myelin content can be found in $95 \%$ of the observations. Furthermore, the multi-slice acquisition is less susceptible to subject motion compared to 3D acquisition, where motion can corrupt the entire image volume. This is especially relevant in specific subject/patient groups, such as young children or patients with claustrophobic anxiety. While subject motion cannot be prevented with a $2 \mathrm{D}$ acquisition, it does not compromise the entire dataset.

\section{Study considerations}

In this study, the slice-profile was estimated from the slice-selective excitation pulse using a small-tip-angle approximation. While this approximation is generally considered to be a useful approximation of the Bloch equations for small flip angles (up to approximately $90^{\circ}$ ), it is not as accurate as solving the Bloch equations numerically. However, a recent study compared fitting $\mathrm{T} 2$ relaxation signals using the small-tip-angle approximation to numerically solving the Bloch equations, and found that in case of adequate SNRraw (i.e. > 100) and a three-parameter approach (estimating T2 time, amplitude and B1 error) the small-tip-angle approximation can still provide reliable multi-exponential fits [45]. Hence, we chose to employ the much less complex small-tip-angle approximation. 


\section{Conclusions}

In this study, the applicability and reproducibility of multi-slice myelin-water imaging is evaluated. It was shown that the multi-slice GRASE images are in excellent agreement with the single slice reference method for MWF imaging, with a bias of no more than $.9 \%$ in absolute MWF values. Furthermore, the multi-slice GRASE MWF estimation without parallel imaging acceleration and with an acceleration factor of 2 are found to be highly reproducible, with an average ICC of .80 . Hence, a multi-slice GRASE acquisition with parallel acceleration factor 2 and a scan time of 7:30 min still provides an excellent reproducibility. 


\section{References}

[1] K. Nave and H. B. Werner, "Myelination of the Nervous System: Mechanisms and Functions," Annu. Rev. Cell Dev. Biol., vol. 30, pp. 503-533, Oct. 2014.

[2] E. Hutchinson et al., "Children with new-onset epilepsy exhibit diffusion abnormalities in cerebral white matter in the absence of volumetric differences," Epilepsy Res., vol. 88, no. 2-3, pp. 208-214, Feb. 2010.

[3] M. F. Glasser and D. C. Van Essen, "Mapping Human Cortical Areas In Vivo Based on Myelin Content as Revealed by T1- and T2-Weighted MRI," J. Neurosci., vol. 31, no. 32, pp. 11597-11616, Aug. 2011.

[4] M. A. van Buchem et al., "Global estimation of myelination in the developing brain on the basis of magnetization transfer imaging: a preliminary study," Am. J. Neuroradiol., vol. 22, no. 4, pp. 762-6, Apr. 2001.

[5] C. Laule et al., "Magnetic resonance imaging of myelin," Neurotherapeutics, vol. 4, no. 3, pp. 460484, Jul. 2007.

[6] M. N. Uddin, T. D. Figley, R. A. Marrie, and C. R. Figley, “Can T1w/T2w ratio be used as a myelin-specific measure in subcortical structures? Comparisons between FSE-based T1w/T2w ratios, GRASE-based T1w/T2w ratios and multi-echo GRASE-based myelin water fractions," NMR Biomed., vol. 31, no. 3, p. e3868, Mar. 2018.

[7] V. Sheth et al., "Magnetic resonance imaging of myelin using ultrashort Echo time (UTE) pulse sequences: Phantom, specimen, volunteer and multiple sclerosis patient studies," Neuroimage, vol. 136, pp. 37-44, Aug. 2016.

[8] A. C. Seifert, C. Li, M. J. Wilhelm, S. L. Wehrli, and F. W. Wehrli, "Towards quantification of myelin by solid-state MRI of the lipid matrix protons," Neuroimage, vol. 163, pp. 358-367, Dec. 2017.

[9] K. P. Whittall and A. L. MacKay, "Quantitative interpretation of NMR relaxation data," J. Magn. Reson., vol. 84, no. 1, pp. 134-152, Aug. 1989.

[10] S. C. L. Deoni, B. K. Rutt, T. Arun, C. Pierpaoli, and D. K. Jones, "Gleaning multicomponent T1 and T2 information from steady-state imaging data," Magn. Reson. Med., vol. 60, no. 6, pp. 1372 1387, Dec. 2008.

[11] E. Alonso-Ortiz, I. R. Levesque, and G. B. Pike, "MRI-based myelin water imaging: A technical review," Magn. Reson. Med., vol. 73, no. 1, pp. 70-81, Jan. 2015.

[12] T. Prasloski et al., "Rapid whole cerebrum myelin water imaging using a 3D GRASE sequence," Neuroimage, vol. 63, pp. 533-539, Oct. 2012.

[13] J. Guo, Q. Ji, and W. E. Reddick, "Multi-slice myelin water imaging for practical clinical applications at 3.0 T," Magn. Reson. Med., vol. 70, no. 3, pp. 813-822, Sep. 2013.

[14] D. Kumar, S. Siemonsen, C. Heesen, J. Fiehler, and J. Sedlacik, "Noise robust spatially regularized myelin water fraction mapping with the intrinsic B1-error correction based on the linearized version of the extended phase graph model," J. Magn. Reson. Imaging, vol. 43, no. 4, pp. 800-817, Apr. 2016.

[15] A. Akhondi-Asl, O. Afacan, M. Balasubramanian, R. V. Mulkern, and S. K. Warfield, "Fast myelin water fraction estimation using 2D multislice CPMG," Magn. Reson. Med., vol. 76, no. 4, pp. 13011313, Oct. 2016. 
[16] A. Petrovic, E. Scheurer, and R. Stollberger, "Closed-form solution for T2 mapping with nonideal refocusing of slice selective CPMG sequences," Magn. Reson. Med., vol. 73, no. 2, pp. 818-827, Feb. 2015.

[17] R. M. Lebel and A. H. Wilman, "Transverse relaxometry with stimulated echo compensation," Magn. Reson. Med., vol. 64, no. 4, pp. 1005-1014, Jun. 2010.

[18] A. Mackay, K. Whittall, J. Adler, D. Li, D. Paty, and D. Graeb, “In vivo visualization of myelin water in brain by magnetic resonance," Magn. Reson. Med., vol. 31, no. 6, pp. 673-677, Jun. 1994.

[19] K. J. Friston, J. Ashburner, S. Kiebel, T. Nichols, and W. Penny, Eds., Statistical Parametric Mapping. Elsevier, 2007.

[20] A. Leemans, B. Jeurissen, J. Sijbers, and D. Jones, “ExploreDTI: a graphical toolbox for processing, analyzing, and visualizing diffusion MR data," Proc. 17th Sci. Meet. Int. Soc. Magn. Reson. Med., vol. 17, no. 2, p. 3537, 2009.

[21] M. Bydder and J. Du, "Noise reduction in multiple-echo data sets using singular value decomposition," Magn. Reson. Imaging, vol. 24, no. 7, pp. 849-856, Sep. 2006.

[22] B. Fischl and A. M. Dale, "Measuring the thickness of the human cerebral cortex from magnetic resonance images," Proc. Natl. Acad. Sci., vol. 97, no. 20, pp. 11050-11055, Sep. 2000.

[23] M. G. Skinner, S. H. Kolind, and A. L. MacKay, "The effect of varying echo spacing within a multiecho acquisition: better characterization of long T2 components," Magn. Reson. Imaging, vol. 25, no. 6, pp. 840-847, Jul. 2007.

[24] G. S. Drenthen, W. H. Backes, A. P. Aldenkamp, G. J. Op 't Veld, and J. F. A. Jansen, "A new analysis approach for T2 relaxometry myelin water quantification: Orthogonal Matching Pursuit," Magn. Reson. Med., vol. 81, no. 5, pp. 3292-3303, Nov. 2018.

[25] M. Dayan et al., "MRI Analysis of White Matter Myelin Water Content in Multiple Sclerosis: A Novel Approach Applied to Finding Correlates of Cortical Thinning," Front. Neurosci., vol. 11, p. 284, 2017.

[26] S. Kolind, L. Matthews, H. Johansen-berg, M. I. Leite, and C. R. Steven, "Myelin Water Imaging Reflects Clinical Variability in Multiple Sclerosis," Neuroimage, vol. 60, pp. 263-270, 2012.

[27] S. M. Wong et al., "On the Reproducibility of Inversion Recovery Intravoxel Incoherent Motion Imaging in Cerebrovascular Disease," Am. J. Neuroradiol., vol. 39, no. 2, pp. 226-231, Feb. 2018.

[28] D. V. Cicchetti, "Guidelines, criteria, and rules of thumb for evaluating normed and standardized assessment instruments in psychology.," Psychol. Assess., vol. 6, no. 4, pp. 284-290, 1994.

[29] B. Wu, C. Wang, Y. Pang, and X. Zhang, "Comparison of SNR Calculation Methods for in vivo Imaging," in Proc. Intl. Soc. Mag. Reson. Med., 2010, vol. 18, p. 3153.

[30] E. L. Macmillan et al., "Myelin water and T2 relaxation measurements in the healthy cervical spinal cord at 3.0T: Repeatability and changes with age," Neuroimage, vol. 54, pp. 1083-1090, 2011.

[31] T. D. Nguyen et al., "Feasibility and reproducibility of whole brain myelin water mapping in 4 minutes using fast acquisition with spiral trajectory and adiabatic T2prep (FAST-T2) at 3T," Magn. Reson. Med., vol. 76, no. 2, pp. 456-465, Aug. 2016.

[32] T. D. Faizy et al., "Age-Related Measurements of the Myelin Water Fraction derived from 3D multi-echo GRASE reflect Myelin Content of the Cerebral White Matter," Sci. Rep., vol. 8, no. 1, p. 14991, 2018.

[33] S. Ocklenburg et al., "Myelin Water Fraction Imaging Reveals Hemispheric Asymmetries in Human White Matter That Are Associated with Genetic Variation in PLP1," Mol. Neurobiol., vol. 56, no. 6, pp. 3999-4012, 2019. 
[34] N. Uddin, T. D. Figley, and C. R. Figley, "Effect of echo time and T2-weighting on GRASE-based T1w/T2w ratio measurements at 3T," Magn. Reson. Imaging, vol. 51, pp. 35-43, 2018.

[35] I. M. Vavasour, K. P. Whittall, D. K. B. Li, and A. L. Mackay, "Different Magnetization Transfer Effects Exhibited by the Short and Long T2 Components in Human Brain," Magn. Reson. Med., vol. 44, pp. 860-866, 2000.

[36] E. Ljungberg et al., "Rapid myelin water imaging in human cervical spinal cord," Magn. Reson. Med., vol. 78, no. 4, pp. 1482-1487, Oct. 2017.

[37] I. M. Vavasour, C. M. Clark, D. K. B. Li, and A. L. MacKay, "Reproducibility and reliability of MR measurements in white matter: Clinical implications," Neuroimage, vol. 32, pp. 637-642, Aug. 2006.

[38] I. R. Levesque, C. L. L. Chia, and G. B. Pike, "Reproducibility of in vivo magnetic resonance imaging-based measurement of myelin water," J. Magn. Reson. Imaging, vol. 32, no. 1, pp. 60-68, Jun. 2010.

[39] S. M. Meyers et al., "Multicenter measurements of myelin water fraction and geometric mean T2: Intra- and intersite reproducibility," J. Magn. Reson. Imaging, vol. 38, no. 6, pp. 1445-1453, Dec. 2013.

[40] S. M. Meyers, S. H. Kolind, and A. L. MacKay, "Simultaneous measurement of total water content and myelin water fraction in brain at $3 \mathrm{~T}$ using a T2 relaxation based method," Magn. Reson. Imaging, vol. 37, pp. 187-194, Apr. 2017.

[41] M. Arshad, J. A. Stanley, and N. Raz, "Test-retest reliability and concurrent validity of in vivo myelin content indices: Myelin water fraction and calibrated T1w/T2w image ratio," Hum. Brain Mapp., vol. 38, no. 4, pp. 1780-1790, Apr. 2017.

[42] S. M. Meyers et al., "Reproducibility of myelin water fraction analysis: a comparison of region of interest and voxel-based analysis methods," Magn. Reson. Imaging, vol. 27, no. 8, pp. 1096-1103, Oct. 2009.

[43] W. S. Vargas et al., "Measuring longitudinal myelin water fraction in new multiple sclerosis lesions," NeuroImage Clin., vol. 9, pp. 369-375, 2015.

[44] C. Laule et al., "Water content and myelin water fraction in multiple sclerosis," J. Neurol., vol. 251, pp. 284-293, Mar. 2004.

[45] K. C. McPhee and A. H. Wilman, "Transverse relaxation and flip angle mapping: Evaluation of simultaneous and independent methods using multiple spin echoes," Magn. Reson. Med., vol. 77, no. 5, pp. 2057-2065, 2017. 



\section{Chapter 5}

\section{Lower myelin-water content of the frontal lobe in childhood absence epilepsy}

Gerhard S. Drenthen*, Eric L.A. Fonseca Wald*, Walter H. Backes, Mariette H.J.A. Debeij-van Hall, Jos G.M. Hendriksen, Albert P. Aldenkamp, R. Jeroen Vermeulen, Sylvia Klinkenberg, Jacobus F.A. Jansen

*Authors contributed equally

Epilepsia 2019; 60(8):1689-1696. DOI: 10.1111/epi.16280. 


\section{Abstract}

The frontal lobe in childhood absence epilepsy (CAE) might be affected due to the suggested involvement of the frontal lobe during absence seizures, and reports on attentional deficits. Previously, subtle white matter abnormalities have been reported in CAE. However, the impact of one of the most characteristic components of the white matter, the myelin content, remains underdetermined. Therefore, this study investigated whether the myelin content in frontal areas is adversely affected in CAE compared to controls.

Seventeen children with childhood absence epilepsy (aged $9.2 \pm 2.1$ years) and 15 age and sex-matched controls (aged $9.8 \pm 1.8$ years) underwent neuropsychological assessment and an MRI exam. T2 relaxometry scans were used to distinguish myelin-water from tissue water and to determine the myelin-water fraction (MWF) in the frontal, temporal, parietal, occipital, and insular lobes. A linear regression model including age and sex as covariates was used to investigate group differences. Furthermore, the relationship of MWF with cognitive performance and epilepsy characteristics was determined.

The frontal lobe revealed a significantly lower myelin-water content in children with CAE compared to controls over the developmental age range of $6-12$ years (5.7 $\pm 1.0 \%$ vs. 6.6 $\pm 1.1 \%, \mathrm{p}=.02$ ). This association was not found for any of the other four lobes ( $\mathrm{p}>.10)$. No significant relation was found between myelin-water content and cognitive performance or epilepsy characteristics. The lower frontal myelin-water content of children with CAE in comparison to healthy controls probably reflects an altered neurodevelopmental aspect in CAE, of which the underlying mechanisms still need to be unraveled. 


\section{Introduction}

Childhood absence epilepsy (CAE) is characterized by seizures of brief losses of awareness occurring multiple times per day, in otherwise normally developing school-aged children. Ictal activity on an electroencephalogram (EEG) shows approximately $3 \mathrm{~Hz}$ generalized spike-and-wave discharges (GSWD). The generation or propagation of these GSWD were previously suggested to have a frontal involvement, although the precise identification of brain regions involved is an ongoing debate [1], [2]. MRI of the brain is normally omitted from the standard clinical work-up as specific structural brain abnormalities are not regarded to be part of this generalized epilepsy syndrome. Nevertheless, cognitive [3] and neuroimaging [4]-[7] studies in children with absence epilepsy have raised concerns on the true neurocognitive impact of this allegedly benign syndrome. A higher incidence of academic difficulties and neurocognitive deficits, mainly attentional deficits, for which the frontal lobe plays an important role, have been observed in CAE [8]-[16]. Furthermore, a study employing diffusion MRI reported altered structural connectivity in children with CAE, which was suggested to imply abnormal myelination [17]. However, while diffusion MRI can provide markers sensitive to myelin content, these markers are not specific to the myelin content only [18].

The process of myelination is most active during the first two years of life, but continues through adulthood [19]. Myelin is wrapped around the axons causing efficient action potential conduction and increased conductance velocity in axons, which is essential for information transmission through neural circuits [20]. Abnormal and exceptional synchronization of neural activity (e.g. epileptic seizures), may be related to the myelination process and thus neurodevelopment in children with CAE. Currently, quantitative data on in-vivo myelin content and its change during brain development is lacking in CAE imaging studies.

The myelin content can be quantified through MR imaging of myelin-water. Myelinwater quantification is based on the quantification of water between the bilayers of the myelin sheath using T2 relaxometry. Since water is an important component of myelin, making up to $40 \%$ of the total myelin volume, myelin-water imaging can be regarded as a specific marker of myelin content [21]. Water trapped between the bilayers of the myelin has a faster T2 relaxation rate compared to more freely moving water in the intra- and extracellular spaces. Myelin content can be quantified using the fraction of myelin-water signal to the total water signal, the so-called myelin-water fraction (MWF).

In this study, myelination was investigated in children with CAE and controls, by applying MWF imaging. Due to the frontal involvement during seizures in children with CAE, we hypothesize altered myelin content in frontal areas.

The primary aim of the current study is to assess differences in myelin-water content between children with CAE and controls and the secondary aim is to explore the 
relationship of myelin-water content with the duration of CAE and neurocognitive performance.

\section{Methods}

\section{Inclusion}

Children with CAE were prospectively recruited via pediatricians and pediatric neurologists within the adherence areas of Kempenhaeghe Epilepsy Centre, a tertiary epilepsy center. Children with a clinical diagnosis of CAE were included based on the following criteria: 1) Primarily presenting with daily occurring episodes of brief loss of consciousness in an otherwise normal child and an EEG showing ictal $3 \mathrm{~Hz}(2.5-4.5 \mathrm{~Hz})$ generalized rhythmic spike-and-wave complexes with a discharge duration of at least 3 seconds on a present or former EEG (in accordance to ILAE statements for CAE [22], [23]). 2) Early absence epilepsy, defined as a confirmed diagnosis or seizure onset within 2 years. 3) Six to twelve years of age. Healthy age and sex-matched controls were recruited via advertisements. To be included, controls needed to follow regular school without major problems. All caregivers and children aged 12 years old gave written permission prior to inclusion in the study. This research protocol was approved by the medical ethics committee azM/UM NL55455.068.15/METC152055 and is listed at clinicaltrials.gov under NCT02954107.

\section{Clinical and neuropsychological assessment}

Clinical characteristics including age of onset, seizure semiology, duration of epilepsy, drughistory, EEG-reports were collected in an online data management system (Castor Electronic Data Capture, CIWIT bv, The Netherlands). All children underwent a multidisciplinary assessment, which included a medical assessment, a 24h-video-EEG, and a neuropsychological assessment. Six subtests of the WISC-III (similarities, vocabulary, picture completion, coding, block design, and symbol search) were used to assess general intelligence and processing speed [24], [25]. The Bourdon-Vos, a paper and pencil cancelation test, was used to test for sustained visual attention and vigilance [26]. Based on age-dependent normative data, the WISC-III and Bourdon-Vos were expressed as standard scores. 


\section{MRI Acquisition}

All subjects were scanned on a 3 Tesla unit (Philips Achieva, Best, the Netherlands) using a 32-element phased array coil. To minimize moving during the MRI, children were carefully prepared beforehand using a combination of video and written information. Parents were also instructed to practice lying still with the child at home, and to be present with them in the magnet room during the scanning. First, for anatomical reference and segmentation, T1weighted $3 \mathrm{D}$ turbo field echo images were acquired (repetition time $(\mathrm{TR})=8.36 \mathrm{~ms}$, echo time $(\mathrm{TE})=3.84 \mathrm{~ms}$, flip angle $(\mathrm{FA})=8^{\circ}$, voxel size $=1 \mathrm{~mm}^{3}$ ). For MWF estimation, whole cerebrum 2D multi-slice gradient and spin echo (GRASE) images were acquired (TR $=3000$ $\mathrm{ms}$, 32 echoes with $9.3 \mathrm{~ms}$ echo spacing, range 9.3-297.6 ms, EPI factor $=3$, Turbo factor $=32$, 24 slices, $1 \mathrm{~mm}$ slice gap, field of view $=228 \times 180 \times 119 \mathrm{~mm}$, matrix $152 \times 120$, voxel size = $1.5 \times 1.5 \times 4 \mathrm{~mm}$ ) with parallel acquisition (sensitivity encoding, SENSE = 2), scan duration = 5:45 minutes [27].

\section{Analysis}

\section{Preprocessing}

For each subject, the T1 images were registered to the first echo image of the GRASE scans using the statistical parametric mapping (SPM12) toolkit [28]. A singular value decomposition filter was used to reduce noise in the multi-echo data [29], and a Gaussian kernel of $2.4 \mathrm{~mm}$ full width at half maximum was used to spatially smooth the GRASE images. Furthermore, all images were visually checked for motion artifacts. Some slices with severe motion artifacts were removed from subsequent analyses.

\section{ROIs}

The white matter was parcellated into five lobes: the frontal, temporal, parietal, occipital and insular lobes (Figure 5.1). First, the white matter was parcellated using the T1-weighted images employing Freesurfer (version 5.1) [30] based on the 68 cortical regions in the Desikan-Killiany atlas [31] by assigning each white matter voxel to the most proximal cortical region. Finally, the ROIs were slightly eroded to cope with potential partial-volume errors occurring during the co-registration. The volumes of the segmented white matter lobes are shown in Table 5.1. 


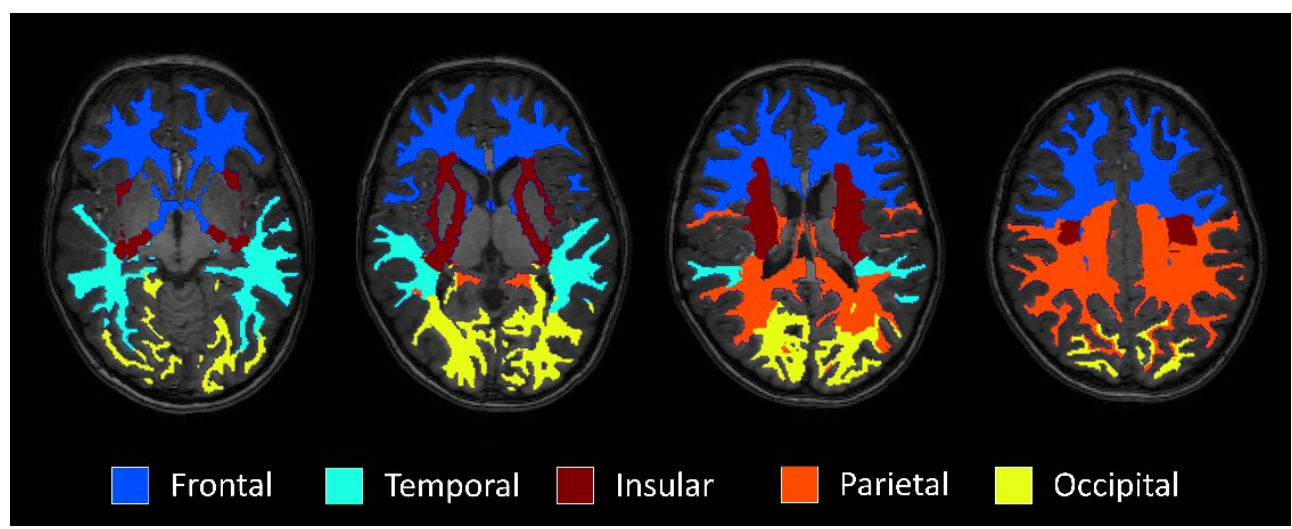

Figure 5.1: The five WM ROls used in this study, representing the frontal, parietal, temporal, occipital and insular lobes.

Table 5.1. WM volumes of the 5 lobes.

\begin{tabular}{llll}
\hline & \multicolumn{2}{l}{ WM volume } & p-value \\
\cline { 2 - 4 } & CAE $(\mathrm{cm} 3)$ & Controls $(\mathrm{cm} 3)$ & .97 \\
\hline Frontal & $165 \pm 29$ & $165 \pm 22$ & .78 \\
Parietal & $119 \pm 19$ & $121 \pm 16$ & .90 \\
Occipital & $41 \pm 7$ & $41 \pm 4$ & .86 \\
Temporal & $64 \pm 12$ & $63 \pm 8$ & .60 \\
Insular & $32 \pm 6$ & $31 \pm 4$ & .99 \\
Whole brain & $421 \pm 51$ & $422 \pm 70$ & \\
\hline
\end{tabular}

Abbreviations: WM, white matter; CAE, childhood absence epilepsy.

\section{Multi-exponential analysis}

Multi-exponential analysis of the multi-echo signal decay data was performed using the orthogonal matching pursuit (OMP) algorithm [32]. To solve the OMP, a basis set of 1000 logarithmically spaced relaxation functions (T2 range 15 to $2000 \mathrm{~ms}$ ) was used. Previously, it was shown that the algorithm provides reproducible measures of myelin-water content in the white matter regions (Chapter 4).

The extended phase graph (EPG) algorithm [33] along with the Fourier transform of the slice-selective excitation pulse [34] were used to account for possible stimulated echoes caused by B1 inhomogeneities, and imperfect slice profiles due to slice-selective excitation [27].

The OMP results in a T2 spectrum, indicating which $\mathrm{T} 2$ components are present in the signal. The water trapped between the bilayers of the myelin is known to decay with a relatively fast T2 time of 15 to $40 \mathrm{~ms}$. Therefore, the MWF was calculated as the ratio of 
myelin-water associated T2 components (15-40 ms) to all T2 components (15-2000 ms) [21], [33], [35]. Since the MWF distribution in an ROI is (right-)skewed, we applied the median to represent the MWF in the ROIs.

\section{Statistical Analysis}

Differences between children with CAE and controls in age, general intelligence, processing speed, and the speed and accuracy of the Bourdon-Vos test were assessed using the Student's $t$ test for independent samples. Differences in sex and handedness were assessed with the Chi-square test of independence.

To investigate whether lobar myelin-water content differed between groups a MANCOVA analysis was performed, where the MWF of the five lobes were used as dependent variables. Since myelin varies with age [36] and was previously shown to differ between males and females [37], age and sex are added as covariates in the MANCOVA model. Subsequently, post-hoc linear multivariable regression analyses were performed for each lobe to assess differences between groups, correcting for age and sex.

The impact of the duration of CAE and the duration of anti-epileptic drug (AED) use on the frontal myelin-water content was evaluated using a linear multivariable regression model for the CAE group, correcting for age and sex. Furthermore, to explore the relation of frontal myelin-water content with neurocognitive performance, a linear multivariable regression model was used for both groups combined, correcting for age and sex. This multivariable regression model was used for each of the neurocognitive performance variables, general intelligence, and processing speed from the WISC-III, as well as the speed and accuracy of the Bourdon-Vos.

Statistical significance was inferred when $p<.05$.

\section{Results}

\section{Subject characteristics}

The population consisted of 17 children with CAE and 15 controls. The subject characteristics are shown in Table 5.2. The two groups did not differ significantly regarding age, sex, and handedness. All included children were following regular education, except one child included in the CAE group. Nine were taking Ethosuximide (range 14.7 - 27.2 $\mathrm{mg} / \mathrm{kg}$ ), three were taking Valproïc Acid (range $13.3-30.8 \mathrm{mg} / \mathrm{kg}$ ), two were taking Ethosuximide + Valproïc Acid (1.31.8 mg/kg ETM and $22.7 \mathrm{mg} / \mathrm{kg}$ VPA; $2.25 .7 \mathrm{mg} / \mathrm{kg}$ ETM and $14.12 \mathrm{mg} / \mathrm{kg} \mathrm{VPA})$ and one was taking Lamotrigine + Clobazam $(1.4+0.42 \mathrm{mg} / \mathrm{kg})$. At the time of the MRI, two children were still drug-naïve, whereas 15 were on anti-epileptic drug treatment. Processing speed index was significantly lower for children with CAE compared to controls, although both groups were still within normal clinical range. Mean 
performance in the WISC subtests and Bourdon-Vos did not differ significantly between groups. A trend towards worse performance in CAE was present in the WISC subtests and speed for sustained attention in the Bourdon-Vos compared to controls.

Table 5.2. Subject characteristics of children with CAE and controls

\begin{tabular}{llll}
\hline Demographics & CAE & Controls & p-value \\
\hline No & 17 & 15 & - \\
Age (years, mean \pm SD) & $9.2 \pm 2.1$ & $9.8 \pm 1.8$ & .37 \\
Sex (M/F) & $12 / 5$ & $11 / 4$ & .86 \\
Handedness (R/L) & $16 / 1$ & $11 / 4$ & .11 \\
Age of onset (years, mean \pm SD) & $8.0 \pm 2.0$ & - & - \\
Duration of epilepsy (years, mean \pm SD) & $1.2 \pm 0.7$ & - & - \\
Total duration AED use (weeks) & $27 \pm 26$ & - & - \\
Schooling (regular/special) & $16 / 1$ & $15 / 0$ & .34 \\
Family history of epilepsy (Yes/No) & $2 / 15$ & $1 / 14$ & .62 \\
\hline Neurocognitive performance & & & .09 \\
\hline WISC & & & .03 \\
$\quad$ Subtests (mean \pm SD) & $9.6 \pm 1.9$ & $10.9 \pm 2.3$ & \\
$\quad$ Processing speed index (mean & $95 \pm 15$ & $108 \pm 14$ & .09 \\
$\quad$ SD) & & & .38 \\
Bourdon-Vos & & & \\
$\quad$ Speed (mean z-score \pm SD) & $-0.9 \pm 0.8$ & $-0.3 \pm 1.2$ & \\
$\quad$ Speed (z-score $\leq-1 /$ z-score $>-1)$ & $12 / 17$ & $-0.3 \pm 0.7$ & \\
$\quad$ Accuracy (mean z-score \pm SD) & $0.0 \pm 0.9$ & $4 / 15$ & \\
$\quad$ Accuracy (z-score $\leq-1 / z-s c o r e>-1)$ & $5 / 17$ & & \\
\hline
\end{tabular}

Abbreviations: AED, Anti-epileptic drug; CAE, Childhood Absence Epilepsy; No, number; L, left; R, right; $\mathrm{M}$, male; F, female; WISC, Wechsler Intelligence Scale for Children; SD, standard deviation.

\section{Myelin content}

A T1-weighted scan with the corresponding MWF map of a child with CAE is shown in Figure 5.2. 


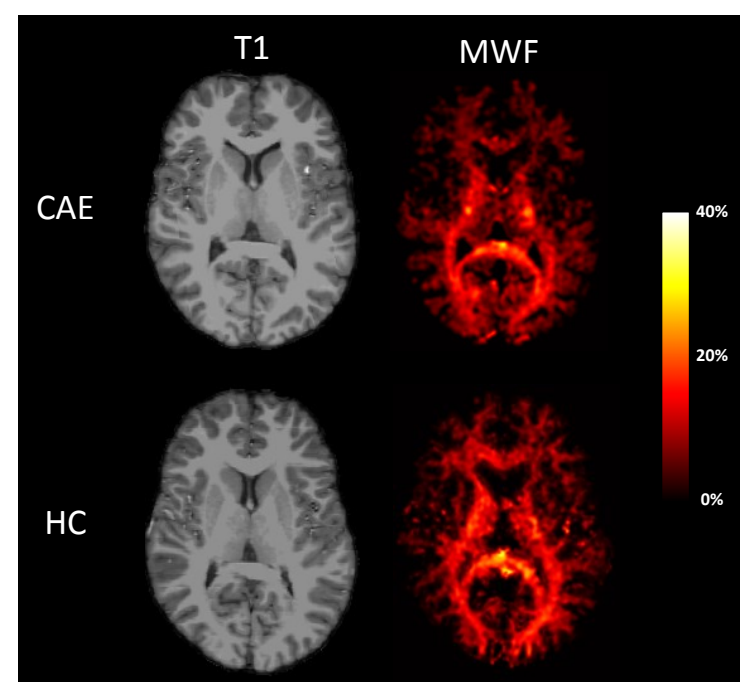

Figure 5.2: An axial slice of a T1-weighted image and myelin-water fraction map for a 10-year-old boy with CAE and a 10-year-old male healthy control, positioned through the splenium and genu of the corpus callosum.

The MANCOVA analysis revealed that the lobar myelin-water content is significantly different between children with CAE and controls, while controlling for effects of age and sex $(\mathrm{p}<.05)$. Post-hoc regression analysis showed a significantly lower frontal myelin-water content in children with CAE compared to controls. For any of the other four lobes no significant differences were found (Table 5.3). A scatter plot of the frontal MWF values as a function of age is shown in Figure 5.3. Least-square lines for CAE (solid) and controls (dashed) are added for visualization, qualitatively revealing that the frontal myelin-water content increases with age. Moreover, the difference between children with CAE and controls does not vary over age.

Table 5.3. MWF values in the 5 lobes for children with CAE and controls. Note that the raw MWFvalues are shown, and that the effects of age and sex are not included. The $p$-value denotes the significance level of the group difference obtained with the regression model.

\begin{tabular}{llll}
\hline MWF Lobes & CAE $(\%)$ & Controls $(\%)$ & p-value \\
\hline Frontal & $7.5 \pm 1.1$ & $8.5 \pm 1.1$ & .02 \\
Parietal & $9.3 \pm 1.2$ & $9.9 \pm 1.1$ & .22 \\
Occipital & $9.2 \pm 1.2$ & $9.7 \pm 1.2$ & .38 \\
Temporal & $6.5 \pm 0.8$ & $7.0 \pm 0.7$ & .09 \\
Insular & $10.3 \pm 1.7$ & $11.1 \pm 1.8$ & .26 \\
\hline
\end{tabular}

Abbreviations: MWF, myelin-water fraction; CAE, childhood absence epilepsy. 


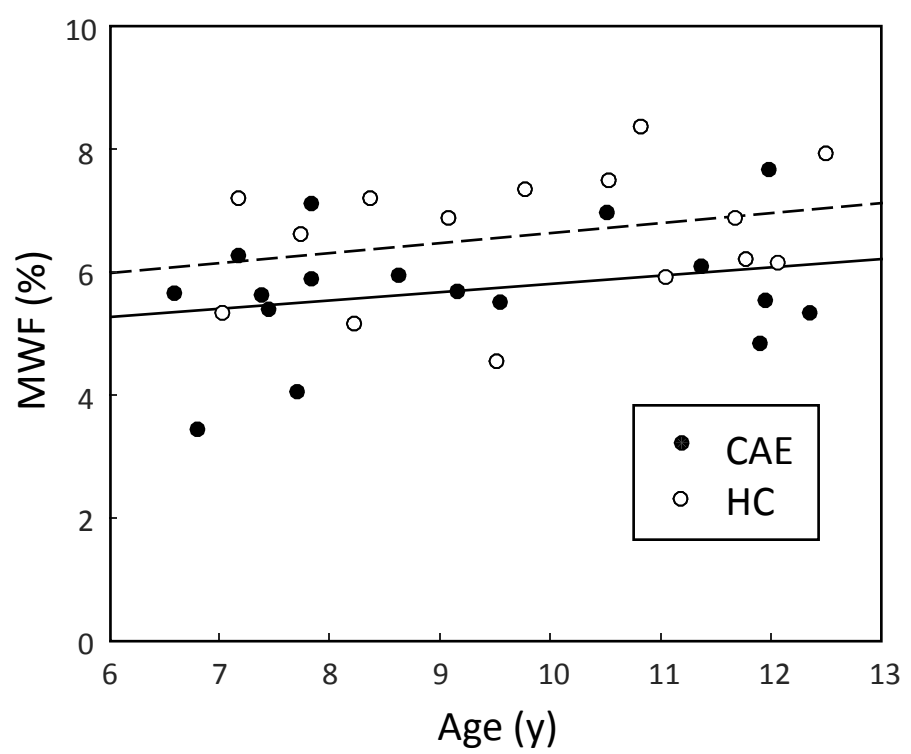

Figure 5.3: Frontal myelin-water fraction as a function of age. Least-square lines for childhood absence epilepsy (solid) and controls (dashed) are added for visualization, qualitatively revealing that the frontal myelin-water content increases with age. Abbreviations used: MWF, myelin-water fraction; CAE, childhood absence epilepsy; $\mathrm{HC}$, healthy controls; $y$, years.

The deepest white matter regions (insular lobe) show the highest myelin-water content, while the myelin-water content in the frontal and temporal lobes are lower compared to the parietal and occipital lobes. These findings are consistent with the known patterns of myelin development, moving from posterior to anterior areas, and outwards from the central to the peripherally regions [38].

Using regression, signification relations were neither found between frontal myelinwater content and the duration of $\operatorname{CAE}(\beta=.13, p=.65)$ nor the duration of AED use $(\beta=-$ $.14, p=.56)$. In the whole sample, no significant relations were found between frontal myelin-water content and general intelligence $(\beta=.24, p=.16)$, the processing speed index ( $\beta$ $=.25, p=.13)$, and the speed $(\beta=-.20, p=.38)$ and accuracy $(\beta=-.15, p=.41)$ of the BourdonVos.

\section{Discussion}

In the current study, the cerebral myelin-water content of children with early onset CAE was investigated and compared with age- and sex-matched controls. A lower myelin-water content was found in the frontal lobe of children with CAE compared to controls, while myelin-water content did not differ in any of the other lobes. Neurocognitive performance, 
and sustained attention in our cohort of children with CAE did not relate significantly to myelin-water content, though the results were indicative of a better performance for increased myelin-water content.

Previously, CAE studies using diffusion MRI reported lower fractional anisotropy (FA) values in the genu, an important part of the corpus callosum in the frontal lobe [6], [39]. However, while diffusion MRI measures are valuable in white matter research, they fail to provide more specific information on the myelin content and cannot discriminate between axonal damage, alterations in myelin content or topographic deviations [40]. Previously, Spader et al. already showed the feasibility of identifying in vivo myelin abnormalities using MRI and reported a reduced MWF in three children with epilepsy [41]. However, it has not been previously employed in a group with generalized epilepsy.

The main function of myelin is accelerating information processing due to the propagation of electrical signals (i.e. action potentials) using saltatory conduction. However, the precise mechanisms are yet to be completely understood.

The observed lower frontal myelin-water content might occur in an early stage of the neurodevelopment (i.e. related to a genetic component), or might be acquired during or after epilepsy onset (i.e. related to epileptiform activity during the vulnerable years of childhood). Though no definite association for the duration of epilepsy was found in this study, the observed abnormality can be interpreted as a neurodevelopmental aspect of the disorder as the maturation of the frontal lobe is still ongoing [42]. Human and animal studies have suggested frontal involvement during the generation or propagation of generalized GSWD, although the exact brain regions involved are of ongoing debate [1], [2]. EEG-fMRI studies have found different patterns of activation [2] and animal studies point to the somatosensory cortex in the generation of GSWDs. On the other hand, deactivation of frontal areas during GSWDs [43] and/or differences in resting state networks involved in attention have also been reported [2] and might be related to changes in myelin content as well. From this relatively small cross-sectional sample we cannot affirm whether the reduced myelin-water content predates the onset of absence epilepsy, or whether the difference in myelin-water content worsens or improves with a longer follow-up time. These aspects need to be elucidated in longitudinal studies.

We observed a lower performance for processing speed index and a trend towards lower general intelligence and speed for sustained attention in children with CAE. This is in agreement with previous studies on neurocognition in CAE [9], [12], [44]. Though no definite association between neurocognition and myelin-water was found in the current study, it has been suggested that myelin may be important in optimizing the timing of information through neural circuits [20]. Evidence also suggests that myelin may be influenced by seizures, as has been shown in preclinical studies where epilepsy induced rats show a reduced myelin content [45]-[47]. Moreover, histopathological studies in focal epilepsy have shown abnormalities in white matter myelination, axonal integrity, and cellular composition [48], [49], although limited data exists in generalized epilepsy as it is 
more difficult to collect tissue samples. Therefore, the used methods and findings of this study are promising for epilepsy research, as it may help understand the relationship of epileptiform activity and neurocognitive co-morbidities better.

\section{Study considerations}

Our study has several important strengths. The study used well defined inclusion criteria for children with CAE in agreement with current ILAE standards. Moreover, the age and sex-matched controls enabled us to make reliable group-level comparisons. Furthermore, to minimize the influence of subject motion, which is an inherent problem when scanning young children, we employed a 2D multi-slice acquisition instead of the more commonly applied 3D acquisition [27]. While 2D imaging does not prevent subject motion artifacts completely, it does allow for removal of corrupted slices, whereas subject motion in 3D acquisition affects the entire volume. In this study fewer than $4 \%$ of the total slices were corrupted, and subsequently removed. The main limitation of this study is the sample size, which limits the power for more in-depth correlation analysis with clinical/epilepsy variables. we did not find a relation between Furthermore, it cannot be excluded that the use of AED's has had a bearing on the results of this study, as Ethosuximide and Valproïc acid have been associated with neurocognitive side effects [9], [50]. Nonetheless, as current guidelines prescribe the use of these AED's, the results in this study reflect children with $\mathrm{CAE}$ at this point in time. Furthermore, no relation was found between the duration of AED use and the frontal myelin-water content in this study, indicating that our results are not driven by medication use. Future studies, preferably, larger populations followed over time, are necessary to elucidate on the relationship between AED use and the myelination process.

\section{Conclusion}

This study, for the first time, found a lower myelin-water content in the frontal lobe in children with CAE compared with age and sex-matched controls, probably implicating an altered neurodevelopmental aspect in CAE. Whether the altered myelin-water content predates the onset of absence epilepsy, or whether the myelin-water content worsens over time cannot be concluded from this study. Therefore, more myelin-specific studies with longitudinal designs are warranted to further investigate the relation of (frontal) myelin content and CAE. 


\section{References}

[1] P. W. Carney, R. A. J. Masterton, D. Flanagan, S. F. Berkovic, and G. D. Jackson, “The frontal lobe in absence epilepsy," Neurology, vol. 78, no. 15, pp. 1157-1165, Apr. 2012.

[2] F. Moeller et al., "Absence seizures: Individual patterns revealed by EEG-fMRI," Epilepsia, vol. 51, no. 10, pp. 2000-2010, 2010.

[3] A. Verrotti, S. Matricardi, V. E. Rinaldi, G. Prezioso, and G. Coppola, "Neuropsychological impairment in childhood absence epilepsy: Review of the literature," J. Neurol. Sci., vol. 359, no. 12, pp. 59-66, 2015.

[4] R. Caplan et al., "Frontal and temporal volumes in Childhood Absence Epilepsy," Epilepsia, vol. 50, no. 11, pp. 2466-2472, 2009.

[5] G. Di Wang et al., "Grey matter anomalies in drug-naïve childhood absence epilepsy: A voxelbased morphometry study with MRI at 3.0 T," Epilepsy Res., vol. 124, pp. 63-66, 2016.

[6] J. S. Liang et al., "Microstructural Changes in Absence Seizure Children: A Diffusion Tensor Magnetic Resonance Imaging Study," Pediatr. Neonatol., vol. 57, no. 4, pp. 318-325, 2016.

[7] K. Xue et al., "Diffusion tensor tractography reveals disrupted structural connectivity in childhood absence epilepsy," Epilepsy Res., vol. 108, no. 1, pp. 125-138, Jan. 2014.

[8] D. Cheng, X. Yan, Z. Gao, K. Xu, X. Zhou, and Q. Chen, "Common and Distinctive Patterns of Cognitive Dysfunction in Children With Benign Epilepsy Syndromes," Pediatr. Neurol., vol. 72, pp. 36-41, Jul. 2017.

[9] D. Masur et al., "Pretreatment cognitive deficits and treatment effects on attention in childhood absence epilepsy," Neurology, vol. 81, no. 18, pp. 1572-1580, 2013.

[10] C. L. Kernan et al., "Neurocognitive profiles in children with epilepsy," Epilepsia, vol. 53, no. 12, pp. 2156-2163, 2012.

[11] C. Cerminara et al., "Attention impairment in childhood absence epilepsy: An impulsivity problem?," Epilepsy Behav., vol. 27, no. 2, pp. 337-341, 2013.

[12] E. D'Agati, C. Cerminara, L. Casarelli, M. Pitzianti, and P. Curatolo, "Attention and executive functions profile in childhood absence epilepsy," Brain Dev., vol. 34, no. 10, pp. 812-817, 2012.

[13] M. Levav, A. F. Mirsky, J. Herault, L. Xiong, N. Amir, and E. Andermann, "Familial Association of Neuropsychological Traits in Patients With Generalized and Partial Seizure Disorders," J. Clin. Exp. Neuropsychol., vol. 24, no. 3, pp. 311-326, May 2002.

[14] A. T. Berg, S. R. Levy, F. M. Testa, and H. Blumenfeld, “Long-term seizure remission in childhood absence epilepsy: Might initial treatment matter?," Epilepsia, vol. 55, no. 4, pp. 551-557, 2014.

[15] K. J. Oostrom, A. Smeets-Schouten, C. L. J. J. Kruitwagen, A. C. B. Peters, and A. JennekensSchinkel, "Not Only a Matter of Epilepsy: Early Problems of Cognition and Behavior in Children With 'Epilepsy Only'--A Prospective, Longitudinal, Controlled Study Starting at Diagnosis," Pediatrics, vol. 112, no. 6, pp. 1338-1344, 2003.

[16] A. Covanis, K. Skiadas, N. Loli, C. Lada, and V. Theodorou, "Absence epilepsy: early prognostic signs," Seizure Eur. J. Epilepsy, vol. 1, no. 4, pp. 281-289, 1992.

[17] W. Qiu et al., "Structural Abnormalities in Childhood Absence Epilepsy: Voxel-Based Analysis Using Diffusion Tensor Imaging," Front. Hum. Neurosci., vol. 10, p. 483, 2016.

[18] C. Laule et al., "Magnetic resonance imaging of myelin," Neurotherapeutics, vol. 4, no. 3, pp. 460484, Jul. 2007.

[19] M. Baraban, S. Mensch, and D. A. Lyons, "Adaptive myelination from fish to man," Brain Res., vol. 1641, pp. 149-161, 2016. 
[20] R. D. Fields, "A new mechanism of nervous system plasticity: Activity-dependent myelination," Nat. Rev. Neurosci., vol. 16, no. 12, pp. 756-767, 2015.

[21] E. Alonso-Ortiz, I. R. Levesque, and G. B. Pike, "MRI-based myelin water imaging: A technical review," Magn. Reson. Med., vol. 73, no. 1, pp. 70-81, Jan. 2015.

[22] A. T. Berg et al., "Revised terminology and concepts for organization of seizures and epilepsies: Report of the ILAE Commission on Classification and Terminology, 2005-2009," Epilepsia, vol. 51, no. 4, pp. 676-685, 2010.

[23] R. S. Fisher et al., "Operational classification of seizure types by the International League Against Epilepsy: Position Paper of the ILAE Commission for Classification and Terminology," Epilepsia, vol. 58, no. 4, pp. 522-530, 2017.

[24] D. Wechsler, Manual for the Wechsler Intelligence Scale for Children, 3rd ed. San Antonio, TX: The Psychological Corporation, 1991.

[25] W. Kort et al., "WISC-III NL. Handleiding en verantwoording. Londen: The Psychological Corporation," 2005.

[26] P. G. Vos, "Bourdon-Vos test handleiding. Swets test Services," 1988.

[27] G. S. Drenthen, W. H. Backes, A. P. Aldenkamp, and J. F. A. Jansen, "Applicability and reproducibility of $2 \mathrm{D}$ multi-slice GRASE myelin water fraction with varying acquisition acceleration," Neuroimage, vol. 195, pp. 333-339, 2019.

[28] K. J. Friston, J. Ashburner, S. Kiebel, T. Nichols, and W. Penny, Eds., Statistical Parametric Mapping. Elsevier, 2007.

[29] M. Bydder and J. Du, "Noise reduction in multiple-echo data sets using singular value decomposition," Magn. Reson. Imaging, vol. 24, no. 7, pp. 849-856, Sep. 2006.

[30] B. Fischl and A. M. Dale, "Measuring the thickness of the human cerebral cortex from magnetic resonance images," Proc. Natl. Acad. Sci., vol. 97, no. 20, pp. 11050-11055, Sep. 2000.

[31] R. S. Desikan et al., "An automated labeling system for subdividing the human cerebral cortex on MRI scans into gyral based regions of interest," Neuroimage, vol. 31, pp. 968-980, Jul. 2006.

[32] G. S. Drenthen, W. H. Backes, A. P. Aldenkamp, G. J. Op 't Veld, and J. F. A. Jansen, "A new analysis approach for T2 relaxometry myelin water quantification: Orthogonal Matching Pursuit," Magn. Reson. Med., vol. 81, no. 5, pp. 3292-3303, Nov. 2018.

[33] T. Prasloski, B. Mädler, Q. S. Xiang, A. MacKay, and C. Jones, "Applications of stimulated echo correction to multicomponent T2 analysis," Magn. Reson. Med., vol. 67, no. 6, pp. 1803-1814, 2012.

[34] R. M. Lebel and A. H. Wilman, "Transverse relaxometry with stimulated echo compensation," Magn. Reson. Med., vol. 64, no. 4, pp. 1005-1014, Jun. 2010.

[35] K. P. Whittall and A. L. MacKay, "Quantitative interpretation of NMR relaxation data," J. Magn. Reson., vol. 84, no. 1, pp. 134-152, Aug. 1989.

[36] E. Kavroulakis et al., "Myelin content changes in probable Alzheimer's disease and mild cognitive impairment: Associations with age and severity of neuropsychiatric impairment," J. Magn. Reson. Imaging, vol. 47, no. 5, pp. 1359-1372, 2018.

[37] M. Arshad, J. A. Stanley, and N. Raz, "Adult age differences in subcortical myelin content are consistent with protracted myelination and unrelated to diffusion tensor imaging indices," Neuroimage, vol. 143, pp. 26-39, Dec. 2016.

[38] K. M. Welker and A. Patton, "Assessment of Normal Myelination with Magnetic Resonance Imaging," Semin. Neurol., vol. 32, pp. 15-28, 2012. 
[39] D. G. Corrêa et al., "Evaluation of deep gray matter volume, cortical thickness and white matter integrity in patients with typical absence epilepsy: a study using voxelwise-based techniques," Neuroradiology, vol. 59, no. 3, pp. 237-245, 2017.

[40] H. Chahboune, A. M. Mishra, M. N. Desalvo, L. H. Staib, M. Purcaro, and D. Scheinost, “DTI abnormalities in anterior corpus callosum of rats with spike-wave epilepsy," Neuroimage, vol. 47, pp. 459-466, 2009.

[41] H. S. Spader et al., "Advances in myelin imaging with potential clinical application to pediatric imaging," Neurosurg. Focus, vol. 34, no. 4, p. E9, Apr. 2013.

[42] S. Määttä et al., "Maturation changes the excitability and effective connectivity of the frontal lobe: A developmental TMS-EEG study," Hum. Brain Mapp., vol. 40, no. 8, pp. 2320-2335, 2019.

[43] F. Moeller, U. Stephani, and M. Siniatchkin, "Simultaneous EEG and fMRI recordings (EEG-fMRI) in children with epilepsy," Epilepsia, vol. 54, no. 6, pp. 971-982, 2013.

[44] A. F. Lopes et al., "Intellectual functioning in children with epilepsy: Frontal lobe epilepsy, childhood absence epilepsy and benign epilepsy with centro-temporal spikes," Seizure, vol. 22, no. 10, pp. 886-892, Dec. 2013.

[45] Y. Luo et al., "Alterations in hippocampal myelin and oligodendrocyte precursor cells during epileptogenesis," Brain Res., vol. 1627, pp. 154-164, 2015.

[46] Y. You et al., "Myelin damage of hippocampus and cerebral cortex in rat pentylenetetrazol model," Brain Res., vol. 1381, pp. 208-216, 2011.

[47] Y. Ye et al., "Altered hippocampal myelinated fiber integrity in a lithium-pilocarpine model of temporal lobe epilepsy: A histopathological and stereological investigation," Brain Res., vol. 1522, pp. 76-87, 2013.

[48] X. Hu et al., "The relationship between the occurrence of intractable epilepsy with glial cells and myelin sheath - an experimental study.," Eur. Rev. Med. Pharmacol. Sci., vol. 20, no. 21, pp. 45164524, 2016.

[49] F. Deleo, M. Thom, L. Concha, A. Bernasconi, B. C. Bernhardt, and N. Bernasconi, "Histological and MRI markers of white matter damage in focal epilepsy," Epilepsy Res., vol. 140, pp. 29-38, 2018.

[50] D. M. Ijff et al., “The Cognitive Profile of Ethosuximide in Children," Pediatr. Drugs, vol. 18, no. 5, pp. 379-385, 2016. 



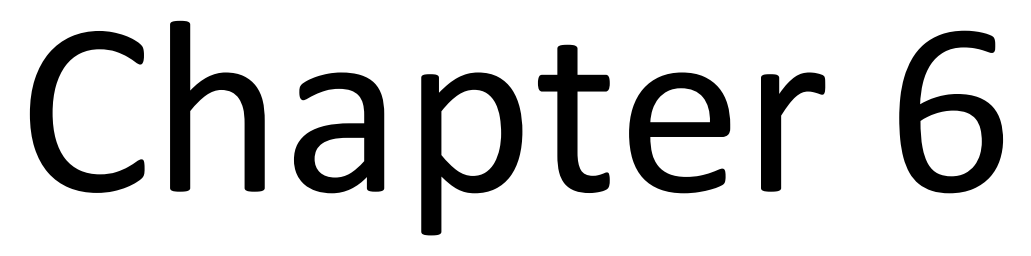

\section{Constructing an axonal-specific myelin developmental atlas and its application to childhood absence epilepsy}

Gerhard S. Drenthen, Eric L.A. Fonseca Wald, Walter H. Backes, Albert P. Aldenkamp, R. Jeroen Vermeulen, Mariette Debeij-van Hall,

Sylvia Klinkenberg, Jacobus F.A. Jansen 


\section{Abstract}

The process of myelination starts in utero around 20 weeks of gestation and continues through adulthood. We first set out to characterize the maturation of the tract-specific myelin content in healthy subjects from childhood (7-12y) into adulthood (18-32y). Second, we apply the resulting normative atlas of development to children with childhood absence epilepsy (CAE), a pediatric epilepsy which was previously characterized by changes in myelin content.

In a prospective cross-sectional study, fifteen healthy children (aged 7-12y), 14 healthy adult participants (aged 18-32y) as well as 17 children with a clinical diagnosis of CAE (aged $6-12 y$ ) were included. For each participant, diffusion weighted images were acquired to reconstruct bundles of white matter tracts and multi-echo multi-slice GRASE images were acquired for myelin-water estimation. Subsequently, a tract-specific myelin development atlas was constructed using the percentual difference in myelin-water content from childhood (12y) to adulthood (25y). The atlas revealed myelination patterns, where tracts in the central regions myelinate prior to peripheral tracts and intra-hemispheric tracts as well as tracts in the left hemisphere myelinate prior to inter-hemispheric tracts and tracts in the right hemisphere, respectively.

No significant differences were found in myelin-water content between children with $\mathrm{CAE}$ and healthy children for neither the early developing tracts $(\beta=-.15, \mathrm{p}=.49)$, nor the tracts that develop in a later stage $(\beta=.26, p=.20)$. However, the difference between the myelin-water of late and early developing tracts is significantly smaller in the children with CAE $(\beta=-.58, \mathrm{p}<.01)$, indicating that CAE is associated with widespread neurodevelopmental myelin differences. 


\section{Introduction}

Human brain development is a complex process that starts in the third gestational week and continues into adulthood. An important structural component for healthy neuronal maturation of the white matter is the myelin sheath. The myelin sheath is an electrically insulating material that is wrapped around the axons allowing fast and efficient conduction of electrical signals in the central nervous system. The process of myelination is most active during the first two years of life, but continues well through adulthood [1]. During healthy development myelin develops in specific patterns [2], generally proceeding from inward to outward regions, and in the posterior to anterior direction [3]. Abnormalities in the myelin content are associated with several neurological and neuropsychiatric disorders such as Alzheimer's disease [4], multiple sclerosis [5], epilepsy [6], Parkinson's disease [7] and schizophrenia [8].

The organization of the human brain can be regarded as a complex network, where local clusters of neurons (grey matter) are inter-connected via long-range axons (white matter). Bundles of axons in the white matter can be reconstructed using diffusion tensor imaging (DTI), which enables the characterization of the structural brain network in terms of white matter tracts that interconnect remote grey matter regions. Previously, tract-specific increases of Fractional Anisotropy (FA) were reported during childhood and adolescence [9]. The FA is often used as a marker of myelin content, however, it is not specific for myelin. While the myelin content does indeed affect the FA to some extent, FA is also driven by the density and diameter of axons, fiber orientations and changes to membrane permeability [10].

Water is an important component of the myelin, making up to $40 \%$ of the total myelin volume. Therefore, myelin-water imaging (MWI) has been extensively applied to estimate the in vivo myelin content. Due to motion restrictions at the microscopic level, the T2 signal arising from water that is trapped between the myelin sheath decays more quickly compared to more freely moving water in the intra- and extra-cellular spaces. Using T2 relaxometry and multi-exponential analysis the different water components can be decomposed from the total signal, and the myelin-water fraction can be obtained as the fraction of myelin-water signal over the total signal (i.e. MWF) [11].

Combining the structural information from DTI with information on the myelin content from MWI might provide a more comprehensive description of the white matter organization in the brain, compared to only the DTI-derived tract trajectories. Previously, it was already shown that myelin-water maps possess distinct patterns along DTI-derived tracts [12], [13]. Ideally, it should be feasible to follow the myelin development of various white matter tracts over time, and differentiate which tracts reflect abnormalities during healthy development. While neurological disorders such as multiple sclerosis show severe disruption of the myelin content, other more subtle and developmental changes might play a role in paediatric epilepsies at various time-points of development. For example, a lower 
frontal myelin content was reported in children with childhood absence epilepsy (CAE) [6], a paediatric epilepsy that is characterized by brief losses of awareness in otherwise normally developing school-aged children. The combined information of DTI and MWI might prove useful in characterizing such subtler changes in myelin content to specific white matter tracts, which may exhibit different development trajectories.

In the current prospective cross-sectional study, we first set out to characterize the maturation of the tract-specific myelin content in healthy subjects from childhood through adolescence into adulthood and subsequently construct a normative atlas of white matter tract myelination. Second, we investigate whether tracts that show different maturation trajectories in healthy controls during adolescence (i.e. early and late developing tracts) show abnormalities in myelin content in children with CAE compared to healthy controls.

\section{Materials and Methods}

\section{Participants}

Fifteen healthy children (aged 7-12y), all following regular school without major problems and 14 healthy adult participants (aged 18-32y) were included in a prospective crosssectional study. Additionally, seventeen children with a clinical diagnosis of CAE (aged 6$12 y$ ) were included, the inclusion criteria were reported previously [6]. Inclusion of healthy children and children with CAE and adults was approved by the medical ethics committee. All caregivers and participants aged $\geq 12$ years old gave written permission prior to inclusion in the study.

The acquired MR images were visually inspected for motion artifacts. In total, 5 children ( 1 healthy control and 4 with CAE) and none of the adults had severe motion artifacts and were removed from subsequent analysis. After exclusion, 14 healthy adults, 14 healthy children and 13 children with CAE remained for subsequent analysis. The subject characteristics are shown in Table 6.1. The healthy children and children with CAE did not differ in age and sex.

Table 6.1: Subject characteristics

\begin{tabular}{lllll}
\hline & Adults & \multicolumn{2}{l}{ Children } & \\
\cline { 3 - 5 } & & Controls & CAE & $p$-value \\
\hline Number \# & 14 & 14 & 13 & - \\
Age (years, mean \pm SD) & $28 \pm 3.4$ & $9.8 \pm 1.9$ & $9.0 \pm 2.0$ & .26 \\
Sex $(\mathrm{M} / \mathrm{F})$ & $8 / 6$ & $11 / 3$ & $9 / 4$ & .58 \\
Age of onset $($ years, mean $\pm \mathrm{SD})$ & - & - & $7.9 \pm 2.0$ & \\
\hline
\end{tabular}

Abbreviations: $\mathrm{CAE}$, childhood absence epilepsy; SD, standard deviation; $M$, male; F, female. 


\section{MRI Acquisition}

All participants were scanned on a 3 Tesla MRI unit (Philips Achieva, Best, the Netherland) using a 32-element phased array coil.

\section{CAE and healthy children dataset}

First, for anatomical reference and segmentation, T1-weighted 3D fast gradient echo images were acquired (repetition time $(\mathrm{TR})=8.36 \mathrm{~ms}$, echo time $(\mathrm{TE})=3.84 \mathrm{~ms}$, flip angle $(\mathrm{FA})=8^{\circ}$, cubic voxel size $=1 \mathrm{~mm}$ ). For MWF estimation, whole cerebrum $2 \mathrm{D}$ multi-slice gradient and spin echo (GRASE) images were acquired (TR $=3000 \mathrm{~ms}, 32$ echoes with $9.3 \mathrm{~ms}$ echo spacing, range 9.3-297.6 ms, EPI factor $=3$, Turbo factor $=32$, 24 slices, $1 \mathrm{~mm}$ slice gap, field of view $=228 \times 180 \times 119 \mathrm{~mm}$, acquisition matrix $152 \times 120$, voxel size $=1.5 \times 1.5 \times 4 \mathrm{~mm}$ ) with parallel acquisition (sensitivity encoding, SENSE $=2$ ) and a scan duration of 5:45 minutes. To reconstruct the tracts in the white matter, diffusion weighted images were acquired $\left(\mathrm{TR}=6579 \mathrm{~ms}, \mathrm{TE}=74 \mathrm{~ms}\right.$, cubic voxel size $=2 \mathrm{~mm}, \mathrm{~b}$ value $1200 \mathrm{~s} / \mathrm{mm}^{2}, 66$ gradient directions and a single non-diffusion weighted $b=0$ image, acquisition time 7:34 $\min )$.

\section{Adult dataset}

First, for anatomical reference and segmentation, T1-weighted 3D fast gradient echo images were acquired (repetition time $(\mathrm{TR})=8.14 \mathrm{~ms}$, echo time $(\mathrm{TE})=3.73 \mathrm{~ms}$, flip angle $(\mathrm{FA})=8^{\circ}$, cubic voxel size $=1 \mathrm{~mm}$ ). For MWF estimation, whole cerebrum $2 \mathrm{D}$ multi-slice gradient and spin echo (GRASE) images were acquired (TR $=3000 \mathrm{~ms}$, 32 echoes with $10 \mathrm{~ms}$ echo spacing, range 10-320 ms, EPI factor $=3$, Turbo factor $=32$, 26 slices, $1 \mathrm{~mm}$ slice gap, field of view $=$ $240 \times 198 \times 130 \mathrm{~mm}$, acquisition matrix $160 \times 132$, voxel size $=1.5 \times 1.5 \times 4 \mathrm{~mm}$ ) with parallel acquisition (sensitivity encoding, SENSE $=2$ ), and a scan duration of 7:30 minutes. To estimate the reproducibility of tract-specific MWF estimation, a second GRASE scan was acquired for six volunteers [14]. To calculate the tract tracts in the white matter, diffusion weighted images were acquired ( $\mathrm{TR}=7012 \mathrm{~ms}, \mathrm{TE}=74 \mathrm{~ms}$, cubic voxel size $=2 \mathrm{~mm}, \mathrm{~b}$ value $=1200 \mathrm{~s} / \mathrm{mm}^{2}, 66$ gradient directions and a single non-diffusion weighted $\mathrm{b}=0$ image, acquisition time 8:03 $\mathrm{min})$.

\section{Analysis}

\section{Anatomical parcellation}

Using Freesurfer, 68 cortical regions from the Desikan-Killiany atlas as well as 16 subcortical regions were automatically segmented from the T1-weighted images [15]. Subsequently, the T1-weighted image and resulting atlas were resampled to the voxel size of the DTI images (2 $\times 2 \times 2 \mathrm{~mm})$. 


\section{Myelin-water fraction calculation}

To reduce noise in the multi-echo data, a singular value decomposition (SVD) filter [16] was used, as well as a Gaussian filter with a kernel size of $5 \times 5$ and FWHM of $1.1 \mathrm{~mm}$ to spatially smooth the images. Multi-exponential analysis of the multi-echo data was performed using the non-negative least squares (NNLS) algorithm [17]. Using a basis set of 120 logarithmically spaced relaxation functions (T2 range 15 to $2000 \mathrm{~ms}$ ) the NNLS estimates the contribution of each relaxation function to the measured multi-echo data. The algorithm was regularized using an additional minimal energy constraint that allows an increased misfit between 2 and 2.5\% (1.020 $\left.\leq \chi_{\mathrm{reg}} / \chi_{\min } \leq 1.025\right)$ [18]. To model the decay more accurately, both B1 inhomogeneities and imperfect slice profiles were considered, as has been described previously [14]. Subsequently, the MWF was calculated as the ratio of myelin-water associated T2 components (15-40 ms) to all T2 components (15-2000 ms). The MWF maps were co-registered and resliced to the resampled T1-weighted images using the statistical parametric mapping (SPM12, https://www.fil.ion.ucl.ac.uk/spm/software/spm12/) toolkit [19].

\section{Tract tractography}

The preprocessing and tract tracking of the DTI data were performed using ExploreDTI v4.8.6 [20]. The diffusion MRI data was first corrected for head displacement, including Bmatrix rotation, and eddy current induced geometric distortions. Subsequently, an EPI distortion correction was performed by transforming the diffusion images to the resampled T1-weighted space. Whole brain tract tractography was performed using constrained spherical deconvolution with a uniform seed point of $2 \mathrm{~mm}^{3}$, a step size of $1 \mathrm{~mm}$, angle threshold $30^{\circ}$ and tract length range $50-500 \mathrm{~mm}$.

\section{Structural network construction}

The Freesurfer brain regions were used as nodes in the network. Two nodes were considered connected by an edge if there are at least 2 streamlines between them, and if the total tract volume is at least $400 \mathrm{~mm}^{3}$ (= 50 voxels). The latter requirement was added since the myelin maps have thicker slices $(4 \mathrm{~mm})$ compared to the diffusion images $(2 \mathrm{~mm})$, potentially causing unreliable results for tracts with a small volume. Moreover, only the tracts with a fair or better reproducibility of MWF values were included. Therefore, the intra-class correlation coefficient (ICC) was calculated for each tract using a 1-way random model, and only those tracts with (ICC > .40) were selected for further analysis [21]. Furthermore, to avoid results based on a small number of data points, only edges present in at least $50 \%$ of the adults, $50 \%$ of the healthy children and $50 \%$ of the children with CAE were considered.

To construct the MWF weighted network, the median MWF was calculated for each of the tract tracts connecting nodes. The median, instead of the mean, was used, since the MWF distribution in a region of interest is skewed [14], [22], [23]. 


\section{Maturation analysis}

The effect of age on the tract-specific myelin content was derived from the healthy subjects using linear regression. Previous studies suggest a non-linear, reversed U-shaped, relation between myelin content and age [9], therefore a quadratic age term was added to the model.

Using the regression formula, the tract-specific myelin-water content was calculated for the representative ages of $12\left(\mathrm{MWF}_{12 \mathrm{y}}\right)$ and $25\left(\mathrm{MWF}_{25 \mathrm{y}}\right)$ years. Subsequently, the percentual difference in myelin-water content (PDMW) at 25 years compared to 12 years was calculated as;

$$
P D M W=\frac{M W F_{25 y}-M W F_{12 y}}{M W F_{12 y}} * 100 \%
$$

Note that the PDMW can also yield negative values, indicating a decrease in myelinwater content.

A tract-specific myelin developmental atlas was obtained by calculating the PDMW for each tract. The atlas can be used to differentiate tracts based on their myelin development.

To investigate developmental differences between healthy children and children with CAE, two types of tracts were considered. The tracts that show the least myelin increase (the $10 \%$ tracts with the lowest PDMW) and the tracts with a high increase of myelin content (the $10 \%$ tracts with the highest PDMW).

\section{Statistical analysis}

Differences in PDMW values in the healthy controls were examined between the tracts only traversing inside the left and right hemispheres (i.e. intra-hemispheric tracts) using an independent samples t-test. Using a similar approach, differences between intra- and interhemispheric, anterior (frontal lobe) and posterior (occipital and parietal lobes) as well as central (sub-cortical, cingulate and insular regions) and peripheral connections were assessed.

Differences in myelin-water content of early and late developing tracts between the healthy children and children with CAE were estimated using multivariate regression analysis. Age and sex were added to the model as covariates. To evaluate the robustness of the results, the analysis was repeated for $25 \%$ and $50 \%$ of the tracts (instead of $10 \%$ ) with the lowest/highest PDMW values.

Statistical significance was inferred when $p<.05$. 


\section{Results}

In Figure 6.1 the mean MWF of all the tracts is shown as a function of age. The solid curve represents a least squares quadratic fit $\left(R^{2}=.77, p<.01\right)$.

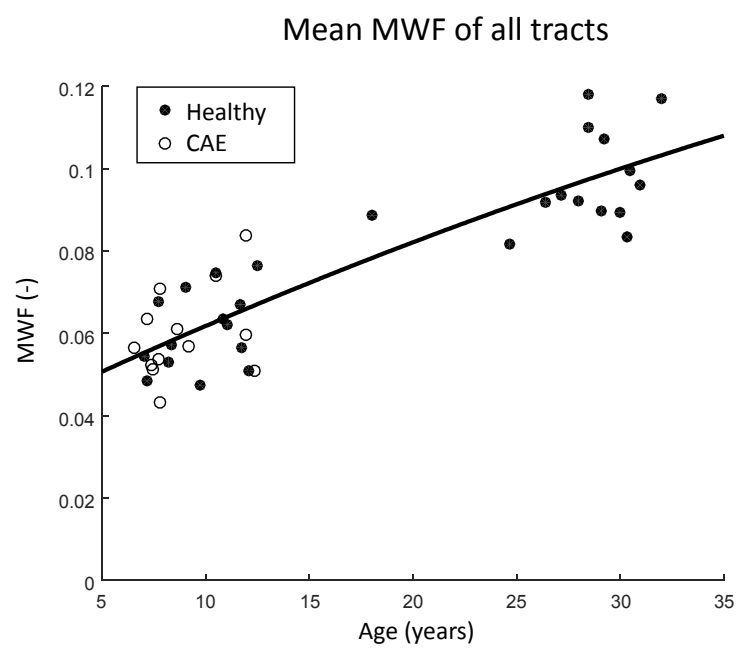

Figure 6.1: Mean MWF of all tracts of healthy subjects and children with CAE with respect to age. The solid curve represents a quadratic least-squares fit.

The tract-specific PDMW atlas from childhood (12y) to adulthood (25y) is shown in Figure 6.2A, a histogram with the PDMW values is shown in Figure 6.2B. Figure 6.2C shows the $10 \%$ tracts with the lowest MWF increase, and Figure 6.2D the $10 \%$ tracts with the highest MWF increase. Table 6.2 shows the PDMW for several groups of tracts. The PDMW values of the tracts in the right hemisphere, intra-hemispheric tracts, and peripheral tracts are significantly higher compared to tracts in the left hemisphere, inter-hemispheric tracts, and central tracts, respectively. PDMW values of anterior tracts did not differ significantly from posterior tracts.

No significant differences were found in MWF content between healthy children and children with CAE for either the tracts that develop in an early stage $(\beta=-.15, p=.49)$ (Figure 6.2C), or the tracts that develop in a later stage $(\beta=.26, p=.20)$ (Figure 6.2D). However, the difference between the MWF of late and early developing tracts is significantly smaller in the children with CAE $(\beta=-.58, p<.01)$ (Figure 6.3). Note that the selection of early and late developing tracts was based on healthy controls only.

When selecting $25 \%$ or $50 \%$ of the tracts (instead of $10 \%$ ) with the highest and lowest PDMW values the results are similar, showing that the difference between the MWF of late and early developing tracts $(\Delta)$ is significantly smaller in the children with CAE $(p<.01)$. 
Table 6.2: PDMW values for several tract types in healthy controls.

\begin{tabular}{lll}
\hline Tract types & PDMW $($ mean \pm SD) & $p$-value \\
\hline Left hemisphere & $40 \pm 13 \%$ & $<.01$ \\
Right hemisphere & $45 \pm 16 \%$ & \\
\hline Intra-hemisphere & $42 \pm 14 \%$ & $<.01$ \\
Inter-hemisphere & $35 \pm 10 \%$ & .13 \\
\hline Anterior & $34 \pm 9 \%$ & \\
Posterior & $36 \pm 12 \%$ & $<.01$ \\
\hline Central & $19 \pm 17 \%$ & \\
Peripheral & $43 \pm 14 \%$ & \\
\hline
\end{tabular}

Abbreviations: PDMW, percentual differences in myelin-water content; SD, standard deviation.

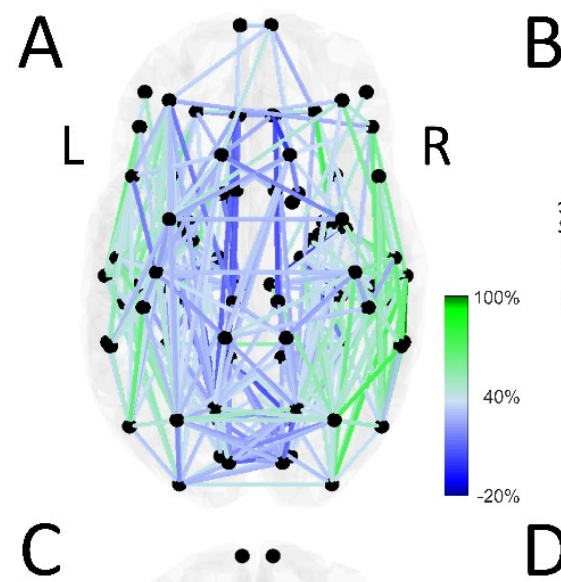

B
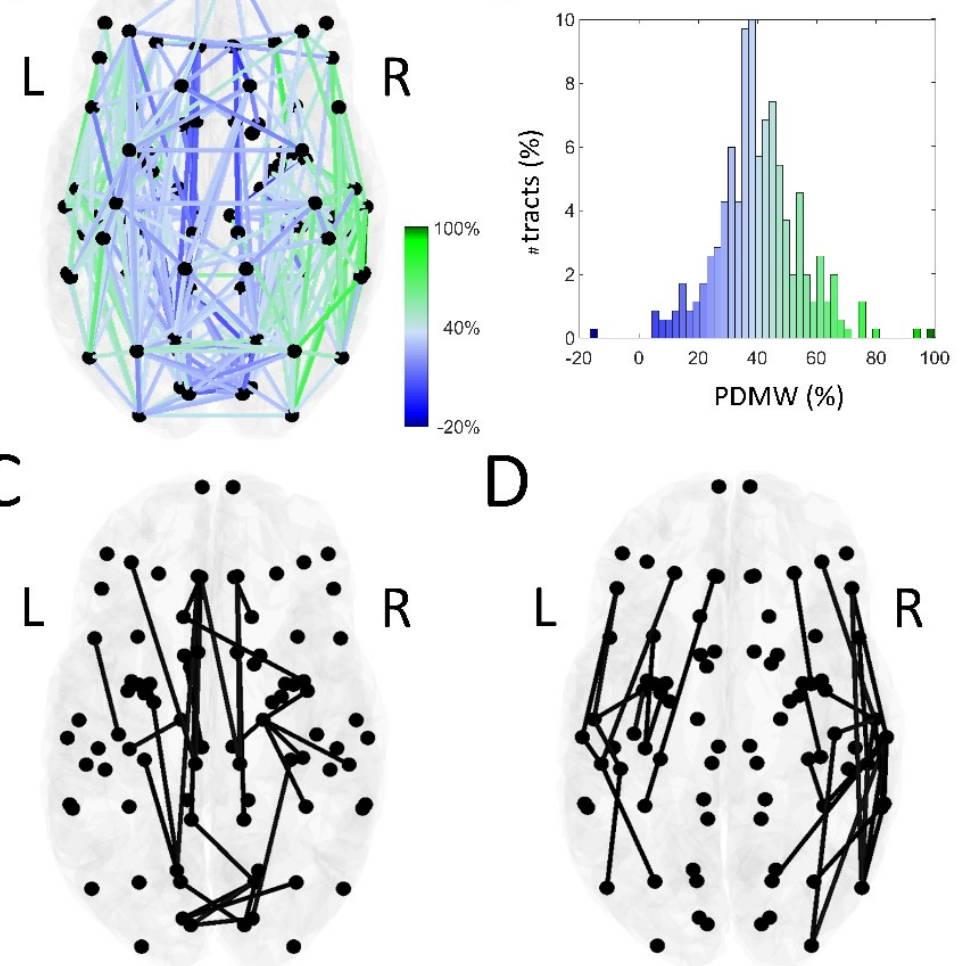

D

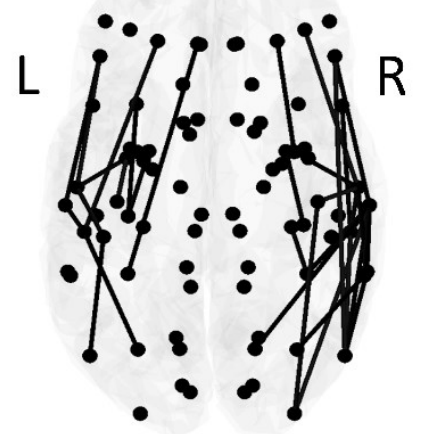

Figure 6.2: A) Myelin development atlas, showing the tract-specific PDMW values, B) histogram of the PDMW values, C) the $10 \%$ tracts with the lowest percentual MWF increase, and D) the $10 \%$ with the highest percentual MWF increase. 


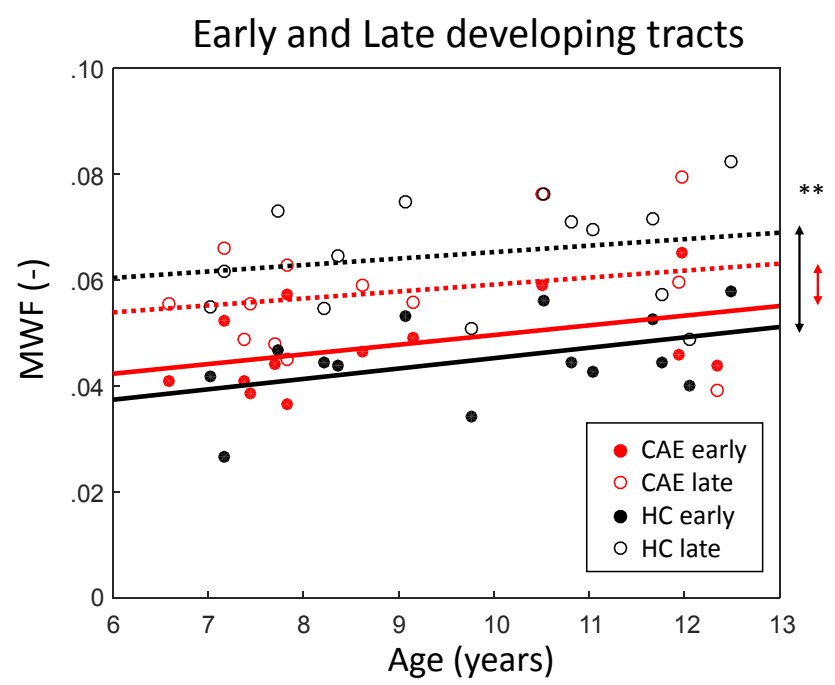

Figure 6.3: Myelin-water content of early (solid lines and closed circles) and late developing tracts (dashed lines and open circles) in children with childhood absence epilepsy (CAE; red) and healthy peers (HC; black). The difference between the myelin-water fraction (MWF) of late and early developing tracts is significantly smaller in children with CAE (red arrow) compared to controls (black arrow). ${ }^{* *}, \mathrm{p}<.01$.

\section{Discussion}

\section{Current findings}

This study characterized a whole brain tract-specific myelin development atlas for children aged from 7 to 12 years and adults aged from 18 to 32 years, and assessed differences of tract-specific myelin-water content in children with CAE. Tract-specific myelin-water content is shown to develop through adulthood. Especially, the tracts connecting central regions, inter-hemispheric connections and tracts in the left hemisphere are shown to be myelinated in an earlier stage compared to the peripheral regions, intra-hemisphere and right hemisphere connections, respectively. Furthermore, cross-sectional differences in myelin-water content of early and late developing tracts (as determined in healthy controls) were found between children with CAE and healthy controls.

\section{Maturation of myelin content}

Myelination proceeds rapidly after birth and during infancy and early childhood [3], [24]. Here, the myelin content is shown to increase from childhood through adolescence into 
adulthood, as was also reported previously [25], [26]. By determining the development of tract-specific myelin content, different development trajectories can be observed for different tract bundles. Early myelination is considered to develop from inward to outward regions and from posterior to anterior regions [27], which is partly in agreement to our current findings. While the tracts connecting central structures (sub-cortical, cingulate and insular) are developed in an earlier stage compared to outward regions, no difference was found between tracts in posterior and anterior regions. This possibly indicates that the posterior-anterior development of myelin content precedes largely the age of $12 \mathrm{y}$, while the inward-outward pattern persists into adulthood.

The tracts in the left hemisphere had lower PDMW values compared to the right hemisphere, indicating that the tracts in the left hemisphere are myelinated in an earlier stage. In most individuals, language processes are strongly left-lateralized [28], [29]. Similar leftward asymmetries in myelin-water content were previously related to language ability in young children (aged 1-5 years) [30]. Although language development peaks at these early ages, it continues through adolescence into adulthood [31]. Therefore, the persisting asymmetry that we observed between 12 and 25 years of age is likely to be related to language development.

\section{Myelin abnormalities in CAE}

Children with CAE showed cross-sectional differences in myelin-water content of tracts with different maturation trajectories, as determined in healthy controls. Namely, children with CAE showed a lower difference in myelin-water content between the earlier and later developing tracts. This finding may exhibit the effect that epilepsy has on the development of various white matter tracts during childhood. The widespread myelin developmental differences might play a role in the underlying mechanisms of CAE. The main function of myelin is accelerating the propagation velocity of electrical signals via saltatory conduction. Differences in myelin content could impact the complex temporal organization of action potentials [32] and therefore might play a role in the synchronized neuronal activity during generalized seizures that accompany CAE. A previous study with the same cohort already showed a decreased myelin-water content in the frontal lobe of children with CAE [6]. Thus, besides a lobular decrease of frontal myelin content shown earlier, this study shows a more widespread difference in neurodevelopment as part of CAE.

\section{Study considerations}

Our study has important strengths. The study used well defined inclusion criteria for children with CAE in agreement with current ILAE standards. Moreover, the age and sexmatched controls enabled us to make reliable group-level comparisons. One major limitation of the study is the different acquisition resolution of the diffusion and GRASE 
images, resulting in additional preprocessing steps (interpolation, co-registration) that could introduce artifacts in the results. Accordingly, only tracts of sufficient volume (50 voxels) and reproducible MWF values were included in the analysis. Additionally, diffusion MRI acquisition is prone to EPI distortions, especially the frontal and temporal lobe are susceptible to such artifacts. While an EPI correction was performed, it cannot be excluded that some regions are still affected by EPI distortions. Furthermore, healthy development of myelin-content between the ages of 12 and 25 was assessed cross-sectionally. While our results indicate that the patterns of maturation presented are in agreement with most evidence from other studies, a more accurate overview of maturation effects should be made with longitudinal data. Similarly, the neurodevelopmental difference in CAE is based on the maturation atlas from healthy controls. Moreover, the sample size of this study is relatively small, which limits the power for more in-depth correlation analysis with clinical/epilepsy variables. Future larger studies with longitudinal data, or even adults who were previously diagnosed with $\mathrm{CAE}$, could provide additional insights into the neurodevelopmental involvement of myelin content in CAE.

\section{Conclusion}

This study investigated the development of healthy tract-specific myelin-water content. Myelin content was found to increase steadily throughout the whole brain from childhood into adulthood. Clear patterns of maturation were observed, where tracts in the central regions myelinate prior to peripheral tracts and intra-hemispheric tracts as well as tracts in the left hemisphere myelinate prior to inter-hemispheric tracts and tracts in the right hemisphere, respectively. The latter possibly due to language development. The development atlas was applied to a cohort of children with CAE and healthy controls. The difference between earlier and later myelinated tracts was lower in children with CAE, indicating that CAE is associated with widespread neurodevelopmental myelin differences. 


\section{References}

[1] M. Baraban, S. Mensch, and D. A. Lyons, "Adaptive myelination from fish to man," Brain Res., vol. 1641, pp. 149-161, 2016.

[2] J. Valk and M. S. van der Knaap, "Myelination and Retarded Myelination," in Magnetic Resonance of Myelin, Myelination, and Myelin Disorders, Berlin, Heidelberg: Springer Berlin Heidelberg, 1989, pp. 26-65.

[3] D. P. Carmody, S. M. Dunn, and A. S. Boddie-willis, "A quantitative measure of myelination development in infants, using MR images," Neuroradiology, vol. 46, pp. 781-786, 2004.

[4] E. Kavroulakis et al., "Myelin content changes in probable Alzheimer's disease and mild cognitive impairment: Associations with age and severity of neuropsychiatric impairment," $J$. Magn. Reson. Imaging, vol. 47, no. 5, pp. 1359-1372, 2018.

[5] S. Kolind et al., "Clinical Brain and cord myelin water imaging: a progressive multiple sclerosis biomarker," NeuroImage Clin., vol. 9, pp. 574-580, 2015.

[6] G. S. Drenthen et al., "Lower myelin-water content of the frontal lobe in childhood absence epilepsy," Epilepsia, vol. 60, no. 8, pp. 1689-1696, 2019.

[7] D. C. Dean III et al., "Alterations of Myelin Content in Parkinson's Disease : A Cross-Sectional Neuroimaging Study," PLoS One, vol. 11, no. 10, p. e0163774, 2016.

[8] D. J. M. Lang et al., "Clinical 48 echo T2 myelin imaging of white matter in first-episode schizophrenia: Evidence for aberrant myelination," NeuroImage Clin., vol. 6, pp. 408-414, 2014.

[9] C. Lebel, S. Treit, and C. Beaulieu, "A review of diffusion MRI of typical white matter development from early childhood to young adulthood," NMR Biomed., vol. 32, no. 4, p. e3778, 2019.

[10] C. Laule et al., "Magnetic resonance imaging of myelin," Neurotherapeutics, vol. 4, no. 3, pp. 460-484, Jul. 2007.

[11] E. Alonso-Ortiz, I. R. Levesque, and G. B. Pike, “MRI-based myelin water imaging: A technical review," Magn. Reson. Med., vol. 73, no. 1, pp. 70-81, Jan. 2015.

[12] T. R. Baumeister, S. H. Kolind, A. L. MacKay, and M. J. McKeown, "Inherent spatial structure in myelin water fraction maps," Magn. Reson. Imaging, Oct. 2019.

[13] M. Mancini et al., "Introducing axonal myelination in connectomics: A preliminary analysis of g-ratio distribution in healthy subjects," Neuroimage, vol. 182, no. June 2017, pp. 351-359, 2017.

[14] G. S. Drenthen, W. H. Backes, A. P. Aldenkamp, and J. F. A. Jansen, "Applicability and reproducibility of $2 \mathrm{D}$ multi-slice GRASE myelin water fraction with varying acquisition acceleration," Neuroimage, vol. 195, pp. 333-339, 2019.

[15] R. S. Desikan et al., "An automated labeling system for subdividing the human cerebral cortex on MRI scans into gyral based regions of interest," Neuroimage, vol. 31, pp. 968-980, Jul. 2006.

[16] M. Bydder and J. Du, "Noise reduction in multiple-echo data sets using singular value decomposition," Magn. Reson. Imaging, vol. 24, no. 7, pp. 849-856, Sep. 2006.

[17] K. P. Whittall and A. L. MacKay, "Quantitative interpretation of NMR relaxation data," J. Magn. Reson., vol. 84, no. 1, pp. 134-152, Aug. 1989.

[18] M. G. Skinner, S. H. Kolind, and A. L. MacKay, "The effect of varying echo spacing within a multiecho acquisition: better characterization of long T2 components," Magn. Reson. Imaging, vol. 25, no. 6, pp. 840-847, Jul. 2007.

[19] K. J. Friston, J. Ashburner, S. Kiebel, T. Nichols, and W. Penny, Eds., Statistical Parametric Mapping. Elsevier, 2007.

[20] A. Leemans, B. Jeurissen, J. Sijbers, and D. Jones, “ExploreDTI: a graphical toolbox for processing, analyzing, and visualizing diffusion MR data," Proc. 17th Sci. Meet. Int. Soc. Magn. Reson. Med., vol. 17, no. 2, p. 3537, 2009.

[21] D. V. Cicchetti, "Guidelines, criteria, and rules of thumb for evaluating normed and standardized assessment instruments in psychology.," Psychol. Assess., vol. 6, no. 4, pp. 284290, 1994. 
[22] S. Kolind, L. Matthews, H. Johansen-berg, M. I. Leite, and C. R. Steven, "Myelin Water Imaging Reflects Clinical Variability in Multiple Sclerosis," Neuroimage, vol. 60, pp. 263-270, 2012.

[23] M. Dayan et al., "MRI Analysis of White Matter Myelin Water Content in Multiple Sclerosis : A Novel Approach Applied to Finding Correlates of Cortical Thinning," Front. Neurosci., vol. 11, p. 284, 2017.

[24] A. L. Mackay and C. Laule, "Magnetic Resonance of Myelin Water: An in vivo Marker for Myelin," vol. 2, pp. 71-91, 2016.

[25] M. Arshad, J. A. Stanley, and N. Raz, "Adult age differences in subcortical myelin content are consistent with protracted myelination and unrelated to diffusion tensor imaging indices," Neuroimage, vol. 143, pp. 26-39, Dec. 2016.

[26] B. L. Geeraert, R. M. Lebel, and C. Lebel, "A multiparametric analysis of white matter maturation during late childhood and adolescence," Hum. Brain Mapp., vol. 40, no. 15, pp. 4345-4356, 2019.

[27] R. Dietrich et al., "MR Evaluation of Early Myelination Patterns in Normal and Developmentally Delayed Infants than white," Am. J. Roentgenol., vol. 150, no. 4, pp. 889-896, 1988.

[28] S. Ocklenburg et al., "Neurite architecture of the planum temporale predicts neurophysiological processing of auditory speech," no. July, pp. 1-9, 2018.

[29] K. Hugdahl, "Lateralization of cognitive processes in the brain," vol. 105, pp. 211-235, 2000.

[30] J. O. Muircheartaigh et al., "Interactions between White Matter Asymmetry and Language during Neurodevelopment," vol. 33, no. 41, pp. 16170-16177, 2013.

[31] M. A. Nippold, "Language Development during the Adolescent Years : Aspects of Pragmatics , Syntax , and Semantics," Top. Lang. Disord., vol. 20, no. February, pp. 15-28, 2000.

[32] S. Pajevic, P. J. Basser, and R. D. Fields, "Role of myelin plasticity in oscillations and synchrony of neuronal activity," Neuroscience, vol. 276, pp. 135-147, 2014. 



\section{Chapter 7}

\section{Functional brain integrity is related to epilepsy severity in childhood absence epilepsy}

Gerhard S. Drenthen*, Floor Fasen*, Eric L.A. Fonseca Wald, Walter H. Backes, Albert P. Aldenkamp, R. Jeroen Vermeulen, Mariette H.J.A. Debeijvan Hall, Jos G.M. Hendriksen, Sylvia Klinkenberg, Jacobus F.A. Jansen

*Authors contributed equally

Submitted 


\section{Abstract}

Childhood absence epilepsy (CAE) is generally considered to be refrained from cognitive impairments, although some recent studies report cognitive, mainly attentional, deficits. Here we set out to investigate the whole brain functional network of children with CAE and controls. Furthermore, the possible relation of the functional network abnormalities with epilepsy and neurocognitive characteristics is studied.

Seventeen children with childhood absence epilepsy (aged $9.2 \pm 2.1$ years) and 15 age and sex-matched controls (aged $9.8 \pm 1.8$ years) were included. Resting state functional MRI was acquired to study the functional network. Using graph theoretical analysis, three global metrics of the functional network were investigated: the characteristic path length, the clustering coefficient, and the small-worldness. A multivariable linear regression model including age, sex, and subject motion as covariates was used to investigate group differences in the graph metrics. Subsequently, relations of the graph metrics with epilepsy and neurocognitive characteristics were assessed.

Longer path lengths, weaker clustering and a lower small-world network topology were observed in children with CAE compared to controls. Moreover, longer path lengths were related to a longer duration of CAE and a higher seizure frequency. Clustering and smallworldness were not significantly related to epilepsy or neurocognitive characteristics.

The organization of the functional network of children with CAE is less efficient compared to controls, and is related to disease duration, and is therefore suggested to be a part of the underlying mechanism of the pathology. 


\section{Introduction}

Childhood absence epilepsy (CAE) constitutes approximately $12 \%$ of all types of childhood epilepsies [1]. Children with CAE suffer from frequent absence seizures that occur multiple times per day [2], [3], often in clusters. Absence seizures typically last around 10 seconds, during which the child is unresponsive to the environment [4]. At these times, generalized 3 $\mathrm{Hz}$ spike-and-wave discharges (GSWD) can be observed in an electroencephalogram (EEG) [3], [5]. The onset of CAE is usually between the ages of 5 and 10 years [2], [6]. Most children with CAE will outgrow the seizures when reaching adulthood, therefore CAE has traditionally been considered a benign syndrome [7]. However, recent reports indicate a broad spectrum of abnormal behavioural and cognitive performance in CAE, including deficits in attention, memory, processing speeds, and language [8], [9]. Furthermore, these deficits in cognitive performance may persist, even after becoming seizure-free [10].

Previous research has shown that children with CAE have altered resting-state functionality in selected networks of the brain [3], [11], [12]. Differences in functional connectivity have been reported using independent component analysis (ICA), particularly in dorsal attention, salience, and default mode networks [3]. Furthermore, disruptions of hubs in the functional network were found for the default mode network and the thalamus [12]. Moreover, a less efficiently organized whole brain structural network in children with CAE was previously reported using diffusion weighted imaging [13]. Besides these microstructural changes, based on the clinical profile and cognitive comorbidities of this allegedly benign syndrome [14], we would also expect whole brain functional disruptions. While the whole brain functional network has already been shown to be reorganized during absence seizures [15], currently the involvement of the resting state functional network in CAE (i.e. interictally) on a whole brain level remains undetermined. Whole brain network metrics can be calculated using graph theoretical analysis. Graph theory was developed as a way of representing pairwise relations between objects, using vertices (or nodes) and their relations defined as edges (or connections) [16]. For the brain, the nodes represent brain regions and the edges are the connections between the brain regions [17]. In case of functional magnetic resonance imaging (fMRI), these connections do not describe the physical structural connections, but rather their temporal correlations (e.g. functional connectivity).

In this study, we will investigate characteristics of functional connectivity in children with CAE and controls by means of graph theoretical analysis. Two global graph metrics, the characteristic path length and the clustering coefficient, will be used to quantify the functional brain network in terms of integration and segregation, respectively [16], [18]. Moreover, the overall topology of the networks will be assessed using the small-worldness, which is indicative of the overall organization of the networks [19]. Subsequently, we will assess whether the global graph metrics differ between children with CAE and controls. Thereafter, we aim to explore the potential relation of the global graph metrics with epilepsy characteristics and neurocognitive performance. 


\section{Methods}

\section{Participants}

Seventeen children with a clinical diagnosis of CAE (aged 6-12y) were included, the inclusion criteria were reported previously [20]. Additionally, fifteen age-sex matched controls (aged 7$12 \mathrm{y}$ ) were included for comparison. All caregivers, and participants aged $\geq 12$ years old gave written permission prior to inclusion in the study and this research was approved by the medical ethics committee azM/UM NL55455.068.15/METC152055 and is listed at clinicaltrials.gov under NCT02954107. The following epilepsy characteristics were recorded: age at onset, duration of epilepsy and number of GSWD per hour. The latter was determined using a 24h-EEG, where the number of GSWD that lasted at least 3 seconds were counted in three separate and randomly chosen hours during which the patient was in a wakeful state. The mean of the three measurements was used as a measure of seizure frequency. Five children with CAE were already successfully treated with anti-epileptic drugs (AEDs), and were therefore omitted from any subsequent analysis with seizure frequency.

For all participants, general intelligence and processing speed was determined using six subtests (mean = 10; SD = 3) of the Dutch version of the Wechsler Intelligence Scale for Children third edition (WISC-III) (similarities, vocabulary, picture completion, coding, block design, and symbol search). The subject characteristics are shown in Table 7.1. The two groups did not differ significantly regarding age, sex, and general intelligence. Processing speed index (mean =100; $\mathrm{SD}=15)$ was significantly lower for children with CAE compared to controls (95 \pm 15 vs. $108 \pm 14, p=.03)$.

\section{MRI acquisition}

All subjects were scanned on a 3.0 T unit (Philips Achieva, Best, the Netherlands) using a 32element phased array coil. First, for anatomical reference and segmentation, T1-weighted (T1w) 3D turbo field echo images were acquired (repetition time (TR) $=8.36 \mathrm{~ms}$, echo time $(\mathrm{TE})=3.84 \mathrm{~ms}$, flip angle $(\mathrm{FA})=8^{\circ}$, voxel size $\left.=1 \times 1 \times 1 \mathrm{~mm}\right)$. Functional MR images were acquired in resting state with eyes closed using a single-shot echo planar imaging (EPI) sequence $(\mathrm{TR}=2000 \mathrm{~ms}, 31$ slices, $\mathrm{TE}=35 \mathrm{~ms}$, pixel size $=2 \times 2 \mathrm{~mm}, 4 \mathrm{~mm}$ thick slices, and 195 acquisitions). 
Table 7.1. Subject characteristics of children with CAE and controls.

\begin{tabular}{llll}
\hline & CAE & Controls & p-value \\
\hline Number $(\#)$ & 17 & 15 & - \\
Age $(\mathrm{y}$, mean $\pm \mathrm{SD})$ & $9.2 \pm 2.1$ & $9.8 \pm 1.8$ & .37 \\
Sex $(\mathrm{M} / \mathrm{F})$ & $12 / 5$ & $11 / 4$ & .86 \\
Handedness (R/L) & $16 / 1$ & $11 / 4$ & .11 \\
Age of onset (y, mean $\pm \mathrm{SD})$ & $8.0 \pm 2.0$ & - & - \\
Duration of epilepsy (y, mean \pm SD) & $1.2 \pm .74$ & - & - \\
normalized duration of epilepsy (mean $\pm \mathrm{SD})$ & $.15 \pm .35$ & - & - \\
Average GSWD per hour (mean \pm SD) & $6.3 \pm 7.0$ & - & - \\
Schooling (regular/special) & $16 / 1$ & $15 / 0$ & .34 \\
Family history of epilepsy (Yes/No) & $2 / 15$ & $1 / 14$ & .62 \\
WISC-III Subtests (mean \pm SD) & $9.6 \pm 1.9$ & $10.9 \pm 2.3$ & .09 \\
WISC-III Processing speed index (mean \pm SD) & $95 \pm 15$ & $108 \pm 14$ & .03 \\
\hline
\end{tabular}

Abbreviations: Y, years; SD, standard deviation; L, left; R, right; $C A E$, childhood absence epilepsy; WISC-III, Wechsler Intelligence Scale for Children, third edition; GSWD, generalized spike wave discharges.

\section{Analysis}

\section{Preprocessing}

Preprocessing of the fMRI data and T1w structural images was performed using the Statistical Parametric Mapping software package, SPM12 (https://www.fil.ion.ucl.ac.uk/spm/) in Matlab R2016b. The structural images were automatically parcellated into 68 cortical and 14 subcortical regions using Freesurfer (version 5.1) based on the Desikan-Killiany atlas [21], [22].

First, a slice timing correction was applied to the functional images. Second, to correct for head displacement, all slices were computationally realigned to the first volume in the sequence. Third, the functional images were smoothed through convolution with a $6 \mathrm{~mm}$ fullwidth half maximum (FWHM) Gaussian kernel. Fourth, a band-pass filter of 0.01 to $0.1 \mathrm{~Hz}$ was applied to extract the frequency band of interest. Fifth, the first 5 volumes of the functional images were discarded to ensure steady-state longitudinal magnetization of the blood-oxygen level dependent (BOLD) signal [3], [23]. Last, the time signals of each region of interest were averaged and corrected for head motion (relative translational movement [24]) as well as white matter and cerebrospinal fluid (CSF) signals via linear regression in order to reduce the contribution of physiological noise [16]. To cope with EPI distortions in the fMRI data, the T1w image and corresponding Freesurfer atlas were non-linearly co-registered to the functional images using Elastix v4.9.0 [25]. Subsequently, a mask based on the outline of the brain in the fMRI data was used to prevent that fMRI voxels outside brain regions were included. 
Since we included young children in our study, subject motion is likely to affect our results. Therefore, mean relative motion parameters for translational and rotational movement were included in the statistical analysis as covariates [24].

\section{Functional network construction}

For each pair of regions, the connection strength was calculated using the Pearson's correlation coefficients, resulting in a weighted adjacency matrix representing the functional network. Negative correlations were set to zero because currently the role of negative weights in the functional network is unclear [18]. On average, only $2 \%$ of the correlations were negative. Additionally, to cope with noise and false positive connections, non-significant correlation coefficients in the connectivity matrix were set to zero.

\section{Network analysis}

The functional networks are quantitatively described by two of the most robust and widely applied global graph metrics, the characteristic path length $(L)$ and clustering coefficient $(C)$ [19]. $L$ provides insight into how well information can spread throughout a network, while $C$ is a measure of local information processing. A network with strongly clustered modules and relative short path length between nodes is considered an efficiently organized small-world network. Graph analysis is highly dependent on the topology of the network [26]. To obtain global graph metrics that are normalized with respect to variations in topology, the global graph metrics are determined relative to the average of 100 random networks with similar degree distribution $\left(\lambda=L / L_{\text {rand }}\right.$ and $\left.\gamma=C / C_{\text {rand }}\right)$. A measure of small-worldness can now be defined as $\sigma=\gamma / \lambda$, where $\sigma>1$ indicates that a network has a small-world topology. Furthermore, only networks with the same number of nodes and edges (i.e. the networks are equally sparse) will be compared. Moreover, to decrease the occurrence of false positives and false negatives in the network, only the nodes and edges present in the network of at least half of the subjects are considered in the graph analysis (i.e. group thresholding) [27]. The number of edges in each network is varied such that the functional networks are $60-90 \%$ sparse, with intervals of $1 \%$. Networks sparser than $90 \%$ would lead to disconnected nodes, hindering the graph analysis. Graph metrics were calculated using the Brain Connectivity Toolbox (http://www.brain-connectivity-toolbox.net).

\section{Statistical analysis}

Between-group differences in the global graph metrics were assessed using multivariable linear regression models. To account for demographical differences and head motion, age, sex and the two mean motion parameters were added to the models as covariates. Prior to the analysis, the non-normally distributed mean motion parameters and number of GSWD per hour were transformed using the Box-Cox transformation [28]. Furthermore, the motion parameters are inherently correlated $(\mathrm{r}=.88, \mathrm{p}<.01)$. Therefore, to prevent multicollinearity in the regression model, only the mean translation motion parameter was added to the model. 
To assess whether the graph metrics relate to epilepsy and cognitive characteristics, linear multivariable regression models were used, correcting for the effects of age, sex and head motion. For the children with $\mathrm{CAE}$, the relation of the global graph metrics with the duration of CAE, number of GSWD per hour, general intelligence, and processing speed index was assessed using linear regression models, correcting for the effects of age, sex and head motion. Furthermore, in the control group, the relation of global graph metrics were with the general intelligence and processing speed index was assessed using linear regression models, correcting for the effects of age, sex, and head motion.

Statistical significance was inferred when $\mathrm{p}<.05$.

\section{Results}

\section{Between-group differences}

The graph metrics of children with CAE and controls are plotted with respect to the sparsity level in Figure 7.1. The normalized characteristic path length was significantly higher in children with CAE compared to controls for networks that are $75 \%$ and $78-83 \%$ sparse. The normalized clustering coefficient was found to be lower in children with CAE compared to controls, reaching statistical significance over a wide sparsity range (67-90\%). Similarly, the small-worldness was significantly lower in children with CAE compared to controls, over nearly the whole sparsity range (62-90\%).
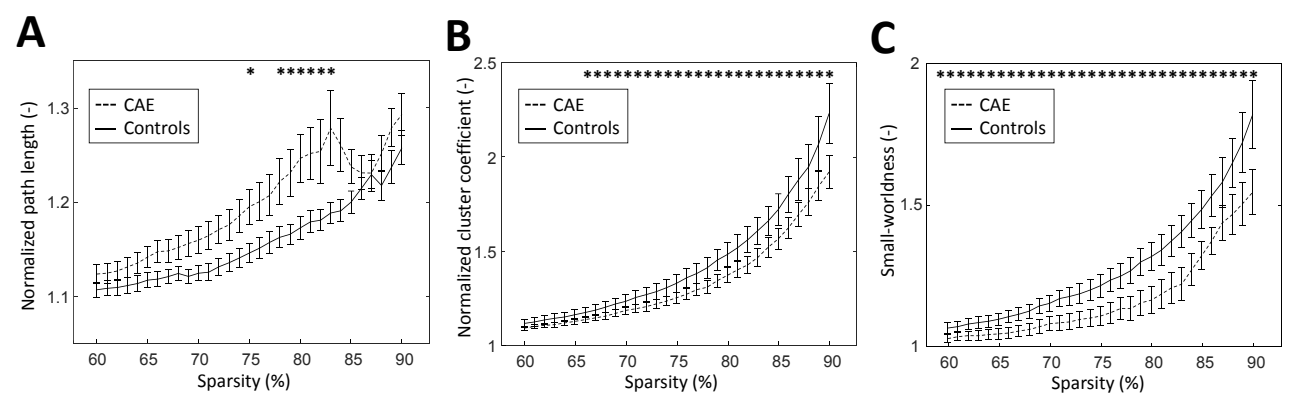

Figure 7.1: A) Normalized path length, B) normalized cluster coefficient and C) normalized smallworldness as a function of sparsity. Mean and standard errors for children with CAE (dashed) and controls (solid) are shown. Asterisks indicate a significant difference between the two groups $(p<.05)$. Note: for the purpose of visualization, the graph metrics corrected for the effects of age, sex, and motion are depicted. 


\section{Epilepsy and neurocognitive characteristics}

The normalized path length was found to be positively related to the duration of CAE, reaching significance for sparsity values $82 \%$ and $83 \%$. Furthermore, the normalized path length related significantly to the seizure frequency for sparsity values $68 \%$, and $70-81 \%$. The normalized clustering coefficient and small-worldness did not significantly relate to either the normalized duration of CAE nor seizure frequency $(p>.10)$. Figure 7.2 shows the normalized path length of networks for a representative sparsity of $82 \%$ as a function of the duration of CAE (A) and as a function of the GSWD per hour (B).

The graph metrics did not relate significantly to general intelligence or processing speed index in either the control or the CAE group.
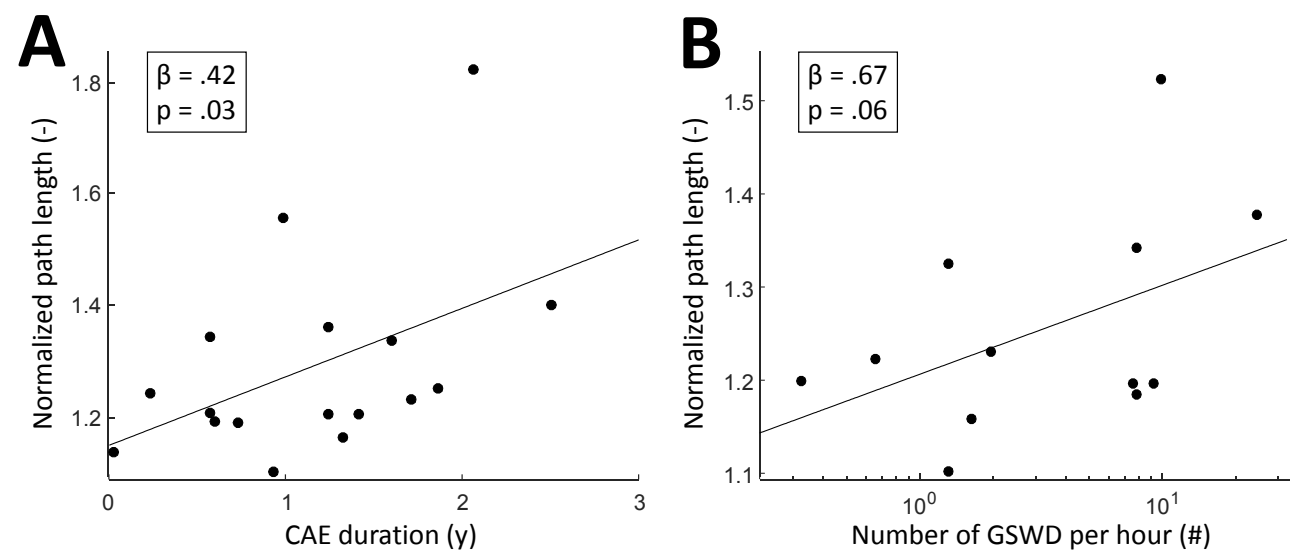

Figure 7.2: The normalized path length of networks for a representative sparsity of $82 \%$ as a function of the duration of CAE (A) and as a function of the GSWD per hour (B).

\section{Discussion}

\section{Current findings}

In the current study, we aimed to study alterations in the global functional network of children with CAE compared to controls on a whole brain level using graph theoretical analysis. We have shown that the functional network organization is impaired in children with CAE, revealing a less efficiently organized network in terms of a weaker clustering and less small-world organized networks. Furthermore, a longer duration of CAE and higher seizure frequency is related to longer path lengths (i.e. more deviant compared to controls), illustrating a relationship with disease development. Clustering and small-worldness were 
not significantly related to epilepsy characteristics. None of the graph metrics were related to general intelligence in either the controls or children with CAE.

\section{Between-group differences}

Weaker clustering and lower small-worldness are reported in children with CAE compared to controls, showing that the network topology exhibits a less small-world organization. These results indicate that the functional brain network of children with CAE is organized in a less efficient manner compared to controls. Previously, similar findings of weaker clusters and lower small-worldness were reported in the structural network of children with CAE using diffusion weighted imaging [13]. Combined with the results from our study, this implies a disruption of the brain network on a functional as well as structural level. To date, reports on functional network changes in CAE have mainly focussed on the default mode network and the attention networks (dorsal and salience) [3], [5], [11], [12], [29]. Possibly, our results could be related to some extent to the underlying impairments of either the default mode or attention networks. However, here we showed that the functional alterations are not limited to certain resting state networks, but rather extend to the functional brain network as a whole. This provides additional insights that not focus on particular (e.g. attentional) deficits in CAE, but rather on the broad spectrum of behavioural, cognitive and linguistic comorbidities of CAE [8]. Previously, it was shown that the whole brain functional network reorganized during and after a GSWD [15]. The results of this study extend upon this by showing that the inter-ictal functional brain network also seems to be affected.

\section{Relationship of network metrics with epilepsy characteristics}

Longer path lengths were found to be associated with a longer duration of CAE and more GSWD per hour. Networks with high degrees of clustering and short path lengths are considered efficient networks with a small-world organization [19]. Therefore, longer paths indicate a less optimal network organization and could be a contributing factor to the pathology. Previously using fMRI, the local functional network disruptions in CAE were also related to the duration of CAE. More specifically, a longer duration of CAE was related to a lower degree centrality in the precuneus [12], left-lateralization of functional connectivity in the anterior insula [11] and decreased functional connectivity in the superior frontal gyrus and lateral parietal cortex [5]. While these studies vary in methodology, they share that more aberrant network properties from the 'normal situation' (i.e. controls) is related to longer duration of CAE. Similarly, in the current study the children with CAE showed longer path lengths compared to controls, indicating that suffering from CAE for a longer time is associated with a more abnormal state of the functional network. Furthermore, a less efficiently and more randomly integrated network was previously associated with poor seizure control in temporal lobe epilepsy (TLE) patients [30]. Although the clinical profile of 
TLE differs from our study, this further indicates that an altered integration might be related to a worse seizure control.

\section{Neurocognitive performance}

A lower neurocognitive performance for the processing speed index and a trend towards lower general intelligence was observed in children with CAE. This is in agreement with previous studies on neurocognition in CAE [9], [31]. Furthermore, more efficient global functional networks in terms of clustering and higher degrees of small-world topology are believed to be related to better cognitive performance. For example, previous research has shown that a more efficient global functional network in terms of integration was related to better intellectual performance [32]. Therefore, as the functional brain network of children with CAE were less efficiently organized, there might be a relation between the impaired neurocognitive performance in children with CAE and the whole-brain functional network efficiency. However, in the current study no relation was found between the neurocognitive performance and global graph metrics.

\section{Study considerations}

Our study has important strengths. The study used well defined inclusion criteria for children with CAE in agreement with current ILAE standards. Moreover, the age and sex-matched controls enabled us to make reliable group-level comparisons. Furthermore, to minimize the influence of subject motion, which is an inherent problem when scanning young children, the head motion was carefully considered in both preprocessing and statistical analysis. The main limitation of this study is the relatively low sample size, although even with our small sample size we already report between-group differences as well as relations with the duration of epilepsy. However, a larger sample is required for a more in depth analysis of the relation of neurocognitive characteristics. Furthermore, it cannot be excluded that at least some of the children with CAE suffered from GSWD during the fMRI acquisition. This could have a bearing on our results, as previously it was shown that GSWD could have an effect on the graph theoretical metrics of functional networks [15]. However, since the fMRI exam lasts over 6 minutes, while absence seizures generally last a few seconds, the effects are likely to be minimal. Last, during the MRI examination 15 out of 17 children with CAE were on antiepileptic drug (AED) treatment. Since Ethosuximide and Valproic acid have been associated with neurocognitive side effects [31], [33], it might have a bearing on our results. However, since the current guidelines prescribe the use of these AED's, our results reflect children with $\mathrm{CAE}$ at this point in time. 


\section{Conclusion}

Disruptions of the functional network in terms of longer path lengths, weaker clustering and a less small-world network topology were observed in children with CAE compared to controls. Moreover, longer path lengths are related to a longer duration of CAE and a higher seizure frequency. Furthermore, cognitive performance was found to be impaired in the children with CAE, though a relation between the impaired cognitive performance and global graph metrics could not be formally established. These results indicate that less efficiently organized functional networks are a characteristic of CAE which is related to disease, and is therefore suggested to be a part of the underlying mechanism of the pathology. 


\section{References}

[1] A. T. Berg, S. Shinnar, S. R. Levy, and F. M. Testa, "Newly Diagnosed Epilepsy in Children: Presentation at Diagnosis," Epilepsia, vol. 40, no. 4, pp. 445-452, 1999.

[2] E. K. Curwood, M. Pedersen, P. W. Carney, A. T. Berg, D. F. Abbott, and G. D. Jackson, "Abnormal cortical thickness connectivity persists in childhood absence epilepsy.," Ann. Clin. Transl. Neurol., vol. 2, no. 5, pp. 456-64, 2015.

[3] Q. Li et al., "Altered resting state functional network connectivity in children absence epilepsy," J. Neurol. Sci., vol. 354, pp. 79-85, Jul. 2015.

[4] S. K. Kessler, D. Dlugos, J. Conry, D. G. Hirtz, S. L. Moshé, and P. Clark, "Pretreatment seizure semiology in childhood absence epilepsy," Neurology, vol. 89, pp. 673-679, 2017.

[5] C. Luo et al., "Altered functional connectivity in default mode network in absence epilepsy: A resting-state fMRI study," Hum. Brain Mapp., vol. 32, no. 3, pp. 438-449, 2011.

[6] R. Guerrini, “Epilepsy in children," Lancet, vol. 367, no. 9509, pp. 499-524, Feb. 2006.

[7] E. P. G. Vining and L. L. Thio, "Absence in childhood absence epilepsy," Neurology, vol. 81, no. 18, pp. 1564-1565, 2013.

[8] R. Caplan et al., "Childhood absence epilepsy: Behavioral, cognitive, and linguistic comorbidities," Epilepsia, vol. 49, no. 11, pp. 1838-1846, Nov. 2008.

[9] A. Loughman, S. C. Bowden, and W. D'Souza, "Cognitive functioning in idiopathic generalised epilepsies: A systematic review and meta-analysis," Neurosci. Biobehav. Rev., vol. 43, pp. 20-34, Jun. 2014.

[10] E. L. A. Fonseca Wald et al., "Cognitive development in absence epilepsy during long-term follow-up," Child Neuropsychol., vol. 25, no. 8, pp. 1003-1021, 2019.

[11] C. Luo et al., "Altered intrinsic functional connectivity of the salience network in childhood absence epilepsy," J. Neurol. Sci., vol. 339, no. 1-2, pp. 189-195, Apr. 2014.

[12] X. Wang, D. Jiao, X. Zhang, and X. Lin, "Altered degree centrality in childhood absence epilepsy: A resting-state fMRI study," J. Neurol. Sci., vol. 373, pp. 274-279, 2017.

[13] W. Qiu, C. Yu, Y. Gao, A. Miao, L. Tang, and S. Huang, "Disrupted topological organization of structural brain networks in childhood absence epilepsy," Sci. Rep., no. August, pp. 1-10, 2017.

[14] A. Verrotti, S. Matricardi, V. E. Rinaldi, G. Prezioso, and G. Coppola, "Neuropsychological impairment in childhood absence epilepsy: Review of the literature," J. Neurol. Sci., vol. 359, no. 1-2, pp. 59-66, 2015.

[15] W. Liao et al., "Dynamical intrinsic functional architecture of the brain during absence seizures," Brain Struct Funct, vol. 219, pp. 2001-2015, 2014.

[16] H. Onias et al., "Brain complex network analysis by means of resting state fMRI and graph analysis: Will it be helpful in clinical epilepsy?," Epilepsy Behav., vol. 38, pp. 71-80, Sep. 2014.

[17] Q. K. Telesford, J. H. Burdette, and P. J. Laurienti, "An exploration of graph metric reproducibility in complex brain networks," Front. Neurosci., vol. 7, pp. 1-9, 2013.

[18] M. Rubinov and O. Sporns, "Complex network measures of brain connectivity: Uses and interpretations," Neuroimage, vol. 52, no. 3, pp. 1059-1069, Sep. 2010.

[19] D. J. Watts and S. H. Strogatz, "Collective dynamics of 'small-world' networks," Nature, vol. 393, no. 6684, pp. 440-442, Jun. 1998.

[20] G. S. Drenthen et al., "Lower myelin-water content of the frontal lobe in childhood absence epilepsy," Epilepsia, vol. 60, no. 8, pp. 1689-1696, 2019.

[21] B. Fischl, "Automatically Parcellating the Human Cerebral Cortex," Cereb. Cortex, vol. 14, no. 1, pp. 11-22, Jan. 2004.

[22] R. S. Desikan et al., "An automated labeling system for subdividing the human cerebral cortex on MRI scans into gyral based regions of interest," Neuroimage, vol. 31, pp. 968-980, Jul. 2006.

[23] W. Liao et al., "Altered functional connectivity and small-world in mesial temporal lobe epilepsy," PLoS One, vol. 5, no. 1, pp. 27-29, 2010. 
[24] K. R. A. Van Dijk, M. R. Sabuncu, and R. L. Buckner, “The influence of head motion on intrinsic functional connectivity MRI," Neuroimage, vol. 59, no. 1, pp. 431-438, Jan. 2012.

[25] S. Klein, M. Staring, K. Murphy, M. A. Viergever, and J. Pluim, "elastix: A Toolbox for IntensityBased Medical Image Registration," IEEE Trans. Med. Imaging, vol. 29, no. 1, pp. 196-205, Jan. 2010.

[26] B. C. M. van Wijk, C. J. Stam, and A. Daffertshofer, "Comparing Brain Networks of Different Size and Connectivity Density Using Graph Theory," PLoS One, vol. 5, no. 10, p. e13701, Oct. 2010.

[27] M. A. De Reus and M. P. Van Den Heuvel, "Estimating false positives and negatives in brain networks," Neuroimage, vol. 70, pp. 402-409, 2013.

[28] G. E. P. Box and D. R. Cox, "An analysis of transformations," J. R. Stat. Soc. Ser. B, vol. 26, pp. 211-246, 1964.

[29] J. J. Bear, "The epileptic network and cognition: What functional connectivity is teaching us about the childhood epilepsies," Epilepsia, vol. 60, pp. 1491-1507, 2019.

[30] C. Park et al., "Seizure Control and Memory Impairment Are Related to Disrupted Brain Functional Integration in Temporal Lobe Epilepsy," Neuropsychiatry Clin Neurosci, vol. 29, no. 4, pp. 343-350, 2017.

[31] D. Masur et al., "Pretreatment cognitive deficits and treatment effects on attention in childhood absence epilepsy," Neurology, vol. 81, no. 18, pp. 1572-1580, 2013.

[32] M. P. Van Den Heuvel, C. J. Stam, S. Kahn, and H. E. H. Pol, "Efficiency of Functional Brain Networks and Intellectual Performance," J. Neurosci., vol. 29, no. 23, pp. 7619-7624, 2009.

[33] D. M. Ijff et al., "The Cognitive Profile of Ethosuximide in Children," Pediatr. Drugs, vol. 18, no. 5, pp. 379-385, 2016. 



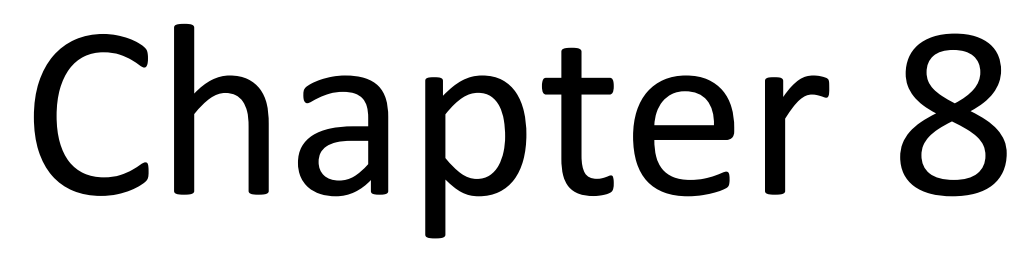

\section{Structural covariance networks relate to the severity of epilepsy with focal-onset seizures}

Gerhard S. Drenthen, Walter H. Backes, Rob P.W. Rouhl, Marielle C.G.

Vlooswijk, Marian H.J.M. Majoie, Paul A.M. Hofman, Albert P. Aldenkamp, Jacobus F.A. Jansen

NeuroImage Clin. 2018; 20:861-867. DOI: 10.1016/j.nicl.2018.09.023 


\section{Abstract}

The brains of patients with epilepsy may exhibit various morphological abnormalities, which are often not directly visible on structural MR images, as they may be focally subtle or related to a more large-scale inconspicuous disorganization of brain structures. To explore the relation between structural brain organization and epilepsy characteristics, including severity and cognitive co-morbidity, we determined structural covariance networks (SCNs). SCNs represent interregional correlations of morphologic measures, for instance in terms of cortical thickness, between various large-scale distributed brain regions.

Thirty-eight patients with focal seizures of all subtypes and 21 healthy controls underwent structural MRI, neurological, and IQ assessment. Cortical thickness was derived from the structural MRIs using FreeSurfer. Subsequently, SCNs were constructed on a grouplevel based on correlations of the cortical thicknesses between various brain regions. Individual SCNs for the epilepsy patients were extracted by adding the respective patient to the control group prior to the SCN construction (i.e. add-one-patient approach). Calculated network measures, i.e. path length, clustering coefficient and betweenness centrality were correlated with characteristics related to the severity of epilepsy, including seizure history and age at onset of epilepsy, and cognitive performance.

Stronger clustering in the individual SCN was associated with a higher number of focal to bilateral tonic-clonic seizures during life time, a younger age at onset, and lower cognitive performance. The path length of the individual SCN was not related to the severity of epilepsy or cognitive performance. Higher betweenness centrality of the left cuneus and lower betweenness centrality of the right rostral middle frontal gyrus were associated with increased drug load and younger age at onset, respectively. These results indicate that the correlations between interregional variations of cortical thickness reflect disease characteristics or responses to the disease and deficits in patients with epilepsy with focal seizures. 


\section{Introduction}

The epileptic brain often has morphological abnormalities including characteristic lesions and other subtle deviations which are not visible at radiological inspection. To understand these abnormalities, several previous studies investigated the cortical thickness in various brain regions in children and adults with epilepsy [1]-[3]. More recently, morphological brain measurements, especially cortical thickness, of various brain regions have been shown to correlate across subjects. These correlations are thought to be part of an underlying anatomical network reflecting interregional correlations of cortical thicknesses, the structural covariance network (SCN). However, whether these lesion-unspecific morphological abnormalities are related to disease characteristics of the epilepsy remains largely unknown.

The biological concept of a SCN relies on the assumption that axonally connected regions have trophic, developmental, and maturational concordances, resulting in similar variation patterns of morphology [4]. Since epilepsy has previously been associated with morphological changes, also distant to the seizure onset zone [2], [5], these type of group-level SCNs can be especially valuable in epilepsy studies, providing unique knowledge on interregional cortical associations [6]-[8]. However, since the correlations between cortical thickness values of different brain regions are usually obtained by correlating the thickness values over a group of subjects, individual changes cannot be obtained directly from the group-level SCNs.

Recently, Saggar et al. presented a method for calculating individual contribution on the group-level SCN [9]. By adding one patient (AOP) to a group of healthy controls prior to the SCN construction, the individual contribution of patients on the SCN can be measured. In the current study, this individualized method will be used to obtain individual measures of the SCN.

The current study aims to assess whether there are associations between individual SCNs and characteristics related to the severity of epilepsy, including the seizure history, age at onset, drug load, and the most common comorbidity, cognitive deterioration. To our knowledge, this is the first study that relates individual SCNs based on cortical thickness to individual epilepsy characteristics.

\section{Materials and Methods}

\section{Participants}

We included 59 participants in this study, of which 38 were clinically diagnosed with epilepsy with focal seizures of various subtypes and 21 were healthy controls. These participants have already been investigated in a number of prior studies with different MRI modalities and study objectives [10]-[15]. All subjects gave written informed consent before participation, 
and had no significant clinical MR abnormalities, as assessed by a board certified neuroradiologist. Furthermore, the following epilepsy characteristics were recorded: age at onset, drug load, seizure focus (i.e. frontal, temporal, or frontotemporal and left, right or bilateral), and the number of focal seizures during lifetime. The latter was calculated using patient records and seizure diaries [10]. The lifetime number of focal seizures with and without impairment of awareness was expressed in eight categories (0, 1-10, 11-20, 21-30, 31$40,41-50,51-100$, and $>100$ seizures), since they were more likely to occur unperceived and were, therefore, less accurately reported [10]. The lifetime number of focal to bilateral tonicclonic seizures could be extracted more accurately from the seizure diaries. Seizure focus was estimated based on EEG and seizure semiology. For all patients, no focal to bilateral tonicclonic seizures were reported in the last two weeks before the MRI acquisition, and there were no obvious differences in the behavioral markers of patients across the different seizure foci. Drug load was calculated by using the ratio of prescribed daily dose to defined daily dose [16]. For all the participants, a full-scale IQ (FSIQ) was determined using the Wechsler Adult Intelligence Scale third edition (WAIS-III) [17]. A summary of the subject characteristics is provided in Table 8.1 .

Table 8.1: Characteristics of patients with focal epilepsy and healthy controls. Variables are summarized as means \pm standard deviations for normal data or median (interquartile range) for nonnormally distributed data.

\begin{tabular}{llll}
\hline & Epilepsy & Controls & p-value \\
\hline \#Subjects & 38 & 21 & - \\
Age (in years) & $40 \pm 12$ & $40 \pm 14$ & .93 \\
Sex (Male/Female) & $20 / 18$ & $15 / 6$ & .18 \\
Age at onset (in years) & $22 \pm 13$ & - & - \\
\#FBTCS during lifetime & $5(21)$ & - & - \\
\#FSIA during lifetime & $5(7)$ & - & - \\
Drug load & $1.8 \pm 1.1$ & - & - \\
Seizure focus (F/T/FT) & $14 / 12 / 12$ & - & - \\
Seizure focus (L/R/Bi) & $15 / 9 / 14$ & - & - \\
Intelligence (FSIQ) & $96 \pm 15$ & $114 \pm 15$ & $<.01$ \\
\hline
\end{tabular}

Abbreviations: FSIQ, full-scale IQ; FBTCS, focal to bilateral tonic-clonic seizures; FSIA, focal seizures with and without impairment of awareness; $F$, frontal; $T$, temporal; $F T$, frontotemporal; L, left; $R$, right; Bi, bilateral. 


\section{MRI Acquisition}

Magnetic resonance imaging (MRI) was performed on a 3 Tesla scanner (Philips Achieva, Best, the Netherlands). T1-weighted 3D fast gradient echo images were acquired for all the participants with the following parameters: repetition time (TR) $9.91 \mathrm{~ms}$, echo time (TE) 4.6 $\mathrm{ms}$, inversion time (TI) $3 \mathrm{~s}$, flip angle $8^{\circ}$, voxel size $1 \times 1 \times 1 \mathrm{~mm}$, and matrix $256 \times 256 \times 200$.

\section{Analysis}

\section{Preprocessing}

From the T1-weighted images, the cortical thickness was determined using Freesurfer (version 5.1 [18]). The brain was parcellated into 68 cortical regions using the Desikan-Killiany atlas [19] and the mean cortical thickness was calculated for each region.

\section{Group-based SCNs}

For both patients and controls, adjacency matrices were obtained by calculating the Pearson's correlation coefficient between the cortical thicknesses of each region pair. The resulting adjacency matrix elements were represented in binary values by using a threshold to select the strongest correlations. To avoid that the statistical analysis would be driven by the total number of connections (edges) in the network, the threshold was chosen such that the networks exhibit an equal number of strongest correlations, i.e. the networks are equally sparse [20]. Negative correlations occurred fewer than $5 \%$ of the total number of correlations and were set to zero, since it is still unclear what their involvement in the network is [21]. The resulting binary adjacency matrix represents the $\mathrm{SCN}$, where a value of ' 1 ' denotes a connection between two regions and a ' 0 ' in the absence of a connection.

\section{Individual SCNs}

To extract the individual contribution of a patient on the SCN of a healthy control group, Saggar et al. introduced a so-called distance-based method [9], in which the individual contribution of a patient can be assessed by adding one patient (AOP) to the control group before calculating the adjacency matrix (Figure 8.1). With this procedure, the SCN exhibits alterations compared to the SCN obtained from just the healthy control group, which are specific for the added patient. The SCN properties are quantified in terms of graph theoretical metrics.

\section{Network analysis}

The SCNs are quantitatively described by two of the most robust and widely applied global graph metrics, the characteristic path length $(L)$, clustering coefficient $(C)$ [22] as well as a regional measure, the betweenness centrality $(B C)$ [23]. $L$ gives insight into how well 
information can spread throughout a network, while the $C$ provides a measure of local information processing. A network with strong local clustering and a short path length between nodes is considered efficiently organized. The $B C$ is a nodal graph metric that measures the number of shortest paths passing through a node, ignoring alternative paths, thereby providing insight into the influence of a node on the flow of information throughout the network. Network analysis is highly dependent on the network topology [20]. Therefore, to obtain normalized global graph measures, the global graph metrics are determined relative to the average of 1000 random networks with similar degree distribution $\left(\lambda=L / L_{\text {rand }}\right.$ and $\gamma=$ $C / C_{\text {rand }}$ ), and only networks with the same number of nodes and edges (i.e. the networks are equally sparse) will be compared. Furthermore, to decrease the occurrence of false positives and false negatives in the network, only the nodes and edges present in the network reconstructed from the healthy controls are considered in the graph analysis (i.e. group thresholding) [24]. The reconstructed network is obtained by calculating the Pearson correlation coefficients for the control group and selecting only the statistically significant positive correlations $(p<.05)$ as edges. Subsequently, since the optimal number of edges in a SCN is unknown and to prevent that weakly correlated connections are included (Type I errors) and that those with a high correlation are excluded (Type II errors), the number of edges in each network is varied such that the SCNs are $60-90 \%$ sparse, with intervals of $1 \%$. Networks sparser than $90 \%$ would lead to disconnected nodes, hindering the graph analysis.
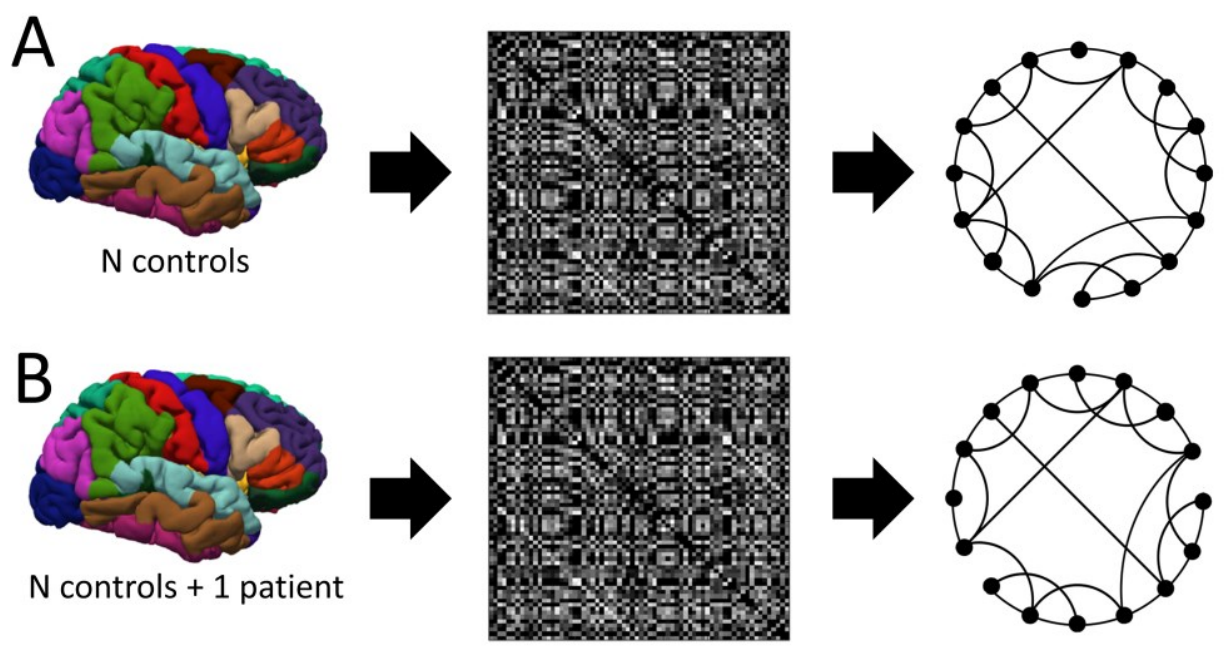

Figure 8.1: A) SCN estimation procedure for healthy controls, from the control subjects an adjacency matrix is obtained using Pearson's correlation. The adjacency matrix represents a graph. B) by adding a specific patient to the control group, the resulting adjacency matrix and graph exhibit some patientspecific alterations. 


\section{Statistical analysis}

Previously, cortical thickness has been shown to decrease with age [25], to differ between males and females [26], and to scale with the total intracranial volume (TIV) [27]. Therefore, for each of the 68 regions, the cortical thickness values were corrected for the effects of age, sex and TIV via linear regression models [28]. Differences in age, FSIQ, and corrected regional cortical thickness values were assessed with the independent samples t-test. Differences in sex were assessed with the chi-squared test of independence.

To investigate whether the cortical thickness values or the global graph metrics ( $\lambda$ and $\gamma$ ) are dependent on the location of the seizure focus (frontal, temporal or frontotemporal as well as left, right or bilateral), one-way ANOVA tests are performed. The regional corrected cortical thickness and the graph metrics are used as dependent variables in the multivariate regression analysis. Two separate sets of groups are defined as: 1) patients with a frontal, a temporal or a frontotemporal seizure focus, and 2) patients with a left, a right or a bilateral seizure focus. The false discovery rate (FDR) is used to correct for comparisons over multiple brain regions.

The Pearson's correlation coefficient was used to correlate $\lambda$ and $\gamma$ with FSIQ and drug load, while the correlation of the non-normally distributed variables focal to bilateral tonicclonic seizures during lifetime, age at onset and focal seizures with and without impairment of awareness during lifetime with $\lambda$ and $\gamma$ was assessed using the non-parametric Spearman's $\rho$. Thereafter, a multivariate forward stepwise regression was performed to predict $\lambda$ and $\gamma$ from the variables that previously showed a significant association in the univariate analysis. Furthermore, to assess whether the relation was significantly associated with the seizure focus, four covariates (frontal focus yes/no, temporal focus yes/no, left focus yes/no and right focus yes/no) were added to the regression model.

Multivariate forward stepwise regression analysis was performed to investigate if $B C$ was associated with the FSIQ, drug load, focal to bilateral tonic-clonic seizures during lifetime, age at onset and focal seizures with and without impairment of awareness during lifetime, while accounting for the different seizure foci. To this end, the $B C$ of each node was predicted from one of the clinical characteristics and four added seizure focus covariates (frontal focus yes/no, temporal focus yes/no, left focus yes/no and right focus yes/no). The rate FDR was used to correct for comparisons over multiple brain regions.

The non-normally distributed variables were log-transformed prior to the regression analysis. To assess differences between groups, the non-parametric permutation test [8], with 1000 permutations, was used.

The individual SCN analysis, the graph analysis and the statistical analysis are all performed using MATLAB (version R2014b) software. All associations investigated between graph metrics and epilepsy variables were evaluated with regard to robustness to network variations by varying the sparsity value $(60-90 \%)$.

Statistical significance was inferred at $p<.05$. 


\section{Results}

\section{Patient characteristics}

No significant differences between the patients with epilepsy and healthy controls are reported for age and gender $(p>.10)$. However, the patients with epilepsy had significantly lower FSIQ scores compared to the healthy controls (96 \pm 15 vs $114 \pm 15, p<.01)$. The patients showed cortical thinning in the postcentral gyrus and posterior cingulate in the left hemisphere, and the insula in the right hemisphere (respectively, mean \pm SD in $\mathrm{mm}, 2.79 \pm .16$

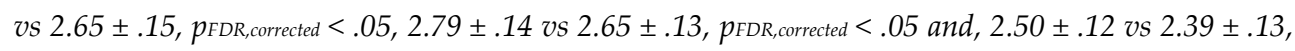
$\left.p_{F D R, \text { corrected }}<.05\right)$. These three regions are highlighted on an inflated cortical surface in Figure 8.2. Cortical thickness did not differ significantly for the various seizure focus localizations $\left(p_{F D R, \text { corrected }}>.14\right)$.

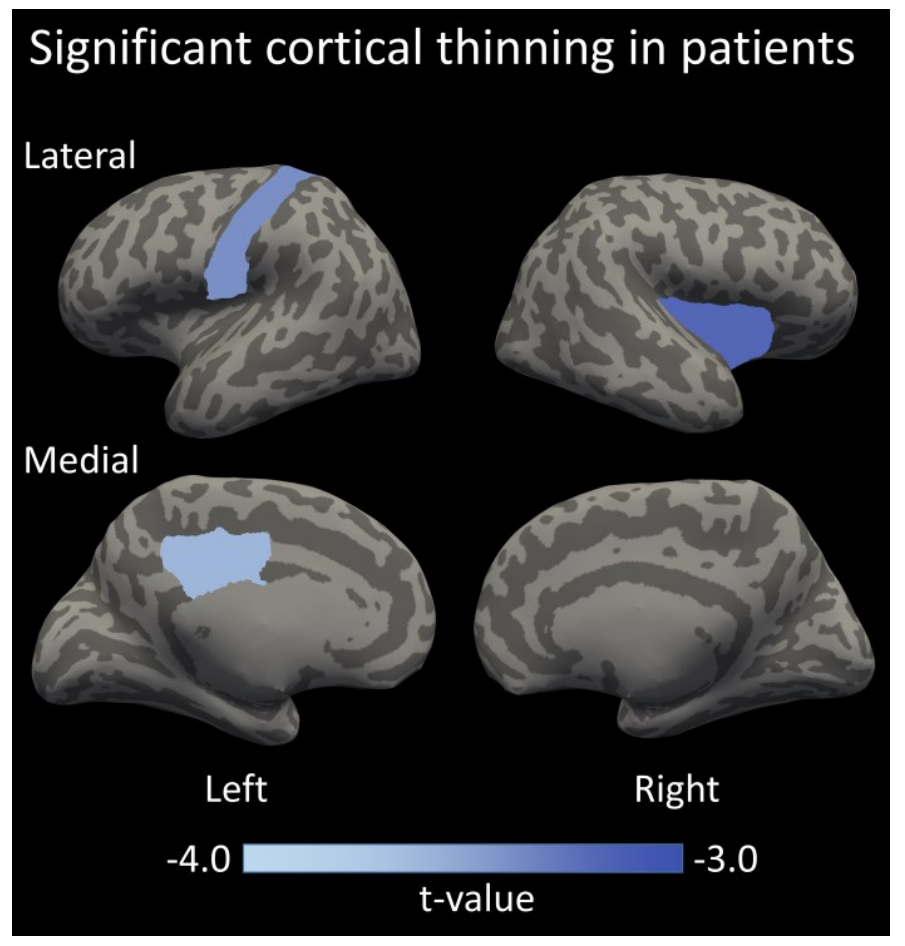

Figure 8.2: The three cortical regions, the postcentral gyrus and posterior cingulate in the left hemisphere, and the insula in the right hemisphere, that show a significantly decreased cortical thickness in patients compared to healthy controls. 


\section{Severity of epilepsy and cognitive comorbidity}

In Figure $8.3, \gamma$ and $\lambda$ are shown as a function of sparsity and significant associations between the two graph metrics and the clinical characteristics can be denoted. A positive correlation was found in the patient group for $\gamma$ and the number of focal to bilateral tonic-clonic seizures during lifetime over the analysed sparsity range, indicating that an increased $\gamma$ is associated with more seizures.

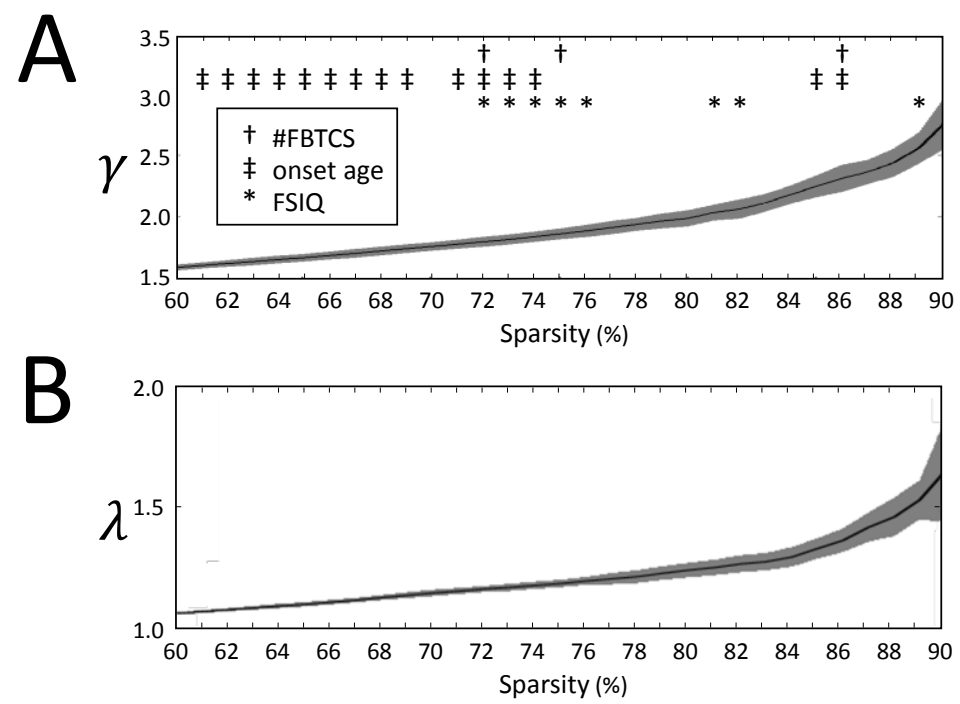

Figure 8.3: The mean and standard deviation of the network measures $(A) \gamma$ and $(B) \lambda$ are shown as a function of sparsity. Significant correlations between network measures and epilepsy characteristics: tpositive correlation with number of focal to bilateral tonic-clonic seizures during lifetime łnegative correlation with onset age *negative correlation with FSIQ.

A negative correlation was found in the patient group between $\gamma$ and age at onset of epilepsy over the analysed sparsity range, indicating that an increased $\gamma$ is associated with a younger age at onset of epilepsy. A scatter plot showing the relation between $\gamma$ and age at onset of epilepsy for networks that are $72 \%$ sparse is shown in Figure 8.4A.

Neither consistent, nor significant associations were found between the network metrics $\gamma$ and $\lambda$ and either the focal seizures with and without impairment of awareness or the drug load.

A negative correlation was found for $\gamma$ and FSIQ over the analysed sparsity range, indicating that an increased $\gamma$ is associated with a lower FSIQ. A scatter plot showing the relation between $\gamma$ and FSIQ for networks that are 72\% sparse is shown in Figure 8.4B. 

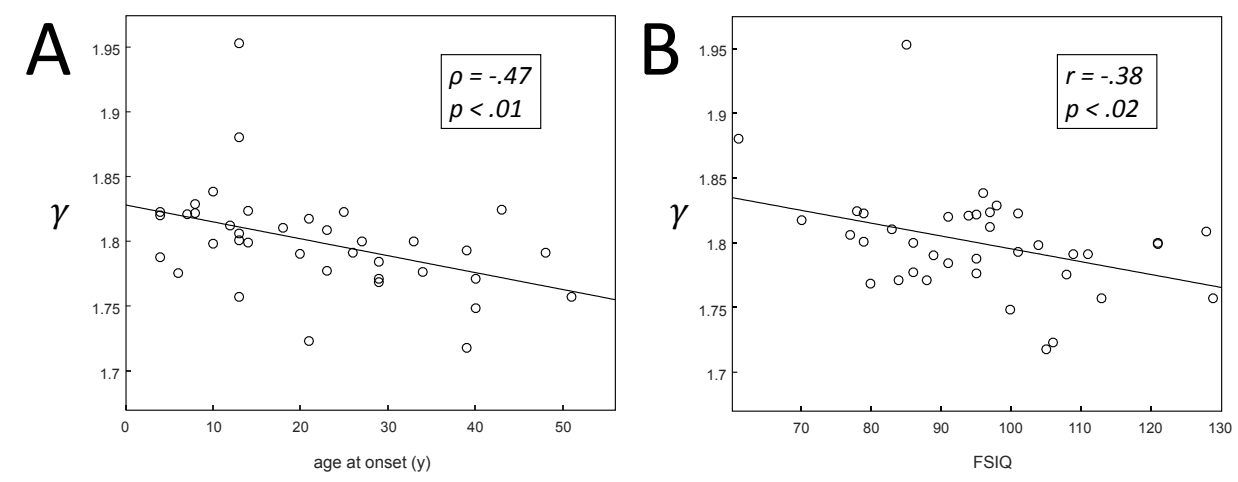

Figure 8.4: Scatter plot of $\gamma$ vs (A) age at onset and (B) FSIQ for a sparsity level of $72 \%$. Linear least squares lines are fitted through the data points for visualization.

The correlation between $\lambda$ and either the number of focal to bilateral tonic-clonic seizures during lifetime, age at onset or the FSIQ showed no such consistent correlation over the analysed sparsity range.

Since the correlations between $\gamma$ and focal to bilateral tonic-clonic seizures during lifetime, age at onset as well as FSIQ were all significant for $72 \%$ sparse networks, a multivariate stepwise linear regression analysis was performed to predict the $\gamma$ for $72 \%$ sparse networks using focal to bilateral tonic-clonic seizures during lifetime, age at onset, FSIQ and two variables representing the seizure focus. As a result, both focal to bilateral tonic-clonic seizures during lifetime $(\beta=.37, p<.05)$ and FSIQ $(\beta=-.31, p<.05)$ were entered into the regression model $\left(F(2,35)=5.48, p<.01, R^{2}=.24\right)$, and both added significantly to the prediction. Age at onset and the seizure focus variables $(p>.05)$ were excluded from the regression model.

The multivariate regression analyses predicting $B C$ from the clinical characteristics revealed significant associations $\left(p_{F D R_{-} \text {corrected }}<.05\right)$ between drug load and the $B C$ of the orbitalis, inferior temporal gyrus, cuneus and supramarginal gyrus of the left hemisphere and the postcentral gyrus, inferior temporal gyrus, cuneus and rostral middle frontal gyrus of the right hemisphere (Figure 8.5A). Moreover, age at onset of epilepsy was associated with the $B C$ of the cuneus and temporal pole of the left hemisphere and the medial orbitofrontal cortex and rostral middle frontal gyrus of the right hemisphere (Figure $8.5 \mathrm{~B}$ ). The seizure foci variables were not added to any of the regression models using the stepwise approach

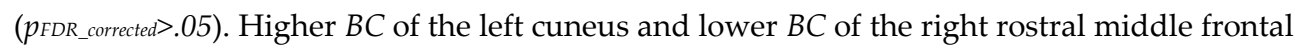
gyrus was associated with both increased drug load and younger age at onset of epilepsy. 


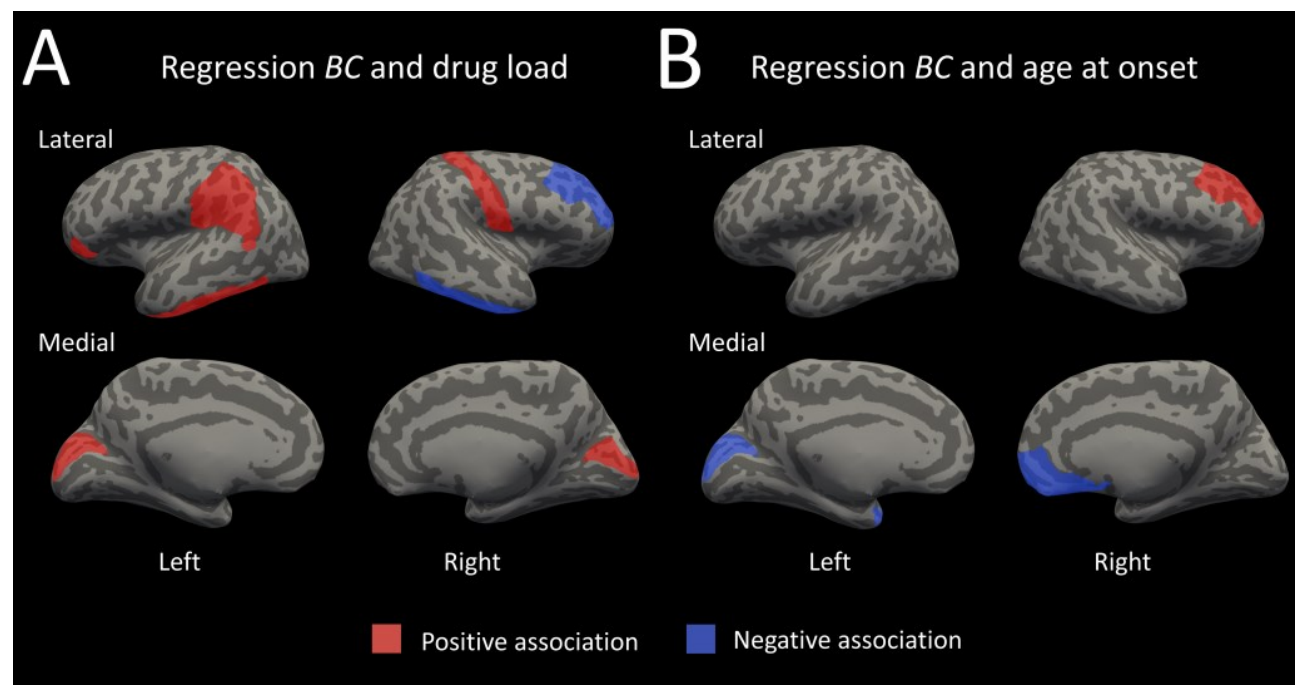

Figure 8.5: The brain regions that show a significant (positive/negative) relation between $B C$ and either drugload (A) or age at onset (B) are highlighted. The $B C$ of the left cuneus and right rostral middle frontal gyrus are related to both drug load and age at onset. $B C$, betweeness centrality.

\section{Group-level differences}

Using the non-parametric permutation test, no significant differences were found in any of the graph metrics between the patients and controls $(p>.10)$.

\section{Discussion}

\section{Current findings}

The current study aimed to investigate the association between network measures of cortical thickness, in terms of individual SCNs, and clinical characteristics of patients with epilepsy with focal seizures. For the first time, it was shown that properties of SCN are correlated to characteristics of epilepsy severity. Stronger clustering within the SCN was found to be associated with (i) an increased number of focal to bilateral tonic-clonic seizures during lifetime, (ii) a younger age at onset of epilepsy and (iii) lower cognitive performance scores. Furthermore, an increased number of focal to bilateral tonic-clonic seizures during lifetime and a lower cognitive performance score were found to have the strongest association with clustering. No significant differences in terms of path length were found between the patient and control group. However, a lower $B C$ of the left cuneus and right rostral middle frontal gyrus was shown to be associated with increased drug load and a younger age at onset of epilepsy. 


\section{Severity of epilepsy and the SCN}

Our results show a relation between stronger clustering of the cortical network and higher number of focal to bilateral tonic-clonic seizures during lifetime. Previously, an increased seizure frequency was reported to be related to widespread decrease of functional connectivity in hot-water epilepsy [29] and also functional disintegration of the default mode network in temporal lobe epilepsy (TLE) [30]. Prior cohort studies in children with epilepsy suggested that early changes of functional brain organization are followed by changes of structural connectivity [2], [3]. Therefore, the decrease (or disintegration) of functional connectivity might lead to changes in the cortical network in epilepsy. Furthermore, the notion of early changes in brain organization is in agreement with the relation with age at onset as found in the current study. In this study, stronger clustering is associated with a younger age at onset, indicating that the younger brain might already start to develop morphological adaptions in a large-scale network. This is further supported by a relation found in TLE between an increased number of seizures and neocortical atrophy [31]. However, based on the current cross-sectional study design no such causal relation can be drawn formally. Alternatively, segregation of the brain network into several clusters has been implied to be a containment mechanism for activity, such as epileptic seizures [30]. Therefore, the reported association could also be a compensatory mechanism, attempting to limit the effect of the seizures.

Increased drug load and younger age at onset of epilepsy are related to a lower $B C$ of the right rostral middle frontal gyrus. Possibly, this locally decreased $B C$ is part of a containment mechanism to isolate seizures with a frontal focus. Additionally, a higher $B C$ of the left cuneus was found to be associated to increased severity of epilepsy (higher drug load and younger age at onset). Previously, the cortex of the cuneus was found to be thinner in patients with TLE and stronger cognitive impairment [32]. Therefore, the observed effect might be related to changes in the cortical thickness of epilepsy patients. However, since no relation was found for a frontal or temporal seizure focus, this interpretation and its relation to the seizure focus should be investigated in more detail with other studies.

\section{Cognitive performance and the SCN}

In this study, decreased cognitive performance was found to be associated with an increase in clustering of the cortical network. Two previous studies, focusing on altered network efficiency with other MRI techniques, were performed with the same (sub)set of subjects as the current study [12], [14]. Using diffusion tensor imaging (DTI) to construct structural networks, Vaessen et al. found that both weaker clustering and longer path lengths were related to lower cognitive performance. Furthermore, using functional MRI (fMRI) Vlooswijk et al. reported a significant association between weaker clustering and lower cognitive performance. Interestingly, the results reported in this study show an opposite relation 
between cognitive performance and clustering in the cortical network. Previously, SCNs were found to only partly (35-40\%) reflect the fiber connections obtained using DTI [21]. Furthermore, since epilepsy has been associated with morphological changes [2], [5], the SCNs might provide unique knowledge on interregional cortical associations in epilepsy, and therefore provide information which is intrinsically different from DTI and fMRI. The relationship between the network measures derived from different MRI techniques (SCN, fMRI and DTI) still needs to be elucidated in epilepsy.

\section{Group-level differences}

Cortical thinning in patients with epilepsy was observed in several cortical areas. Reduced cortical thickness was previously reported in TLE [33], [34], and is potentially related to excitotoxic damage from seizures [35]. The non-parametric permutation test did not yield any differences for either the degree of clustering or the path length between patients with epilepsy and controls. The lack of differences between the two groups implies that the global characteristics of the SCN of patients with epilepsy is not significantly different from the healthy control group, which might be due to variations in the SCN between patients. This is in contrast to previous SCN studies in epilepsy [6]-[8], which might be explained by differences in patient populations, analysis method and sample size between our study and those studies. The current study shows that these variations in SCN between epilepsy patients reflect to some extent the epilepsy characteristics.

\section{Study considerations}

The complex interactions between morphological features (e.g. cortical thickness) are still not completely understood at a cellular level. Furthermore, whether the SCN resemble patterns of white matter connections or are more related to functional connectivity still needs to be resolved. However, in the current and previous studies it has been shown that the SCNs can be a valuable metric in epilepsy research [6]-[8]. The location of the epileptic focus seems not of influence in the current study, however, larger study samples for the different foci are prompted for to be more definite on this matter. 


\section{Conclusion}

The clustering of the cortical SCN is associated with the severity of epilepsy in terms of a higher number of focal to bilateral tonic-clonic seizures during lifetime, younger age at onset and lower cognitive performance. These results indicate that the correlations between interregional variations of cortical thickness reflect disease characteristics or responses to the disease and deficits in patients with epilepsy with focal seizures. 


\section{References}

[1] E. Widjaja, S. Z. Mahmoodabadi, O. C. Snead, A. Almehdar, and M. Lou Smith, "Widespread cortical thinning in children with frontal lobe epilepsy," Epilepsia, vol. 52, no. 9, pp. 1685-1691, 2011.

[2] G. M. Overvliet et al., "Early onset of cortical thinning in children with rolandic epilepsy," NeuroImage Clin., vol. 2, pp. 434-439, 2013.

[3] R. M. H. Besseling et al., "Delayed convergence between brain network structure and function in rolandic epilepsy," Front. Hum. Neurosci., vol. 8, p. 704, 2014.

[4] B. C. Bernhardt, Z. Chen, Y. He, A. C. Evans, and N. Bernasconi, "Graph-Theoretical Analysis Reveals Disrupted Small-World Organization of Cortical Thickness Correlation Networks in Temporal Lobe Epilepsy," Cereb. Cortex, vol. 21, no. 9, pp. 2147-2157, Sep. 2011.

[5] B. C. Bernhardt et al., "Preferential susceptibility of limbic cortices to microstructural damage in temporal lobe epilepsy: A quantitative T1 mapping study," Neuroimage, vol. 182, pp. 294-303, 2017.

[6] B. C. Bernhardt, S. Hong, A. Bernasconi, and N. Bernasconi, "Imaging structural and functional brain networks in temporal lobe epilepsy," Front. Hum. Neurosci., vol. 7, p. 624, 2013.

[7] E. K. Curwood, M. Pedersen, P. W. Carney, A. T. Berg, D. F. Abbott, and G. D. Jackson, "Abnormal cortical thickness connectivity persists in childhood absence epilepsy.," Ann. Clin. Transl. Neurol., vol. 2, no. 5, pp. 456-64, 2015.

[8] C. L. Yasuda et al., "Aberrant topological patterns of brain structural network in temporal lobe epilepsy," Epilepsia, vol. 56, no. 12, pp. 1992-2002, Nov. 2015.

[9] M. Saggar et al., "Estimating individual contribution from group-based structural correlation networks.," Neuroimage, vol. 120, pp. 274-284, Oct. 2015.

[10] M. C. G. Vlooswijk et al., "Memory processes and prefrontal network dysfunction in cryptogenic epilepsy," Epilepsia, vol. 52, no. 8, pp. 1467-1475, 2011.

[11] J. F. A. Jansen et al., "Quantitative MR and cognitive impairment in cryptogenic localisation-related epilepsy," Epileptic Disord., vol. 16, no. 3, pp. 318-327, 2014.

[12] M. J. Vaessen et al., "White matter network abnormalities are associated with cognitive decline in chronic epilepsy," Cereb. Cortex, vol. 22, no. 9, pp. 2139-2147, 2012.

[13] M. C. G. Vlooswijk et al., "Functional connectivity and language impairment in cryptogenic localization-related epilepsy.," Neurology, vol. 75, no. 5, pp. 395-402, Aug. 2010.

[14] M. C. G. Vlooswijk et al., "Loss of network efficiency associated with cognitive decline in chronic epilepsy," Neurology, vol. 77, no. 10, pp. 938-944, Sep. 2011.

[15] J. F. A. Jansen et al., "White matter lesions in patients with localization-related epilepsy.," Invest. Radiol., vol. 43, no. 8, pp. 552-558, Aug. 2008.

[16] M. W. Lammers, Y. A. Hekster, A. Keyser, H. Meinardi, W. O. Renier, and H. Lier, "Monotherapy or Polytherapy for Epilepsy Revisited: A Quantitative Assessment," Epilepsia, vol. 36, no. 5, pp. 440446, May 1995.

[17] D. Wechsler, "Manual for the Wechsler Adult Intelligence Scale, thrid edition," 1997.

[18] B. Fischl and A. M. Dale, "Measuring the thickness of the human cerebral cortex from magnetic resonance images," Proc. Natl. Acad. Sci., vol. 97, no. 20, pp. 11050-11055, Sep. 2000.

[19] R. S. Desikan et al., "An automated labeling system for subdividing the human cerebral cortex on MRI scans into gyral based regions of interest," Neuroimage, vol. 31, pp. 968-980, Jul. 2006.

[20] B. C. M. van Wijk, C. J. Stam, and A. Daffertshofer, "Comparing Brain Networks of Different Size and Connectivity Density Using Graph Theory," PLoS One, vol. 5, no. 10, p. e13701, Oct. 2010. 
[21] G. Gong, Y. He, Z. J. Chen, and A. C. Evans, “Convergence and divergence of thickness correlations with diffusion connections across the human cerebral cortex," Neuroimage, vol. 59, pp. 1239-1248, Jan. 2012.

[22] D. J. Watts and S. H. Strogatz, "Collective dynamics of 'small-world' networks," Nature, vol. 393, no. 6684, pp. 440-442, Jun. 1998.

[23] L. Freeman, "A Set of Measures of Centrality Based on Betweenness," Sociometry, vol. 40, pp. 3541, 1977.

[24] M. A. De Reus and M. P. Van Den Heuvel, "Estimating false positives and negatives in brain networks," Neuroimage, vol. 70, pp. 402-409, 2013.

[25] V. A. Magnotta et al., "Quantitative in vivo measurement of gyrification in the human brain: changes associated with aging," Cereb. Cortex, vol. 9, no. 2, pp. 151-160, 1999.

[26] E. R. Sowell et al., "Sex Differences in Cortical Thickness Mapped in 176 Healthy Individuals between 7 and 87 Years of Age," Cereb. Cortex, vol. 17, no. 7, pp. 1550-1560, Jul. 2007.

[27] K. Im, J. M. Lee, O. Lyttelton, S. H. Kim, A. C. Evans, and S. I. Kim, “Brain size and cortical structure in the adult human brain," Cereb. Cortex, vol. 18, no. 9, pp. 2181-2191, 2008.

[28] G. Sanabria-Diaz et al., "Surface area and cortical thickness descriptors reveal different attributes of the structural human brain networks," Neuroimage, vol. 50, no. 4, pp. 1497-1510, 2010.

[29] R. D. Bharath et al., "Seizure frequency can alter brain connectivity: Evidence from resting-state fmri," Am. J. Neuroradiol., vol. 36, no. 10, pp. 1890-1898, 2015.

[30] L. Douw et al., "Dissociated multimodal hubs and seizures in temporal lobe epilepsy," Ann. Clin. Transl. Neurol., vol. 2, no. 4, pp. 338-352, Apr. 2015.

[31] A. C. Coan et al., "Frequent seizures are associated with a network of gray matter atrophy in temporal lobe epilepsy with or without hippocampal sclerosis," PLoS One, vol. 9, no. 1, p. e85843, 2014.

[32] K. Dabbs, J. Jones, M. Seidenberg, and B. Hermann, "Neuroanatomical correlates of cognitive phenotypes in temporal lobe epilepsy," Epilepsy Behav., vol. 15, no. 4, pp. 445-451, 2009.

[33] S. S. Keller and N. Roberts, "Voxel-based morphometry of temporal lobe epilepsy: An introduction and review of the literature," vol. 49, no. 5, pp. 741-757, 2008.

[34] B. C. Bernhardt, N. Bernasconi, L. Concha, and A. Bernasconi, "Cortical thickness analysis in temporal lobe epilepsy: reproducibility and relation to outcome," Neurology, vol. 74, no. 22, pp. 1776-1784, Jun. 2010.

[35] N. Kemmotsu et al., “MRI Analysis in Temporal Lobe Epilepsy: Cortical Thinning and White Matter Disruptions Are Related to Side of Seizure Onset," Epilepsia, vol. 52, no. 12, pp. 2257-2266, 2011. 

Chapter 9

General Discussion 


\section{Outline}

Epilepsy is no longer thought to be merely a disorder of the gray matter, but it rather affects the complex interactions of different cortical regions connected through axon bundles in the white matter. A vital component to establish these interactions is the myelin sheath, which previously has only been scarcely considered in epilepsy [1], [2]. Therefore, in this thesis several clinical and methodological studies were implemented. The first aim of this thesis was to develop dedicated quantitative MR image techniques, focussed on image acquisition and analysis for the myelin content and cerebral networks in children with childhood absence epilepsy (CAE). The second aim was to gain additional insights into the underlying neurobiological substrate of epilepsy, with a particular interest in myelin and networks. The obtained results will be discussed in two main sections concerning (i) dedicated myelinspecific analysis techniques and (ii) involvement of myelin and networks in epilepsy.

Whereas, chapter 2 showed that there is solid evidence from the literature that the myelin content is involved in epilepsy and/or epileptic seizures, myelin-specific quantitative imaging studies in human epilepsy patients are currently scarce. Myelin-specific imaging studies might provide new avenues for understanding the underling mechanisms involved in epilepsy, epileptic seizures and their impact on brain development and the corresponding behavioural and cognitive effects. Improving techniques for adequate myelin-specific imaging are essential, and thus methodological chapters 3 and 4 reported on improvements in acquisition and analysis strategies for myelin imaging. Subsequently, in the next two chapters (5 and 6) myelin imaging was applied in children with CAE and healthy controls. Chapters 7 and 8 focussed on the involvement of cerebral networks in epilepsy, with a particular focus on relations with the severity of epilepsy and its comorbidities.

\section{Dedicated myelin-specific analysis techniques}

\section{Current findings}

In vivo myelin content in the research described in this thesis was estimated by magnetic resonance imaging (MRI) using the widely applied analysis of multi-echo T2-relaxometry [3]. Most commonly in literature, the non-negative least squares (NNLS) algorithm is used to reconstruct the measured signal from an overcomplete set of T2 relaxation curves, the socalled dictionary [4]. However, the NNLS has a high computational complexity, which limits the number of elements in the dictionary. Therefore, a modified version of the orthogonal matching pursuit (OMP) was presented as an alternative analysis method for estimation of the myelin-water content (chapter 3). The OMP has a lower computational complexity, which allows for a higher resolution dictionary as well as running multiple instances of the algorithm in the same computational time for the NNLS. The OMP 
algorithm was validated using simulations and phantom data, which indicated that OMP provides a more accurate measure of myelin-water compared to the NNLS for different signal-to-noise ratios. These findings were corroborated by in vivo results from healthy subjects, suggesting that the OMP may be a useful tool in future patient studies. For OMP validation, a single-slice multi-spin echo (MSE) sequence was used, which is considered a reference method for myelin-water imaging [3]. However, the MSE sequence is relatively slow and has a poor spatial coverage (single slice), limiting its usefulness in clinical studies. Therefore, most recent myelin-water studies employ fast 3D gradient and spin-echo (GRASE) acquisition techniques with a whole brain coverage [5]-[8]. Although chapter 3 also showed the applicability of the OMP algorithm in a 3D GRASE acquisition, the performance and reproducibility for fast acquisition methods remain undetermined. In chapter 4 the applicability and reproducibility of both algorithms (OMP and NNLS) was assessed for a newly developed 2D multi-slice GRASE acquisition covering the whole brain, with varying acceleration factors for faster acquisition. First of all, it was shown that whole brain myelin-water was feasible within an acquisition time of approximately 7 minutes. Furthermore, for the NNLS, the myelin-water values showed good overall reproducibility. The reproducibility of myelin-water estimation has a direct implication on the applicability in clinical studies, expressing the minimal detectable difference between subjects.

\section{Subject motion}

Subject motion is an inherent problem for all patients, especially when scanning young children. The most intuitive method to limit subject motion is to reduce the scan time. While most previous myelin-water studies focussed on 3D scanning methods, multi-slice (2D) techniques generally have shorter acquisition times. Therefore, in chapter 4 , a fast 2D multislice GRASE sequence for myelin estimation was designed. However, due to the sliceselective excitation, a post-acquisition correction for imperfect slice profiles was needed [9][11]. For the first time, it was shown that multi-slice acquisition for myelin-water estimation including a post-acquisition correction for imperfect slices yields reproducible myelin measures. Furthermore, it was shown that multi-slice imaging is in good agreement with the reference method for myelin-water imaging [3]. The multi-slice GRASE sequence proposed in chapter 4 is roughly twice as fast compared to the 2D CPMG sequence previously proposed by Akhondi-Asl et al. [11], as well as other 3D GRASE methods [5]-[8]. Although, it should be noted that compared to alternatives 3D sequences the spatial coverage of multislice GRASE is reduced (spatially under sampled) by $20 \%$ due to the slice gap of $1 \mathrm{~mm}$. Next to the faster acquisition, the multi-slice acquisition is also less susceptible to subject motion compared to $3 \mathrm{D}$ acquisition, where motion can corrupt the entire image volume. While subject motion cannot be prevented with a $2 \mathrm{D}$ acquisition, movement does not compromise the entire dataset. 


\section{Implications and directions for future research}

The proposed 2D acquisition technique for myelin-water estimation was shown to be in good agreement with the reference method, namely that it is sensitive and specific to the myelin content. Furthermore, the reproducibility of the fast myelin-water image acquisition was shown to be able to detect differences of about $2 \%$ absolute myelin-water content in $95 \%$ of the observations (chapter 4 ). For comparison, previous clinical studies investigating white matter myelin abnormalities in multiple sclerosis typically reported a difference of approximately $2-5 \%$ in absolute myelin-water content compared to controls [12]-[14]. However, in epilepsy there are likely more subtle differences in myelin-water content in comparison to multiple sclerosis. For example, in chapter 5 differences of approximately $1 \%$ absolute myelin-water content were observed compared to healthy controls. Therefore, prior to individual clinical use, further improvements and validation (e.g. comparisons with histological measures) are required, since myelin imaging on an individual subject level remains challenging. However, it was shown here that group differences can already be detected with relatively small sample sizes in clinically feasible scanning times.

\section{Involvement of myelin and networks in epilepsy}

\section{Myelin}

The myelin sheath is one of the most characteristic components of the white matter. In chapter 2 the current literature on the involvement of myelin in epilepsy was reviewed. While much evidence exists from preclinical and histological studies, in vivo imaging studies in patients with epilepsy are currently scarce.

In chapter 5, it was shown that the myelin-water content in children with CAE differs from healthy controls. More specifically, in the frontal lobe a widespread lower myelinwater content was observed in children with CAE. Interestingly, when assessing the diffusion MRI-derived fractional anisotropy (FA), which reflects the directional preference of water molecules that move in an orientationally hindered way and is often used as a pseudo-marker of myelin content, no significant differences were observed (Table 9.1). This indicates that the FA is not specific to only the myelin content, and that myelin-water imaging provides unique and additional information on the myelin content [15]. This further emphasizes the relevance of myelin-specific scans in patients with epilepsy, also since myelin is an important substance during the vulnerable stages of childhood development. 
Table 9.1. MWF and FA values in the 5 lobes for children with CAE and controls. Note that the uncorrected FA-values are shown, without adjustment for age and sex. The $p$-value denotes the significance level of the group difference obtained with the regression model, while correcting for the effects of age and sex.

\begin{tabular}{lllllll}
\hline & \multicolumn{3}{l}{ MWF } & & \multicolumn{2}{l}{ FA } \\
\cline { 2 - 7 } & CAE $(\%)$ & Controls $(\%)$ & p-value & CAE (-) & Controls (-) & p-value \\
\hline Frontal & $7.5 \pm 1.1$ & $8.5 \pm 1.1$ & .02 & $.32 \pm .01$ & $.33 \pm .02$ & .12 \\
Parietal & $9.3 \pm 1.2$ & $9.9 \pm 1.1$ & .22 & $.33 \pm .03$ & $.33 \pm .02$ & .60 \\
Occipital & $9.2 \pm 1.2$ & $9.7 \pm 1.2$ & .38 & $.25 \pm .07$ & $.28 \pm .06$ & .18 \\
Temporal & $6.5 \pm 0.8$ & $7.0 \pm 0.7$ & .09 & $.30 \pm .03$ & $.32 \pm .02$ & .06 \\
Insular & $10.3 \pm 1.7$ & $11.1 \pm 1.8$ & .26 & $.41 \pm .08$ & $.39 \pm .09$ & .44 \\
\hline
\end{tabular}

Abbreviations: MWF, myelin-water fraction; FA, fractional anisotropy; CAE, childhood absence epilepsy.

While myelin-water imaging provides markers specific to the myelin content, it does not provide information on the connectivity of white matter fibers directly. Therefore, diffusion MRI remains an important technique to characterize the axonal bundles, which are an important aspect of the neuronal network. In chapter 6, both the diffusion MRI and myelinwater imaging were combined to evaluate the axon-specific myelin development from childhood (7-12y) into adulthood (18-32y). Established patterns of myelination were observed, for example, tracts connecting central regions were shown to be myelinated in an earlier stage compared to the peripheral regions [16]. Subsequently, the applicability of a diffusion-MRI derived axonal atlas was tested in a sample of children with CAE. Based on the atlas, distinct cross-sectional differences between early and late developing tracts were observed compared to controls. Therefore, besides a lower myelin content in the frontal lobe compared to controls, CAE is also characterized by altered patterns of myelination.

\section{Myelin-weighted networks}

Besides determination of the axonal-specific myelin development, the myelin content could also provide additional information in graph theoretical analysis of the structural network. Myelin content is closely related to the conduction velocity of electrical signals in the brain [17], as such weighing the axonal tracts reconstructed using diffusion MRI can provide a more comprehensive picture of the structural brain network. To study the potential benefits of axon-specific myelin in structural network analysis, the structural network of children with CAE and healthy controls was quantified by graph metrics here. Two weights were considered, the FA and the myelin-water fraction (MWF). The overall efficiency of both networks was calculated in terms of the small-worldness parameter. It was found that when regarding the myelin-water as weight, the networks of children with CAE were significantly less efficiently organized (Figure 9.1A). No significant differences between the groups were 
found for the FA weighted networks (Figure 9.1B). This indicates that the myelin provides information that is more specific to CAE compared to the FA. Combined, these results and the results from chapters 2, 5 and 6 show that myelin is an important component to consider in epilepsy, especially for children.
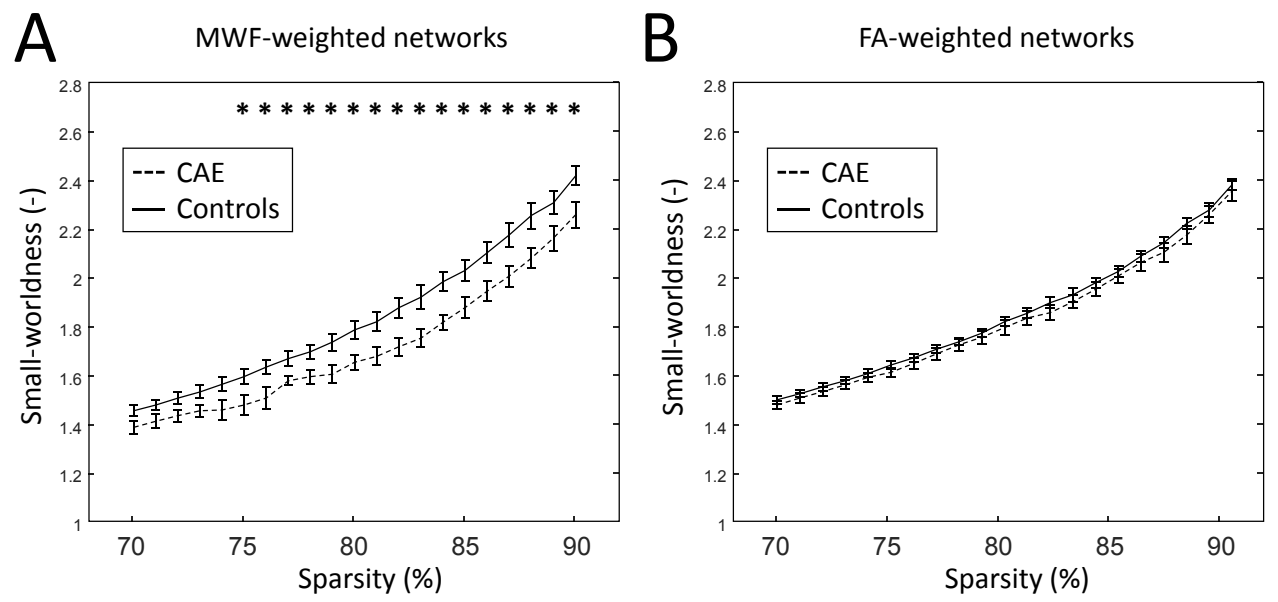

Figure 9.1: Small-worldness of A) MWF-weighted and B) FA-weighted structural networks as a function of sparsity. Mean and standard errors for children with CAE (dashed) and controls (solid) are shown. Asterisks indicate a significant difference between the two groups ( $p<.05)$. MWF, myelinwater fraction; FA, fractional anisotropy; CAE, childhood absence epilepsy.

\section{Networks related to epilepsy}

Differences between children with CAE and controls are indicative of abnormalities that, in part, reflect the disease. However, relationships between imaging metrics and either the neurocognitive performance or epilepsy characteristics provide more comprehensive information. In the children with CAE, no definite associations were found between neurocognition/epilepsy characteristics and myelin-water content (chapter 5). In chapter 7 and 8, networks based on the complex interactions of cortical activation patterns and morphology were calculated, with a specific focus on the relation to epilepsy and its comorbidities.

Chapter 7 showed that the complex interactions of cortical activation patterns, i.e. the global functional network, of children with CAE is altered compared to controls, revealing a less efficiently organized network. Furthermore, a less optimal network organization was found to be associated with a longer duration of CAE, indicating that less efficient networks are an additional feature of the pathophysiology. Together, this indicates that suffering from $\mathrm{CAE}$ for a longer time is associated with a more abnormal state of the functional network. 
Moreover, previous research has shown that a more efficient global functional network in terms of integration was related to better intellectual performance [18]. Since the functional brain network of children with CAE were less efficiently organized, this suggests a relation between the impaired neurocognitive performance and the functional network efficiency.

Besides the interregional correlations of brain activity in the functional networks, correlations are reported between the patterns of morphology, i.e. the structural covariance network (SCN) (chapter 8). For the first time, it is shown that properties of $\mathrm{SCN}$ are related to neurocognitive and epilepsy characteristics. Stronger clustering within the SCN is found to be associated with (i) an increased number of focal to bilateral tonic-clonic seizures during lifetime, (ii) a younger age at onset of epilepsy and (iii) lower cognitive performance scores. This indicates that the correlations between interregional variations of cortical thickness reflect disease characteristics or responses to the disease in adult patients with focal seizure epilepsy. Unfortunately, individual SCNs cannot be extracted directly, but require a healthy group [19]. Previously, it was shown that a healthy control group of approximately 25 subjects is sufficient to achieve stable and reliable results [19]. Therefore, the applicability of this method in the sample of children with CAE $(\mathrm{N}=17)$ and healthy controls $(\mathrm{N}=15)$ is limited. Previously, Curwood et al. already showed that the group-level SCN is altered in CAE [20]. However, since a group-level SCN was used, no further statistical analysis of neurocognitive and epilepsy characterises could be performed. Therefore, future studies with a larger cohort are needed to investigate whether the individual SCN analysis could be useful tool in CAE.

\section{Implications and directions future research}

Altogether, local myelin abnormalities, altered patterns of myelination, altered functional and myelin-weighted structural networks were observed in children with CAE. These combined findings in CAE might be part of the same underlying mechanisms. For example, altered myelin content could not only have a bearing on the structural network, but might also be related to the functional network. Electrical signals in the brain (i.e. action potentials) typically occur after a summation of multiple precisely timed signals (i.e. excitatory postsynaptic potentials). Alterations of the myelin content could interfere with these complex timing patterns, potentially causing different activation patterns in the brain.

Adult patients with a more severe form of focal seizure epilepsy (higher seizure frequency, decreased cognitive performance and a younger onset age) were associated with an increased clustering of the SCN (chapter 8). Interestingly, the results from chapter 7 as well as other DTI and fMRI studies show an opposite relation between cognitive performance and clustering [21], [22]. Previously, SCNs were found to only partly (35-40\%) reflect the axonal connections obtained using DTI [23]. Furthermore, since epilepsy has been associated with morphological changes [24], [25], the SCNs might provide unique knowledge on interregional cortical associations in epilepsy, and therefore provide 
information which is intrinsically different from fMRI and DTI. Segregation of the brain network into several clusters has been implied to be a containment mechanism for activity, such as epileptic seizures [26]. Therefore, the reported association could also be a compensatory mechanism, attempting to limit the effect of the seizures.

To conclude, this thesis outlines early examples of non-invasive myelin-specific imaging studies and the need to further investigate the relation of myelin content and epilepsy. For instance, larger studies with longitudinal designs are prompted to ascertain whether the myelin-water content worsens over time, whether altered myelin-water content predates the onset of absence epilepsy. 


\section{References}

[1] K. Moldovan et al., "Myelin water fraction changes in febrile seizures," Clin. Neurol. Neurosurg., vol. 175, pp. 61-67, 2018.

[2] H. S. Spader et al., "Advances in myelin imaging with potential clinical application to pediatric imaging," Neurosurg. Focus, vol. 34, no. 4, p. E9, Apr. 2013.

[3] E. Alonso-Ortiz, I. R. Levesque, and G. B. Pike, "MRI-based myelin water imaging: A technical review," Magn. Reson. Med., vol. 73, no. 1, pp. 70-81, Jan. 2015.

[4] K. P. Whittall and A. L. MacKay, "Quantitative interpretation of NMR relaxation data," J. Magn. Reson., vol. 84, no. 1, pp. 134-152, Aug. 1989.

[5] T. Prasloski et al., "Rapid whole cerebrum myelin water imaging using a 3D GRASE sequence," Neuroimage, vol. 63, pp. 533-539, Oct. 2012.

[6] S. Ocklenburg et al., "Myelin Water Fraction Imaging Reveals Hemispheric Asymmetries in Human White Matter That Are Associated with Genetic Variation in PLP1," Mol. Neurobiol., vol. 56, no. 6, pp. 3999-4012, 2019.

[7] T. D. Faizy et al., "Age-Related Measurements of the Myelin Water Fraction derived from 3D multi-echo GRASE reflect Myelin Content of the Cerebral White Matter," Sci. Rep., vol. 8, no. 1, p. 14991, 2018.

[8] N. Uddin, T. D. Figley, and C. R. Figley, "Effect of echo time and T2-weighting on GRASE-based T1w/T2w ratio measurements at 3T," Magn. Reson. Imaging, vol. 51, pp. 35-43, 2018.

[9] A. Petrovic, E. Scheurer, and R. Stollberger, "Closed-form solution for T2 mapping with nonideal refocusing of slice selective CPMG sequences," Magn. Reson. Med., vol. 73, no. 2, pp. 818-827, Feb. 2015.

[10] R. M. Lebel and A. H. Wilman, "Transverse relaxometry with stimulated echo compensation," Magn. Reson. Med., vol. 64, no. 4, pp. 1005-1014, Jun. 2010.

[11] A. Akhondi-Asl, O. Afacan, M. Balasubramanian, R. V. Mulkern, and S. K. Warfield, "Fast myelin water fraction estimation using 2D multislice CPMG," Magn. Reson. Med., vol. 76, no. 4, pp. 13011313, Oct. 2016.

[12] I. M. Vavasour, C. M. Clark, D. K. B. Li, and A. L. MacKay, "Reproducibility and reliability of MR measurements in white matter: Clinical implications," Neuroimage, vol. 32, pp. 637-642, Aug. 2006.

[13] W. S. Vargas et al., "Measuring longitudinal myelin water fraction in new multiple sclerosis lesions," Neurolmage Clin., vol. 9, pp. 369-375, 2015.

[14] C. Laule et al., "Water content and myelin water fraction in multiple sclerosis," J. Neurol., vol. 251, pp. 284-293, Mar. 2004.

[15] H. Chahboune, A. M. Mishra, M. N. Desalvo, L. H. Staib, M. Purcaro, and D. Scheinost, "DTI abnormalities in anterior corpus callosum of rats with spike-wave epilepsy," Neuroimage, vol. 47, pp. 459-466, 2009.

[16] R. Dietrich et al., "MR Evaluation of Early Myelination Patterns in Normal and Developmentally Delayed Infants than white," Am. J. Roentgenol., vol. 150, no. 4, pp. 889-896, 1988.

[17] S. Pajevic, P. J. Basser, and R. D. Fields, "Role of myelin plasticity in oscillations and synchrony of neuronal activity," Neuroscience, vol. 276, pp. 135-147, 2014.

[18] M. P. Van Den Heuvel, C. J. Stam, S. Kahn, and H. E. H. Pol, "Efficiency of Functional Brain Networks and Intellectual Performance," J. Neurosci., vol. 29, no. 23, pp. 7619-7624, 2009.

[19] M. Saggar et al., "Estimating individual contribution from group-based structural correlation networks.," Neuroimage, vol. 120, pp. 274-284, Oct. 2015. 
[20] E. K. Curwood, M. Pedersen, P. W. Carney, A. T. Berg, D. F. Abbott, and G. D. Jackson, "Abnormal cortical thickness connectivity persists in childhood absence epilepsy.," Ann. Clin. Transl. Neurol., vol. 2, no. 5, pp. 456-64, 2015.

[21] M. J. Vaessen et al., "White matter network abnormalities are associated with cognitive decline in chronic epilepsy," Cereb. Cortex, vol. 22, no. 9, pp. 2139-2147, 2012.

[22] M. C. G. Vlooswijk et al., "Loss of network efficiency associated with cognitive decline in chronic epilepsy," Neurology, vol. 77, no. 10, pp. 938-944, Sep. 2011.

[23] G. Gong, Y. He, Z. J. Chen, and A. C. Evans, "Convergence and divergence of thickness correlations with diffusion connections across the human cerebral cortex," Neuroimage, vol. 59, pp. 1239-1248, Jan. 2012.

[24] G. M. Overvliet et al., "Early onset of cortical thinning in children with rolandic epilepsy," NeuroImage Clin., vol. 2, pp. 434-439, 2013.

[25] B. C. Bernhardt et al., "Preferential susceptibility of limbic cortices to microstructural damage in temporal lobe epilepsy: A quantitative T1 mapping study," Neuroimage, vol. 182, pp. 294-303, 2017.

[26] L. Douw et al., "Dissociated multimodal hubs and seizures in temporal lobe epilepsy," Ann. Clin. Transl. Neurol., vol. 2, no. 4, pp. 338-352, Apr. 2015. 

Addendum 
Summary 
The aim of this thesis is to gain more insights into the relevance of myelin and cerebral networks as an underlying neurobiological substrate of epilepsy. For this, dedicated quantitative Magnetic resonance imaging (MRI) techniques were applied to various types of epilepsy and the techniques were advanced with a focus on image acquisition and analysis for the myelin content and cerebral networks.

Epilepsy is one of the most common serious neurological disorders, with a world-wide prevalence of about 6 to 9 per 1000 persons. It is most commonly known for its recurrent epileptic seizures, which are defined as a temporary occurrence of symptoms due to abnormal, excessive or synchronous neuronal activity in the brain. Besides the epileptic seizures, epilepsy is often accompanied by neurocognitive comorbidities. Since the neuronal cell bodies that discharge synchronously during an epileptic seizure reside mainly in the gray matter of the brain, epilepsy was traditionally thought to be mainly a disorder of the gray matter. However, recent research has shown that epilepsy affects the complex interactions of different brain regions connected by via the fiber bundles of the white matter. Therefore, epilepsy is currently regarded as a disorder of the brain network, in which the white matter also plays an important role.

MRI is a powerful tool to non-invasively visualize the anatomy of the brain. Anatomical MR images have been extensively applied to study local brain abnormalities in patients with epilepsy, potentially aiding diagnosis or eventual surgery planning. However, the epileptic brain often has other, more subtle abnormalities which are not visible at radiological inspection of anatomical images obtained by MRI. Therefore, advanced MRI techniques aimed at studying cerebral networks including the white matter are necessary to gain additional insights into epilepsy.

Chapter 1 provides a general introduction on epilepsy, which was traditionally thought to be a disorder of the gray matter, since the neuronal cell bodies that discharge synchronously during an epileptic seizure reside mainly in the gray matter (e.g. the cortex) of the brain. However, recent research has shown that the pathophysiology of epilepsy is not limited to the gray matter, but rather affects the complex interactions of different brain regions connected through axon bundles in the white matter. Thereafter, the advanced MRI techniques used to study epilepsy are introduced, covering; multi-echo T2 relaxometry to estimate the myelin-water fraction (MWF) (i.e. myelin content), as well as diffusion, functional and anatomic MRI to study various types of cerebral networks.

In chapter 2 the involvement of the myelin sheath in epilepsy is studied by reviewing the current literature. Abnormal myelination or demyelination is related to epilepsy in preclinical animal models as well as in human tissue. However, the currently available literature does not provide a complete view into the nature of myelin abnormalities in epilepsy. Therefore, future myelin imaging studies are prompted to provide new avenues for understanding the underling mechanisms in epilepsy, seizures and the interaction with the vulnerable and maturing brain during childhood. 
In chapter 3 an alternative analysis method to quantify the myelin content is proposed. We have applied the Orthogonal Matching Pursuit (OMP) to estimate the myelin content, and compared it to the most commonly applied algorithm, the Non-Negative Least Squares (NNLS) approach. Using numerical simulations and an in vitro phantom model it was demonstrated that the OMP is a more accurate method to estimate the MWF compared to the NNLS. Furthermore, in vivo results show similar findings between the two analysis methods as the simulations and phantom model, proving that OMP can be a preferred alternative to conventional methods for its accuracy and low computational complexity.

For MWF imaging to become more clinically feasible in patients with a variety of disorders, a rapid whole brain coverage with reproducible results is essential. Therefore, in chapter 4, the reproducibility of an accelerated MWF imaging technique was assessed. It was shown that the accelerated images are in excellent agreement with the reference method for MWF imaging. Furthermore, the accelerated MWF estimation with a scan time of 7:30 min was found to be highly reproducible.

The next three chapters set out to explore the involvement of myelin and structural/functional cerebral networks in a sample of children with childhood absence epilepsy (CAE) and healthy age-sex matched controls. CAE is an epilepsy syndrome that is characterized by seizures of brief losses of awareness occurring multiple times per day, in otherwise normally developing school-aged children.

In chapter 5 the myelin content was estimated using the new acquisition method. This chapter, for the first time, showed a lower myelin-water content in the frontal lobe of children with CAE compared to healthy peers, probably implicating an altered neurodevelopmental aspect in CAE.

Diffusion MRI enables the characterization of the structural brain network in terms of white matter tracts that interconnect distributed gray matter regions throughout the entire brain. In chapter 6 we investigated the development of myelin-water content of white tracts in healthy controls with ages ranging from 7 up to 32 years in a cross-sectional study. The myelin content was found to increase steadily throughout the whole brain from childhood into adulthood. Furthermore, the difference in myelin content between earlier and later myelinated tracts was lower in children with CAE, indicating that CAE is associated with widespread neurodevelopmental myelin differences.

Besides the macroscopic and microstructural components, cerebral regions are also known to show distinct temporal activity patterns in rest and during specific tasks using functional MRI (fMRI). The topological organization of the complex structural and functional interactions throughout the distributed regions of the brain can be described as a network. Graph theoretical analysis can then be used to calculate certain measures that characterize the brain network. In chapter 7, disruptions of the functional network in terms of weaker clustering and longer path lengths were observed in children with CAE compared to healthy controls. Moreover, longer path lengths were related to a worse seizure control and a longer duration of CAE. 
In chapter 8 the concept of the so-called structural covariance network (SCN) was applied to assess the relation between brain measurements and the severity of epilepsy in a sample of adult patients with epilepsy with focal seizures. The SCN is based on the correlations of morphological measures (e.g. thickness of the cortex) at various brain regions between subjects. It relies on the assumption that axonally connected regions have, to some extent, similar inter-subject variations and spatially distributed patterns of morphology. The clustering of the SCN was found to be associated with the severity of epilepsy in terms of more seizures, younger age at onset, and lower cognitive performance.

Chapter 9 provides a general discussion, here the findings of this thesis are discussed and integrated in a broader context and it provides directions for future research. 
Samenvatting 
Het doel van dit proefschrift is om meer inzicht te krijgen in de rol van myeline en hersennetwerken in epilepsie en de bijbehorende cognitieve bijwerkingen. Hiervoor is gebruik gemaakt van speciale kwantitatieve magnetic resonance imaging (MRI) beeldtechnieken, gericht op de myeline-inhoud en cerebrale netwerken.

Epilepsie is een van de meest voorkomende ernstige neurologische aandoeningen, wereldwijd hebben ongeveer 6 tot 9 per 1000 personen epilepsie. Deze aandoening is vooral bekend om de terugkerende epileptische aanvallen, die worden gedefinieerd als een tijdelijk optreden van symptomen als gevolg van abnormale, overmatige of synchrone neuronale activiteit in de hersenen. Naast de epileptische aanvallen gaat epilepsie vaak gepaard met cognitieve bijwerkingen. De neuronale cellen die tijdens een epileptische aanval overmatige en synchrone activiteit bevinden zich voornamelijk in de grijze stof. Daarom werd lange tijd gedacht dat epilepsie een ziekte van de grijze stof was. Echter, recenter onderzoek heeft aangetoond dat voor epilepsie de interacties tussen de door witte stof vezels verbonden hersengebieden belangrijk zijn. Daarom wordt epilepsie tegenwoordig gezien als een ziekte waarbij het netwerk van de hersenen is gewijzigd, en mogelijk zelfs aangetast, waarvoor de witte stof een belangrijke rol heeft.

MRI is een krachtige techniek waarmee de structuur van de hersenen in beeld kan worden gebracht op niet-invasieve wijze. Anatomische MRI-afbeeldingen worden uitgebreid gebruikt om hersenafwijkingen bij patiënten met epilepsie op te sporen, hetgeen kan mogelijk helpen bij de diagnose of bij de voorbereiding van een eventuele hersenoperatie. Daarnaast heeft het epileptische brein vaak andere, subtielere afwijkingen die niet zichtbaar zijn bij de radiologische inspectie van anatomische beelden. Daarom zijn geavanceerde MRI-technieken nodig om nieuwe inzichten te verkrijgen in epilepsie.

Hoofdstuk 1 geeft een algemene inleiding over epilepsie. Epilepsie werd lange tijd beschouwd als een aandoening van louter de grijze stof, omdat de neuronale cellen die synchroon ontladen tijdens een epileptische aanval zich voornamelijk in de grijze stof (de cortex) van de hersenen bevinden. Recent onderzoek heeft echter aangetoond dat de epilepsie zich niet beperkt tot de grijze massa, maar dat het door middel van complexe interacties van verschillende hersengebieden verbonden door axonbundels in de witte stof beïnloedt. Vervolgens worden de geavanceerde MRI-technieken geïntroduceerd die zijn gebruikt om epilepsie in dit proefschrift te bestuderen, waaronder; multi-echo T2 relaxometrie om de myeline-water fractie (MWF) te bepalen (d.w.z. myeline-gehalte), evenals diffusie, functionele en anatomische MRI om de cerebrale netwerken te bestuderen.

Hoofdstuk 2 bestaat uit een overzicht van de bestaande literatuur over de relatie tussen de myelineschede en epilepsie. Abnormale myelinisatie of demyelinisatie is gerelateerd aan epilepsie in preklinische onderzoek (diermodellen) en in menselijk weefsel. Echter, de beschikbare literatuur geeft een onvolledig beeld van de aard van myeline-afwijkingen bij epilepsie, en daarom zijn onderzoeken met myeline-beeldvormingstechnieken nodig om meer inzicht te krijgen in epilepsie en epileptische aanvallen. 
Hoofdstuk 3 gaat over de kwantificatie van het myeline-gehalte in de hersenen. Heir wordt een nieuwe data analysemethode voorgesteld om het myeline-gehalte te kwantificeren. De Orthogonal Matching Pursuit (OMP) is toegepast om het myeline-gehalte te bepalen, en de OMP is vergeleken met het meest toegepaste algoritme, de Non-Negative Least Squares (NNLS) methode. Met behulp van numerieke simulaties en een in vitro fantoommodel is aangetoond dat de OMP een meer accurate methode is voor de MWF-bepaling dan de NNLS. Bovendien laten de resultaten van metingen bij proefpersonen overeenstemmende bevindingen zien vergeleken met de simulaties en het fantoommodel. Hieruit blijkt dat OMP een goed alternatief kan zijn ten opzichte van de conventionele methoden.

Om MWF-beeldvorming beter klinisch toepasbaar te maken in verschillende patiënten en aandoeningen, zijn een snelle acquisitie van de gehele hersenen én reproduceerbare resultaten essentieel. Daarom is in hoofdstuk 4 de reproduceerbaarheid van een versnelde MWF-beeldvormingstechniek onderzocht. Hier wordt aangetoond dat de versnelde techniek uitstekend overeenkomt met de referentiemethode voor MWF-beeldvorming. Bovendien blijkt de versnelde MWF-bepaling, met een scantijd van 7:30 min, zeer goed reproduceerbaar te zijn.

De volgende drie hoofdstukken hebben betrekking op myeline en structurele/functionele cerebrale netwerken bij kinderen met absence epilepsie (AE) en gezonde controle kinderen. AE is een epilepsiesyndroom dat wordt gekenmerkt door aanvallen met kort bewustzijnsverlies die verschillende keren per dag optreden.

In hoofdstuk 5 is de MWF geschat met behulp van de nieuwe acquisitiemethode. In dit hoofdstuk is voor het eerst een lager myeline gehalte gevonden in de frontale kwab bij kinderen met AE.

Met behulp van diffusie-MRI kan het structurele hersennetwerk in beeld worden gebracht. Dit netwerk bestaat uit vele verbindingen (witte stof) die de neurale cellen (grijze stof) verbinden. In hoofdstuk 6 hebben we de rijping van het myeline gehalte van verschillende witte stof verbindingen onderzocht. Het myeline-gehalte blijkt gestaag toe te nemen vanaf de kindertijd tot volwassenheid. Bovendien waren er bij kinderen met $\mathrm{AE}$ verschillen in de myeline gehaltes van verbindingen die vroeg en laat ontwikkelen. Dit geeft aan dat AE naast lokale afwijkingen, ook wordt gekarakteriseerd door wijdverspreide myelineverschillen.

Voorgaande resultaten beperken zich tot de structurele componenten van het brein, echter is het daarnaast ook bekend dat hersengebieden bepaalde activiteitspatronen vertonen in rust en tijdens specifieke taken. Met behulp van functionele MRI (fMRI) kan de organisatie van de complexe functionele interacties in de verschillende hersengebieden worden beschreven als een netwerk. Vervolgens kunnen relevante eigenschappen van het netwerk bepaald worden met behulp van netwerkanalyses. In hoofdstuk 7 zijn verstoringen van het functionele netwerk waargenomen bij kinderen met $\mathrm{AE}$ in vergelijking met gezonde controles. Deze verstoringen uitten zich in een zwakkere clustering en langere paden van het 
functionele netwerk. De langere paden in het netwerk blijken verder gerelateerd aan een slechtere controle van de aanvallen en een langere duur van AE.

In hoofdstuk 8 wordt het zogenaamde Structural Covariance Network (SCN) gebruikt om de relatie tussen hersenstructuur en de ernst van epilepsie te beoordelen in volwassen patiënten met focale epilepsie. De SCN is gebaseerd op de correlaties tussen proefpersonen van morfologische metingen (bijv. de dikte van de cortex) in verschillende hersengebieden. Deze methode verondersteld dat verbonden regio's tot op een zekere hoogte vergelijkbare morfologische patronen hebben. De clustering van de SCN blijkt geassocieerd te zijn met hogere aanvalsfrequentie, lagere cognitieve prestaties en het krijgen van epilepsie op een jongere leeftijd.

Hoofdstuk 9 geeft een algemene discussie, hier worden de belangrijkste bevindingen van dit proefschrift besproken en in een bredere context geplaatst. 
Valorization 


\section{Relevance}

Magnetic resonance imaging (MRI) is a valuable tool to non-invasively gain information on cerebral abnormalities in patients with various neurological disorders. Anatomical MR images can potentially aid in diagnosis and/or treatment of the disorder [1]. However, the brain often has other, more subtle abnormalities which are not directly visible at radiological inspection of anatomical images obtained by MRI. This thesis focussed on cerebral abnormalities in patients with epilepsy, by employing advanced MRI techniques.

Epilepsy is one of the most common serious neurological disorders. The most characteristic feature of epilepsy is recurrent epileptic seizures, which can cause a wide range of outward effects from uncontrolled movement of (part of the) body to temporary losses of awareness. Besides the epileptic seizures, epilepsy is often accompanied by neurocognitive comorbidities [2]. In rare and very severe cases epilepsy can result in death. Epilepsy has a world-wide prevalence of about 6 to 9 per 1000 persons [3]. More specific, in the Netherlands 51.678 people were estimated to have epilepsy in 2016 of which 268 died as a result of the disorder [4].

Since the neuronal cell bodies that discharge synchronously during an epileptic seizure reside mainly in the gray matter of the brain, epilepsy was traditionally thought to be mainly a disorder of the gray matter [5]. However, recent research has shown that the disruptions of brain tissue in epilepsy affect the complex interactions of different brain regions connected by via the fiber bundles of the white matter. Therefore, epilepsy is currently regarded as a brain network disorder, for which the white matter also plays an important role [6].

A possible underlying factor of a hyper-excitable state of the epileptic brain is the temporal summation of several synchronous subthreshold excitatory stimuli [7]. This summation could be a direct consequence of axons with poorly distributed conduction velocities that result in the synchronous firing of action potentials. The conduction velocity of an axon is mainly related to its diameter and the myelin sheath. Therefore, a direct relation might exist between epileptic seizure susceptibility and an abnormal myelin content.

\section{Main Findings}

The main clinical finding of this thesis is the involvement of the myelin sheath in patients with epilepsy. Numerous studies have already hinted towards myelin abnormalities in preclinical epilepsy models and patients with epilepsy (chapter 2). Furthermore, a disrupted myelin content and myelin development was observed in children with epilepsy (chapters 5 and 6). Besides the clinical findings, this thesis also provided technical advances. First, an alternative analysis method to quantify the myelin content is proposed. The presented method provides more measures of myelin content compared to a widely-used alternative (chapter 3). In chapter 4 of this thesis, we introduced an adapted myelin imaging acquisition that is less 
prone to head movement and has an acquisition time that is roughly twice as short compared to previous versions.

\section{Target group}

The new clinical insights of this thesis could potentially, in the long run, be beneficial to patients with epilepsy. New treatment regimens aimed towards the myelin sheath could have a positive effect on the disorder. However, it should be noted that from this thesis alone it cannot be concluded that abnormalities of the myelin sheath are a cause or consequence of epilepsy and/or epileptic seizures. Therefore, first, further research on the involvement of the myelin sheath in epilepsy is warranted.

The technical advances presented in this thesis can also benefit patients suffering from other myelin related neurological disorders, such as Alzheimer's disease [8], multiple sclerosis [9], Parkinson's disease [10], major depressive disorder [11] and schizophrenia [12]. The faster myelin acquisition method can reduce the total scanning time of future clinical studies, which is mainly beneficial for patients that cannot sustain long MRI scanning times, such as elderly patients, patients with movement disorders, young children and infants. Furthermore, the available MRI scanning time can be used more cost efficiently by hospitals.

The algorithm used to quantify the myelin content (chapter 3 ) is made publicly available as an open-source project on GitHub (https://github.com/GSDrenthen/Non-Negative-OMP). Therefore, other researches that are interested in the myelin content can easily implement the analysis.

\section{Products and activities}

Since myelin underlies several neurological and neuropsychiatric disorders, insights into the cerebral myelination could aid in the diagnostic and therapeutic strategies on an individual level. As such, the myelin content of an individual can be compared with measures from a large population to identify whether individual maps fall within the population distribution, or whether they constitute abnormal values. This approach of personalized medicine can be incorporated as a software application on a clinical scanner. Like this, undergoing a brain MRI exam, a myelin map can be acquired, and subsequently relevant deviations in myelination will be visualized. This could ultimately be used to determine the optimal therapeutic strategy and/or disease progression precisely tailored per individual. 


\section{Innovation and future directions}

The research in this thesis indicates that epilepsy is related to abnormalities of the myelin sheath. However, the current evidence from advanced MRI methods is relatively scarce. Therefore, myelin-specific imaging methods should be employed in other (preferably larger) epilepsy studies. Furthermore, to explore whether myelin abnormalities are a cause or consequence of epilepsy longitudinal studies are required. Like this, it can for example be established whether the myelin abnormalities worsen over time. While this does not provide concluding causal evidence, it does provide new insights into the myelin-epilepsy relation.

Furthermore, this thesis shows that the acquisition of myelin-specific content can be accelerated by using multi-slice 2D scanning methods. Recently, under-sampling methods such as compressed sensing are used to decrease scanning times [13]. Therefore, further advances to reduce the acquisition time of the $2 \mathrm{D}$ pulse sequence can still be made.

Moreover, in the future, the acquisition of myelin-specific scans could be omitted entirely. Anatomical and diffusion MRI techniques relate to the myelin content and are already acquired in most clinical research studies. Although these techniques are not suitable for absolute quantification of myelin, combined they might exhibit an underlying (complex and non-linear) relation to the myelin content. Therefore, machine learning approaches can be employed to extract myelin-specific information from anatomical and diffusion MRI data. 


\section{References}

[1] F. Cendes, W. H. Theodore, B. H. Brinkmann, V. Sulc, and G. D. Cascino, "Neuroimaging of epilepsy," Handb Clin Neurol., vol. 136, pp. 985-1014, 2016.

[2] J. J. Lin, M. Mula, and B. P. Hermann, “Uncovering the Lifespan Neurobehavioral Comorbidities of Epilepsy," Lancet, vol. 380, no. 9848, pp. 1180-1192, 2013.

[3] K. M. Fiest et al., "Prevalence and incidence of epilepsy A systematic review and meta-analysis of international studies," Neurology, vol. 88, pp. 296-303, 2017.

[4] GBD 2016 Epilepsy Collaborators, "Global, regional, and national burden of epilepsy, 1990-2016: a systematic analysis for the Global Burden of Disease Study 2016," Lancet Neurol., vol. 18, pp. 357375, 2019.

[5] E. H. Reynolds, "Jackson, Todd, and the Concept of 'Discharge' in Epilepsy," vol. 48, no. 11, pp. 2016-2022, 2016.

[6] W. M. Otte, P. Van Eijsden, J. W. Sander, J. S. Duncan, R. M. Dijkhuizen, and K. P. J. Braun, “A meta-analysis of white matter changes in temporal lobe epilepsy as studied with diffusion tensor imaging," Epilepsia, vol. 53, no. 4, pp. 659-667, 2012.

[7] E. B. Bromfield, J. E. Cavazos, and J. I. Sirven, Eds., An Introduction to Epilepsy. West Hartford (CT): American Epilepsy Society, 2006.

[8] E. Kavroulakis et al., "Myelin content changes in probable Alzheimer's disease and mild cognitive impairment: Associations with age and severity of neuropsychiatric impairment," J. Magn. Reson. Imaging, vol. 47, no. 5, pp. 1359-1372, 2018.

[9] S. Kolind et al., "Clinical Brain and cord myelin water imaging: a progressive multiple sclerosis biomarker," Neurolmage Clin., vol. 9, pp. 574-580, 2015.

[10] D. C. Dean III et al., "Alterations of Myelin Content in Parkinson's Disease : A Cross-Sectional Neuroimaging Study," PLoS One, vol. 11, no. 10, p. e0163774, 2016.

[11] M. D. Sacchet and I. H. Gotlib, "Myelination of the brain in Major Depressive Disorder: An in vivo quantitative magnetic resonance imaging study," Sci. Rep., vol. 7, p. 2200, 2017.

[12] D. J. M. Lang et al., "Clinical 48 echo T2 myelin imaging of white matter in first-episode schizophrenia: Evidence for aberrant myelination," NeuroImage Clin., vol. 6, pp. 408-414, 2014.

[13] A. Cristobal-Huerta, D. H. J. Poot, M. W. Vogel, G. P. Krestin, and J. A. Hernandez-Tamames, “Compressed Sensing 3D-GRASE for faster High-Resolution MRI," Magn. Reson. Med., vol. 82, no. 3, pp. 984-999, 2019. 
Dankwoord 
Hoewel mijn naam groot op de voorkant van dit proefschrift staat, zijn er natuurlijk veel meer mensen die belangrijk zijn geweest bij de realisatie van dit proefschrift. Deze bijdragen waren lang niet altijd alleen inhoudelijk. In dit laatste hoofdstuk van mijn proefschrift wil ik graag iedereen bedanken voor hun bijdrage.

Ten eerste wil ik graag mijn promotieteam bedanken voor de top begeleiding van de afgelopen vier jaar. Walter Backes, Bert Aldenkamp en Jaap Jansen, ik heb veel geleerd, zowel inhoudelijk als persoonlijk, en hiervoor wil ik jullie enorm bedanken!

Jaap, jij was mijn dagelijkse begeleider en met jou heb ik tijdens mijn promotie het meest direct samengewerkt. Jouw deur stond altijd voor mij open, en je kritische en pragmatische instelling waren zeer waardevol voor mij. Terwijl ik zelf vaak geneigd ben om meer tijd te stoppen in het uitproberen van nieuwe analyse methodes, heb ik van jou geleerd om resultaatgerichter te werken.

Walter, ook bij jou kon ik altijd aankloppen als ik ergens mee zat. Zo'n toegankelijke eerste promotor, dat is als promovendus een enorme luxe! Tijdens onze structurele afspraken kwam jij vaak met nieuwe inzichten en mogelijkheden, wat het afgeleverde werk sterk heeft verbeterd. Verder hoefde ik op terugkoppeling van mijn werk nooit lang te wachten, van 's ochtends vroeg tot zelfs in de weekenden, jij reageerde altijd heel snel.

Bert, tijdens mijn Electrical Engineering studie ben ik bij jouw inauguratie lezing geïnspireerd geraakt om de laatste jaren van mijn studie te focussen op hersenonderzoek. Mijn afstudeerproject onder jouw begeleiding beviel zo goed dat ik gekozen heb voor dit promotietraject. Tijdens de afgelopen vier jaar was jouw klinisch inzicht en kennis altijd enorm waardevol.

Vervolgens wil ik graag de leden van de beoordelingscommissie bedanken, Prof. dr. Felix Mottaghy, Prof. dr. Kees Braun, Prof. dr. David Linden en Prof. dr. ir. Peter de With, voor de tijd die zij hebben genomen om mijn proefschrift te beoordelen.

Een wetenschappelijk artikel publiceer je zelden alleen, en dat gaat met name op in dit vakgebied, met raakvlakken vanuit de kliniek en de technologie. Daarom wil ik ook graag alle coauteurs bedanken voor hun waardevolle inbreng.

Eric, als mijn klinische-tweeling voor de LEES studie heb ik veel kunnen leren van jou. Jij hebt enorm veel werk geleverd aan het studieprotocol en de inclusie, waarvoor enorm bedankt! We hebben samen veel gezellige uren gemaakt bij de scanner, maar ook vaak kritisch onderling overlegd. Dit heeft uiteindelijk mooie resultaten opgeleverd. Bedankt voor de hele fijne samenwerking!

Ook wil ik graag de coauteurs Jeroen Vermeulen, Sylvia Klinkenberg, Giel Op 't Veld, Mariette Debeij-van Hall, Jos Hendriksen, Floor Fasen, Rob Rouhl, Marielle Vlooswijk, Marian Majoie en Paul Hofman bedanken voor hun bijdrage aan de wetenschappelijke publicaties. 
Daarnaast wil ik de deelnemende kinderen en hun ouders van de LEES studie en de andere vrijwilligers bedanken, zonder jullie inbreng was dit proefschrift er niet geweest. Ook wil ik graag de MRI laboranten in Kempenhaeghe, Remco Berting en Henri Saes, bedanken voor de vele uren die we gemaakt hebben achter de scanner. Bovendien, Remco, jouw ervaring met het scannen van kinderen heeft veel geholpen in de opzet van scanprotocol, bedankt!

De data verkrijgen is de eerste stap, maar vervolgens moet het worden geanalyseerd. Gelukkig was dit bij de afdeling Radiologie in Maastricht geen probleem vanwege de enorme rekenkracht die de promovendi tot hun beschikking krijgen. Hiervoor wil ik graag Marc, Jos en Jan bedanken!

De prettige werksfeer in Maastricht heeft ook een belangrijke bijdrage geleverd aan het afronden van mijn proefschrift, en dat heb ik met name te danken aan mijn gezellige collega's! Harm, Tamar, May, Laura, Lisanne, Joost, Marieke, Merel, Jennifer, Harmen, Floor en Paulien, bedankt voor de gezelligheid in Maastricht! Ook wil ik graag de overige collega's van de afdeling Radiologie in Maastricht bedanken.

Vervolgens wil ik ook graag de collega promovendi in Eindhoven bedanken voor de gezelligheid, Stephan, Antoine, Debby, Maarten en Elles. Daarnaast Sveta, bedankt voor de hulp en begeleiding bij het opzetten van de LEES studie!

Ook wil ik graag mijn vrienden van EE generatie 2007 bedanken! Onze borrels, diners, pokeren spellenavonden, wie is de mol weekenden, en andere activiteiten zorgden voor (meer dan) voldoende ontspanning tijdens de afgelopen vier jaren. Bedankt!

Naast mijn collega's en vrienden ben ik ook veel dank verschuldigd aan mijn familie en schoonfamilie. Pap en Mam, zonder jullie was ik natuurlijk nooit zover gekomen. Bedankt voor jullie onvoorwaardelijke steun en onuitputbaar vertrouwen in mij.

Zusje! Hoewel we nogal verschillen qua persoonlijkheid (ik de nerd en jij de manager) ben ik blij dat jij mijn zus bent. Bedankt! Natuurlijk kunnen Arthur en de kleine Lynn ook niet ontbreken. Arthur, jij toonde altijd enorm veel interesse in mijn werkzaamheden. En mijn nichtje Lynn, hoewel het nog even zal duren voordat je dit kunt lezen, bedankt voor alle ontspannende uren!

Peter en Constance, ik heb mij de afgelopen jaren altijd erg welkom gevoeld in Mheer. Bedankt voor alle gezellige en verrijkende uitjes die we hebben gemaakt, en voor alle heerlijke maaltijden.

Als laatste wil ik degene bedanken die voor mij het allerbelangrijkste is. Samen hebben wij de afgelopen vier jaar in hetzelfde schuitje gezeten, en wij hebben ons door de moeilijke momenten heen kunnen slaan. Jij zorgt ervoor dat ik het beste uit mezelf kan halen. Lieve Lieneke, bedankt. Ik hoop samen met jou nog veel nieuwe uitdagingen aan te gaan! 

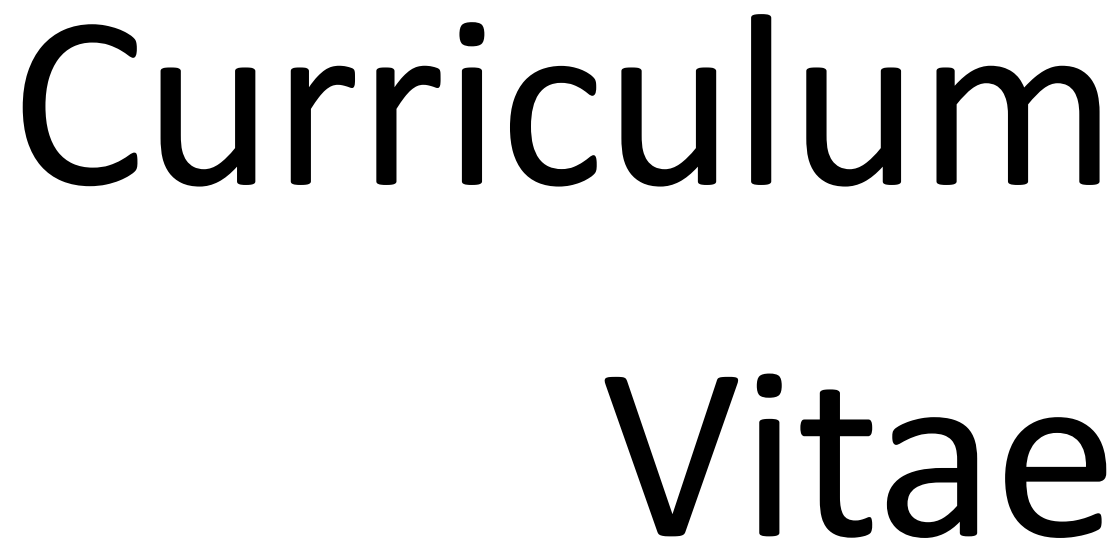


\section{Curriculum Vitae}

Gerhard Sebastiaan Drenthen was born on January $27^{\text {th }} 1989$ in Almelo, the Netherlands. After graduating secondary school in 2007 at the CSG Het Noordik in Almelo, he started his academic education at the Eindhoven University of Technology, where he obtained his Bachelor of Science degree (BSc) in August 2012 and his Master of Science degree (MSc) in Electrical Engineering in July 2015. During the Master program, he did an internship at the Industrial Automation Business division of Omron in Kusatsu, Japan (July 2013 - October 2013). Furthermore, he conducted his graduation project, entitled "Study of alterations in the brain for two neurological disorders, based on Magnetic Resonance Spectroscopy (MRS) and Imaging (MRI)", in collaboration with the department of Radiology of Maastricht University under supervision of Prof. dr. Albert P. Aldenkamp, dr. ir. Sveta Zinger and dr. Jacobus F.A. Jansen. In October 2015 he started his PhD project at the department of Radiology and the School of Mental Health \& Neuroscience of Maastricht University Medical Center. The research, as described in the present thesis, was performed under the supervision of promotores Prof. dr. ir. Walter H. Backes and Prof. dr. Albert P. Aldenkamp, and copromotor dr. Jacobus F.A. Jansen. During his PhD, Gerhard received accolades for his work including invited presentations, student stipends and best poster awards.

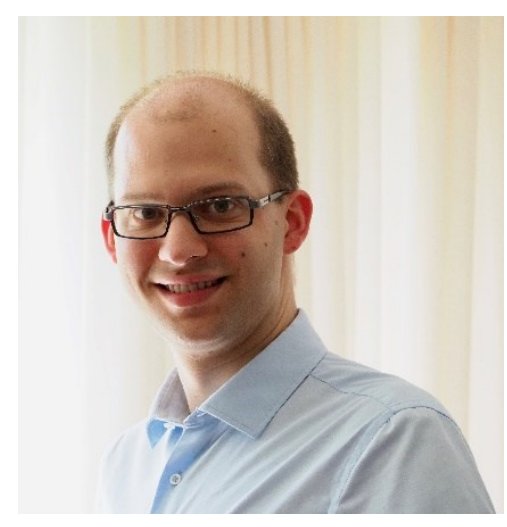




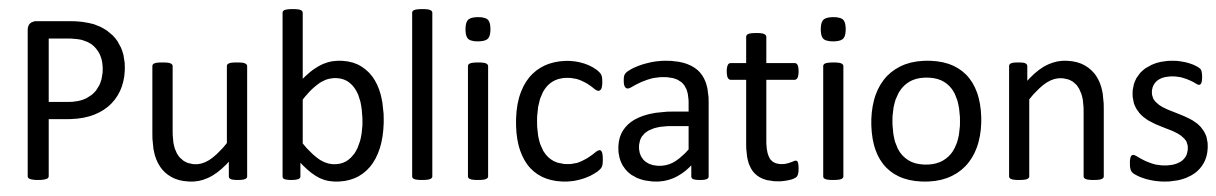




\section{Journal Contributions}

\section{This thesis}

Drenthen GS*, Fonseca Wald ELA*, Backes WH, Debeij-Van Hall MHJA, Hendriksen JGM, Aldenkamp AP, Vermeulen RJ, Klinkenberg S, Jansen JFA. Lower myelin-water content of the frontal lobe in childhood absence epilepsy. Epilepsia 2019, 60(8):1689-1696.

Drenthen GS, Backes WH, Aldenkamp AP, Jansen JFA. Applicability and reproducibility of 2D multi-slice GRASE myelin water fraction with varying acquisition acceleration. Neuroimage 2019, 195:333-339.

Drenthen GS, Backes WH, Aldenkamp AP, Op 't Veld GJ, Jansen JFA. A new analysis approach for $\mathrm{T}_{2}$ relaxometry myelin water quantification: Orthogonal Matching Pursuit. Magn Reson Med 2019, 81(5):3292-3303.

Drenthen GS, Backes WH, Rouhl RPW, Vlooswijk MCG, Majoie MHJM, Hofman PAM, Aldenkamp AP, Jansen JFA. Structural covariance networks relate to the severity of epilepsy with focal-onset seizures. Neuroimage Clin 2018, 20:861-867.

Drenthen GS*, Fasen $F^{*}$, Fonseca Wald ELA, Backes WH, Aldenkamp AP, Vermeulen RJ, Debeij-van Hall M, Hendriksen JGM, Klinkenberg S, Jansen JFA. Functional brain integrity is related to epilepsy severity in childhood absence epilepsy. Submitted.

Drenthen GS, Backes WH, Aldenkamp AP, Vermeulen RJ, Klinkenberg S, Jansen JFA. On the merits of non-invasive myelin imaging in epilepsy, a literature review. Submitted.

Drenthen GS, Backes WH, Fonseca Wald ELA, Aldenkamp AP, Vermeulen JR, Debeij-van Hall M, Klinkenberg S, Jansen JFA. Constructing an axonal-specific myelin developmental atlas and its application to childhood absence epilepsy. Submitted.

*Both authors contributed equally

\section{Other}

Drenthen GS, Barendse EM, Aldenkamp AP, van Veenendaal TM, Puts NA, Edden RA, Zinger S, Thoonen G, Hendriks MP, Kessels RP, Jansen JFA. Altered neurotransmitter metabolism in adolescents with high-functioning autism. Psychiatry Res Neuroimaging 2016, 256:44-49. 
Fonseca Wald ELA, Hendriksen J, Drenthen GS, Kuijk SMJ, Aldenkamp AP, Vles JSH, Vermeulen RJ, Debeij-van Hall M, Klinkenberg S. Towards a better understanding of cognitive deficits in absence epilepsy: a systematic review and meta-analysis. Neuropsychology Review. 2019, 29(4):421-449.

Wong SM, Backes WH, Drenthen GS, Zhang CE, Voorter PHM, Staals J, van Oostenbrugge RJ, Jansen JFA. Spectral Diffusion Analysis of Intravoxel Incoherent Motion MRI in Cerebral Small Vessel Disease. J Magn Reson Imaging. 2019, Epub ahead of print.

\section{Oral presentations}

Drenthen GS, Backes WH, Fonseca Wald ELA, Klinkenberg S, Vermeulen RJ, Hendriksen J, Debeij van Hall M, Aldenkamp AP, Jansen JFA. Characterizing healthy development of axonal-myelin Cross-sectional maturational differences in childhood absence epilepsy. $36^{\text {th }}$ Annual meeting of the ESMRMB 2019, Rotterdam. (Pitch)

Drenthen GS, Fonseca Wald ELA, Backes WH, Klinkenberg S, Debeij-van Hall M, Vermeulen RJ, Aldenkamp AP, Jansen JFA. T2 relaxometry reveals frontal demyelination in children with childhood absence epilepsy. $11^{\text {th }}$ Annual meeting of the ISMRM Benelux, 2019, Leiden. (Pitch)

Drenthen GS, Jansen JFA, Backes WH, Aldenkamp AP. Myelin-water quantification in children with early absence epilepsy. Junior Researchers day, Department of Radiology and Nuclear Medicine, 2019, Maastricht.

Drenthen GS, Jansen JFA, Backes WH, Aldenkamp AP. Myelin-water quantification in children with early absence epilepsy. Junior Researchers day, Department of Radiology and Nuclear Medicine, 2018, Maastricht.

Drenthen GS, Backes WH, Aldenkamp AP, Jansen JFA. Myelin-Water Quantification: Orthogonal Matching Pursuit versus Non-Negative Least Squares. $10^{\text {th }}$ Annual meeting of the ISMRM Benelux, 2018, Antwerp.

Drenthen GS, Jansen JFA, Backes WH, Aldenkamp AP. Myeline kwantificatie met behulp van MRI: vergelijking van verschillende analytische technieken. Wetenschapsdag (Scienceday) 2018, Department of Neurology, 2017, Maastricht.

Drenthen GS, Jansen JFA, Backes WH, Aldenkamp AP. Myelin imaging in children with early absence epilepsy. Junior Researchers day, Department of Radiology and Nuclear Medicine, 2017, Maastricht. 
Drenthen GS, Vlooswijk MCG, Majoie HJM, Hofman PAM, Aldenkamp AP, Backes WM, Jansen JFA. Individual measures of network efficiency in patients with epilepsy based on cortical thickness. $24^{\text {th }}$ Annual meeting of the ISMRM, May $11^{\text {th }}$ 2016, Singapore. Program number: 0779.

Drenthen GS, Jansen JFA, Backes WH, Aldenkamp AP. Longitudinal Early Epilepsy Study. Junior Researchers day, Department of Radiology and Nuclear Medicine, 2016, Maastricht.

Drenthen GS, Aldenkamp AP, Jansen JFA. Altered connectivity in epilepsy patients based on cortical thickness: A graph analysis. ${ }^{\text {st }}$ IEEE West-European Student and Young Professional Congress, 2015, Eindhoven. (Pitch)

\section{Poster presentations}

Drenthen GS, Backes WH, Fonseca Wald ELA, Klinkenberg S, Vermeulen RJ, Hendriksen J, Debeij van Hall M, Aldenkamp AP, Jansen JFA. Characterizing healthy development of axonal-myelin Cross-sectional maturational differences in childhood absence epilepsy. $36^{\text {th }}$ Annual meeting of the ESMRMB 2019, Rotterdam.

Drenthen GS, Backes WH, Fonseca Wald ELA, Klinkenberg S, Vermeulen RJ, Hendriksen J, Debeij van Hall M, Aldenkamp AP, Jansen JFA. Myelin quantification in children with childhood absence epilepsy. 11 $1^{\text {th }}$ Annual meeting of the ISMRM Benelux, 2019, Antwerp.

Drenthen GS, Backes WH, Fonseca Wald ELA, Klinkenberg S, Vermeulen RJ, Hendriksen J, Debeij van Hall M, Aldenkamp AP, Jansen JFA. Characterizing healthy development of axonal-myelin: Cross-sectional maturational differences in childhood absence epilepsy. $21^{\text {st }}$ Annual international clinical symposium Kempenhaeghe, 2019, Heeze.

Drenthen GS, Backes WH, Fonseca Wald ELA, Klinkenberg S, Vermeulen RJ, Hendriksen J, Debeij van Hall M, Aldenkamp AP, Jansen JFA. Myelin quantification in children with childhood absence epilepsy. MHeNS Research day, 2018, Maastricht.

Drenthen GS, Backes WH, Aldenkamp AP, Jansen JFA. Myelin-Water Quantification: Orthogonal Matching Pursuit versus Non-Negative Least Squares. 26 $6^{\text {th }}$ Annual meeting of the ISMRM, 2018, Paris.

Drenthen GS, Backes WH, Aldenkamp AP, Jansen JFA. Reproducibility of Myelin Water Fraction for GRASE sequences with a varying SENSE factor. $26^{\text {th }}$ Annual meeting of the ISMRM, 2018, Paris. 
Drenthen GS, Backes WH, Vlooswijk MCG, Majoie HJM, Hofman PAM, Aldenkamp AP, Jansen JFA. Altered structural correlation networks (SCN's) in cryptogenic localizationrelated epilepsy (CLRE) are associated with seizure frequency. 32 ${ }^{\text {nd }}$ International Epilepsy Congress, 2017, Barcelona.

Drenthen GS, Backes WH, Aldenkamp AP, Jansen JFA. Myelin quantification using Magnetic Resonance Imaging: comparison of different analytic techniques. $4^{\text {th }}$ IEEE SBE poster contest, 2017, Eindhoven.

Drenthen GS, Backes WH, Vlooswijk MCG, Majoie HJM, Hofman PAM, Aldenkamp AP, Jansen JFA. Altered structural correlation networks (SCN's) in cryptogenic localizationrelated epilepsy (CLRE) are associated with seizure frequency. 19 ${ }^{\text {th }}$ Annual international clinical symposium Kempenhaeghe, 2017, Heeze. *

Drenthen GS, Backes WH, Aldenkamp AP, Jansen JFA. Myelin Water Fraction estimation using a two-step exponential model - Phantom and in vivo results. $9^{\text {th }}$ Annual meeting of the ISMRM Benelux, 2017, Tilburg.

Drenthen GS, Backes WH, Aldenkamp AP, Jansen JFA. Myelin Water Fraction estimation using a two-step exponential model - Phantom and in vivo results. MHeNS Research day, 2016, Maastricht.

Drenthen GS, Aldenkamp AP, Jansen JFA. A Comparison of Graph Analysis and Maximum Spanning Tree Analysis in Epilepsy. $3^{\text {rd }}$ IEEE SBE poster contest, 2016, Eindhoven.

Drenthen GS, Vlooswijk MCG, Majoie HJM, Hofman PAM, Aldenkamp AP, Backes WH, Jansen JFA. Individual measures of network efficiency in patients with epilepsy, based on cortical thickness. $8^{\text {th }}$ Annual meeting of the ISMRM Benelux, 2016, Eindhoven.

Drenthen GS, Aldenkamp AP, Jansen JFA. Altered connectivity in epilepsy patients based on cortical thickness: A graph analysis. ${ }^{\text {st }}$ IEEE West-European Student and Young Professional Congress, 2015, Eindhoven. *

${ }^{*}$ Best poster award 
